\title{
New Potent Acetylcholinesterase Inhibitors in the
}

\section{Tetracyclic Triterpene Series}

Thibault Sauvaître, ${ }^{\dagger}$ Mireille Barlier, ${ }^{\dagger}$ Denyse Herlem, ${ }^{\dagger}$ Nohad Gresh ${ }^{\S}$, Angèle Chiaroni, ${ }^{\dagger}$ Daniel Guenard, ${ }^{*}{ }^{\dagger}$ and Catherine Guillou* ${ }^{\dagger}$

Institut de Chimie des Substances Naturelles, Bt 27, CNRS, Avenue de la Terrasse, 91198 Gifsur-Yvette, France, Laboratoire de Pharmacochimie Moléculaire et Cellulaire, CNRS FRE 2718, U648 INSERM, Université René Descartes (Paris V), 45 rue des Saints-Pères, 75006 Paris, France. 
Table of contents

Preparation of compound 5

Preparation of compound 7a

Preparation of compound $\mathbf{7 b}$

S6

Preparation of compound 7c

S7

Preparation of compound 7d

S8

Preparation of compound $\mathbf{7 e}$

S9

Preparation of compound $7 \mathbf{f}$

Preparation of compound $\mathbf{7 h}$

Preparation of compound 7i

Preparation of compound $\mathbf{8 b}$

Preparation of compound 8c

Preparation of compound $\mathbf{8 d}$

Preparation of compound $\mathbf{8 e}$

Preparation of compound $\mathbf{8 f}$

Preparation of compound $\mathbf{8 g}$

Preparation of compound $\mathbf{8 h}$

Preparation of compound $\mathbf{8 i}$

Preparation of compound $\mathbf{9 b}$

Preparation of compound 9c

Preparation of compound 9d

Preparation of compound 9e

Preparation of compound $9 f$

Preparation of compound $\mathbf{9 g}$

$\begin{array}{ll}\text { Preparation of compound } 9 \mathbf{h} & \text { S27 }\end{array}$

$\begin{array}{ll}\text { Preparation of compound 9i } & \text { S28 }\end{array}$

$\begin{array}{lr}\text { Preparation of compound } \mathbf{1 0} & \text { S29 }\end{array}$

$\begin{array}{lr}\text { Preparation of compound } \mathbf{1 1} & \text { S30 }\end{array}$

$\begin{array}{ll}\text { Preparation of compound } \mathbf{1 2} & \text { S31 }\end{array}$

$\begin{array}{ll}\text { Preparation of compound } \mathbf{1 3} & \text { S32 }\end{array}$

$\begin{array}{ll}\text { Preparation of compound } 14 & \text { S33 }\end{array}$

$\begin{array}{ll}\text { Preparation of compound } \mathbf{1 5} & \text { S34 }\end{array}$

$\begin{array}{lr}\text { Preparation of compound } \mathbf{1 6} & \text { S35 }\end{array}$

$\begin{array}{ll}\text { Preparation of compound } \mathbf{1 7} & \text { S36 }\end{array}$

$\begin{array}{ll}\text { Preparation of compound } 18 & \text { S37 }\end{array}$

$\begin{array}{lr}\text { Preparation of compound } \mathbf{1 9} & \text { S38 }\end{array}$

$\begin{array}{lr}\text { Preparation of compound 20a } & \text { S39 }\end{array}$

$\begin{array}{lr}\text { Preparation of compound 20b } & \text { S40 }\end{array}$

$\begin{array}{ll}\text { Preparation of compound 21a } & \text { S41 }\end{array}$

$\begin{array}{ll}\text { Preparation of compound 21b } & \text { S42 }\end{array}$

$\begin{array}{ll}\text { Preparation of compound 22a } & \text { S43 }\end{array}$

$\begin{array}{ll}\text { Preparation of compound 22b } & \text { S44 }\end{array}$

$\begin{array}{ll}\text { Preparation of compound 23a } & \text { S45 }\end{array}$

$\begin{array}{ll}\text { Preparation of compound 23b } & \text { S46 }\end{array}$

$\begin{array}{ll}\text { Preparation of compound 24a } & \text { S47 }\end{array}$

$\begin{array}{ll}\text { Preparation of compound 24b } & \text { S48 }\end{array}$

$\begin{array}{ll}\text { Preparation of compound 25a } & \text { S49 }\end{array}$

$\begin{array}{ll}\text { Preparation of compound 25b } & \text { S50 }\end{array}$

$\begin{array}{ll}\text { Preparation of compound 26a } & \text { S51 }\end{array}$ 
Preparation of compound 26b

Preparation of compound $\mathbf{2 7}$

Preparation of compound $\mathbf{2 8}$

S54

Preparation of compound $\mathbf{2 9}$

S55

Preparation of compound $\mathbf{3 0}$

S56

Preparation of compound $\mathbf{3 1}$

S57

Preparation of compound $\mathbf{3 2}$

S58

Preparation of compound $\mathbf{3 3}$

S59

Table of elemental analysis

Crystal data and structure refinement for compound $\mathbf{1}$

S62 
Detailed experimental procedures and compound characterization data for $\mathbf{5}, \mathbf{7 a - 7 i}, \mathbf{8 b}-\mathbf{8 i}, \mathbf{9 a}$ 9i, 10-19, 20a-26b, 27-33. The assignement of the ${ }^{1} \mathrm{H}$ and ${ }^{13} \mathrm{C}$ NMR spectra of the different compounds has been made by ${ }^{1} \mathrm{H}-{ }^{1} \mathrm{H},{ }^{1} \mathrm{H}-{ }^{13} \mathrm{C}$, COSY, HMBC, HMQC and by analogy with previously described compounds. The numerotation indicated on molecule is only mentioned for the NMR spectra interpretation. 


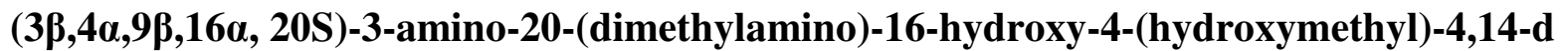
imethyl-9,19-cyclopregnan-11-one (5)

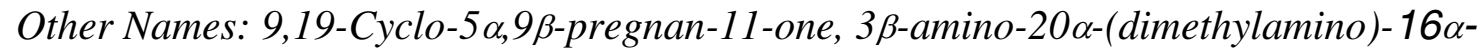

hydroxy-4a-(hydroxymethyl)-4,14-dimethyl; $11 \mathrm{H}, 19 \mathrm{H}$ -

Cyclopropa[9,10]cyclopenta[a]phenanthrene, 9,19-cyclopregnan-11-one deriv.; (+)-

Cycloxobuxidine F; Baleabuxidine F; Cycloxobuxidin F; Cycloxobuxidine F

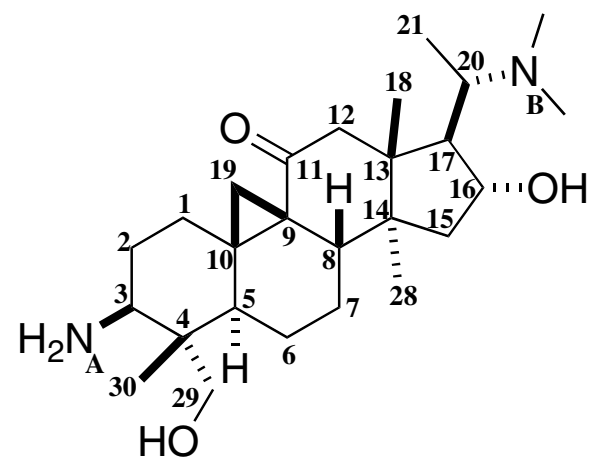

To a solution of $N$-3-isobutyrylcyclobuxidine-F $2(821 \mathrm{mg}, 1.63 \mathrm{mmol})$ in $30 \mathrm{~mL}$ of methanol were added $30 \mathrm{~mL}$ of an aqueous solution of sulfuric acid $(2 \mathrm{~N})$ at room temperature. After stirrring for 3 hours at $90{ }^{\circ} \mathrm{C}$, the reaction mixture was cool at room tempertature and evaporated under reduced pressure at $20{ }^{\circ} \mathrm{C}$. The resulting solution was then heated at $70{ }^{\circ} \mathrm{C}$ for 1.5 hour. The mixture was made basic with $50 \mathrm{~mL}$ of a $10 \%$ ammonia solution and extracted with dichloromethane $(3 \times 40 \mathrm{~mL})$. The organic layer was washed with brine, dried over $\mathrm{Na}_{2} \mathrm{SO}_{4}$, filtered and evaporated under reduced pressure. The crude product was triturated with ethyl acetate to provide 5 as colorless solid (657 $\mathrm{mg}, 94 \%)$, whose spectroscopic characteristics were consistent with those of Herlem-Gaulier, D.; Kuong-HuuLainé, F.; Goutarel, R.; Magdeleine, M.J. Alcaloïdes stéroïdiques LXXII. Alcaloïdes du groupe des cylcloxobuxidines retirés du Buxus balearica Willd : $N$-3-isobutyryl cycloxobuxidine-F (baléabuxidine), N-3-isobutyryl cycloxobuxidine-H, N-3-benzoyl cycloxobuxidine-F et cycloxobuxoxazine-C Bull. Soc. Chim. Fr. 1968, 2, 763-773. mp 242 ${ }^{\circ} \mathrm{C} ;[\alpha]_{\mathrm{D}}=+127\left(\mathrm{c} 0.6,25{ }^{\circ} \mathrm{C}, \mathrm{CHCl}_{3}\right)$. I.R. $\left(\mathrm{CHCl}_{3}\right) \cup$ max $\left(\mathrm{cm}^{-1}\right): 3018,2964,1663,1463$, 1377, 1095. ${ }^{1}$ H R.M.N. (300 MHz, $\mathrm{CDCl}_{3}$ ), $\delta$ ppm : 0.85 (3H, s, H18), 0.88 (3H, d, J = $6.4 \mathrm{~Hz}$, H21), 0.94 (3H, s, H30), 1,00 (1H, m, H6ß), $1.11(1 \mathrm{H}, \mathrm{d}, \mathrm{J}=3.8 \mathrm{~Hz}, \mathrm{H} 19 \alpha), 1.20$ (3H, s, H28), $1.23(1 \mathrm{H}, \mathrm{m}, \mathrm{H} 1 \alpha), 1.29-1.38$ (2H, m, H7), 1.46-1.51 (2H, m, H2 $\beta, \mathrm{H} 15 \alpha), 1.56(1 \mathrm{H}, \mathrm{d}, \mathrm{J}=3.8$

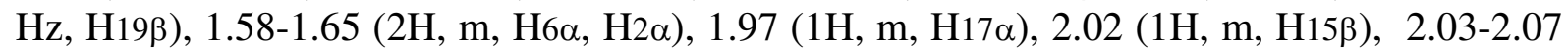

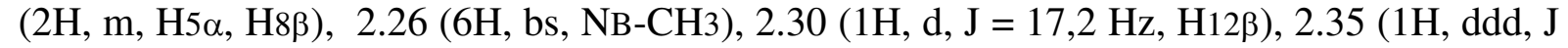
$=3.0 \mathrm{~Hz}, 3.3 \mathrm{~Hz}, 12.6 \mathrm{~Hz}, \mathrm{H} 1 \beta), 2.50(1 \mathrm{H}, \mathrm{d}, \mathrm{J}=17.2 \mathrm{~Hz}, \mathrm{H} 12 \alpha), 2.63(1 \mathrm{H}, \mathrm{dq}, \mathrm{J}=6.4 \mathrm{~Hz}$, $10.9 \mathrm{~Hz}, \mathrm{H} 20), 2.77(1 \mathrm{H}, \mathrm{dd}, \mathrm{J}=4.3 \mathrm{~Hz}, 11,5 \mathrm{~Hz}, \mathrm{H} 3 \alpha), 3.55(1 \mathrm{H}, \mathrm{d}, \mathrm{J}=10.3 \mathrm{~Hz}, \mathrm{H} 29 \mathrm{a}), 3.74$ $(1 \mathrm{H}, \mathrm{d}, \mathrm{J}=10.3 \mathrm{~Hz}, \mathrm{H} 29 \mathrm{~b}), 4.11(1 \mathrm{H}, \mathrm{ddd}, \mathrm{J}=2.8 \mathrm{~Hz}, 7.3 \mathrm{~Hz}, 10.1 \mathrm{~Hz}, \mathrm{H} 16 \beta) .{ }^{13} \mathrm{C}$ R.M.N.

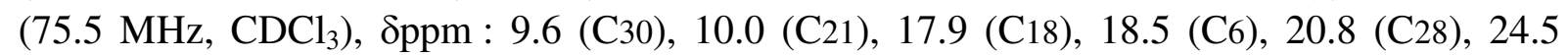
(C7), 27.9 (C1), 30.5 (C19), 34.3 (C2), 37.7 (C10), 41.5 (C8), 42.2 (C4, C9), 42.8 (C15), 44.5 (C13), 45.5 (C5), 47.1 (C14), 51.5 (C12), 55.9 (C17), 59.1 (C3), 62.0 (C20), 73.6 (C29), 78.3 (C16), 211.3 (C11). E.S.I-MS m/z : 433.3 [M+H] (100), 416.3 (10). HRES-MS m/z 433,3425 (calcd for $\mathrm{C}_{26} \mathrm{H}_{45} \mathrm{~N}_{2} \mathrm{O}_{3}=433.3430$ ). 


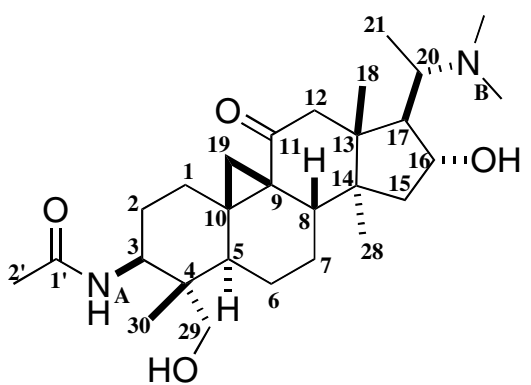

$N-[(3 \beta, 4 \alpha, 5 \alpha, 9 \beta, 16 \alpha, 20 S)-20-($ dimethylamino)-16-hydroxy-4-(hydroxymethyl)-4,14dimethyl-11-oxo-9,19-cyclopregnan-3-yl]acetamide or (+)-N-3-acetylcycloxobuxidine $\mathrm{F}$ (7a).

To a solution of cycloxobuxidine-F 5 (240 mg, $0.55 \mathrm{mmol}, 1$ equiv) in $10 \mathrm{~mL}$ of methanol was added acetic anhydride ( $63 \mu \mathrm{l}, 0.66 \mathrm{mmol}, 1.2$ equiv). After stirring for 5 hours at room temperature, the mixture was neutralized with a few drops of a $10 \%$ sodium bicarbonate solution $(\mathrm{pH}=8)$, then made basic with $50 \mathrm{~mL}$ of a $10 \%$ ammonia solution $(\mathrm{pH}=10)$ and extracted with dichloromethane $(3 \times 40 \mathrm{~mL})$. The organic layer was washed with brine (30 $\mathrm{mL}$ ), dried over $\mathrm{Na}_{2} \mathrm{SO}_{4}$ and filtered, and the solvent was evaporated. The crude product was purified by column chromatography on alumina with dichloromethane-methanol $(98: 2)$ as eluent followed by trituration in acetone to afford a colorless powder of $7 \mathbf{a}(210 \mathrm{mg}, 79 \%)$ : mp $255{ }^{\circ} \mathrm{C} ;[\alpha]^{21}{ }_{\mathrm{D}}=+73\left(\mathrm{c} 0.98, \mathrm{CHCl}_{3}\right) ; \mathrm{IR}\left(\mathrm{CHCl}_{3}\right) \mathrm{v}_{\max }\left(\mathrm{cm}^{-1}\right): 3436,2982,2944,1656$, 1514, 1461, 1372, 1049; ${ }^{1} \mathrm{H}$ NMR $\left(300 \mathrm{MHz}, \mathrm{CDCl}_{3}\right) \delta 0.56(3 \mathrm{H}, \mathrm{s}, \mathrm{H}-30), 0.82(3 \mathrm{H}, \mathrm{s}, \mathrm{H}-$ 18), $0.86(3 \mathrm{H}, \mathrm{d}, J=6.4 \mathrm{~Hz}, \mathrm{H}-21), 0.93(1 \mathrm{H}, \mathrm{m}, \mathrm{H}-6 \beta), 1.03(1 \mathrm{H}, \mathrm{d}, J=3.4 \mathrm{~Hz}, \mathrm{H}-19 \alpha)$, $1.20(3 \mathrm{H}, \mathrm{s}, \mathrm{H}-28), 1.26$ (1H, dd, $J=4.0,13.1 \mathrm{~Hz}, \mathrm{H}-1 \alpha), 1.43(1 \mathrm{H}, \mathrm{m}, \mathrm{H}-7 \alpha), 1.48(1 \mathrm{H}, \mathrm{dd}, J$ $=2.8,14.1 \mathrm{~Hz}, \mathrm{H}-15 \alpha), 1.50(1 \mathrm{H}, \mathrm{m}, \mathrm{H}-7 \beta), 1.55(1 \mathrm{H}, \mathrm{d}, J=3.4 \mathrm{~Hz}, \mathrm{H}-19 \beta), 1.62(1 \mathrm{H}, \mathrm{dd}, J$ $=4.0,13.0 \mathrm{~Hz}, \mathrm{H}-2 \beta), 1.69(2 \mathrm{H}, \mathrm{m}, \mathrm{H}-6 \alpha, \mathrm{H}-2 \alpha), 1.95(1 \mathrm{H}, \mathrm{m}, \mathrm{H}-17 \alpha), 2.00(1 \mathrm{H}, \mathrm{m}, \mathrm{H}-15 \beta)$, $2.08(2 \mathrm{H}, \mathrm{m}, \mathrm{H}-5 \alpha, \mathrm{H}-8 \beta), 2.03$ (3H, s, H-2'), $2.24\left(6 \mathrm{H}, \mathrm{bs}, \mathrm{N}-\mathrm{CH}_{3}\right), 2.28(1 \mathrm{H}, \mathrm{d}, J=17.2 \mathrm{~Hz}$, H-12ß), 2.38 (1H, ddd, $J=3.0,4.0,13.1 \mathrm{~Hz}, \mathrm{H}-1 \beta), 2.51(1 \mathrm{H}, \mathrm{d}, J=17.2 \mathrm{~Hz}, \mathrm{H}-12 \alpha), 2.61$ $(1 \mathrm{H}, \mathrm{dq}, J=6.4,10.9 \mathrm{~Hz}, \mathrm{H}-20), 3.02(1 \mathrm{H}, \mathrm{dd}, J=3.4,12.6 \mathrm{~Hz}, \mathrm{H}-29 \mathrm{a}), 3.34(1 \mathrm{H}, \mathrm{dd}, J=$ $10.9,12.6 \mathrm{~Hz}, \mathrm{H}-29 \mathrm{~b}), 3.98$ (1H, ddd, $J=4.0,8.9,12.3 \mathrm{~Hz}, \mathrm{H}-3 \alpha), 4.09$ (1H, ddd, $J=2.8$, 7.2, $10.0 \mathrm{~Hz}, \mathrm{H}-16 \beta), 4.36(1 \mathrm{H}, \mathrm{dd}, J=3.4,10.9 \mathrm{~Hz}, \mathrm{OH}), 5.67(1 \mathrm{H}, \mathrm{d}, J=8.9 \mathrm{~Hz}, \mathrm{NA} H)$; ${ }^{13} \mathrm{C}$ NMR $\left(75.5 \mathrm{MHz}, \mathrm{CDCl}_{3}\right) \delta 9.9\left(\mathrm{CH}_{3}, \mathrm{C}-21\right), 11.2\left(\mathrm{CH}_{3}, \mathrm{C}-30\right), 17.8\left(\mathrm{CH}_{3}, \mathrm{C}-18\right), 18.4$ $\left(\mathrm{CH}_{2}, \mathrm{C}-6\right), 20.8\left(\mathrm{CH}_{3}, \mathrm{C}-28\right), 23.1\left(\mathrm{CH}_{3}, \mathrm{C}-2\right)$ '), $24.2\left(\mathrm{CH}_{2}, \mathrm{C}-7\right), 27.5\left(\mathrm{CH}_{2}, \mathrm{C}-2\right), 27.6\left(\mathrm{CH}_{2}\right.$, C-1), $30.4\left(\mathrm{CH}_{2}, \mathrm{C}-19\right), 34.4$ (C, C-9), 37.7 (C, C-10), 41.1 (CH, C-5), 41.3 (CH, C-8), 42.7 $\left(\mathrm{CH}_{2}, \mathrm{C}-15\right), 44.4(\mathrm{C}, \mathrm{C}-13), 44.5(\mathrm{C}, \mathrm{C}-4), 47.1(\mathrm{C}, \mathrm{C}-14), 51.3(\mathrm{CH}, \mathrm{C}-3), 51.4\left(\mathrm{CH}_{2}, \mathrm{C}-12\right)$, 55.7 (CH, C-17), 62.0 (CH, C-20), 64.1 ( $\left.\mathrm{CH}_{2}, \mathrm{C}-29\right), 78.3$ (CH, C-16), 171.5 (C, C-1'), 211.5 (C, C-11); ES-MS m/z $475.3[\mathrm{M}+\mathrm{H}]^{+}$(100), 476.4 (15); HRES-MS m/z 475.3511 (calcd for $\left.\mathrm{C}_{28} \mathrm{H}_{47} \mathrm{~N}_{2} \mathrm{O}_{4}, 475.3536\right)$. 


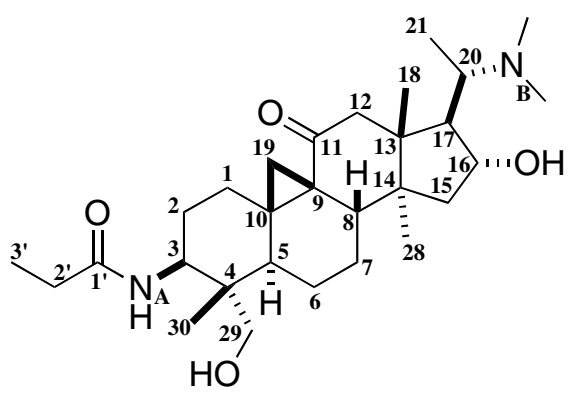

\section{$\mathrm{N}-[(3 \beta, 4 \alpha, 5 \alpha, 9 \beta, 16 \alpha, 20 S)-20-(d i m e t h y l a m i n o)-16-h y d r o x y-4-(h y d r o x y m e t h y l)-4,14-$ dimethyl-11-oxo-9,19-cyclopregnan-3-yl]propanamide (7b)}

To a solution of cycloxobuxidine-F 5 (300 mg, $0.69 \mathrm{mmol}, 1$ equiv) in $10 \mathrm{~mL}$ of methanol was added propionic anhydride $(98 \mu 1,0.76 \mathrm{mmol}, 1.1$ equiv). After stirring for 4 hours at room temperature, the mixture was neutralized with a few drops of a $10 \%$ sodium bicarbonate solution $(\mathrm{pH}=8)$, then made basic with $50 \mathrm{~mL}$ of a $10 \%$ ammonia solution $(\mathrm{pH}=10)$ and extracted with dichloromethane $(3 \times 40 \mathrm{~mL})$. The organic layer was washed with brine (30 $\mathrm{mL}$ ), dried over $\mathrm{Na}_{2} \mathrm{SO}_{4}$ and filtered, and the solvent was evaporated. The crude product was purified by column chromatography on alumina with dichloromethane-methanol (98:2) as eluent followed by trituration in acetone to afford a colorless powder of $7 \mathbf{b}(269 \mathrm{mg}, 80 \%)$ : mp $221{ }^{\circ} \mathrm{C} ;[\alpha]_{D}^{24}=+51\left(\mathrm{c} 0.2, \mathrm{CHCl}_{3}\right) ; \mathrm{IR}\left(\mathrm{CHCl}_{3}\right) \mathrm{v}_{\max }\left(\mathrm{cm}^{-1}\right): 3434,3021,2942,1653$, 1514, 1462, 1380, 1049; ${ }^{1} \mathrm{H}$ NMR (300 MHz, $\left.\mathrm{CDCl}_{3}\right) \delta 0.57(3 \mathrm{H}, \mathrm{s}, \mathrm{H}-30), 0.84(3 \mathrm{H}, \mathrm{s}, \mathrm{H}-$ 18), 0.87 (3H, d, $J=6.4 \mathrm{~Hz}, \mathrm{H}-21), 0.92(1 \mathrm{H}, \mathrm{m}, \mathrm{H}-6 \beta), 1.04(1 \mathrm{H}, \mathrm{d}, J=3.8 \mathrm{~Hz}, \mathrm{H}-19 \alpha)$, $1.18(3 \mathrm{H}, \mathrm{t}, J=7.5 \mathrm{~Hz}, \mathrm{H}-3$ ') $1.22(3 \mathrm{H}, \mathrm{s}, \mathrm{H}-28), 1.40(1 \mathrm{H}, \mathrm{m}, \mathrm{H}-1 \alpha), 1.45(1 \mathrm{H}, \mathrm{m}, \mathrm{H}-7 \alpha)$, $1.50(1 \mathrm{H}, \mathrm{dd}, J=2.6,14.3 \mathrm{~Hz}, \mathrm{H}-15 \alpha), 1.52(1 \mathrm{H}, \mathrm{m}, \mathrm{H}-7 \beta), 1.56(1 \mathrm{H}, \mathrm{d}, J=3.8 \mathrm{~Hz}, \mathrm{H}-19 \beta)$, $1.63(1 \mathrm{H}, \mathrm{dd}, J=4.0,12.8 \mathrm{~Hz}, \mathrm{H}-2 \beta), 1.70(2 \mathrm{H}, \mathrm{m}, \mathrm{H}-6 \alpha, \mathrm{H}-2 \alpha), 1.96$ (1H, m, H-17 $), 2.03$

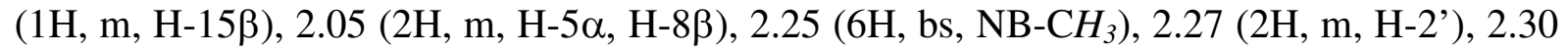
$(1 \mathrm{H}, \mathrm{d}, J=17.1 \mathrm{~Hz}, \mathrm{H}-12 \beta), 2.41(1 \mathrm{H}, \mathrm{ddd}, J=3.0,3.4,13.9 \mathrm{~Hz}, \mathrm{H}-1 \beta), 2.52(1 \mathrm{H}, \mathrm{d}, J=$ $17.1 \mathrm{~Hz}, \mathrm{H}-12 \alpha), 2.63(1 \mathrm{H}, \mathrm{dq}, J=6.4,10.7 \mathrm{~Hz}, \mathrm{H}-20), 3.00(1 \mathrm{H}, \mathrm{dd}, J=4.5,12.6 \mathrm{~Hz}, \mathrm{H}-$ 29a), $3.35(1 \mathrm{H}, \mathrm{dd}, J=10.5,12.6 \mathrm{~Hz}, \mathrm{H}-29 \mathrm{~b}), 4.00$ (1H, ddd, $J=4.2,9.0,12.5 \mathrm{~Hz}, \mathrm{H}-3 \alpha)$, $4.10(1 \mathrm{H}$, ddd, $J=2.6,9.8,10.0 \mathrm{~Hz}, \mathrm{H}-16 \beta), 4.38(1 \mathrm{H}, \mathrm{dd}, J=4.5,10.5 \mathrm{~Hz}, \mathrm{OH}), 5.42(1 \mathrm{H}$, $\mathrm{d}, J=9.0 \mathrm{~Hz}, \mathrm{NAH}) ;{ }^{13} \mathrm{C} \mathrm{NMR}\left(75.5 \mathrm{MHz}, \mathrm{CDCl}_{3}\right) \delta 10.0\left(\mathrm{CH}_{3}, \mathrm{C}-21\right), 10.1\left(\mathrm{CH}_{3}, \mathrm{C}-3{ }^{\prime}\right)$, $11.2\left(\mathrm{CH}_{3}, \mathrm{C}-30\right), 17.9\left(\mathrm{CH}_{3}, \mathrm{C}-18\right), 18.4\left(\mathrm{CH}_{2}, \mathrm{C}-6\right), 20.8\left(\mathrm{CH}_{3}, \mathrm{C}-28\right), 24.3\left(\mathrm{CH}_{2}, \mathrm{C}-7\right), 27.6$ $\left(\mathrm{CH}_{2}, \mathrm{C}-2\right), 27.7\left(\mathrm{CH}_{2}, \mathrm{C}-1\right), 29.8\left(\mathrm{CH}_{2}, \mathrm{C}-2\right)$ ') $30.5\left(\mathrm{CH}_{2}, \mathrm{C}-19\right), 34.5(\mathrm{C}, \mathrm{C}-9), 37.8(\mathrm{C}, \mathrm{C}-$ 10), 41.2 (CH, C-5), 41.4 (CH, C-8), $42.8\left(\mathrm{CH}_{2}, \mathrm{C}-15\right), 44.5$ (C, C-13), 44.5 (C, C-4), 47.2 (C, $\mathrm{C}-14), 51.0(\mathrm{CH}, \mathrm{C}-3), 51.5\left(\mathrm{CH}_{2}, \mathrm{C}-12\right), 55.8(\mathrm{CH}, \mathrm{C}-17), 62.0(\mathrm{CH}, \mathrm{C}-20), 64.2\left(\mathrm{CH}_{2}, \mathrm{C}-\right.$ 29), 78.3 (CH, C-16), 175.2 (C, C-1'), 211.6 (C, C-11); ES-MS m/z $489.3[\mathrm{M}+\mathrm{H}]^{+}$(100), 490.3 (5), 295.2 (15); HRES-MS $m / z 489.3655$ (calcd for $\mathrm{C}_{29} \mathrm{H}_{49} \mathrm{~N}_{2} \mathrm{O}_{4}, 489.3692$ ). 


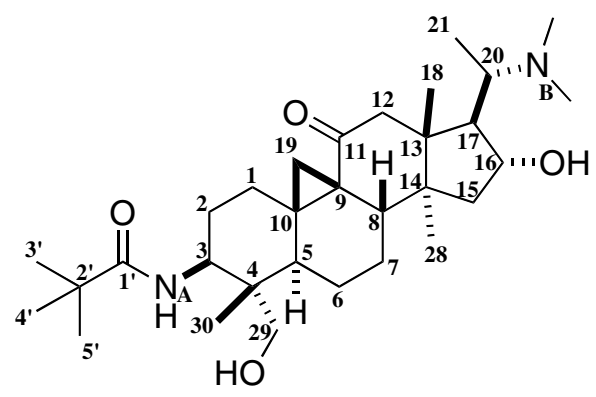

\section{$\mathrm{N}-[(3 \beta, 4 \alpha, 5 \alpha, 16 \alpha, 20 S)-20$-(dimethylamino)-16-hydroxy-4-(hydroxymethyl)-4,14- dimethyl-11-oxo-9,19-cyclopregnan-3-yl]-2,2-dimethylpropanamide $\quad(7 c)$.}

To a solution of cycloxobuxidine-F 5 (300 mg, $0.69 \mathrm{mmol}, 1$ equiv) in $10 \mathrm{~mL}$ of methanol was added pivalic anhydride $(156 \mu \mathrm{l}, 0.76 \mathrm{mmol}, 1.1$ equiv). After stirring for 4 hours at room temperature, the mixture was neutralized with a few drops of a $10 \%$ sodium bicarbonate solution $(\mathrm{pH}=8)$, then made basic with $50 \mathrm{~mL}$ of a $10 \%$ ammonia solution $(\mathrm{pH}=10)$ and extracted with dichloromethane $(3 \times 40 \mathrm{~mL})$. The organic layer was washed with brine (40 $\mathrm{mL}$ ), dried over $\mathrm{Na}_{2} \mathrm{SO}_{4}$ and filtered, and the solvent was evaporated. The crude product was purified by column chromatography on alumina with dichloromethane-methanol $(98: 2)$ as eluent followed by trituration in acetone to afford a colorless powder of 7c (282 $\mathrm{mg}, 79 \%$;): $\operatorname{mp} 267{ }^{\circ} \mathrm{C} ;[\alpha]^{25} \mathrm{D}=+70\left(\mathrm{c} 0.92, \mathrm{CHCl}_{3}\right) ; \mathrm{IR}\left(\mathrm{CHCl}_{3}\right) \mathrm{v}_{\max }\left(\mathrm{cm}^{-1}\right): 3454,2967,1637,1514$, 1461, 1367, 1046; ${ }^{1} \mathrm{H}$ NMR (300 MHz, $\left.\mathrm{CDCl}_{3}\right) \delta 0.55$ (3H, s, H-30), 0.83 (3H, s, H-18), 0.86 $(3 \mathrm{H}, \mathrm{d}, J=6.6 \mathrm{~Hz}, \mathrm{H}-21), 0.93(1 \mathrm{H}, \mathrm{m}, \mathrm{H}-6 \beta), 1.03(1 \mathrm{H}, \mathrm{d}, J=3.8 \mathrm{~Hz}, \mathrm{H}-19 \alpha), 1.21(12 \mathrm{H}, \mathrm{s}$,

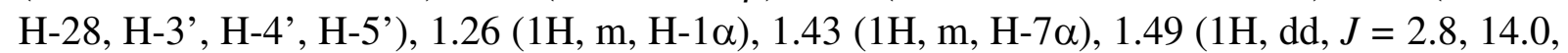
$\mathrm{H}-15 \alpha), 1.53(1 \mathrm{H}, \mathrm{m}, \mathrm{H}-7 \beta), 1.55(1 \mathrm{H}, \mathrm{d}, J=3.8 \mathrm{~Hz}, \mathrm{H}-19 \beta), 1.59(1 \mathrm{H}, \mathrm{dd}, J=3.8,12.4 \mathrm{~Hz}$,

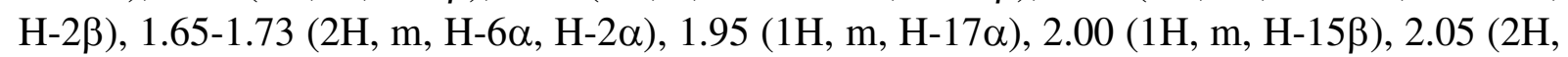
m, H-5 $\alpha, \mathrm{H}-8 \beta), 2.24$ (6H, bs, NB-CH $\left.H_{3}\right), 2.29(1 \mathrm{H}, \mathrm{d}, J=17.3 \mathrm{~Hz}, \mathrm{H}-12 \beta), 2.41$ (1H, ddd, $J$ $=3.4,3.8,13.6 \mathrm{~Hz}, \mathrm{H}-1 \beta), 2.51(1 \mathrm{H}, \mathrm{d}, J=17.3 \mathrm{~Hz}, \mathrm{H}-12 \alpha), 2.60(1 \mathrm{H}, \mathrm{dq}, J=6.6,11.0 \mathrm{~Hz}$, H-20), $2.91(1 \mathrm{H}, \mathrm{dd}, J=4.9,12.6 \mathrm{~Hz}, \mathrm{H}-29 \mathrm{a}), 3.33(1 \mathrm{H}, \mathrm{dd}, J=10.7,12.6 \mathrm{~Hz}, \mathrm{H}-29 \mathrm{~b}), 3.98$ (1H, ddd, $J=4.5,9.0,13.4 \mathrm{~Hz}, \mathrm{H}-3 \alpha), 4.09$ (1H, ddd, $J=2.8 \mathrm{~Hz}, 7.2,10.0 \mathrm{~Hz}, \mathrm{H}-16 \beta), 4.39$ $(1 \mathrm{H}, \mathrm{dd}, J=4.9,10.7 \mathrm{~Hz}, \mathrm{OH}), 5.49(1 \mathrm{H}, \mathrm{d}, J=9.0 \mathrm{~Hz}, \mathrm{NAH}) ;{ }^{13} \mathrm{C} \mathrm{NMR}\left(75.5 \mathrm{MHz}, \mathrm{CDCl}_{3}\right)$ $\delta 9.9\left(\mathrm{CH}_{3}, \mathrm{C}-21\right), 11.2\left(\mathrm{CH}_{3}, \mathrm{C}-30\right), 17,8\left(\mathrm{CH}_{3}, \mathrm{C}-18\right), 18.4\left(\mathrm{CH}_{2}, \mathrm{C}-6\right), 20.8\left(\mathrm{CH}_{3}, \mathrm{C}-28\right)$, $24.3\left(\mathrm{CH}_{2}, \mathrm{C}-7\right), 27.5\left(\mathrm{CH}_{2}, \mathrm{C}-2\right), 27.6\left(\mathrm{CH}_{2}, \mathrm{C}-1\right) 27.6\left(\mathrm{CH}_{3}, \mathrm{C}-3\right.$ ', C-4', C-5'), $30.5\left(\mathrm{CH}_{2}\right.$, C-19), 34.4 (C, C-9), 37.8 (C, C-10), 41.2 (CH, C-5), 41.4 (CH, C-8), $42.8\left(\mathrm{CH}_{2}, \mathrm{C}-15\right), 44.4$ (C, C-13), $44.6(\mathrm{C}, \mathrm{C}-4), 47.1(\mathrm{C}, \mathrm{C}-14), 50.6(\mathrm{CH}, \mathrm{C}-3), 51.5\left(\mathrm{CH}_{2}, \mathrm{C}-12\right), 55.8(\mathrm{CH}, \mathrm{C}-17)$, 62.0 (CH, C-20), $64.1\left(\mathrm{CH}_{2}, \mathrm{C}-29\right), 78.3$ (CH, C-16), 179.9 (C, C-1'), 211.5 (C, C-11); ESMS m/z 517.4 [M+H] $]^{+}(100), 518.5$ (75); HRES-MS $m / z 517.3986\left(\right.$ calcd for $\mathrm{C}_{31} \mathrm{H}_{53} \mathrm{~N}_{2} \mathrm{O}_{4}$, 517.4005). 


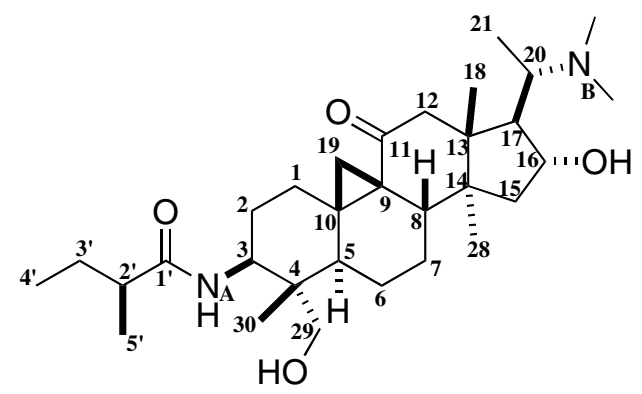

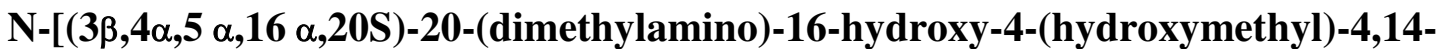 dimethyl-11-oxo-9,19-cyclopregnan-3-yl]-2-methylbutanamide (7d).}

To a solution of cycloxobuxidine-F 5 (300 mg, $0.69 \mathrm{mmol}, 1$ equiv) in $10 \mathrm{~mL}$ of methanol was added (S)-(+)-2-methylbutyric anhydride ( $151 \mu \mathrm{l}, 0.76 \mathrm{mmol}, 1.1$ equiv). After stirring for 3 hours at room temperature, the mixture was neutralized with a few drops of a $10 \%$ sodium bicarbonate solution $(\mathrm{pH}=8)$, then made basic with $50 \mathrm{~mL}$ of a $10 \%$ ammonia solution $(\mathrm{pH}=10)$ and extracted with dichloromethane $(3 \times 40 \mathrm{~mL})$. The organic layer was washed with brine $(30 \mathrm{~mL})$, dried over $\mathrm{Na}_{2} \mathrm{SO}_{4}$ and filtered, and the solvent was evaporated. The crude product was purified by column chromatography on alumina with dichloromethane-methanol $(98: 2)$ as eluent followed by trituration in acetone to afford a colorless powder of 7d $(266 \mathrm{mg}, 75 \%)$ : $\mathrm{mp} 262{ }^{\circ} \mathrm{C}$; $[\alpha]^{21}{ }_{\mathrm{D}}=+59\left(\mathrm{c} 0.44, \mathrm{CHCl}_{3}\right)$; IR $\left(\mathrm{CHCl}_{3}\right) \mathrm{U}_{\max }\left(\mathrm{cm}^{-1}\right): 3454,2968,1651,1511,1461,1382,1042 ;{ }^{1} \mathrm{H}$ NMR $\left(300 \mathrm{MHz}, \mathrm{CDCl}_{3}\right)$ $\delta 0.57(3 \mathrm{H}, \mathrm{s}, \mathrm{H}-30), 0.84(3 \mathrm{H}, \mathrm{s}, \mathrm{H}-18), 0.87(3 \mathrm{H}, \mathrm{d}, J=6.6 \mathrm{~Hz}, \mathrm{H}-21), 0.92(1 \mathrm{H}, \mathrm{m}, \mathrm{H}-6 \beta)$, $0.93(3 \mathrm{H}, \mathrm{t}, J=7.5 \mathrm{~Hz}, \mathrm{H}-4$ ') $1.04(1 \mathrm{H}, \mathrm{d}, J=3.6 \mathrm{~Hz}, \mathrm{H}-19 \alpha), 1.15(3 \mathrm{H}, \mathrm{d}, J=6.8 \mathrm{~Hz}, \mathrm{H}-$ 5'), $1.22(3 \mathrm{H}, \mathrm{s}, \mathrm{H}-28), 1.28(1 \mathrm{H}, \mathrm{m}, \mathrm{H}-1 \alpha), 1.43(1 \mathrm{H}, \mathrm{m}, \mathrm{H}-7 \alpha), 1.45(1 \mathrm{H}, \mathrm{dq}, J=7.5 \mathrm{~Hz}, \mathrm{H}-$ 3'a), $1.50(1 \mathrm{H}, \mathrm{dd}, J=2.9,14.2 \mathrm{~Hz}, \mathrm{H}-15 \alpha), 1.52(1 \mathrm{H}, \mathrm{m}, \mathrm{H}-7 \beta), 1.56(1 \mathrm{H}, \mathrm{d}, J=3.6 \mathrm{~Hz}, \mathrm{H}-$

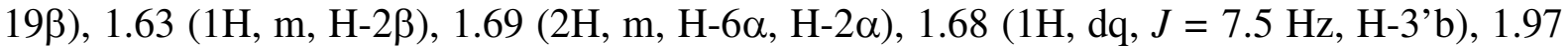

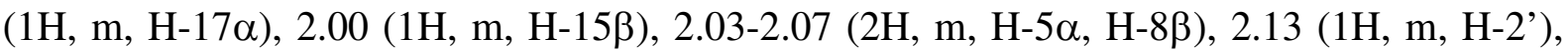
$2.25\left(6 \mathrm{H}, \mathrm{bs}, \mathrm{NB}-\mathrm{CH}_{3}\right), 2.30(1 \mathrm{H}, \mathrm{d}, J=17.1 \mathrm{~Hz}, \mathrm{H}-12 \beta), 2.41(1 \mathrm{H}$, ddd, $J=3.4,3.6 \mathrm{H}, 13.4$ $\mathrm{Hz}, \mathrm{H}-1 \beta), 2.52(1 \mathrm{H}, \mathrm{d}, J=17.1 \mathrm{~Hz}, \mathrm{H}-12 \alpha), 2.62(1 \mathrm{H}, \mathrm{dq}, J=6.6,10.9 \mathrm{~Hz}, \mathrm{H}-20), 3.01(1 \mathrm{H}$, dd, $J=4.9,12.8 \mathrm{~Hz}, \mathrm{H}-29 \mathrm{a}), 3.36$ (1H, dd, $J=10.6,12.8 \mathrm{~Hz}, \mathrm{H}-29 \mathrm{~b}), 4.00$ (1H, ddd, $J=4.3$, 8.9, $12.3 \mathrm{~Hz}, \mathrm{H}-3 \alpha$ ), 4.10 (1H, ddd, $J=2.9,7.1,10.0 \mathrm{~Hz}, \mathrm{H}-16 \beta), 4.46$ (1H, dd, $J=4.9,10.6$ $\mathrm{Hz}, \mathrm{OH}), 5.39(1 \mathrm{H}, \mathrm{d}, J=8.9 \mathrm{~Hz}, \mathrm{NAH}) ;{ }^{13} \mathrm{C} \mathrm{NMR}\left(75.5 \mathrm{MHz}, \mathrm{CDCl}_{3}\right) \delta 9.9\left(\mathrm{CH}_{3}, \mathrm{C}-21\right)$, $11.2\left(\mathrm{CH}_{3}, \mathrm{C}-30\right), 12.1\left(\mathrm{CH}_{3}, \mathrm{C}-4\right.$ '), $17.6\left(\mathrm{CH}_{3}, \mathrm{C}-5\right), 17.8\left(\mathrm{CH}_{3}, \mathrm{C}-18\right), 18.4\left(\mathrm{CH}_{2}, \mathrm{C}-6\right)$, $20.8\left(\mathrm{CH}_{3}, \mathrm{C}-28\right), 24.3\left(\mathrm{CH}_{2}, \mathrm{C} 7\right), 27.5\left(\mathrm{CH}_{2}, \mathrm{C}-2\right), 27.5\left(\mathrm{CH}_{2}, \mathrm{C}-3\right.$ ') $, 27.7\left(\mathrm{CH}_{2}, \mathrm{C}-1\right), 30.6$ $\left(\mathrm{CH}_{2}, \mathrm{C}-19\right), 34.5(\mathrm{C}, \mathrm{C}-9), 37.8(\mathrm{C}, \mathrm{C}-10), 41.1(\mathrm{CH}, \mathrm{C}-5), 41.4(\mathrm{CH}, \mathrm{C}-8), 42.8\left(\mathrm{CH}_{2}, \mathrm{C}-\right.$ 15), 43.5 (CH, C-2'), 44.5 (C, C-13), 44.5 (C, C-4), 47.1 (C, C-14), $51.0(\mathrm{CH}, \mathrm{C}-3), 51.5$ $\left(\mathrm{CH}_{2}, \mathrm{C}-12\right), 55.8(\mathrm{CH}, \mathrm{C}-17), 62.0(\mathrm{CH}, \mathrm{C}-20), 64.2\left(\mathrm{CH}_{2}, \mathrm{C}-29\right), 78.3(\mathrm{CH}, \mathrm{C}-16), 178.0(\mathrm{C}$, C-1'), 211.6 (C, C-11); ES-MS m/z 517.4 [M+H] ${ }^{+}$(100), 518.5 (75); HRES-MS m/z 517.4024 (calcd for $\mathrm{C}_{31} \mathrm{H}_{53} \mathrm{~N}_{2} \mathrm{O}_{4}, 517.4005$ ). 


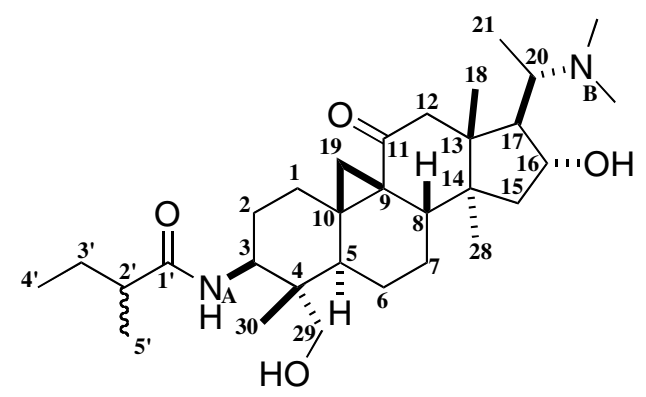

\section{$\mathrm{N}-[(3 \beta, 4 \alpha, 5 \alpha, 16 \alpha, 20 S)-20$-(dimethylamino)-16-hydroxy-4-(hydroxymethyl)-4,14- dimethyl-11-oxo-9,19-cyclopregnan-3-yl]-2-methylbutanamide (7e).}

To a solution of cycloxobuxidine-F 5 (238 mg, $0.55 \mathrm{mmol}, 1$ equiv) in $10 \mathrm{~mL}$ of methanol was added 2-methylbutyric anhydride $(113 \mathrm{mg}, 0.60 \mathrm{mmol}, 1.1$ equiv). After stirring for 5 hours at room temperature, the mixture was neutralized with a few drops of a $10 \%$ sodium bicarbonate solution $(\mathrm{pH}=8)$, then made basic with $50 \mathrm{~mL}$ of a $10 \%$ ammonia solution $(\mathrm{pH}=$ $10)$ and extracted with dichloromethane $(3 \times 40 \mathrm{~mL})$. The organic layer was washed with brine $(30 \mathrm{~mL})$, dried over $\mathrm{Na}_{2} \mathrm{SO}_{4}$ and filtered, and the solvent was evaporated. The crude product was purified by column chromatography on alumina with dichloromethane-methanol (99:1) as eluent followed by trituration in acetone to afford a colorless powder of 7e (238 $\mathrm{mg}, 84 \%)$ : mp $255{ }^{\circ} \mathrm{C}$; IR $\left(\mathrm{CHCl}_{3}\right) v_{\max }\left(\mathrm{cm}^{-1}\right): 3436,3005,2936,1699,1661,1508,1461,1382,1082$; First diastereoisomer ${ }^{1} \mathrm{H}$ NMR $\left(300 \mathrm{MHz}, \mathrm{CDCl}_{3}\right) \delta 0.56(3 \mathrm{H}, \mathrm{s}, \mathrm{H}-30), 0.83(3 \mathrm{H}, \mathrm{s}, \mathrm{H}-18)$, $0.86(3 \mathrm{H}, \mathrm{d}, J=6.6 \mathrm{~Hz}, \mathrm{H}-21), 0.91(3 \mathrm{H}, \mathrm{t}, J=7.2 \mathrm{~Hz}, \mathrm{H}-4$ '), $0.92(1 \mathrm{H}, \mathrm{m}, \mathrm{H}-6 \beta), 1.04(1 \mathrm{H}$, d, $J=3.8 \mathrm{~Hz}, \mathrm{H}-19 \alpha), 1.14$ (3H, d, $J=6.8 \mathrm{~Hz}, \mathrm{H}-5$ '), 1.21 (3H, s, H-28), 1.28 (1H, m, H-1 $\alpha$ ), $1.43(1 \mathrm{H}, \mathrm{m}, \mathrm{H}-7 \alpha), 1.46$ (1H, m, H-3'a), 1.49 (1H, dd, $J=2.6,14.3 \mathrm{~Hz}, \mathrm{H}-15 \alpha), 1.51(1 \mathrm{H}$, m, H-7 $), 1.55$ (1H, d, $J=3.8 \mathrm{~Hz}, \mathrm{H}-19 \beta), 1.60(1 \mathrm{H}, \mathrm{m}, \mathrm{H}-2 \beta), 1.66(2 \mathrm{H}, \mathrm{m}, \mathrm{H}-6 \alpha, \mathrm{H}-2 \alpha)$,

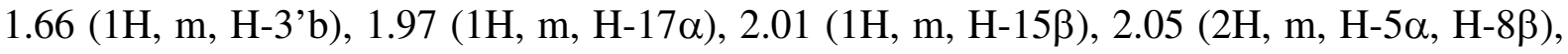
$2.13\left(1 \mathrm{H}, \mathrm{m}, \mathrm{H}-2\right.$ '), $2.24\left(6 \mathrm{H}, \mathrm{bs}, \mathrm{NB}-\mathrm{CH}_{3}\right), 2.29(1 \mathrm{H}, \mathrm{d}, J=17.2 \mathrm{~Hz}, \mathrm{H}-12 \beta), 2.40(1 \mathrm{H}, \mathrm{m}, J$ $=13.6 \mathrm{~Hz}, \mathrm{H}-1 \beta), 2.51(1 \mathrm{H}, \mathrm{d}, J=17.2 \mathrm{~Hz}, \mathrm{H}-12 \alpha), 2.61(1 \mathrm{H}, \mathrm{dq}, J=6.6,10.8 \mathrm{~Hz}, \mathrm{H}-20)$, $2.99(1 \mathrm{H}, \mathrm{m}, J=4.7 \mathrm{~Hz}, \mathrm{H}-29 \mathrm{a}), 3.35(1 \mathrm{H}, \mathrm{m}, J=10.9 \mathrm{~Hz}, \mathrm{H}-29 \mathrm{~b}), 3.99$ (1H, m, H-3a), 4.09 $(1 \mathrm{H}, \mathrm{m}, \mathrm{H}-16 \beta), 4.46(1 \mathrm{H}, \mathrm{m}, J=4.7,10.9 \mathrm{~Hz}, \mathrm{OH}), 5.47(1 \mathrm{H}, \mathrm{d}, J=8.5 \mathrm{~Hz}, \mathrm{NA} H) ;{ }^{13} \mathrm{C}$ NMR (75.5 MHz, CDCl 3$) \delta 9.9\left(\mathrm{CH}_{3}, \mathrm{C}-21\right), 11.2\left(\mathrm{CH}_{3}, \mathrm{C}-30\right), 11.9\left(\mathrm{CH}_{3}, \mathrm{C}-4\right), 17.6\left(\mathrm{CH}_{3}\right.$, C-5'), $17.8\left(\mathrm{CH}_{3}, \mathrm{C}-18\right), 18.4\left(\mathrm{CH}_{2}, \mathrm{C}-6\right), 20.8\left(\mathrm{CH}_{3}, \mathrm{C}-28\right), 24.3\left(\mathrm{CH}_{2}, \mathrm{C}-7\right), 27.5\left(\mathrm{CH}_{2}, \mathrm{C}-\right.$ 2), $27.5\left(\mathrm{CH}_{2}, \mathrm{C}-3\right.$ '), $27.7\left(\mathrm{CH}_{2}, \mathrm{C}-1\right), 30.5\left(\mathrm{CH}_{2}, \mathrm{C}-19\right), 34.4$ (C, C-9), 37.8 (C, C-10), 41.1 $(\mathrm{CH}, \mathrm{C}-5), 41.3(\mathrm{CH}, \mathrm{C}-8), 42.8\left(\mathrm{CH}_{2}, \mathrm{C}-15\right), 43.3(\mathrm{CH}, \mathrm{C}-2$ ') $, 44.4(\mathrm{C}, \mathrm{C}-13), 44.5(\mathrm{C}, \mathrm{C}-4)$, 47.1 (C, C-14), 50.7 (CH, C-3), $51.5\left(\mathrm{CH}_{2}, \mathrm{C}-12\right), 55.8(\mathrm{CH}, \mathrm{C}-17), 62.0(\mathrm{CH}, \mathrm{C}-20), 64.1$ $\left(\mathrm{CH}_{2}, \mathrm{C}-29\right), 78.3(\mathrm{CH}, \mathrm{C}-16), 178.0\left(\mathrm{C}, \mathrm{C}-1\right.$ ') $, 211,6(\mathrm{C}, \mathrm{C}-11)$; Second diastereoisomer ${ }^{1} \mathrm{H}$ NMR $\left(300 \mathrm{MHz}, \mathrm{CDCl}_{3}\right) \delta 0.56(3 \mathrm{H}, \mathrm{s}, \mathrm{H}-30), 0.83(3 \mathrm{H}, \mathrm{s}, \mathrm{H}-18), 0.86(3 \mathrm{H}, \mathrm{d}, J=6.6 \mathrm{~Hz}, \mathrm{H}-$ 21), 0.92 (1H, m, H-6ß), 0.93 (3H, t, $\left.J=7.2 \mathrm{~Hz}, \mathrm{H}-4^{\prime}\right), 1.04$ (1H, d, $\left.J=3.8 \mathrm{~Hz}, \mathrm{H}-19 \alpha\right), 1.16$ $\left(3 \mathrm{H}, \mathrm{d}, J=6.8 \mathrm{~Hz}, \mathrm{H}-5^{\prime}\right), 1.21$ (3H, s, H-28), $1.28(1 \mathrm{H}, \mathrm{m}, \mathrm{H}-1 \alpha), 1.43(1 \mathrm{H}, \mathrm{m}, \mathrm{H}-7 \alpha), 1.46$ (1H, m, H-3'a), 1.49 (1H, dd, $J=2.6,14.3 \mathrm{~Hz}, \mathrm{H}-15 \alpha), 1.51(1 \mathrm{H}, \mathrm{m}, \mathrm{H}-7 \beta), 1.55$ (1H, d, $J=$

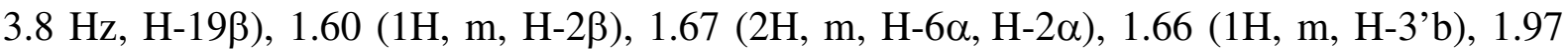

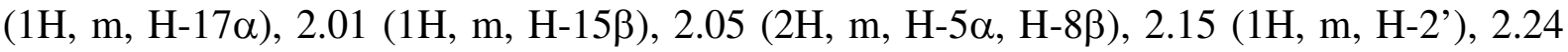
$\left(6 \mathrm{H}, \mathrm{bs}, \mathrm{NB}-\mathrm{CH}_{3}\right), 2.29(1 \mathrm{H}, \mathrm{d}, J=17.2 \mathrm{~Hz}, \mathrm{H}-12 \beta), 2.40(1 \mathrm{H}, \mathrm{m}, J=13.6 \mathrm{~Hz}, \mathrm{H}-1 \beta), 2.51$ $(1 \mathrm{H}, \mathrm{d}, J=17.2 \mathrm{~Hz}, \mathrm{H}-12 \alpha), 2.61(1 \mathrm{H}, \mathrm{dq}, J=6.6,10.8 \mathrm{~Hz}, \mathrm{H}-20), 2.99(1 \mathrm{H}, \mathrm{m}, J=4.7 \mathrm{~Hz}$,

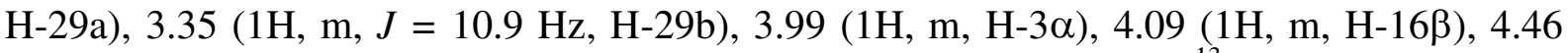
$(1 \mathrm{H}, \mathrm{m}, J=4.7 \mathrm{~Hz}$ et $10.9 \mathrm{~Hz}, \mathrm{OH}), 5.47(1 \mathrm{H}, \mathrm{d}, J=8.5 \mathrm{~Hz}, \mathrm{NAH}) ;{ }^{13} \mathrm{C}$ NMR $(75.5 \mathrm{MHz}$, $\left.\mathrm{CDCl}_{3}\right) \delta 9.9\left(\mathrm{CH}_{3}, \mathrm{C}-21\right), 11.2\left(\mathrm{CH}_{3}, \mathrm{C}-30\right), 12.1\left(\mathrm{CH}_{3}, \mathrm{C}-4^{\prime}\right), 18.1\left(\mathrm{CH}_{3}, \mathrm{C}-5^{\prime}\right), 17.8\left(\mathrm{CH}_{3}\right.$, 
C-18), $18.4\left(\mathrm{CH}_{2}, \mathrm{C}-6\right), 20.8\left(\mathrm{CH}_{3}, \mathrm{C}-28\right), 24.3\left(\mathrm{CH}_{2}, \mathrm{C}-7\right), 27.6\left(\mathrm{CH}_{2}, \mathrm{C}-2\right), 27.6\left(\mathrm{CH}_{2}, \mathrm{C}-\right.$ 3'), $27.7\left(\mathrm{CH}_{2}, \mathrm{C}-1\right), 30.6\left(\mathrm{CH}_{2}, \mathrm{C}-19\right), 34.4$ (C, C-9), 37.8 (C, C-10), $41.2(\mathrm{CH}, \mathrm{C} 5), 41.4$ (CH, C8), $42.8\left(\mathrm{CH}_{2}, \mathrm{C}-15\right), 43.5(\mathrm{CH}, \mathrm{C}-2)$ ') 44.4 (C, C-13), 44.5 (C, C-4), 47.1 (C, C-14), $51.0(\mathrm{CH}, \mathrm{C}-3), 51.5\left(\mathrm{CH}_{2}, \mathrm{C}-12\right), 55.8(\mathrm{CH}, \mathrm{C}-17), 62.0(\mathrm{CH}, \mathrm{C}-20), 64.2\left(\mathrm{CH}_{2}, \mathrm{C}-29\right), 78.3$ (CH, C-16), 178.0 (C, C-1'), 211.6 (C, C-11); ES-MS m/z $517.4[\mathrm{M}+\mathrm{H}]^{+}(100), 518.4$ (70); HRES-MS $m / z 517.4001$ (calcd for $\mathrm{C}_{31} \mathrm{H}_{53} \mathrm{~N}_{2} \mathrm{O}_{4}, 517.4005$ ).

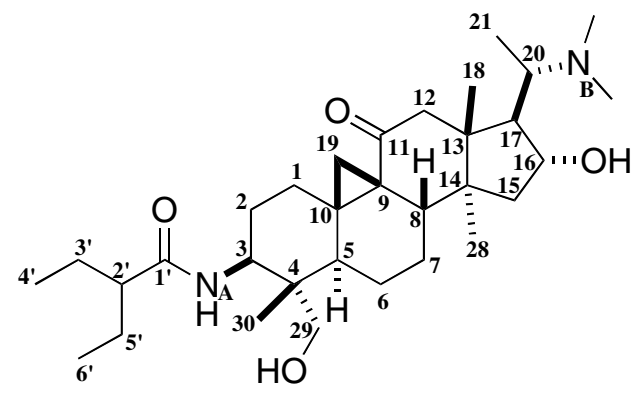

\section{$\mathrm{N}-[(3 \beta, 4 \alpha, 5 \alpha, 16 \alpha, 20 S)-20$-(dimethylamino)-16-hydroxy-4-(hydroxymethyl)-4,14- dimethyl-11-oxo-9,19-cyclopregnan-3-yl]-2-ethylbutanamide (7f).}

To a solution of cycloxobuxidine-F 5 (207 mg, $0.48 \mathrm{mmol}, 1$ equiv) in $10 \mathrm{~mL}$ of methanol was added 2-ethylbutyric anhydride ( $113 \mathrm{mg}, 0.53 \mathrm{mmol}, 1.1$ equiv). After stirring for 5 hours at room temperature, the mixture was neutralized with a few drops of a $10 \%$ sodium bicarbonate solution $(\mathrm{pH}=8)$, then made basic with $50 \mathrm{~mL}$ of a $10 \%$ ammonia solution $(\mathrm{pH}=$ $10)$ and extracted with dichloromethane $(3 \times 40 \mathrm{~mL})$. The organic layer was washed with brine $(30 \mathrm{~mL})$, dried over $\mathrm{Na}_{2} \mathrm{SO}_{4}$ and filtered, and the solvent was evaporated. The crude product was purified by column chromatography on alumina with dichloromethane-methanol (99:1) as eluent followed by trituration in acetone to afford a colorless powder of $7 \mathbf{f}(206 \mathrm{mg}, 81 \%)$ : $\operatorname{mp~} 251{ }^{\circ} \mathrm{C} ;[\alpha]^{20}{ }_{\mathrm{D}}=+63\left(\mathrm{c} 1.07, \mathrm{CHCl}_{3}\right) ; \mathrm{IR}\left(\mathrm{CHCl}_{3}\right) \mathrm{v}_{\max }\left(\mathrm{cm}^{-1}\right): 3433,2967,1690,1650$, 1511, 1461, 1382, 1048; ${ }^{1} \mathrm{H}$ NMR $\left(300 \mathrm{MHz}, \mathrm{CDCl}_{3}\right) \delta 0.58(3 \mathrm{H}, \mathrm{s}, \mathrm{H}-30), 0.86(3 \mathrm{H}, \mathrm{d}, J=$ $6.6 \mathrm{~Hz}, \mathrm{H}-21), 0.89$ (3H, s, H-18), 0.91, 0.93 (6H, 2t, J = $7.3 \mathrm{~Hz}, \mathrm{H}-4$ ', H-6'), 0.95 (1H, m, H$6 \beta), 1.05(1 \mathrm{H}, \mathrm{d}, J=3.5 \mathrm{~Hz}, \mathrm{H}-19 \alpha), 1.23(3 \mathrm{H}, \mathrm{s}, \mathrm{H}-28), 1.33(1 \mathrm{H}, \mathrm{m}, \mathrm{H}-1 \alpha), 1.46(1 \mathrm{H}, \mathrm{m}$, H-7 $\alpha), 1.51(1 \mathrm{H}, \mathrm{dd}, J=2.8,14.0 \mathrm{~Hz}, \mathrm{H}-15 \alpha), 1.53$ (2H, m, H-3'a, H-5'a), 1.55 (1H, m, H-

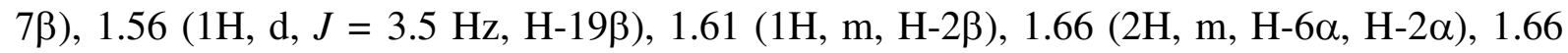

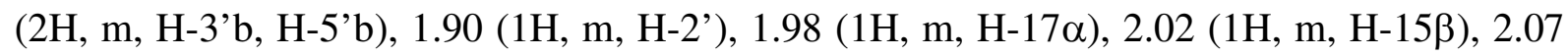
$(2 \mathrm{H}, \mathrm{m}, \mathrm{H}-5 \alpha, \mathrm{H}-8 \beta), 2.25\left(6 \mathrm{H}, \mathrm{bs}, \mathrm{NB}-\mathrm{CH}_{3}\right), 2.31(1 \mathrm{H}, \mathrm{d}, J=17.2 \mathrm{~Hz}, \mathrm{H}-12 \beta), 2.42(1 \mathrm{H}$, ddd, $J=3.2,3.2,13.2 \mathrm{~Hz}, \mathrm{H}-1 \beta), 2.53(1 \mathrm{H}, \mathrm{d}, J=17.2 \mathrm{~Hz}, \mathrm{H}-12 \alpha), 2.62(1 \mathrm{H}, \mathrm{dq}, J=6.6$, $10.7 \mathrm{~Hz}, \mathrm{H}-20), 3.05(1 \mathrm{H}, \mathrm{dd}, J=4.7,12.8 \mathrm{~Hz}, \mathrm{H}-29 \mathrm{a}), 3.38(1 \mathrm{H}, \mathrm{dd}, J=11.5,12.8 \mathrm{~Hz}, \mathrm{H}-$

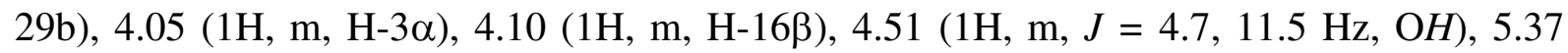
$(1 \mathrm{H}, \mathrm{d}, J=8.9 \mathrm{~Hz}, \mathrm{NAH}) ;{ }^{13} \mathrm{C} \mathrm{NMR}\left(75.5 \mathrm{MHz}, \mathrm{CDCl}_{3}\right) \delta 10.0\left(\mathrm{CH}_{3}, \mathrm{C}-21\right), 11.3\left(\mathrm{CH}_{3}, \mathrm{C}-\right.$ 30), 12.1, $12.4\left(\mathrm{CH}_{3}, \mathrm{C} 4\right.$ ', C6'), $17.9\left(\mathrm{CH}_{3}, \mathrm{C}-18\right), 18.4\left(\mathrm{CH}_{2}, \mathrm{C}-6\right), 20.9\left(\mathrm{CH}_{3}, \mathrm{C}-28\right), 24.3$ $\left(\mathrm{CH}_{2}, \mathrm{C}-7\right), 25.8,26.0\left(\mathrm{CH}_{2}, \mathrm{C}-3^{\prime}, \mathrm{C}-5\right), 27.7\left(\mathrm{CH}_{2}, \mathrm{C}-1\right), 28.0\left(\mathrm{CH}_{2}, \mathrm{C}-2\right), 30.5\left(\mathrm{CH}_{2}, \mathrm{C}-19\right)$, 34.5 (C, C-9), 37.8 (C, C-10), 41.1 (CH, C-5), $41.4(\mathrm{CH}, \mathrm{C} 8), 42.8\left(\mathrm{CH}_{2}, \mathrm{C}-15\right), 44.4$ (C, C13), 44.5 (C, C-4), 47.2 (C, C-14), 51.2 (CH, C-3), $51.5\left(\mathrm{CH}_{2}, \mathrm{C}-12\right), 51.8(\mathrm{CH}, \mathrm{C}-2$ '), 55.8 (CH, C-17), 62.0 (CH, C-20), $64.3\left(\mathrm{CH}_{2}, \mathrm{C}-29\right), 78.3$ (CH, C-16), 177.3 (C, C-1'), 211.6 (C, $\mathrm{C}-11$ ); ES-MS $m / z 531.3$ [M+H] $^{+}$(100), 532.3 (10); HRES-MS m/z 531.4163 (calcd for $\mathrm{C}_{32} \mathrm{H}_{55} \mathrm{~N}_{2} \mathrm{O}_{4}, 531.4162$ ). 


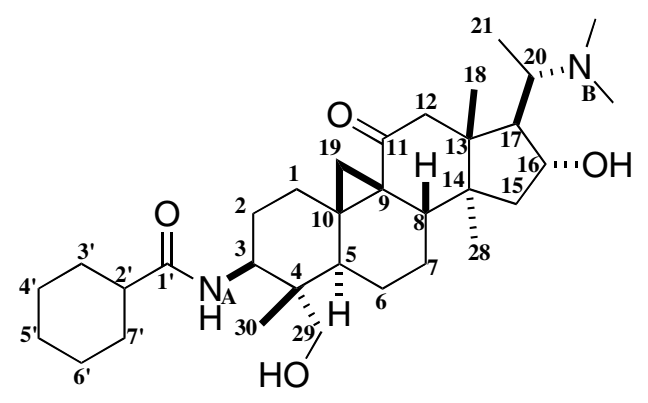

\section{$\mathrm{N}-[(3 \beta, 4 \alpha, 5 \alpha, 16 \alpha, 20 S)-20$-(dimethylamino)-16-hydroxy-4-(hydroxymethyl)-4,14- dimethyl-11-oxo-9,19-cyclopregnan-3-yl]cyclohexanecarboxamide $(7 h)$.}

To a solution of cycloxobuxidine-F 5 (300 mg, $0.69 \mathrm{mmol}, 1$ equiv) in $10 \mathrm{~mL}$ of methanol was added cyclohexanecarboxylic anhydride (182 $\mathrm{mg}, 0.76 \mathrm{mmol}, 1.1 \mathrm{equiv})$. After stirring during 4 hours at room temperature, the mixture was neutralized with a few drops of a $10 \%$ sodium bicarbonate solution $(\mathrm{pH}=8)$, then made basic with $50 \mathrm{~mL}$ of a $10 \%$ ammonia solution $(\mathrm{pH}=10)$ and extracted with dichloromethane $(3 \times 40 \mathrm{~mL})$. The organic layer was washed with brine $(30 \mathrm{~mL})$, dried over $\mathrm{Na}_{2} \mathrm{SO}_{4}$ and filtered, and the solvent was evaporated. The crude product was purified by column chromatography on alumina with dichloromethane-methanol (98:2) as eluent followed by trituration in acetone to afford a colorless powder of $7 \mathbf{h}(225 \mathrm{mg}, 68 \%)$ : mp $293{ }^{\circ} \mathrm{C}$; $[\alpha]^{25}{ }_{\mathrm{D}}=+62\left(\mathrm{c} 1.09, \mathrm{CHCl}_{3}\right)$; IR $\left(\mathrm{CHCl}_{3}\right) \cup_{\max }\left(\mathrm{cm}^{-1}\right): 3434,2937,1650,1512,1452,1382,1050 ;{ }^{1} \mathrm{H}$ NMR $\left(300 \mathrm{MHz}, \mathrm{CDCl}_{3}\right)$ $\delta 0.56(3 \mathrm{H}, \mathrm{s}, \mathrm{H}-30), 0.84(3 \mathrm{H}, \mathrm{s}, \mathrm{H}-18), 0.87(3 \mathrm{H}, \mathrm{d}, J=6.6 \mathrm{~Hz}, \mathrm{H}-21), 0.91(1 \mathrm{H}, \mathrm{m}, \mathrm{H}-6 \beta)$, $1.04(1 \mathrm{H}, \mathrm{d}, J=3.8 \mathrm{~Hz}, \mathrm{H}-19 \alpha), 1.22(3 \mathrm{H}, \mathrm{s}, \mathrm{H}-28), 1.28(1 \mathrm{H}, \mathrm{m}, \mathrm{H}-1 \alpha), 1.37$ (6H, m, H-3', H-7', H-5', H-7 $\alpha), 1.50$ (1H, dd, $J=2.8,14.1 \mathrm{~Hz}, \mathrm{H}-15 \alpha), 1.51(1 \mathrm{H}, \mathrm{m}, \mathrm{H}-7 \beta), 1.56(1 \mathrm{H}, \mathrm{d}, J$

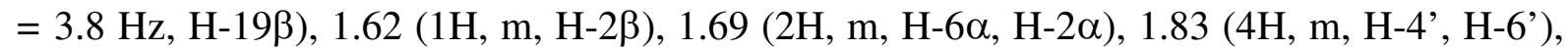

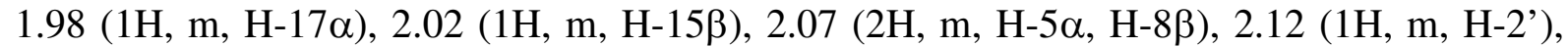
$2.25\left(6 \mathrm{H}, \mathrm{bs}, \mathrm{NB}-\mathrm{CH}_{3}\right), 2.30(1 \mathrm{H}, \mathrm{d}, J=17.1 \mathrm{~Hz}, \mathrm{H}-12 \beta), 2.41(1 \mathrm{H}$, ddd, $J=3.4,3.6,13.4$ $\mathrm{Hz}, \mathrm{H}-1 \beta), 2.52(1 \mathrm{H}, \mathrm{d}, J=17.1 \mathrm{~Hz}, \mathrm{H}-12 \alpha), 2.62(1 \mathrm{H}, \mathrm{dq}, J=6.4,10.7 \mathrm{~Hz}, \mathrm{H}-20), 2.96(1 \mathrm{H}$, $\mathrm{dd}, J=4.9,12.7 \mathrm{~Hz}, \mathrm{H}-29 \mathrm{a}), 3.34(1 \mathrm{H}, \mathrm{dd}, J=10.6,12.7 \mathrm{~Hz}, \mathrm{H}-29 \mathrm{~b}), 3.99$ (1H, ddd, $J=4.3$, 8.9, $12.1 \mathrm{~Hz}, \mathrm{H}-3 \alpha), 4.10$ (1H, ddd, $J=2.6,9.8,10.0 \mathrm{~Hz}, \mathrm{H}-16 \beta), 4.41(1 \mathrm{H}, \mathrm{dd}, J=4.9,10.6$ $\mathrm{Hz}, \mathrm{OH}), 5.36(1 \mathrm{H}, \mathrm{d}, J=8.9 \mathrm{~Hz}, \mathrm{NAH}) ;{ }^{13} \mathrm{C} \mathrm{NMR}\left(75.5 \mathrm{MHz}, \mathrm{CDCl}_{3}\right) \delta 10.0\left(\mathrm{CH}_{3}, \mathrm{C}-21\right)$, $11.2\left(\mathrm{CH}_{3}, \mathrm{C}-30\right), 17.9\left(\mathrm{CH}_{3}, \mathrm{C}-18\right), 18.4\left(\mathrm{CH}_{2}, \mathrm{C}-6\right), 20.9\left(\mathrm{CH}_{3}, \mathrm{C}-28\right), 24.3\left(\mathrm{CH}_{2}, \mathrm{C} 7\right), 25.6$, $25.7\left(\mathrm{CH}_{2}, \mathrm{C}-4\right.$ ', C-6'), $27.6\left(\mathrm{CH}_{2}, \mathrm{C}-2\right), 27.7\left(\mathrm{CH}_{2}, \mathrm{C}-1\right), 29.5\left(\mathrm{CH}_{2}, \mathrm{C}-5\right.$ ' $), 30.3\left(\mathrm{CH}_{2}, \mathrm{C}-3\right.$ ' $)$, $30.3\left(\mathrm{CH}_{2}, \mathrm{C}-7\right.$ '), $30.6\left(\mathrm{CH}_{2}, \mathrm{C}-19\right), 34.5$ (C, C-9), 37.8 (C, C-10), $41.2(\mathrm{CH}, \mathrm{C}-5), 41.4(\mathrm{CH}$, C-8), $42.8\left(\mathrm{CH}_{2}, \mathrm{C}-15\right), 44.5$ (C, C-13, C-4), 45.6 (CH, C-2'), 47.2 (C, C-14), 50.6 (CH, C-3), $51.5\left(\mathrm{CH}_{2}, \mathrm{C}-12\right), 55.8(\mathrm{CH}, \mathrm{C}-17), 62.1(\mathrm{CH}, \mathrm{C}-20), 64.2\left(\mathrm{CH}_{2}, \mathrm{C}-29\right), 78.3(\mathrm{CH}, \mathrm{C}-16)$, 177.6 (C, C-1'), 211.6 (C, C-11); ES-MS m/z $543.4[\mathrm{M}+\mathrm{H}]^{+}(100), 544.5$ (85); HRES-MS m/z 543.4128 (calcd for $\mathrm{C}_{33} \mathrm{H}_{55} \mathrm{~N}_{2} \mathrm{O}_{4}, 543.4162$ ). 


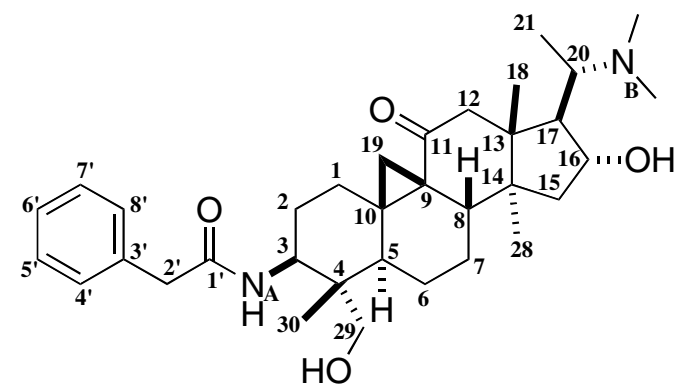

\section{$\mathrm{N}-[(3 \beta, 4 \alpha, 5 \alpha, 16 \mathrm{a}, 20 \mathrm{~S})-20$-(dimethylamino)-16-hydroxy-4-(hydroxymethyl)-4,14- dimethyl-11-oxo-9,19-cyclopregnan-3-yl]benzamide (7i).}

To a solution of cycloxobuxidine-F 5 (300 mg, $0.69 \mathrm{mmol}, 1$ equiv) in $10 \mathrm{~mL}$ of methanol was added a suspension of phenylacetic anhydride $(194 \mathrm{mg}, 0.76 \mathrm{mmol}, 1.1$ equiv) in $5 \mathrm{ml}$ of methanol. After stirring for 4 hours at room temperature, the mixture was neutralized with a few drops of a $10 \%$ sodium bicarbonate solution $(\mathrm{pH}=8)$, then made basic with $50 \mathrm{~mL}$ of a $10 \%$ ammonia solution $(\mathrm{pH}=10)$ and extracted with dichloromethane $(3 \times 40 \mathrm{~mL})$. The organic layer was washed with brine $(30 \mathrm{~mL})$, dried over $\mathrm{Na}_{2} \mathrm{SO}_{4}$ and filtered, and the solvent was evaporated. The crude product was purified by column chromatography on alumina with dichloromethane-methanol $(98: 2)$ as eluent followed by trituration in acetone to afford a colorless powder of $7 \mathbf{i}(255 \mathrm{mg}, 67 \%): \mathrm{mp} 265^{\circ} \mathrm{C} ;[\alpha]_{\mathrm{D}}^{25}=+62\left(\mathrm{c} 0.84, \mathrm{CHCl}_{3}\right) ; \mathrm{IR}\left(\mathrm{CHCl}_{3}\right)$ $\mathrm{U}_{\max }\left(\mathrm{cm}^{-1}\right): 3413,3005,2944,1650,1516,1454,1384,1047 ;{ }^{1} \mathrm{H}$ NMR $\left(300 \mathrm{MHz}, \mathrm{CDCl}_{3}\right) \delta$

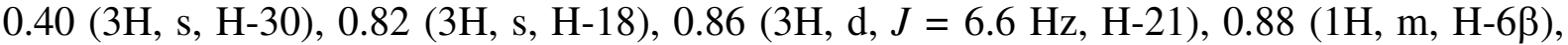
$0.99(1 \mathrm{H}, \mathrm{d}, J=3.6 \mathrm{~Hz}, \mathrm{H}-19 \alpha), 1.20$ (3H, s, H-28), $1.26(1 \mathrm{H}, \mathrm{m}, \mathrm{H}-1 \alpha), 1.40(1 \mathrm{H}, \mathrm{m}, \mathrm{H}-7 \alpha)$, $1.48(1 \mathrm{H}, \mathrm{dd}, J=2.8,13.7 \mathrm{~Hz}, \mathrm{H}-15 \alpha), 1.50(1 \mathrm{H}, \mathrm{m}, \mathrm{H}-7 \beta), 1.51(1 \mathrm{H}, \mathrm{d}, J=3.6 \mathrm{~Hz}, \mathrm{H}-19 \beta)$, $1.57(1 \mathrm{H}, \mathrm{m}, \mathrm{H}-2 \beta), 1.64(1 \mathrm{H}, \mathrm{m}, \mathrm{H}-2 \alpha), 1.68(1 \mathrm{H}, \mathrm{dd}, J=3.6,13.2 \mathrm{~Hz}, \mathrm{H}-6 \alpha), 1.95(1 \mathrm{H}, \mathrm{m}$,

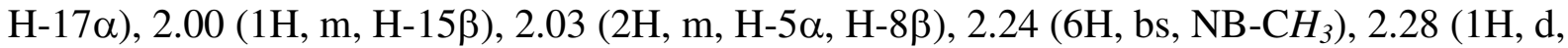
$J=17.2 \mathrm{~Hz}, \mathrm{H}-12 \beta), 2.37(1 \mathrm{H}, \mathrm{ddd}, J=3.2,3.4,13.4 \mathrm{~Hz}, \mathrm{H}-1 \beta), 2.50(1 \mathrm{H}, \mathrm{d}, J=17.2 \mathrm{~Hz}$, H-12 $\alpha), 2.61(1 \mathrm{H}, \mathrm{dq}, J=6.6,10.9 \mathrm{~Hz}, \mathrm{H}-20), 2.94(1 \mathrm{H}, \mathrm{dd}, J=5.0,12.8 \mathrm{~Hz}, \mathrm{H}-29 \mathrm{a}), 3.32$ $(1 \mathrm{H}, \mathrm{dd}, J=10.5,12.8 \mathrm{~Hz}, \mathrm{H}-29 \mathrm{~b}), 3.58,3.63(2 \mathrm{H}, 2 \mathrm{~d}, J=16.0 \mathrm{~Hz}, \mathrm{H}-2$ '), 3.99 (1H, ddd, $J=$ $4.1,9.0,12.2 \mathrm{~Hz}, \mathrm{H}-3 \alpha), 4.09$ (1H, ddd, $J=2.6,7.2,10.2 \mathrm{~Hz}, \mathrm{H}-16 \beta), 4.24(1 \mathrm{H}, \mathrm{dd}, J=5.0$, $10.5 \mathrm{~Hz}, \mathrm{OH}), 5.34(1 \mathrm{H}, \mathrm{d}, J=9.0 \mathrm{~Hz}, \mathrm{NAH}), 7,31\left(5 \mathrm{H}, \mathrm{m}, \mathrm{HAr}=4^{\prime}, 5^{\prime}, 6^{\prime}, 7^{\prime}, 8^{\prime}\right) ;{ }^{13} \mathrm{C} \mathrm{NMR}$ (75.5 MHz, $\left.\mathrm{CDCl}_{3}\right) \delta 9.9\left(\mathrm{CH}_{3}, \mathrm{C}-21\right), 11.0\left(\mathrm{CH}_{3}, \mathrm{C}-30\right), 17.8\left(\mathrm{CH}_{3}, \mathrm{C}-18\right), 18.4\left(\mathrm{CH}_{2}, \mathrm{C}-6\right)$, $20.8\left(\mathrm{CH}_{3}, \mathrm{C}-28\right), 24.3\left(\mathrm{CH}_{2}, \mathrm{C}-7\right), 27.3\left(\mathrm{CH}_{2}, \mathrm{C}-2\right), 27.5\left(\mathrm{CH}_{2}, \mathrm{C}-1\right), 30.5\left(\mathrm{CH}_{2}, \mathrm{C}-19\right), 34.4$ (C, C-9), 37.6 (C, C-10), $41.1(\mathrm{CH}, \mathrm{C}-5), 41.4(\mathrm{CH}, \mathrm{C}-8), 42.8\left(\mathrm{CH}_{2}, \mathrm{C}-15\right), 43.6$ (CH, C-2'), 44.4 (C, C-13), 44.5 (C, C-4), 47.1 (C, C-14), $51.1(\mathrm{CH}, \mathrm{C}-3), 51.5\left(\mathrm{CH}_{2}, \mathrm{C}-12\right), 55.8(\mathrm{CH}, \mathrm{C}-$ 17), 62.0 (CH, C-20), 64.1 ( $\left.\mathrm{CH}_{2}, \mathrm{C}-29\right), 78.3(\mathrm{CH}, \mathrm{C}-16), 127.6(\mathrm{CH}, \mathrm{C}-6$ ') $, 129.1,129.2$ (C$\left.\mathrm{Ar}=4^{\prime}, 5^{\prime}, 7^{\prime}, 8^{\prime}\right), 134.5\left(\mathrm{C}, \mathrm{C}-3^{\prime}\right), 172.4\left(\mathrm{C}, \mathrm{C}^{\prime} 1^{\prime}\right), 211.5(\mathrm{C}, \mathrm{C}-11)$; ES-MS m/z $551.4[\mathrm{M}+\mathrm{H}]^{+}$ (100), 552.5 (90), 553.5 (10); HRES-MS $m / z 551.3816$ (calcd for $\mathrm{C}_{34} \mathrm{H}_{51} \mathrm{~N}_{2} \mathrm{O}_{4}, 551.3849$ ). 


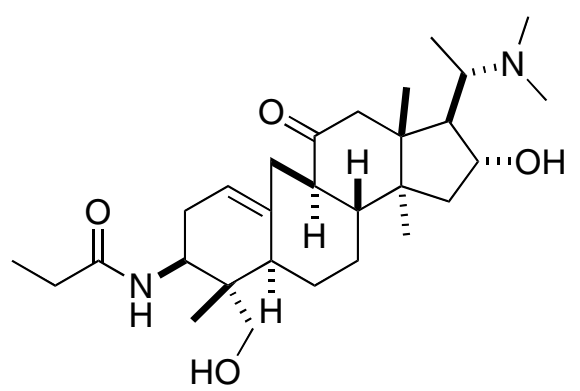

$N-[(2 R, 3 S, 3 \mathrm{a} R, 5 \mathrm{a} R, 9 S, 10 S, 10 \mathrm{a} R, 12 \mathrm{a} R, 12 \mathrm{~b} S)-3-[1-($ dimethylamino)ethyl]-2-hydroxy-10 -(hydroxymethyl)-3a,10,12b-trimethyl-5-oxo-1,2,3,3a,4,5,5a,6,8,9,10,10a,11,12,12 a,12b-hexadecahydrobenzo[4,5]cyclohepta[1,2-e]inden-9-yl]propanamide $(8 b)$

Compound $7 \mathbf{b}$ (73 mg, $0.15 \mathrm{mmol}, 1$ equiv) was heated at $240{ }^{\circ} \mathrm{C}$ under $0.03 \mathrm{mmHg}$ in a rotating distillation oven with four bulbs. A product sublimated in 3 hours. The sublimate was composed of a mixture of the expected product $\mathbf{8 b}(64 \%)$ and dihydro-oxazine $\mathbf{9 b}(36 \%)$. The sublimate was purified by column chromatography on alumina with dichloromethanemethanol (99.7:0.3) as eluent followed by trituration in acetone to afford a colorless powder of $\mathbf{8 b}(51.6 \mathrm{mg}, 71 \%): \mathrm{mp} 229^{\circ} \mathrm{C}$; IR $\left(\mathrm{CHCl}_{3}\right) v_{\max }\left(\mathrm{cm}^{-1}\right): 3433,2980,1693,1653,1514$, 1462, 1379, 1044; ${ }^{1} \mathrm{H}$ NMR (300 MHz, $\left.\mathrm{CDCl}_{3}\right) \delta 0.47$ (3H, s, H-30), 0.69 (3H, s, H-18), 0.87 $(3 \mathrm{H}, \mathrm{d}, J=6.2 \mathrm{~Hz}, \mathrm{H}-21), 1.19$ (3H, t, J= 7.7 Hz, H-3'), 1.27 (3H, s, H-28), 1.29 (1H, m, H$6 \beta), 1.51(1 \mathrm{H}, \mathrm{dd}, J=1.7,13.8 \mathrm{~Hz}, \mathrm{H}-15 \alpha), 1.54(1 \mathrm{H}, \mathrm{bd}, J=10.9 \mathrm{~Hz}, \mathrm{H}-7 \alpha), 1.64(1 \mathrm{H}, \mathrm{m}$,

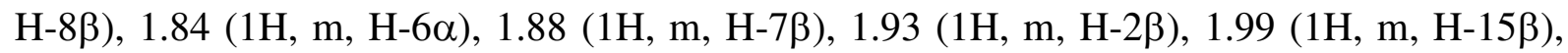
$2.03(1 \mathrm{H}, \mathrm{dd}, J=7.0,11.0 \mathrm{~Hz}, \mathrm{H}-17 \alpha), 2.04(1 \mathrm{H}, \mathrm{m}, \mathrm{H}-9 \alpha), 2.08(1 \mathrm{H}, \mathrm{m}, \mathrm{H}-19 \beta), 2.27(6 \mathrm{H}$,

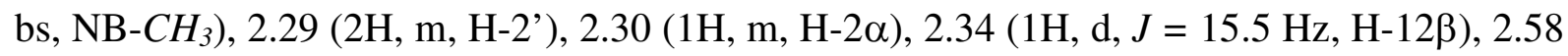

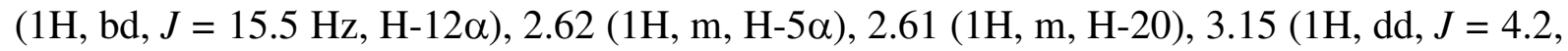
$12.5 \mathrm{~Hz}, \mathrm{H}-29 \mathrm{a}), 3.35$ (1H, d, $J=13.8 \mathrm{~Hz}, \mathrm{H}-19 \alpha), 3.41$ (1H, m, $J=10.7 \mathrm{~Hz}, \mathrm{H}-29 \mathrm{~b}), 4.09$

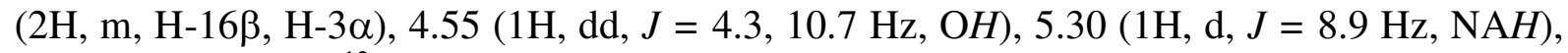
$5.47(1 \mathrm{H}, \mathrm{m}, \mathrm{H}-1) ;{ }^{13} \mathrm{C}$ NMR $\left(75.5 \mathrm{MHz}, \mathrm{CDCl}_{3}\right) \delta 9.9\left(\mathrm{CH}_{3}, \mathrm{C}-21\right), 10.0\left(\mathrm{CH}_{3}, \mathrm{C}-30\right), 10.1$ $\left(\mathrm{CH}_{3}, \mathrm{C}-3\right.$ ') $17.8\left(\mathrm{CH}_{3}, \mathrm{C}-18\right), 19.0\left(\mathrm{CH}_{3}, \mathrm{C}-28\right), 25.0\left(\mathrm{CH}_{2}, \mathrm{C}-6\right), 29.8\left(\mathrm{CH}_{2}, \mathrm{C}-2\right), 30.0$ $\left(\mathrm{CH}_{2}, \mathrm{C}-2\right), 33.5\left(\mathrm{CH}_{2}, \mathrm{C}-7\right), 37.5\left(\mathrm{CH}_{2}, \mathrm{C}-19\right), 42.0(\mathrm{C}, \mathrm{C}-4), 42.4\left(\mathrm{CH}_{2}, \mathrm{C}-15\right), 43.4(\mathrm{CH}, \mathrm{C}-$ 5), 46.2 (C, C-14), 47.1 (C, C-13), 48.1 (CH, C-3), 49.9 (CH, C-8), $50.1\left(\mathrm{CH}_{2}, \mathrm{C}-12\right), 50.2$ (CH, C-9), $55.4(\mathrm{CH}, \mathrm{C}-17), 62.0(\mathrm{CH}, \mathrm{C}-20), 63.8\left(\mathrm{CH}_{2}, \mathrm{C}-29\right), 78.4(\mathrm{CH}, \mathrm{C}-16), 117.9(\mathrm{CH}$, C-1), 140.0 (C, C-10), 175.6 (C, C-1'), 212.0 (C, C-11); ES-MS m/z $489.2[\mathrm{M}+\mathrm{H}]^{+}(100)$, 490.3 (80); HRES-MS m/z 489.3687 (calcd for $\mathrm{C}_{29} \mathrm{H}_{49} \mathrm{~N}_{2} \mathrm{O}_{4}, 489.3692$ ). 


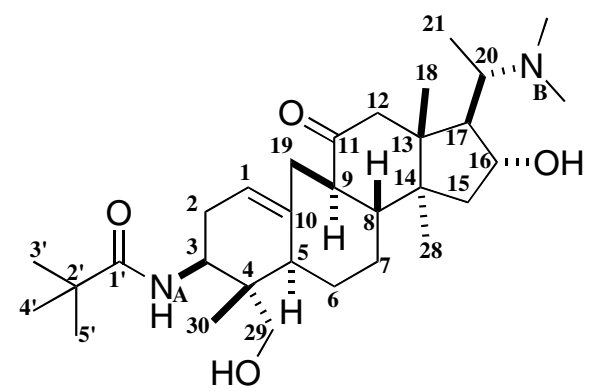

\section{$N$-[(2R,3S,3aR,5aR,9S,10S,10aR,12aR,12bS)-3-[(1S)-1-(dimethylamino)ethyl]-

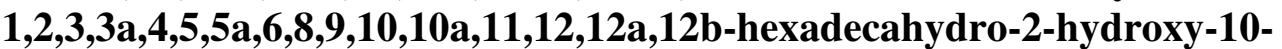 (hydroxymethyl)-3a,10,12b-trimethyl-5-oxobenzo[4,5]cyclohept[1,2-e]inden-9-yl]-2,2- dimethylpropanamide $(8 c)$}

Compound 7c (72 mg, $0.14 \mathrm{mmol}, 1$ equiv) was heated at $250{ }^{\circ} \mathrm{C}$ under $0.03 \mathrm{mmHg}$ in a rotating distillation oven with four bulbs. A product sublimated in 3 hours. The sublimate was composed of $100 \%$ of the expected product 8c. The sublimate was purified by column chromatography on alumina with dichloromethane-methanol (99.7:0.3) as eluent followed by trituration in acetone to afford a colorless powder of 8c $(63.4 \mathrm{mg}, 88 \%): \mathrm{mp} 270{ }^{\circ} \mathrm{C} ;[\alpha]^{22} \mathrm{D}=$ - 3 (c 0.44, $\left.\mathrm{CHCl}_{3}\right)$; IR $\left(\mathrm{CHCl}_{3}\right) \mathrm{v}_{\max }\left(\mathrm{cm}^{-1}\right): 3454,2967,1693,1639,1515,1462,1367,1095$, 1044; ${ }^{1} \mathrm{H}$ NMR $\left(300 \mathrm{MHz}, \mathrm{CDCl}_{3}\right) \delta 0.46(3 \mathrm{H}, \mathrm{s}, \mathrm{H}-30), 0.69(3 \mathrm{H}, \mathrm{s}, \mathrm{H}-18), 0.87(3 \mathrm{H}, \mathrm{d}, J=$ $6.4 \mathrm{~Hz}, \mathrm{H}-21), 1.23$ (9H, s, H-3', H-4', H-5'), 1.27 (3H, s, H-28), 1.27 (1H, m, H-6ß), 1.51 $(1 \mathrm{H}, \mathrm{dd}, J=2.0,13.6 \mathrm{~Hz}, \mathrm{H}-15 \alpha), 1.55(1 \mathrm{H}, \mathrm{bd}, J=10.9 \mathrm{~Hz}, \mathrm{H}-7 \alpha), 1.64(1 \mathrm{H}, \mathrm{m}, \mathrm{H}-8 \beta)$, $1.84(1 \mathrm{H}, \mathrm{m}, \mathrm{H}-6 \alpha), 1.88$ (1H, m, H-7 $\beta), 1.95(1 \mathrm{H}, \mathrm{m}, \mathrm{H}-2 \beta), 1.99(1 \mathrm{H}, \mathrm{m}, \mathrm{H}-15 \beta), 2.03(1 \mathrm{H}$,

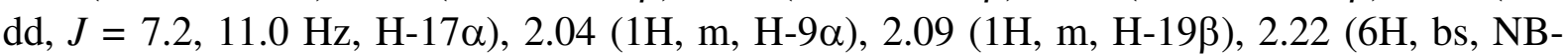
$\left.\mathrm{CH}_{3}\right), 2.28(1 \mathrm{H}, \mathrm{m}, \mathrm{H}-2 \alpha), 2.34(1 \mathrm{H}, \mathrm{d}, J=15.5 \mathrm{~Hz}, \mathrm{H}-12 \beta), 2.58(1 \mathrm{H}, \mathrm{bd}, J=15.5 \mathrm{~Hz}, \mathrm{H}-$ $12 \alpha), 2.60$ (1H, m, H-5a), 2.61 (1H, m, H-20), 3.07 (1H, dd, $J=3.5,10.9 \mathrm{~Hz}, \mathrm{H}-29 \mathrm{a}), 3.35$

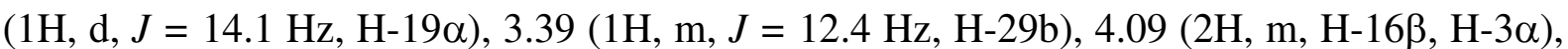
$4.55(1 \mathrm{H}, \mathrm{dd}, J=3.5,10.3 \mathrm{~Hz}, \mathrm{OH}), 5.41(1 \mathrm{H}, \mathrm{d}, J=8.9 \mathrm{~Hz}, \mathrm{NAH}), 5.48(1 \mathrm{H}, \mathrm{m}, \mathrm{H}-1) ;{ }^{13} \mathrm{C}$ NMR (75.5 MHz, $\left.\mathrm{CDCl}_{3}\right) \delta 9.9\left(\mathrm{CH}_{3}, \mathrm{C}-21\right), 10.0\left(\mathrm{CH}_{3}, \mathrm{C}-21, \mathrm{C}-30\right), 17.8\left(\mathrm{CH}_{3}, \mathrm{C}-18\right), 19.0$ $\left(\mathrm{CH}_{3}, \mathrm{C}-28\right), 25.0\left(\mathrm{CH}_{2}, \mathrm{C}-6\right), 27.7\left(\mathrm{CH}_{3}, \mathrm{C}-3\right.$ ', C-4', C-5'), $30.1\left(\mathrm{CH}_{2}, \mathrm{C}-2\right), 33.4\left(\mathrm{CH}_{2}, \mathrm{C}-7\right)$, $37.6\left(\mathrm{CH}_{2}, \mathrm{C}-19\right), 38.8$ (C, C-2'), 42.1 (C, C-4), $42.4\left(\mathrm{CH}_{2}, \mathrm{C}-15\right), 43.4(\mathrm{CH}, \mathrm{C}-5), 46.2$ (C, C-13), 47.0 (C, C-14), $47.6(\mathrm{CH}, \mathrm{C}-3), 50.9$ (CH, C-8), $50.0\left(\mathrm{CH}_{2}, \mathrm{C}-12\right), 50.2(\mathrm{CH}, \mathrm{C}-9)$, $55.3(\mathrm{CH}, \mathrm{C}-17), 62.0(\mathrm{CH}, \mathrm{C}-20), 63.8\left(\mathrm{CH}_{2}, \mathrm{C}-29\right), 78.4(\mathrm{CH}, \mathrm{C}-16), 117.8(\mathrm{CH}, \mathrm{C}-1)$, 140.0 (C, C-10), 180.3 (C, C-1'), 212.0 (C, C-11); ES-MS m/z $517.3[\mathrm{M}+\mathrm{H}]^{+}(100), 518.4$ (60); HRES-MS $m / z 517.3990$ (calcd for $\mathrm{C}_{31} \mathrm{H}_{53} \mathrm{~N}_{2} \mathrm{O}_{4}, 517.4005$ ). 


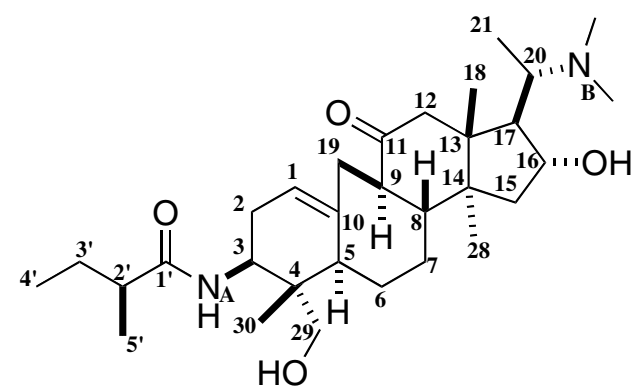

(2S) $-N$-[(2R,3S,3aR,5aR,9S,10S,10aR,12aR,12bS)-3-[(1S)-1-(dimethylamino)ethyl]1,2,3,3a,4,5,5a,6,8,9,10,10a,11,12,12a,12b-hexadecahydro-2-hydroxy-10(hydroxymethyl)-3a,10,12b-trimethyl-5-oxobenzo[4,5]cyclohept[1,2-e]inden-9-yl]-2methylbutanamide $(8 d)$

Compound 7d (84 mg, $0.16 \mathrm{mmol}, 1$ equiv) was heated at $240{ }^{\circ} \mathrm{C}$ under $0.03 \mathrm{mmHg}$ in a rotating distillation oven with four bulbs. A product sublimated in 3 hours. The sublimate was composed of a mixture of the expected product $8 \mathbf{d}$ (85\%) and dihydro-oxazine $9 \mathbf{d}(15 \%)$. The sublimate was purified by column chromatography on alumina with dichloromethanemethanol (99.7:0.3) as eluent followed by trituration in acetone to afford a colorless powder of 8d $(80 \mathrm{mg}, 95 \%): \mathrm{mp} 219{ }^{\circ} \mathrm{C} ;[\alpha]_{\mathrm{D}}^{23}=-14\left(\mathrm{c} 0.6, \mathrm{CHCl}_{3}\right)$; IR $\left(\mathrm{CHCl}_{3}\right) \mathrm{U}_{\max }\left(\mathrm{cm}^{-1}\right): 3433$, 2968, 1693, 1649, 1511, 1462, 1381, 1095, 1043; ${ }^{1} \mathrm{H}$ NMR $\left(300 \mathrm{MHz}, \mathrm{CDCl}_{3}\right) \delta 0.47(3 \mathrm{H}, \mathrm{s}$, H-30), 0.69 (3H, s, H-18), 0.87 (3H, d, $J=6.4 \mathrm{~Hz}, \mathrm{H}-21), 0.95$ (3H, t, $J=7.5 \mathrm{~Hz}, \mathrm{H}-4$ '), 1.15

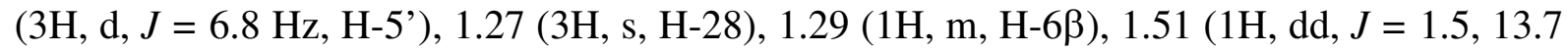

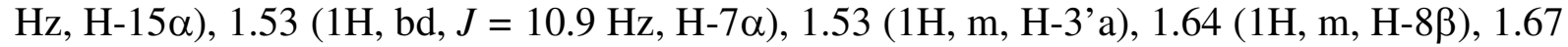

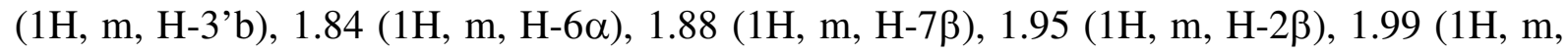
$\mathrm{H}-15 \beta), 2.03(1 \mathrm{H}, \mathrm{dd}, J=7.5,11.0 \mathrm{~Hz}, \mathrm{H}-17 \alpha), 2.04(1 \mathrm{H}, \mathrm{m}, \mathrm{H}-9 \alpha), 2.10(1 \mathrm{H}, \mathrm{m}, \mathrm{H}-19 \beta)$, $2.17\left(1 \mathrm{H}, \mathrm{m}, \mathrm{H}-2\right.$ ') $2.24\left(6 \mathrm{H}, \mathrm{bs}, \mathrm{NB}-\mathrm{CH}_{3}\right), 2.28(1 \mathrm{H}, \mathrm{m}, \mathrm{H}-2 \alpha), 2.33(1 \mathrm{H}, \mathrm{d}, J=15.8 \mathrm{~Hz}, \mathrm{H}-$ $12 \beta), 2.58$ (1H, bd, $J=15.8 \mathrm{~Hz}, \mathrm{H}-12 \alpha), 2.60(1 \mathrm{H}, \mathrm{m}, \mathrm{H}-5 \alpha), 2.60(1 \mathrm{H}, \mathrm{m}, \mathrm{H}-20), 3.07$ (1H, bd, $10.8 \mathrm{~Hz}, \mathrm{H}-29 \mathrm{a}), 3.35$ (1H, d, $J=14.2 \mathrm{~Hz}, \mathrm{H}-19 \alpha), 3.41$ (1H, m, $J=10.0 \mathrm{~Hz}, \mathrm{H}-29 \mathrm{~b})$, $4.08(2 \mathrm{H}, \mathrm{m}, \mathrm{H}-16 \beta, \mathrm{H}-3 \alpha), 4.63(1 \mathrm{H}, \mathrm{m}, \mathrm{OH}), 5.34(1 \mathrm{H}, \mathrm{d}, J=8.9 \mathrm{~Hz}, \mathrm{NAH}), 5.47(1 \mathrm{H}, \mathrm{m}$, $\mathrm{H}-1) ;{ }^{13} \mathrm{C}$ NMR $\left(75.5 \mathrm{MHz}, \mathrm{CDCl}_{3}\right) \delta 10.0\left(\mathrm{CH}_{3}, \mathrm{C}-21, \mathrm{C}-30\right), 12.1\left(\mathrm{CH}_{3}, \mathrm{C}-4\right), 17.6\left(\mathrm{CH}_{3}\right.$, C-5'), $17.8\left(\mathrm{CH}_{3}, \mathrm{C}-18\right), 19.0\left(\mathrm{CH}_{3}, \mathrm{C}-28\right), 25.0\left(\mathrm{CH}_{2}, \mathrm{C}-6\right), 27.5\left(\mathrm{CH}_{2}, \mathrm{C}-3\right.$ ') $) 30.1\left(\mathrm{CH}_{2}, \mathrm{C}-\right.$ 2), $33.4\left(\mathrm{CH}_{2}, \mathrm{C}-7\right), 37.6\left(\mathrm{CH}_{2}, \mathrm{C}-19\right), 42.0$ (C, C-4), $42.4\left(\mathrm{CH}_{2}, \mathrm{C}-15\right), 43.4(\mathrm{CH}, \mathrm{C}-2$ '), 43.5 (CH, C-5), 46.2 (C, C-13), 47.1 (C, C-14), 48.0 (CH, C-3), $50.0(\mathrm{CH}, \mathrm{C}-8), 50.1\left(\mathrm{CH}_{2}, \mathrm{C}-12\right)$, 50.2 (CH, C-9), $55.4(\mathrm{CH}, \mathrm{C}-17), 62.0(\mathrm{CH}, \mathrm{C}-20), 63.8\left(\mathrm{CH}_{2}, \mathrm{C}-29\right), 78.4(\mathrm{CH}, \mathrm{C}-16), 117.9$ (CH, C-1), 140.0 (C, C-10), 178.3 (C, C-1'), 212.0 (C, C-11); ES-MS m/z 517.3 [M+H] ${ }^{+}$ (100), 518.4 (90); HRES-MS $m / z, 517.3980$ (calcd for $\mathrm{C}_{31} \mathrm{H}_{53} \mathrm{~N}_{2} \mathrm{O}_{4}$, 517.4005). 


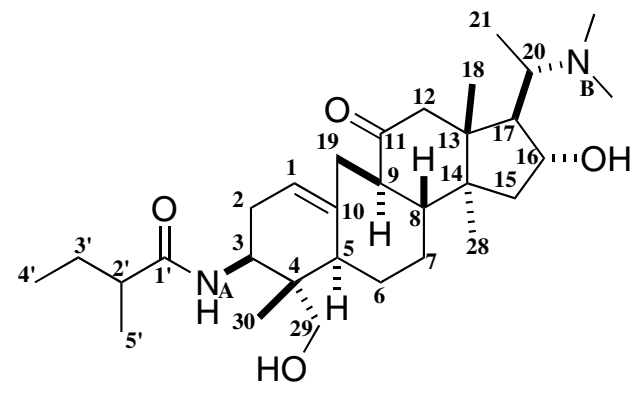

\section{$N$-[(2R,3S,3aR,5aR,9S,10S,10aR,12aR,12bS)-3-[(1S)-1-(dimethylamino)ethyl]- 1,2,3,3a,4,5,5a,6,8,9,10,10a,11,12,12a,12b-hexadecahydro-2-hydroxy-10- (hydroxymethyl)-3a,10,12b-trimethyl-5-oxobenzo[4,5]cyclohept[1,2-e]inden-9-yl]-2- methylbutanamide $(8 e)$}

Compound 7e (40 mg, $0.07 \mathrm{mmol}, 1$ equiv) was heated at $250{ }^{\circ} \mathrm{C}$ under $0.08 \mathrm{mmHg}$ in a rotating distillation oven with four bulbs. A product sublimated in 3 hours. The sublimate was composed of a mixture of the expected product $8 \mathbf{e}(80 \%)$ and dihydro-oxazine $9 \mathbf{e}(20 \%)$. The sublimate was purified by column chromatography on alumina with dichloromethanemethanol (99:1) as eluent followed by trituration in acetone to afford a colorless powder of $8 \mathbf{e}$ (12 mg, 30\%): IR $\left(\mathrm{CHCl}_{3}\right) v_{\max }\left(\mathrm{cm}^{-1}\right): 3433,2935,1693,1650,1511,1462,1377,1096$, 1012; First diastereoisomer ${ }^{1} \mathrm{H}$ NMR $\left(300 \mathrm{MHz}, \mathrm{CDCl}_{3}\right) \delta 0.47(3 \mathrm{H}, \mathrm{s}, \mathrm{H}-30), 0.69(3 \mathrm{H}, \mathrm{s}, \mathrm{H}-$ 18), $0.87(3 \mathrm{H}, \mathrm{d}, J=6.4 \mathrm{~Hz}, \mathrm{H}-21), 0.91\left(3 \mathrm{H}, \mathrm{t}, J=7.3 \mathrm{~Hz}, \mathrm{H}-4^{\prime}\right), 1.16(3 \mathrm{H}, \mathrm{d}, J=7.0 \mathrm{~Hz}, \mathrm{H}-$

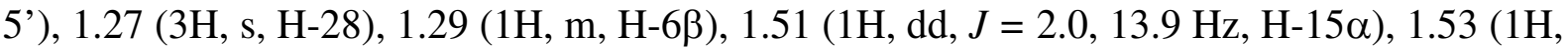

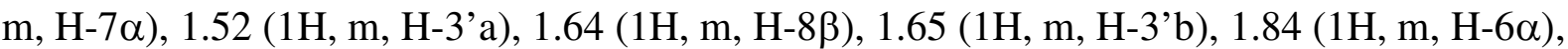

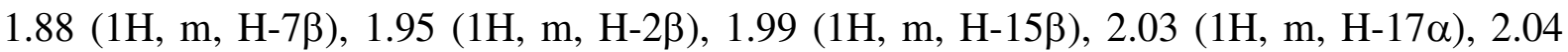

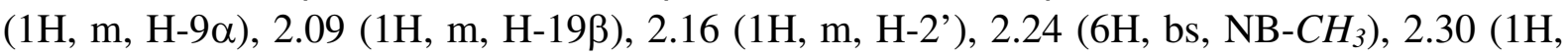
m, H-2 $\alpha), 2.34(1 \mathrm{H}, \mathrm{d}, J=15.6 \mathrm{~Hz}, \mathrm{H}-12 \beta), 2.58(1 \mathrm{H}, \mathrm{bd}, J=15.6 \mathrm{~Hz}, \mathrm{H}-12 \alpha), 2.59(1 \mathrm{H}, \mathrm{m}$, H-5 $), 2.60$ (1H, m, H-20), 3.14 (1H, m, H-29a), 3.35 (1H, d, $J=14.1 \mathrm{~Hz}, \mathrm{H}-19 \alpha), 3.41(1 \mathrm{H}$,

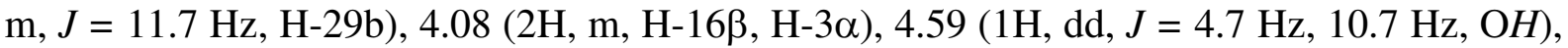
$5.28(1 \mathrm{H}, \mathrm{d}, J=8.9 \mathrm{~Hz}, \mathrm{NAH}), 5.47(1 \mathrm{H}, \mathrm{m}, \mathrm{H}-1) ;{ }^{13} \mathrm{C} \mathrm{NMR}\left(75.5 \mathrm{MHz}, \mathrm{CDCl}_{3}\right) \delta 10.0$ $\left(\mathrm{CH}_{3}, \mathrm{C}-21, \mathrm{C}-30\right), 12.0\left(\mathrm{CH}_{3}, \mathrm{C}-4\right), 17.6\left(\mathrm{CH}_{3}, \mathrm{C}-5\right), 17.8\left(\mathrm{CH}_{3}, \mathrm{C}-18\right), 19.0\left(\mathrm{CH}_{3}, \mathrm{C}-28\right)$, $25.0\left(\mathrm{CH}_{2}, \mathrm{C}-6\right), 27.3\left(\mathrm{CH}_{2}, \mathrm{C}-3\right.$ ') $30.2\left(\mathrm{CH}_{2}, \mathrm{C}-2\right), 33.5\left(\mathrm{CH}_{2}, \mathrm{C}-7\right), 37.6\left(\mathrm{CH}_{2}, \mathrm{C}-19\right), 42.0$ (C, C-4), $42.4\left(\mathrm{CH}_{2}, \mathrm{C}-15\right), 43.4$ (CH, C-8), 43.5 (CH, C-2'), 46.3 (C, C-14), 47.1 (C, C-13), $47.8(\mathrm{CH}, \mathrm{C}-3), 50.0(\mathrm{CH}, \mathrm{C}-8), 50.1\left(\mathrm{CH}_{2}, \mathrm{C}-12\right), 50.2(\mathrm{CH}, \mathrm{C}-9), 55.4(\mathrm{CH}, \mathrm{C}-17), 62.0$ (CH, C-20), 63.8 ( $\left.\mathrm{CH}_{2}, \mathrm{C}-29\right), 78.4(\mathrm{CH}, \mathrm{C}-16), 117.9$ (CH, C-1), 140.0 (C, C-10), 178.3 (C, C-1'), 212.1 (C, C-11); Second diastereoisomer ${ }^{1} \mathrm{H}$ NMR $\left(300 \mathrm{MHz}, \mathrm{CDCl}_{3}\right) \delta 0.47(3 \mathrm{H}, \mathrm{s}, \mathrm{H}-$ 30), 0.69 (3H, s, H-18), 0.87 (3H, d, $J=6.4 \mathrm{~Hz}, \mathrm{H}-21), 0.94$ (3H, t, $J=7.3 \mathrm{~Hz}, \mathrm{H}-4$ '), 1.18 $(3 \mathrm{H}, \mathrm{d}, J=7.0 \mathrm{~Hz}, \mathrm{H}-5$ ') 1.27 (3H, s, H-28), 1.29 (1H, m, H-6ß), 1.51 (1H, dd, $J=2.0,13.9$

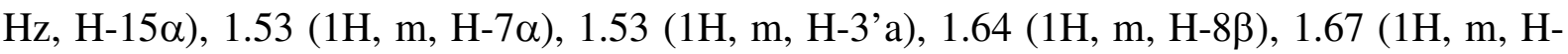

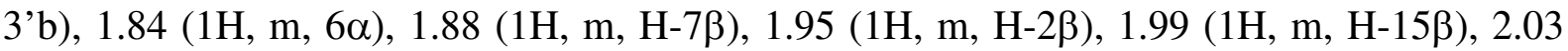

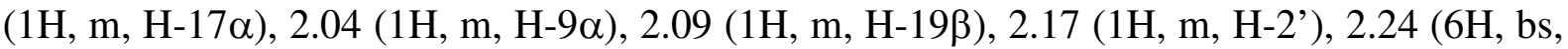
NB- $\left.C_{3}\right), 2.30(1 \mathrm{H}, \mathrm{m}, \mathrm{H}-2 \alpha), 2.34(1 \mathrm{H}, \mathrm{d}, J=15.6 \mathrm{~Hz}, \mathrm{H}-12 \beta), 2.58(1 \mathrm{H}, \mathrm{bd}, J=15.6 \mathrm{~Hz}$,

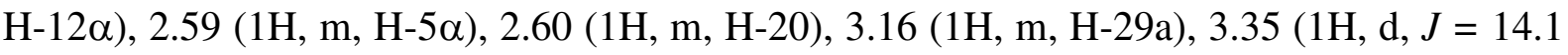

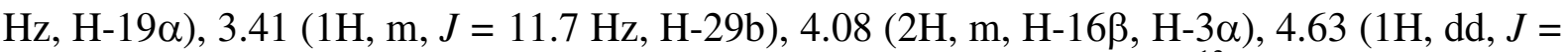
$4.5,10.8 \mathrm{~Hz}, \mathrm{OH}), 5.29(1 \mathrm{H}, \mathrm{d}, J=8.9 \mathrm{~Hz}, \mathrm{NAH}), 5.47(1 \mathrm{H}, \mathrm{m}, \mathrm{H}-1) ;{ }^{13} \mathrm{C}$ NMR $(75.5 \mathrm{MHz}$, $\left.\mathrm{CDCl}_{3}\right) \delta 10.0\left(\mathrm{CH}_{3}, \mathrm{C}-21, \mathrm{C}-30\right), 12.1\left(\mathrm{CH}_{3}, \mathrm{C}-4{ }^{\prime}\right), 17.8\left(\mathrm{CH}_{3}, \mathrm{C}-18\right), 18.0\left(\mathrm{CH}_{3}, \mathrm{C}-5^{\prime}\right), 19.0$ $\left(\mathrm{CH}_{3}, \mathrm{C}-28\right), 25.0\left(\mathrm{CH}_{2}, \mathrm{C}-6\right), 27.5\left(\mathrm{CH}_{2}, \mathrm{C}-3{ }^{\prime}\right), 30.2\left(\mathrm{CH}_{2}, \mathrm{C}-2\right), 33.5\left(\mathrm{CH}_{2}, \mathrm{C}-7\right), 37.6\left(\mathrm{CH}_{2}\right.$, C-19), 42.1 (C, C-4), 42.4 ( $\left.\mathrm{CH}_{2}, \mathrm{C}-15\right), 43.5$ (CH, C-5), 43.5 (CH, C-2'), 46.3 (C, C-14), 47.1 $(\mathrm{C}, \mathrm{C}-13), 47.8(\mathrm{CH}, \mathrm{C}-3), 50.0(\mathrm{CH}, \mathrm{C}-8), 50.1\left(\mathrm{CH}_{2}, \mathrm{C}-12\right), 50.2(\mathrm{CH}, \mathrm{C}-9), 55.4(\mathrm{CH}, \mathrm{C}-$ 
17), $62.0(\mathrm{CH}, \mathrm{C}-20), 63.8\left(\mathrm{CH}_{2}, \mathrm{C}-29\right), 78.4(\mathrm{CH}, \mathrm{C}-16), 117.9(\mathrm{CH}, \mathrm{C}-1), 140.1(\mathrm{C}, \mathrm{C}-10)$, 178.4 (C, C-1'), 212.1 (C, C-11); ES-MS m/z 517.3 [M+H] ${ }^{+}$(100), 518.4 (40), 499.4 (5); HRES-MS $m / z$ 517.4011 (calcd for $\mathrm{C}_{31} \mathrm{H}_{53} \mathrm{~N}_{2} \mathrm{O}_{4}, 517.4005$ ).

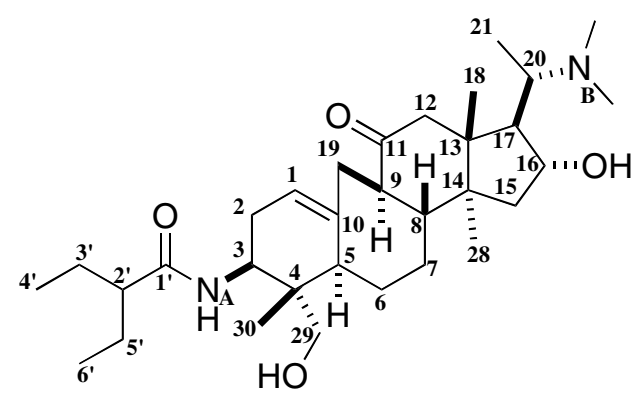

\section{$N-[(2 \mathrm{R}, 3 \mathrm{~S}, 3 \mathrm{aR}, 5 \mathrm{aR}, 9 \mathrm{~S}, 10 \mathrm{~S}, 10 \mathrm{aR}, 12 \mathrm{aR}, 12 \mathrm{bS})-3-[(1 \mathrm{~S})-1-($ dimethylamino)ethyl]- 1,2,3,3a,4,5,5a,6,8,9,10,10a,11,12,12a,12b-hexadecahydro-2-hydroxy-10- (hydroxymethyl)-3a,10,12b-trimethyl-5-oxobenzo[4,5]cyclohept[1,2-e]inden-9-yl]-2- ethylbutanamide $(8 f)$}

Compound 7f (40 mg, $0.08 \mathrm{mmol}, 1$ equiv) was heated at $250{ }^{\circ} \mathrm{C}$ under $0.08 \mathrm{mmHg}$ in a rotating distillation oven with four bulbs. A product sublimated in 3 hours. The sublimate was composed of a mixture of the expected product $\mathbf{8 f}(90 \%)$ and dihydro-oxazine $9 f(10 \%)$. The sublimate was purified by column chromatography on alumina with dichloromethanemethanol (99:1) as eluent followed by trituration in acetone to afford a colorless powder of $\mathbf{8 f}$ (12 mg, 31\%): IR $\left(\mathrm{CHCl}_{3}\right) v_{\max }\left(\mathrm{cm}^{-1}\right): 3433,2966,1693,1649,1510,1461,1381,1096$, $1043 ;{ }^{1} \mathrm{H}$ NMR $\left(300 \mathrm{MHz}, \mathrm{CDCl}_{3}\right) \delta 0.47(3 \mathrm{H}, \mathrm{s}, \mathrm{H}-30), 0.69(3 \mathrm{H}, \mathrm{s}, \mathrm{H}-18), 0.87(3 \mathrm{H}, \mathrm{d}, J=$ $6.6 \mathrm{~Hz}, \mathrm{H}-21), 0.90,0.94$ (6H, 2t, $J=7.3 \mathrm{~Hz}, \mathrm{H}-4$ ', H-6'), 1.27 (3H, s, H-28), 1.29 (1H, m, H$6 \beta), 1.51(1 \mathrm{H}, \mathrm{dd}, J=2.1,13.4 \mathrm{~Hz}, \mathrm{H}-15 \alpha), 1.53$ (1H, m, H-7 $\alpha), 1.53$ (1H, m, H-3'a, H-5'a),

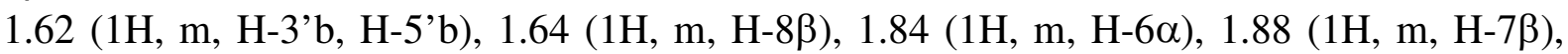

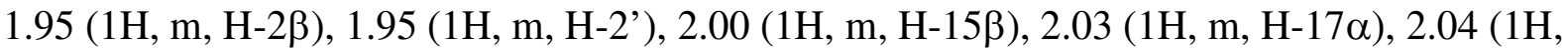

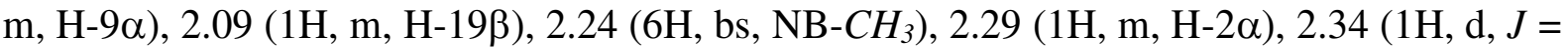

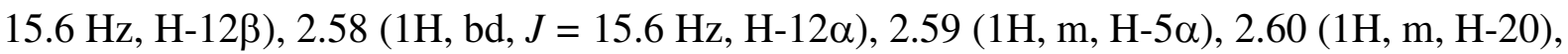
$3.19(1 \mathrm{H}, \mathrm{dd}, J=4.5,12.6 \mathrm{~Hz}, \mathrm{H}-29 \mathrm{a}), 3.35(1 \mathrm{H}, \mathrm{d}, J=14.7 \mathrm{~Hz}, \mathrm{H}-19 \alpha), 3.42(1 \mathrm{H}, \mathrm{dd}, J=$

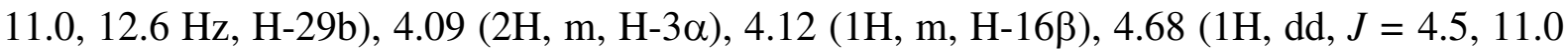
$\mathrm{Hz}, \mathrm{OH}), 5.30(1 \mathrm{H}, \mathrm{d}, J=8.8 \mathrm{~Hz}, \mathrm{NAH}), 5.47(1 \mathrm{H}, \mathrm{m}, \mathrm{H}-1) ;{ }^{13} \mathrm{C} \mathrm{NMR}\left(75.5 \mathrm{MHz}, \mathrm{CDCl}_{3}\right) \delta$ $10.0\left(\mathrm{CH}_{3}, \mathrm{C}-21, \mathrm{C}-30\right), 12.1,12.3\left(\mathrm{CH}_{3}, \mathrm{C}-4\right.$ ', C-6'), $17.8\left(\mathrm{CH}_{3}, \mathrm{C}-18\right), 19.0\left(\mathrm{CH}_{3}, \mathrm{C}-28\right)$, $25.0\left(\mathrm{CH}_{2}, \mathrm{C}-6\right), 25.8,25.9\left(\mathrm{CH}_{2}, \mathrm{C}-3\right.$ ', C-5'), $30.4\left(\mathrm{CH}_{2}, \mathrm{C}-2\right), 33.5\left(\mathrm{CH}_{2}, \mathrm{C}-7\right), 37.6\left(\mathrm{CH}_{2}\right.$, C-19), 41.9 (C, C-4), $42.4\left(\mathrm{CH}_{2}, \mathrm{C}-15\right), 43.4$ (CH, C-5), 46.2 (C, C-14), 47.1 (C, C-13), 48.2 $(\mathrm{CH}, \mathrm{C}-3), 50.0(\mathrm{CH}, \mathrm{C}-8), 50.1\left(\mathrm{CH}_{2}, \mathrm{C}-12\right), 50.2(\mathrm{CH}, \mathrm{C}-9), 51.8(\mathrm{CH}, \mathrm{C}-2$ '), $55.4(\mathrm{CH}, \mathrm{C}-$ 17), $62.0(\mathrm{CH}, \mathrm{C}-20), 63.9\left(\mathrm{CH}_{2}, \mathrm{C}-29\right), 78.4(\mathrm{CH}, \mathrm{C}-16), 117.9(\mathrm{CH}, \mathrm{C}-1), 140.1$ (C, C-10), 177.6 (C, C-1'), 212.0 (C, C-11); ES-MS m/z 531.3 [M+H] ${ }^{+}$(100), 532.4 (50); HRES-MS m/z 531.4173 (calcd for $\mathrm{C}_{32} \mathrm{H}_{55} \mathrm{~N}_{2} \mathrm{O}_{4}, 531.4162$ ). 


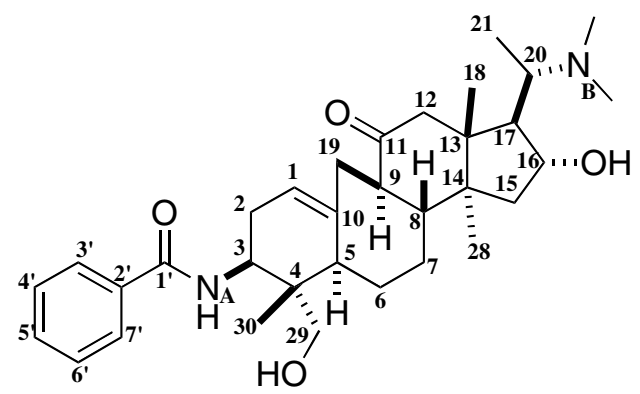

N-[(2R,3S,3aR,5aR,9S,10S,10aR,12aR,12bS)-3-[(1S)-1-(dimethylamino)ethyl]1,2,3,3a,4,5,5a,6,8,9,10,10a,11,12,12a,12b-hexadecahydro-2-hydroxy-10(hydroxymethyl)-3a,10,12b-trimethyl-5-oxobenzo[4,5]cyclohept[1,2-e]inden-9yl]benzamide $(8 g)$

Compound $7 \mathrm{~g}$ (180 mg, $0.33 \mathrm{mmol}, 1$ equiv) was heated at $260{ }^{\circ} \mathrm{C}$ under $0.03 \mathrm{mmHg}$ in a rotating distillation oven with four bulbs. No product sublimated in 3 hours. The residue from the cap was composed of a mixture of the expected product $\mathbf{8 g}(95 \%)$ and dihydro-oxazine $\mathbf{9 g}$ $(5 \%)$. The residue was purified by column chromatography on alumina with dichloromethane-methanol (99.7:0.3) as eluent followed by trituration in acetone to afford a colorless powder of $\mathbf{8 g}(126 \mathrm{mg}, 70 \%)$ : $\mathrm{mp} 289^{\circ} \mathrm{C}$; IR $\left(\mathrm{CHCl}_{3}\right) \mathrm{U}_{\max }\left(\mathrm{cm}^{-1}\right): 3435,2967,1693$, 1644, 1519, 1487, 1452, 1368, 1042; ${ }^{1} \mathrm{H}$ NMR (300 MHz, $\left.\mathrm{CDCl}_{3}\right) \delta 0.54$ (3H, s, H-30), 0.69 (3H, s, H-18), 0.87 (3H, d, J = 6.4 Hz, H-21), 1.27 (3H, s, H-28), 1.29 (1H, m, H-63), 1.51 $(1 \mathrm{H}, \mathrm{dd}, J=2.3,13.6 \mathrm{~Hz}, \mathrm{H}-15 \alpha), 1.55(1 \mathrm{H}, \mathrm{bd}, J=10.7 \mathrm{~Hz}, \mathrm{H}-7 \alpha), 1.64(1 \mathrm{H}, \mathrm{m}, \mathrm{H}-8 \beta)$,

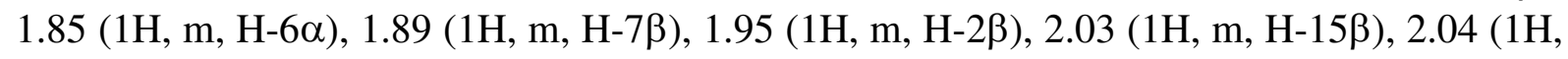

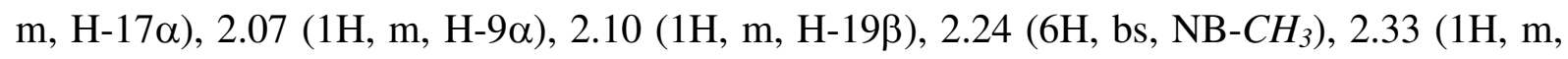
$\mathrm{H}-2 \alpha), 2.33(1 \mathrm{H}, \mathrm{d}, J=15.6 \mathrm{~Hz}, \mathrm{H}-12 \beta), 2.59(1 \mathrm{H}, \mathrm{bd}, J=15.6 \mathrm{~Hz}, \mathrm{H}-12 \alpha), 2.63(1 \mathrm{H}, \mathrm{m}, \mathrm{H}-$

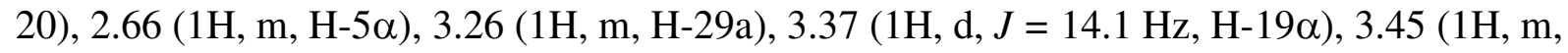
$J=11.9 \mathrm{~Hz}, \mathrm{H}-29 \mathrm{~b}), 4.08(1 \mathrm{H}, \mathrm{m}, \mathrm{H}-16 \beta), 4.30(1 \mathrm{H}, \mathrm{m}, \mathrm{H}-3 \alpha), 4.66(1 \mathrm{H}, \mathrm{m}, \mathrm{OH}), 5.51(1 \mathrm{H}$, $\mathrm{m}, \mathrm{H}-1), 6.08(1 \mathrm{H}, \mathrm{d}, J=9.2 \mathrm{~Hz}, \mathrm{NAH}), 7.46,7.76\left(5 \mathrm{H}, \mathrm{m}, \mathrm{HAr}=3^{\prime}, 4^{\prime}, 6^{\prime}, 7^{\prime}\right) ;{ }^{13} \mathrm{C} \mathrm{NMR}$ (75.5 MHz, $\left.\mathrm{CDCl}_{3}\right) \delta 10.0\left(\mathrm{CH}_{3}, \mathrm{C}-21\right), 10.1\left(\mathrm{CH}_{3}, \mathrm{C}-30\right), 17.7\left(\mathrm{CH}_{3}, \mathrm{C}-18\right), 18.9\left(\mathrm{CH}_{3}, \mathrm{C}-\right.$ 28), $24.9\left(\mathrm{CH}_{2}, \mathrm{C}-7\right), 30.0\left(\mathrm{CH}_{2}, \mathrm{C}-19\right), 33.4\left(\mathrm{CH}_{2}, \mathrm{C}-6\right), 37.5\left(\mathrm{CH}_{2}, \mathrm{C}-2\right), 42.3\left(\mathrm{CH}_{2}, \mathrm{C}-15\right)$, 42.5 (C, C-4), 43.5 (CH, C-5), 46.2 (C, C-14), 47.0 (C, C-13), 48.7 (CH, C-3), 49.9 (CH, C8), $50.0\left(\mathrm{CH}_{2}, \mathrm{C}-12\right), 50.1(\mathrm{CH}, \mathrm{C}-9), 55.3(\mathrm{CH}, \mathrm{C}-17), 62.0(\mathrm{CH}, \mathrm{C}-20), 63.8\left(\mathrm{CH}_{2}, \mathrm{C}-29\right)$, $78.3(\mathrm{CH}, \mathrm{C}-16), 117.8$ (CH, C-1), 127.0, 128.7 (CH, C-Ar = 3',4',6',7'), 131.9 (CH, C-5'), 133.7 (C, C-2'), 140.0 (C, C-10), 169.2 (C, C-1'), 212.0 (C, C-11); ES-MS m/z 537.3 [M+H] ${ }^{+}$ (100), 538.4 (80); HRES-MS $m / z 537.3708$ (calcd for $\mathrm{C}_{33} \mathrm{H}_{49} \mathrm{~N}_{2} \mathrm{O}_{4}, 537.3692$ ). 


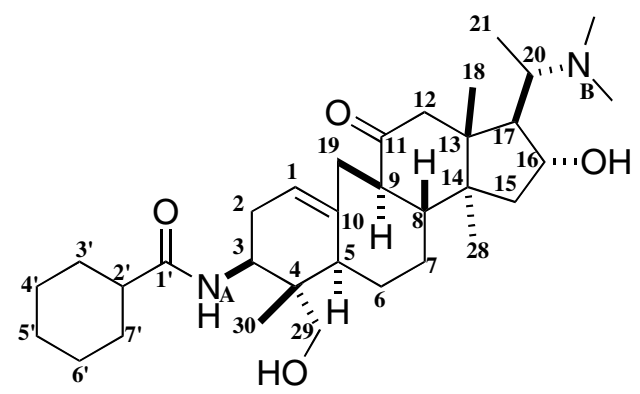

N-[(2R,3S,3aR,5aR,9S,10S,10aR,12aR,12bS)-3-[(1S)-1-(dimethylamino)ethyl]1,2,3,3a,4,5,5a,6,8,9,10,10a,11,12,12a,12b-hexadecahydro-2-hydroxy-10(hydroxymethyl)-3a,10,12b-trimethyl-5-oxobenzo[4,5]cyclohept[1,2-e]inden-9yl]cyclohexanecarboxamide $(8 h)$

Compound $7 \mathrm{~h}$ (85 mg, $0.15 \mathrm{mmol}, 1$ equiv) was heated at $260{ }^{\circ} \mathrm{C}$ under $0.03 \mathrm{mmHg}$ in a rotating distillation oven with four bulbs. No product sublimated in 3 hours. The residue from the cap was composed of a mixture of the expected product $\mathbf{8 h}(83 \%)$ and dihydro-oxazine $\mathbf{9 h}$ $(17 \%)$. The residue was purified by column chromatography on alumina with dichloromethane-methanol (99.7:0.3) as eluent followed by trituration in acetone to afford a colorless powder of $\mathbf{8 h}(80 \mathrm{mg}, 89 \%)$ : mp $285{ }^{\circ} \mathrm{C} ;[\alpha]^{23}{ }_{\mathrm{D}}=-28\left(\mathrm{c} 0.4, \mathrm{CHCl}_{3}\right) ; \mathrm{IR}\left(\mathrm{CHCl}_{3}\right)$ $\mathrm{U}_{\max }\left(\mathrm{cm}^{-1}\right): 3433,2936,1693,1647,1512,1451,1380,1043 ;{ }^{1} \mathrm{H} \mathrm{NMR}\left(300 \mathrm{MHz}, \mathrm{CDCl}_{3}\right) \delta$ 0.46 (3H, s, H-30), 0.69 (3H, s, H-18), 0.87 (3H, d, J = 6.4 Hz, H-21), 1.27 (3H, s, H-28), 1.27 (3H, s, H-28), 1.29 (1H, m, H-63), 1.49-1.52 (5H, m, H-3', H-7', H-5'), 1.51 (1H, dd, J $=2.0,13.5 \mathrm{~Hz}, \mathrm{H}-15 \alpha), 1.55(1 \mathrm{H}, \mathrm{m}, \mathrm{H}-7 \alpha), 1.65(1 \mathrm{H}, \mathrm{m}, \mathrm{H}-8 \beta), 1.84(1 \mathrm{H}, \mathrm{m}, \mathrm{H}-6 \alpha), 1.84$

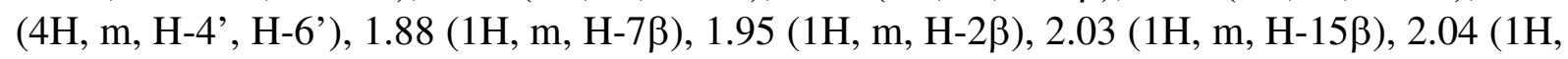

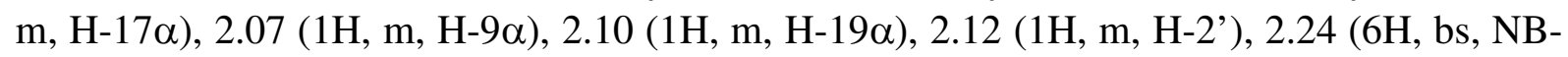
$\left.\mathrm{CH}_{3}\right), 2.31(1 \mathrm{H}, \mathrm{m}, \mathrm{H}-2 \alpha), 2.33(1 \mathrm{H}, \mathrm{d}, J=15.8 \mathrm{~Hz}, \mathrm{H}-12 \beta), 2.58(1 \mathrm{H}, \mathrm{bd}, J=15.8 \mathrm{~Hz}, \mathrm{H}-$ $12 \alpha), 2.59$ (1H, m, H-5 ), 2.60 (1H, m, H-20), 3.11 (1H, dd, $J=4.2,12.6 \mathrm{~Hz}, \mathrm{H}-29 \mathrm{a}), 3.35$

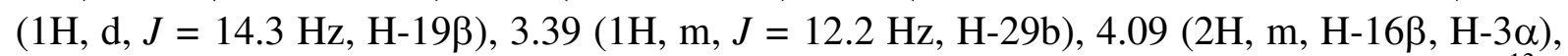
$4.57(1 \mathrm{H}, \mathrm{dd}, J=4.2,10.6 \mathrm{~Hz}, \mathrm{OH}), 5.27(1 \mathrm{H}, \mathrm{d}, J=9.0 \mathrm{~Hz}, \mathrm{NAH}), 5.47(1 \mathrm{H}, \mathrm{m}, \mathrm{H}-1) ;{ }^{13} \mathrm{C}$ NMR (75.5 MHz, $\left.\mathrm{CDCl}_{3}\right) \delta 10.1\left(\mathrm{CH}_{3}, \mathrm{C}-21\right), 10.2\left(\mathrm{CH}_{3}, \mathrm{C}-30\right), 17.9\left(\mathrm{CH}_{3}, \mathrm{C}-18\right), 19.1$ $\left(\mathrm{CH}_{3}, \mathrm{C}-28\right), 25.0\left(\mathrm{CH}_{2}, \mathrm{C}-6\right), 25.7,25.8\left(\mathrm{CH}_{2}, \mathrm{C}-4\right.$ ', C-6'), $29.7\left(\mathrm{CH}_{2}, \mathrm{C}-5\right.$ ' $), 30.2\left(\mathrm{CH}_{2}, \mathrm{C}-\right.$ 2), $30.4\left(\mathrm{CH}_{2}, \mathrm{C}-3\right.$ ', C-7'), $33.6\left(\mathrm{CH}_{2}, \mathrm{C}-7\right), 37.7\left(\mathrm{CH}_{2}, \mathrm{C}-19\right), 42.2(\mathrm{C}, \mathrm{C}-4), 42.6\left(\mathrm{CH}_{2}, \mathrm{C}-\right.$ 15), 43.5 (CH, C-5), 45.8 (CH, C-2'), 46.4 (C, C-14), 47.2 (C, C-13), 47.8 (CH, C-3), 50.1 (CH, C-8), $50.2\left(\mathrm{CH}_{2}, \mathrm{C}-12\right), 50.3(\mathrm{CH}, \mathrm{C}-9), 55.5(\mathrm{CH}, \mathrm{C}-17), 62.2(\mathrm{CH}, \mathrm{C}-20), 63.9\left(\mathrm{CH}_{2}\right.$, C-29), 78.5 (CH, C-16), 118.1 (CH, C-1), 140.2 (C, C-10), 178.1 (C, C-1'), 212.2 (C, C-11); ES-MS $m / z, 543.3[\mathrm{M}+\mathrm{H}]^{+}$(100), 544.4 (70); HRES-MS $m / z 543.4158$ (calcd for $\mathrm{C}_{33} \mathrm{H}_{55} \mathrm{~N}_{2} \mathrm{O}_{4}$, 543.4162). 


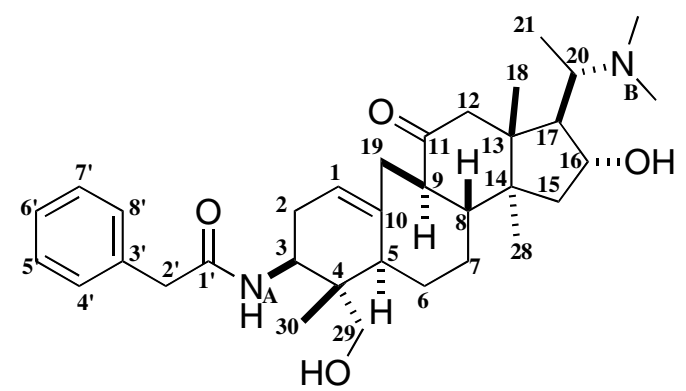

\section{$\mathrm{N}-[(2 \mathrm{R}, 3 \mathrm{~S}, 3 \mathrm{aR}, 5 \mathrm{aR}, 9 \mathrm{~S}, 10 \mathrm{~S}, 10 \mathrm{aR}, 12 \mathrm{aR}, 12 \mathrm{bS})-3-[(1 \mathrm{~S})-1-($ dimethylamino)ethyl]- 1,2,3,3a,4,5,5a,6,8,9,10,10a,11,12,12a,12b-hexadecahydro-2-hydroxy-10- (hydroxymethyl)-3a,10,12b-trimethyl-5-oxobenzo[4,5]cyclohept[1,2-e]inden-9- yl]benzeneacetamide $(8 i)$}

Compound $7 \mathbf{i}$ (102 mg, $0.18 \mathrm{mmol}, 1$ equiv) was heated at $270{ }^{\circ} \mathrm{C}$ under $0.03 \mathrm{mmHg}$ in a rotating distillation oven with four bulbs. No product sublimated in 3 hours. The residue from the cap was composed of a mixture of the expected product $8 \mathbf{i}(74 \%)$ and dihydro-oxazine $9 \mathbf{i}$ (26\%). The residue was purified by column chromatography on alumina with dichloromethane-methanol (99.7:0.3) as eluent followed by trituration in acetone to afford a colorless powder of $\mathbf{8 i}(40.6 \mathrm{mg}, 55 \%): \mathrm{mp} 261{ }^{\circ} \mathrm{C}$; IR $\left(\mathrm{CHCl}_{3}\right) \mathrm{U}_{\max }\left(\mathrm{cm}^{-1}\right): 3411,2938,1693$, $1648,1519,1453,1418,1368,1044 ;{ }^{1} \mathrm{H}$ NMR $\left(300 \mathrm{MHz}, \mathrm{CDCl}_{3}\right) \delta 0.30(3 \mathrm{H}, \mathrm{s}, \mathrm{H}-30), 0.67$ (3H, s, H-18), 0.86 (3H, d, J = 6.4 Hz, H-21), 1.25 (3H, s, H-28), 1.29 (1H, m, H-6ß), 1.51 $(1 \mathrm{H}, \mathrm{dd}, J=3.0,14.0 \mathrm{~Hz}, \mathrm{H}-15 \alpha), 1.55(1 \mathrm{H}, \mathrm{bd}, J=10.7 \mathrm{~Hz}, \mathrm{H}-7 \alpha), 1.64(1 \mathrm{H}, \mathrm{m}, \mathrm{H}-8 \beta)$, $1.80(1 \mathrm{H}, \mathrm{dd}, J=6.4,14.0 \mathrm{~Hz}, \mathrm{H}-6 \alpha), 1.89(1 \mathrm{H}, \mathrm{m}, \mathrm{H}-7 \beta), 1.93(1 \mathrm{H}, \mathrm{m}, \mathrm{H}-2 \beta), 1.99(1 \mathrm{H}, \mathrm{m}$,

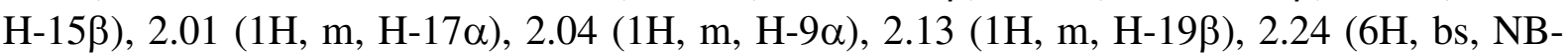
$\left.\mathrm{CH}_{3}\right), 2.32(1 \mathrm{H}, \mathrm{d}, J=15.8 \mathrm{~Hz}, \mathrm{H}-12 \beta), 2.34(1 \mathrm{H}, \mathrm{m}, \mathrm{H}-2 \alpha), 2.57(1 \mathrm{H}, \mathrm{bd}, J=15.8 \mathrm{~Hz}, \mathrm{H}-$ $12 \alpha), 2.57$ (1H, m, H-5a), 2.60 (1H, m, H-20), 3.10 (1H, dd, $J=4.3,12.7$ Hz, H-29a), 3.31 $(1 \mathrm{H}, \mathrm{d}, J=14.5 \mathrm{~Hz}, \mathrm{H}-19 \alpha), 3.39(1 \mathrm{H}, \mathrm{m}, J=11.9 \mathrm{~Hz}, \mathrm{H}-29 \mathrm{~b}), 3.63(2 \mathrm{H}, 2 \mathrm{~d}, J=16.2 \mathrm{~Hz}, \mathrm{H}-$ $\left.2^{\prime}\right), 4.08(2 \mathrm{H}, \mathrm{m}, \mathrm{H}-16 \beta, \mathrm{H}-3 \alpha), 4.44(1 \mathrm{H}, \mathrm{dd}, J=4.3,10.9 \mathrm{~Hz}, \mathrm{OH}), 5.18(1 \mathrm{H}, \mathrm{d}, J=9.0 \mathrm{~Hz}$, NAH $), 5.41(1 \mathrm{H}, \mathrm{m}, \mathrm{H}-1), 7.25-7.40\left(5 \mathrm{H}, \mathrm{m}, \mathrm{HAr}=3^{\prime}, 4^{\prime}, 6^{\prime}, 7^{\prime}\right) ;{ }^{13} \mathrm{C}$ NMR $(75.5 \mathrm{MHz}$, $\left.\mathrm{CDCl}_{3}\right) \delta 9.9\left(\mathrm{CH}_{3}, \mathrm{C}-21\right), 10.1\left(\mathrm{CH}_{3}, \mathrm{C}-30\right), 17.9\left(\mathrm{CH}_{3}, \mathrm{C}-18\right), 19.1\left(\mathrm{CH}_{3}, \mathrm{C}-28\right), 25,1\left(\mathrm{CH}_{2}\right.$, C-6), $29.9\left(\mathrm{CH}_{2}, \mathrm{C}-2\right), 33.6\left(\mathrm{CH}_{2}, \mathrm{C}-7\right), 37.6\left(\mathrm{CH}_{2}, \mathrm{C}-19\right), 42.2(\mathrm{C}, \mathrm{C}-4), 42.6\left(\mathrm{CH}_{2}, \mathrm{C}-15\right)$, $43.5(\mathrm{CH}, \mathrm{C}-5), 43.8\left(\mathrm{CH}_{2}, \mathrm{C}-2\right.$ ') $, 46.4(\mathrm{C}, \mathrm{C}-14), 47.2(\mathrm{C}, \mathrm{C}-13), 48.3(\mathrm{CH}, \mathrm{C}-3), 50.0(\mathrm{CH}$, C-8), $50.2\left(\mathrm{CH}_{2}, \mathrm{C}-12\right), 50.3(\mathrm{CH}, \mathrm{C}-9), 55.5(\mathrm{CH}, \mathrm{C}-17), 62.2(\mathrm{CH}, \mathrm{C}-20), 63.9\left(\mathrm{CH}_{2}, \mathrm{C}-29\right)$, 78.4 (CH, C-16), 117.7 (CH, C-1), 127.7 (CH, C-6'), 129.3 (CH, C-4', C-8', C-5', C-7'), 134.4 (C, C-3'), 139.9 (C, C-10), 172.9 (C, C-1'), 212.0 (C, C-11); ES-MS m/z, 551.3 [M+H] (100), 552.3 (70); HRES-MS m/z 551.3849 (calcd for $\mathrm{C}_{34} \mathrm{H}_{51} \mathrm{~N}_{2} \mathrm{O}_{4}, 551.3817$ ). 


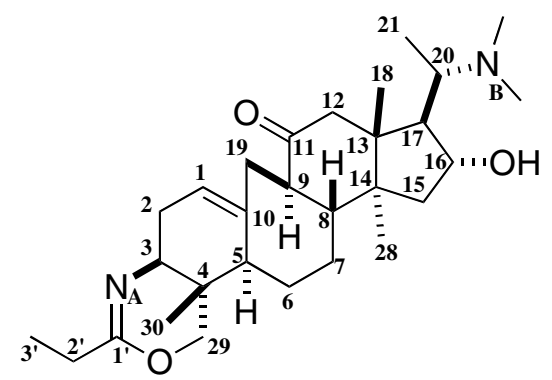

$(4 \mathrm{a} S, 7 \mathrm{a} R, 9 \mathrm{a} R, 10 S, 11 R, 12 \mathrm{a} S, 12 \mathrm{~b} R, 14 \mathrm{~b} S)-10$-[1-(dimethylamino)ethyl]-3-ethyl-11hydroxy-9a,12a,14b-trimethyl-1,4a,5,7,7a,9,9a,10,11,12,12a,12b,13,14,14a,14bhexadecahydro-8H-indeno[ $\left.5^{\prime \prime}, 4^{\prime \prime}: 4^{\prime}, 5^{\prime}\right]$ cyclohepta[1',2':3,4]benzo[1,2- $\left.d\right][1,3]$ oxazin-8-one (9b)

Compound $7 \mathbf{b}$ ( $83 \mathrm{mg}, 0.17 \mathrm{mmol}, 1$ equiv) was heated at $240{ }^{\circ} \mathrm{C}$ under $0.03 \mathrm{mmHg}$ in a rotating distillation oven with four bulbs. A product sublimated in 3 hours. The sublimate was composed of a mixture of the compound $\mathbf{8 b}(64 \%)$ and the expected dihydro-oxazine $\mathbf{9 b}$ (36\%). To a solution of the sublimate in $2 \mathrm{~mL}$ of dichloromethane was added $25 \%$ tetraethylammonium hydroxide $(500 \mathrm{mg}, 0.85 \mathrm{mmol}, 5$ equiv) solution in methanol. The solvent was evaporated under reduced pressure. The dark red residue was heated at $240{ }^{\circ} \mathrm{C}$ under $0.03 \mathrm{mmHg}$ in a rotating distillation oven with four bulbs. A product sublimated in 3 hours in the form of cristals to afford a pale yellow powder of $9 \mathbf{b}(75 \mathrm{mg}, 94 \%): \mathrm{mp} 282{ }^{\circ} \mathrm{C}$; $[\alpha]_{D}^{22}=+76\left(\mathrm{c} \mathrm{1.4}, \mathrm{CHCl}_{3}\right) ; \mathrm{IR}\left(\mathrm{CHCl}_{3}\right) \cup_{\max }\left(\mathrm{cm}^{-1}\right): 3363,2978,1694,1667,1462,1390$, $1040 ;{ }^{1} \mathrm{H}$ NMR $\left(300 \mathrm{MHz}, \mathrm{CDCl}_{3}\right) \delta 0.70(3 \mathrm{H}, \mathrm{s}, \mathrm{H}-18), 0.77(3 \mathrm{H}, \mathrm{s}, \mathrm{H}-30), 0.87(3 \mathrm{H}, \mathrm{d}, J=$ $6.6 \mathrm{~Hz}, \mathrm{H}-21), 1.13$ (3H, d, J = 7.5 Hz, H-3'), 1.24 (3H, s, H-28), 1.29 (1H, m, H-6ß), 1.34 $(1 \mathrm{H}, \mathrm{m}, \mathrm{H}-7 \alpha), 1.51(1 \mathrm{H}, \mathrm{dd}, J=2.3,13.8 \mathrm{~Hz}, \mathrm{H}-15 \alpha), 1.62(1 \mathrm{H}, \mathrm{m}, \mathrm{H}-6 \alpha), 1.66(1 \mathrm{H}, \mathrm{m}, \mathrm{H}-$

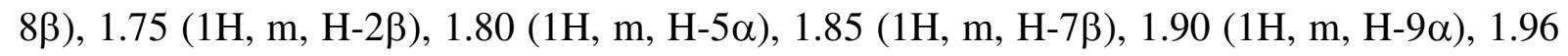

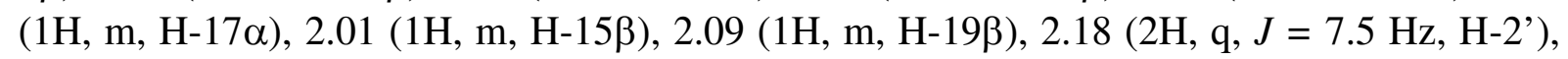
$2.24\left(6 \mathrm{H}, \mathrm{bs}, \mathrm{NB}-\mathrm{CH}_{3}\right), 2.34(1 \mathrm{H}, \mathrm{d}, J=16.0 \mathrm{~Hz}, \mathrm{H}-12 \beta), 2.30(1 \mathrm{H}, \mathrm{m}, \mathrm{H}-2 \alpha), 2.56(1 \mathrm{H}, \mathrm{bd}$, $J=16.0 \mathrm{~Hz}, \mathrm{H}-12 \alpha), 2.59(1 \mathrm{H}, \mathrm{dd}, J=6.6,10.8 \mathrm{~Hz}, \mathrm{H}-20), 3.25(1 \mathrm{H}, \mathrm{dd}, J=5.8,11.5 \mathrm{~Hz}$,

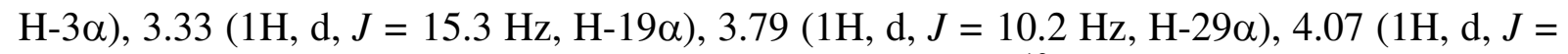
$10.2 \mathrm{~Hz}, \mathrm{H}-29 \beta), 4.09(1 \mathrm{H}, \mathrm{m}, \mathrm{H}-16 \beta), 5.60(1 \mathrm{H}, \mathrm{m}, \mathrm{H}-1) ;{ }^{13} \mathrm{C} \mathrm{NMR}\left(75.5 \mathrm{MHz}, \mathrm{CDCl}_{3}\right) \delta$ $9.8\left(\mathrm{CH}_{3}, \mathrm{C}-30\right), 10.0\left(\mathrm{CH}_{3}, \mathrm{C}-21\right), 11.0\left(\mathrm{CH}_{3}, \mathrm{C}-3\right), 17.8\left(\mathrm{CH}_{3}, \mathrm{C}-18\right), 18.8\left(\mathrm{CH}_{3}, \mathrm{C}-28\right)$, $24.6\left(\mathrm{CH}_{2}, \mathrm{C}-6\right), 28.3\left(\mathrm{CH}_{2}, \mathrm{C}-2\right)$ ') $30.7\left(\mathrm{CH}_{2}, \mathrm{C}-2\right), 33.1$ (C, C-4), $33.5\left(\mathrm{CH}_{2}, \mathrm{C}-7\right), 37.3$ $\left(\mathrm{CH}_{2}, \mathrm{C}-19\right), 42,4\left(\mathrm{CH}_{2}, \mathrm{C}-15\right), 46.1$ (C, C-14), 47.0 (C, C-13), 49.4 (CH, C-5), 49.7 (CH, C8), $50.0\left(\mathrm{CH}_{2}, \mathrm{C}-12\right), 50.2(\mathrm{CH}, \mathrm{C}-9), 55.4(\mathrm{CH}, \mathrm{C}-17), 55.7(\mathrm{CH}, \mathrm{C}-3), 62.0(\mathrm{CH}, \mathrm{C}-20), 74.8$ ( $\left.\mathrm{CH}_{2}, \mathrm{C}-29\right), 78.3$ (CH, C-16), 121.7 (CH, C-1), 137.2 (C, C-10), 160.1 (C, C-1'), 211.7 (C, C-11); ES-MS m/z $471.3[\mathrm{M}+\mathrm{H}]^{+}$(100), 472.4 (70), $236.2[\mathrm{M}+2 \mathrm{H}]^{+} / 2$ (90), 236.7 (50); HRES-MS $m / z 471.3577$ (calcd for $\mathrm{C}_{29} \mathrm{H}_{47} \mathrm{~N}_{2} \mathrm{O}_{3}, 471.3587$ ). 


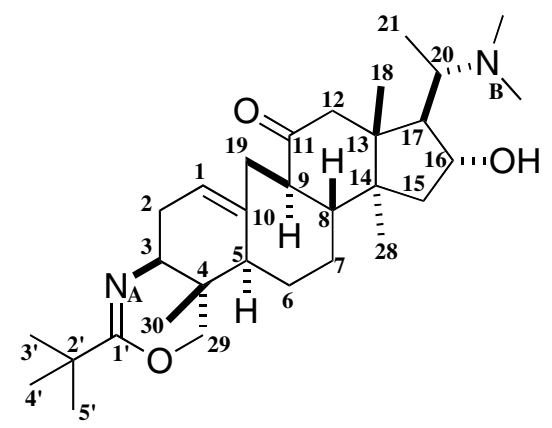

$(4 \mathrm{a} S, 7 \mathrm{a} R, 9 \mathrm{a} R, 10 S, 11 R, 12 \mathrm{a} S, 12 \mathrm{~b} R, 14 \mathrm{~b} S)-3-t e r t-b u t y l-10-[1-(d i m e t h y l a m i n o) e t h y l]-11$ -hydroxy-9a,12a,14b-trimethyl-1,4a,5,7,7a,9,9a,10,11,12,12a,12b,13,14,14a,14b-he xadecahydro-8H-indeno[5", $\left.4^{\prime \prime}: 4^{\prime}, 5^{\prime}\right]$ cyclohepta[1',2':3,4]benzo[1,2-d][1,3]oxazin -8-one $(9 c)$

Compound 7c (73 mg, $0.14 \mathrm{mmol}, 1$ equiv) was heated at $250{ }^{\circ} \mathrm{C}$ under $0.03 \mathrm{mmHg}$ in a rotating distillation oven with four bulbs. No product sublimated in 3 hours. The residue from the cap was composed of the compound 8c (100\%). To a solution of the residue in $2 \mathrm{~mL}$ of dichloromethane was added $25 \%$ tetraethylammonium hydroxide (416 $\mathrm{mg}, 0.7 \mathrm{mmol}, 5$ equiv) solution in methanol. The solvent was evaporated under reduced pressure. The dark red residue was heated at $250{ }^{\circ} \mathrm{C}$ under $0.03 \mathrm{mmHg}$ in a rotating distillation oven with four bulbs. A product sublimated in 3 hours to afford an amorphous yellow powder of 9c $(60.4 \mathrm{mg}$, 85\%): IR $\left(\mathrm{CHCl}_{3}\right) \cup_{\max }\left(\mathrm{cm}^{-1}\right): 3373,1693,1655,1461,1366,1015 ;{ }^{1} \mathrm{H}$ NMR $(400 \mathrm{MHz}$, $\left.\mathrm{CDCl}_{3}\right) \delta 0.69(3 \mathrm{H}, \mathrm{s}, \mathrm{H}-18), 0.72(3 \mathrm{H}, \mathrm{s}, \mathrm{H}-30), 0.87(3 \mathrm{H}, \mathrm{d}, J=6.4 \mathrm{~Hz}, \mathrm{H}-21), 1.14(9 \mathrm{H}, \mathrm{s}$,

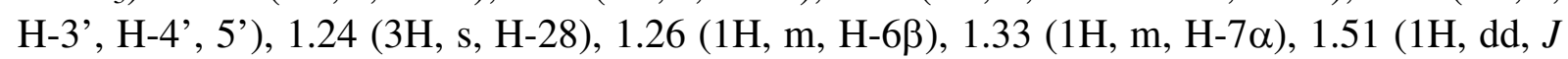

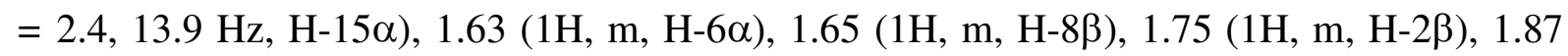

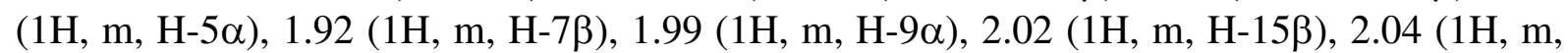
$\mathrm{H}-17 \alpha), 2.08$ (1H, m, H-19ß), 2.24 (6H, bs, NB-CH $), 2.34$ (1H, d, $J=16.3 \mathrm{~Hz}, \mathrm{H}-12 \beta), 2.35$ $(1 \mathrm{H}, \mathrm{m}, \mathrm{H}-2 \alpha), 2.56(1 \mathrm{H}, \mathrm{bd}, J=16.3 \mathrm{~Hz}, \mathrm{H}-12 \alpha), 2.60(1 \mathrm{H}, \mathrm{dd}, J=6.4,11.0 \mathrm{~Hz}, \mathrm{H}-20)$, $3.23(1 \mathrm{H}, \mathrm{dd}, J=6.0,11.5 \mathrm{~Hz}, \mathrm{H}-3 \alpha), 3.34(1 \mathrm{H}, \mathrm{d}, J=15.4 \mathrm{~Hz}, \mathrm{H}-19 \alpha), 3.73(1 \mathrm{H}, \mathrm{d}, J=$ $10.3 \mathrm{~Hz}, \mathrm{H}-29 \alpha), 4.06$ (1H, d, $J=10.3 \mathrm{~Hz}, \mathrm{H}-29 \beta), 4.08$ (1H, m, H-16ß), 5.60 (1H, m, H-1); ${ }^{13} \mathrm{C}$ NMR $\left(100 \mathrm{MHz}, \mathrm{CDCl}_{3}\right) \delta 9.57\left(\mathrm{CH}_{3}, \mathrm{C}-30\right), 10.01\left(\mathrm{CH}_{3}, \mathrm{C}-21\right), 17.82\left(\mathrm{CH}_{3}, \mathrm{C}-18\right)$, $18.85\left(\mathrm{CH}_{3}, \mathrm{C}-28\right), 24.65\left(\mathrm{CH}_{2}, \mathrm{C}-6\right), 27.89\left(\mathrm{CH}_{3}, \mathrm{C}-3\right.$ ', C-4', C-5'), $30.98\left(\mathrm{CH}_{2}, \mathrm{C}-2\right), 33,14$ (C, C-4), $33.54\left(\mathrm{CH}_{2}, \mathrm{C}-7\right), 37.37\left(\mathrm{CH}_{2}, \mathrm{C}-19\right), 38.83$ (C, C-2'), $42.50\left(\mathrm{CH}_{2}, \mathrm{C}-15\right), 46.18(\mathrm{C}$, $\mathrm{C}-14), 47.04$ (C, C-13), 49.52 (CH, C-5), 49.77 (CH, C-8), $50.04\left(\mathrm{CH}_{2}, \mathrm{C}-12\right), 50.34(\mathrm{CH}, \mathrm{C}-$ 9), $55.50(\mathrm{CH}, \mathrm{C}-17), 55.93(\mathrm{CH}, \mathrm{C}-3), 62.05(\mathrm{CH}, \mathrm{C}-20), 74.69\left(\mathrm{CH}_{2}, \mathrm{C}-29\right), 78.36(\mathrm{CH}, \mathrm{C}-$ 16), 121.87 (CH, C-1), 137.15 (C, C-10), 161.90 (C, C-1'), 211.61 (C, C-11); ES-MS m/z $499.4[\mathrm{M}+\mathrm{H}]^{+}$(100), 500.4 (40); HRES-MS $m / z 499.3868$ (calcd for $\mathrm{C}_{31} \mathrm{H}_{51} \mathrm{~N}_{2} \mathrm{O}_{3}, 499.3900$ ). 


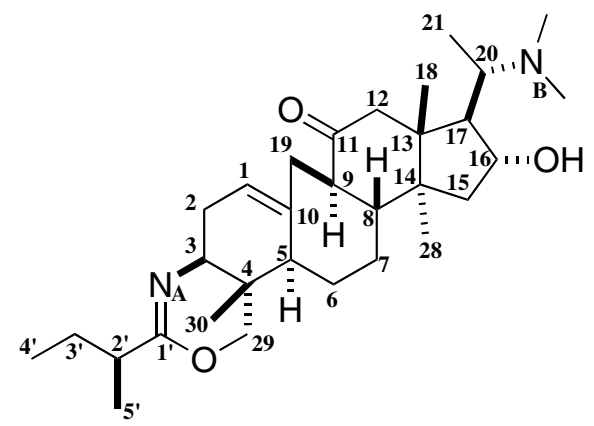

(4a $S, 7 \mathrm{a} R, 9 \mathrm{a} R, 10 S, 11 R, 12 \mathrm{a} S, 12 \mathrm{~b} R, 14 \mathrm{~b} S)$-10-[1-(dimethylamino)ethyl]-11-hydroxy-9a,1 2a,14b-trimethyl-3-[(1S)-1-methylpropyl]-1,4a,5,7,7a,9,9a,10,11,12,12a,12b,13,14 ,14a,14b-hexadecahydro-8H-indeno $\left[5^{\prime \prime}, 4^{\prime \prime}: 4^{\prime}, 5^{\prime}\right]$ cyclohepta[1',2':3,4]benzo[1,2-d] [1,3]oxazin-8-one (9d)

Compound 7d (63 mg, $0.12 \mathrm{mmol}, 1$ equiv) was heated at $253{ }^{\circ} \mathrm{C}$ under $0.04 \mathrm{mmHg}$ in a rotating distillation oven with four bulbs. A product sublimated in 3 hours. The sublimate was composed of a mixture of the compound 8d (85\%) and the expected dihydro-oxazine 9d $(15 \%)$. To a solution of the residue from the cap and the sublimate in $2 \mathrm{~mL}$ of dichloromethane was added $25 \%$ tetraethylammonium hydroxide $(364 \mathrm{mg}, 0.6 \mathrm{mmol}, 5$ equiv) solution in methanol. The solvent was evaporated under reduced pressure. The dark red residue was heated at $253{ }^{\circ} \mathrm{C}$ under $0.04 \mathrm{mmHg}$ in a rotating distillation oven with four bulbs. A product sublimated in 3 hours to afford an sublimated pale yellow powder of $9 d(49 \mathrm{mg}$, 80\%): IR $\left(\mathrm{CHCl}_{3}\right) \cup_{\max }\left(\mathrm{cm}^{-1}\right): 3433,2969,1694,1659,1462,1378,1080,1040 ;{ }^{1} \mathrm{H}$ NMR $\left(400 \mathrm{MHz}, \mathrm{CDCl}_{3}\right) \delta 0.70(3 \mathrm{H}, \mathrm{s}, \mathrm{H}-18), 0.78(3 \mathrm{H}, \mathrm{s}, \mathrm{H}-30), 0.87(3 \mathrm{H}, \mathrm{d}, J=6.0 \mathrm{~Hz}, \mathrm{H}-21)$, $0.90\left(3 \mathrm{H}, \mathrm{t}, J=7.5 \mathrm{~Hz}, \mathrm{H}-4^{\prime}\right), 1.10(3 \mathrm{H}, \mathrm{d}, J=6.8 \mathrm{~Hz}, \mathrm{H}-5$ '), $1.24(3 \mathrm{H}, \mathrm{s}, \mathrm{H}-28), 1.30(1 \mathrm{H}$,

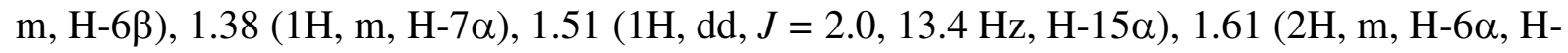

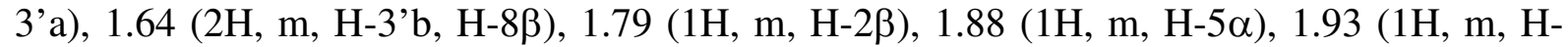

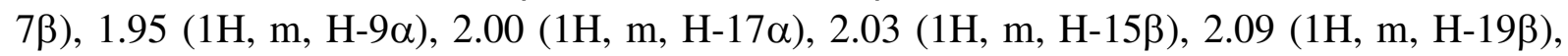
$2.18\left(1 \mathrm{H}, \mathrm{m}, \mathrm{H}-2\right.$ ') 2.25 (6H, bs, NB-CH $\mathrm{CH}_{3}, 2.35$ (1H, d, $\left.J=16.4 \mathrm{~Hz}, \mathrm{H}-12 \beta\right), 2.32(1 \mathrm{H}, \mathrm{m}$, $\mathrm{H}-2 \alpha), 2.56(1 \mathrm{H}, \mathrm{bd}, J=16.4 \mathrm{~Hz}, \mathrm{H}-12 \alpha), 2.61(1 \mathrm{H}, \mathrm{dd}, J=6.0,11.0 \mathrm{~Hz}, \mathrm{H}-20), 3.26(1 \mathrm{H}$, $\mathrm{dd}, J=6.0,12.0 \mathrm{~Hz}, \mathrm{H}-3 \alpha), 3.34(1 \mathrm{H}, \mathrm{d}, J=16.4 \mathrm{~Hz}, \mathrm{H}-19 \alpha), 3.77(1 \mathrm{H}, \mathrm{d}, J=10.4 \mathrm{~Hz}, \mathrm{H}-$ $29 \alpha), 4.08$ (1H, d, $J=10.4 \mathrm{~Hz}, \mathrm{H}-29 \beta), 4.09$ (1H, m, H-16ß), 5.60 (1H, m, H-1); ${ }^{13} \mathrm{C} \mathrm{NMR}$ $\left(100 \mathrm{MHz}, \mathrm{CDCl}_{3}\right) \delta 9.68\left(\mathrm{CH}_{3}, \mathrm{C}-30\right), 9.95\left(\mathrm{CH}_{3}, \mathrm{C}-21\right), 12.02\left(\mathrm{CH}_{3}, \mathrm{C}-4\right), 17.68\left(\mathrm{CH}_{3}, \mathrm{C}-\right.$ 5'), $17.79\left(\mathrm{CH}_{3}, \mathrm{C}-18\right), 18.81\left(\mathrm{CH}_{3}, \mathrm{C}-28\right), 24.60\left(\mathrm{CH}_{2}, \mathrm{C}-6\right), 27.21\left(\mathrm{CH}_{2}, \mathrm{C}-3\right), 30.76\left(\mathrm{CH}_{2}\right.$, C-2), 33.16 (C, C-4), $33.49\left(\mathrm{CH}_{2}, \mathrm{C}-7\right), 37.28\left(\mathrm{CH}_{2}, \mathrm{C}-19\right), 41.56\left(\mathrm{CH}, \mathrm{C}-2\right.$ '), $42.44\left(\mathrm{CH}_{2}, \mathrm{C}-\right.$ 15), 46.15 (C, C-14), 46.98 (C, C-13), 49.53 (CH, C-5), 49.68 (CH, C-8), $49.99\left(\mathrm{CH}_{2}, \mathrm{C}-12\right)$, $50.26(\mathrm{CH}, \mathrm{C}-9), 55.45(\mathrm{CH}, \mathrm{C}-17), 55.60(\mathrm{CH}, \mathrm{C}-3), 62.00(\mathrm{CH}, \mathrm{C}-20), 74.71\left(\mathrm{CH}_{2}, \mathrm{C}-29\right)$, 78.28 (CH, C-16), 121.87 (CH, C-1), 137.15 (C, C-10), 161.90 (C, C-1'), 211.61 (C, C-11); ES-MS m/z $499.4[\mathrm{M}+\mathrm{H}]^{+}$(100), 500.4 (50), $250.2[\mathrm{M}+\mathrm{H}]^{+} / 2$ (70), 250.7 (45); HRES-MS $\mathrm{m} / \mathrm{z}$ 499.3917 (calcd for $\mathrm{C}_{31} \mathrm{H}_{51} \mathrm{~N}_{2} \mathrm{O}_{3}, 499.3900$ ). 


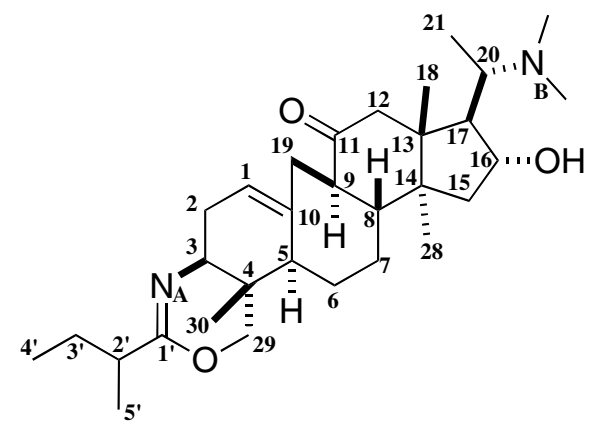

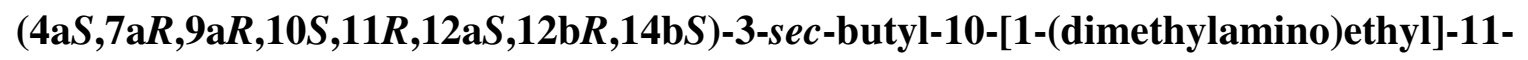
hydroxy-9a,12a,14b-trimethyl-1,4a,5,7,7a,9,9a,10,11,12,12a,12b,13,14,14a,14b-hex adecahydro-8H-indeno[ $\left.5^{\prime \prime}, 4^{\prime \prime}: 4^{\prime}, 5^{\prime}\right]$ cyclohepta[1',2' :3,4]benzo[1,2-d][1,3] oxazin8-one $(9 e)$

Compound 7e (105 mg, $0.2 \mathrm{mmol}, 1$ equiv) was heated at $257{ }^{\circ} \mathrm{C}$ under $0.1 \mathrm{mmHg}$ in a rotating distillation oven with four bulbs. A product sublimated in 3 hours. The sublimate was composed of a mixture of the compound 8e (85\%) and the expected dihydro-oxazine 9e $(15 \%)$. To a solution of the residue from the cap and the sublimate in $2 \mathrm{~mL}$ of dichloromethane was added $25 \%$ tetraethylammonium hydroxide solution $(597 \mathrm{mg}, 1 \mathrm{mmol}, 5$ equiv) in methanol. The solvent was evaporated under reduced pressure. The dark red residue was heated at $260{ }^{\circ} \mathrm{C}$ under $0.1 \mathrm{mmHg}$ in a rotating distillation oven with four bulbs. A product sublimated in 5 hours in three times to afford an amorphous pale yellow powder of $9 \mathbf{e}$ (30 mg, 30\%): IR $\left(\mathrm{CHCl}_{3}\right) \cup_{\max }\left(\mathrm{cm}^{-1}\right): 3461,1695,1651,1461,1375,1097$; First diastereoisomer ${ }^{1} \mathrm{H}$ NMR (400 MHz, $\left.\mathrm{CDCl}_{3}\right) \delta 0.70(3 \mathrm{H}, \mathrm{s}, \mathrm{H}-18), 0.77(3 \mathrm{H}, \mathrm{s}, \mathrm{H}-30), 0.87$ $(3 \mathrm{H}, \mathrm{d}, J=6.0 \mathrm{~Hz}, \mathrm{H}-21), 0.90$ (3H, t, $J=7.5 \mathrm{~Hz}, \mathrm{H}-4$ '), 1.09 (3H, d, H-5'), 1.24 (3H, s, H28), $1.30(1 \mathrm{H}, \mathrm{m}, \mathrm{H}-6 \beta), 1.38(1 \mathrm{H}, \mathrm{m}, \mathrm{H}-7 \alpha), 1.51(1 \mathrm{H}, \mathrm{dd}, J=2.0,13.4 \mathrm{~Hz}, \mathrm{H}-15 \alpha), 1.57-$

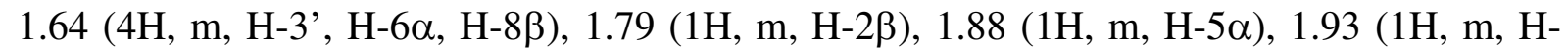

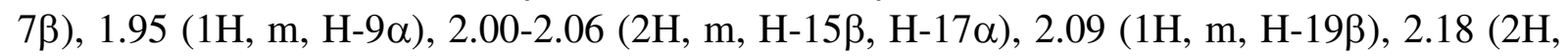
q, $J=7.5 \mathrm{~Hz}, \mathrm{H}-2$ '), 2.25 (6H, bs, NB- $\left.\mathrm{CH}_{3}\right), 2.35(1 \mathrm{H}, \mathrm{d}, J=16.4 \mathrm{~Hz}, \mathrm{H}-12 \beta), 2.35(1 \mathrm{H}, \mathrm{m}$, $\mathrm{H}-2 \alpha), 2.56(1 \mathrm{H}, \mathrm{bd}, J=16.4 \mathrm{~Hz}, \mathrm{H}-12 \alpha), 2.61(1 \mathrm{H}, \mathrm{m}, \mathrm{H}-20), 3.26(1 \mathrm{H}, \mathrm{dd}, J=6.0,12.0$ $\mathrm{Hz}, \mathrm{H}-3 \alpha), 3.35$ (1H, d, $J=16.4 \mathrm{~Hz}, \mathrm{H}-19 \alpha), 3.77$ (1H, d, $J=10.4 \mathrm{~Hz}, \mathrm{H}-29 \alpha), 4.07$ (1H, d, $J=10.4 \mathrm{~Hz}, \mathrm{H}-29 \beta), 4.09(1 \mathrm{H}, \mathrm{m}, \mathrm{H}-16 \beta), 5.60(1 \mathrm{H}, \mathrm{m}, \mathrm{H}-1) ;{ }^{13} \mathrm{C} \mathrm{NMR}\left(100 \mathrm{MHz}, \mathrm{CDCl}_{3}\right) \delta$ $9.68\left(\mathrm{CH}_{3}, \mathrm{C}-30\right), 9.95\left(\mathrm{CH}_{3}, \mathrm{C}-21\right), 12.02\left(\mathrm{CH}_{3}\right.$, bs, C-4'), $17.68\left(\mathrm{CH}_{3}\right.$, bs, C-5'), 17.79 $\left(\mathrm{CH}_{3}, \mathrm{C}-18\right), 18.81\left(\mathrm{CH}_{3}, \mathrm{C}-28\right), 24.60\left(\mathrm{CH}_{2}, \mathrm{C}-6\right), 27.11\left(\mathrm{CH}_{2}, \mathrm{C}-3\right.$ '), $30.76\left(\mathrm{CH}_{2}, \mathrm{C}-2\right)$, 33.16 (C, C-4), $33.49\left(\mathrm{CH}_{2}, \mathrm{C}-7\right), 37.28\left(\mathrm{CH}_{2}, \mathrm{C}-19\right), 41.56\left(\mathrm{CH}, \mathrm{C}-2{ }^{\prime}\right), 42.44\left(\mathrm{CH}_{2}, \mathrm{C}-15\right)$, 46.15 (C, C-14), 46.98 (C, C-13), 49.53 (CH, C-5), 49.68 (CH, C-8), $49.99\left(\mathrm{CH}_{2}, \mathrm{C}-12\right)$, 50.26 (CH, C-9), 55.45 (CH, C-17), $55.60(\mathrm{CH}, \mathrm{C}-3), 62.00(\mathrm{CH}, \mathrm{C}-20), 74.71\left(\mathrm{CH}_{2}, \mathrm{C}-29\right)$, 78.28 (CH, C-16), 121.87 (CH, C-1), 137.15 (C, C-10), 161.90 (C, C-1'), 211.61 (C, C-11); Second diastereoisomer ${ }^{1} \mathrm{H}$ NMR $\left(400 \mathrm{MHz}, \mathrm{CDCl}_{3}\right) \delta 0.70(3 \mathrm{H}, \mathrm{s}, \mathrm{H}-18), 0.78(3 \mathrm{H}, \mathrm{s}, \mathrm{H}-30)$, $0.87(3 \mathrm{H}, \mathrm{d}, J=6.0 \mathrm{~Hz}, \mathrm{H}-21), 0.92(3 \mathrm{H}, \mathrm{t}, J=7.5 \mathrm{~Hz}, \mathrm{H}-4$ ') 1.11 (3H, d, H-5'), 1.24 (3H, s,

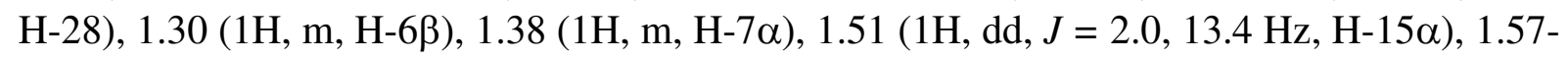

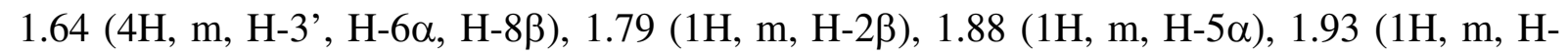

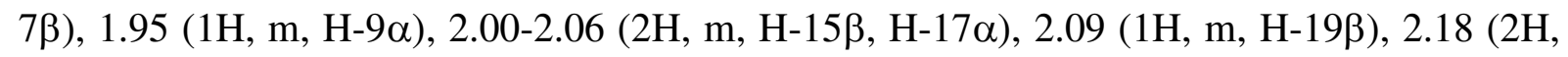
q, $\left.J=7.5 \mathrm{~Hz}, \mathrm{H}-2^{\prime}\right), 2.25$ (6H, bs, NB- $\left.\mathrm{CH}_{3}\right), 2.35(1 \mathrm{H}, \mathrm{d}, J=16.4 \mathrm{~Hz}, \mathrm{H}-12 \beta), 2.35(1 \mathrm{H}, \mathrm{m}$, $\mathrm{H}-2 \alpha), 2.56(1 \mathrm{H}, \mathrm{bd}, J=16.4 \mathrm{~Hz}, \mathrm{H}-12 \alpha), 2.61(1 \mathrm{H}, \mathrm{m}, \mathrm{H}-20), 3.26(1 \mathrm{H}, \mathrm{dd}, J=6.0,12.0$ $\mathrm{Hz}, \mathrm{H}-3 \alpha), 3.35$ (1H, d, $J=16.4 \mathrm{~Hz}, \mathrm{H}-19 \alpha) ; 3.77$ (1H, d, $J=10.4 \mathrm{~Hz}, \mathrm{H}-29 \alpha), 4.07$ (1H, d, $J=10.4 \mathrm{~Hz}, \mathrm{H}-29 \beta), 4.09(1 \mathrm{H}, \mathrm{m}, \mathrm{H}-16 \beta), 5.60(1 \mathrm{H}, \mathrm{m}, \mathrm{H}-1) ;{ }^{13} \mathrm{C} \mathrm{NMR}\left(100 \mathrm{MHz}, \mathrm{CDCl}_{3}\right) \delta$ $9.68\left(\mathrm{CH}_{3}, \mathrm{C}-30\right), 9.95\left(\mathrm{CH}_{3}, \mathrm{C}-21\right), 12.02\left(\mathrm{CH}_{3}, \mathrm{bs}, \mathrm{C}-4^{\prime}\right), 17.68\left(\mathrm{CH}_{3}\right.$, bs, C-5'), 17.79 
$\left(\mathrm{CH}_{3}, \mathrm{C}-18\right), 18.81\left(\mathrm{CH}_{3}, \mathrm{C}-28\right), 24.60\left(\mathrm{CH}_{2}, \mathrm{C}-6\right), 27.21\left(\mathrm{CH}_{2}, \mathrm{C}-3\right.$ ') $), 30.76\left(\mathrm{CH}_{2}, \mathrm{C}-2\right)$, 33.16 (C, C-4), $33.49\left(\mathrm{CH}_{2}, \mathrm{C}-7\right), 37.28\left(\mathrm{CH}_{2}, \mathrm{C}-19\right), 41.64\left(\mathrm{CH}, \mathrm{C}-2\right.$ '), $42.44\left(\mathrm{CH}_{2}, \mathrm{C}-15\right)$, 46.15 (C, C-14), 46.98 (C, C-13), 49.53 (CH, C-5), 49.68 (CH, C-8), $49.99\left(\mathrm{CH}_{2}, \mathrm{C}-12\right)$, $50.26(\mathrm{CH}, \mathrm{C}-9), 55.45(\mathrm{CH}, \mathrm{C}-17), 55.60(\mathrm{CH}, \mathrm{C}-3), 62.00(\mathrm{CH}, \mathrm{C}-20), 74.71\left(\mathrm{CH}_{2}, \mathrm{C}-29\right)$, 78.28 (CH, C-16), 121.87 (CH, C-1), 137.15 (C, C-10), 161.90 (C, C-1'), 211.61 (C, C-11); ES-MS m/z $499.3[\mathrm{M}+\mathrm{H}]^{+}(100), 500.4$ (20), 559.4 (40), $250.2[\mathrm{M}+\mathrm{H}]^{+} / 2$ (20); HRES-MS m/z 499.3900 (calcd for $\mathrm{C}_{31} \mathrm{H}_{51} \mathrm{~N}_{2} \mathrm{O}_{3}, 499.3900$ ).

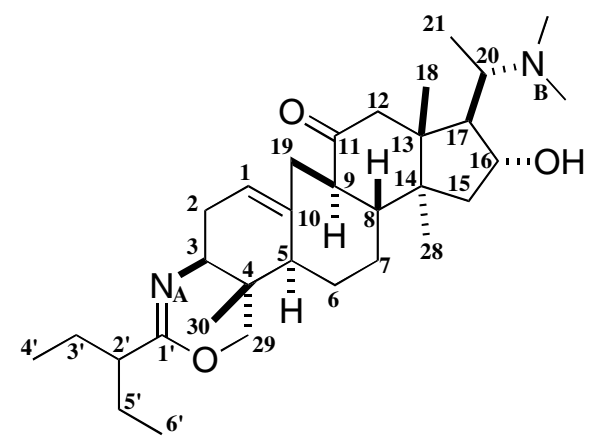

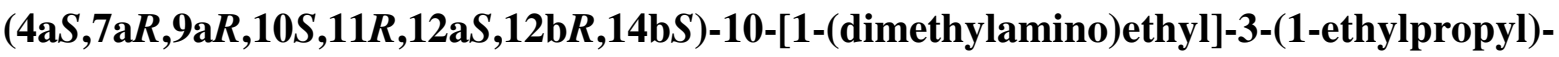
11-hydroxy-9a,12a,14b-trimethyl-1,4a,5,7,7a,9,9a,10,11,12,12a,12b,13,14,14a,14bhexadecahydro-8H-indeno[ $\left.5^{\prime \prime}, 4^{\prime \prime}: 4^{\prime}, 5^{\prime}\right]$ cyclohepta[1',2':3,4]benzo[1,2-d][1,3]o xazin-8-one (9f)

Compound 7f ( $85 \mathrm{mg}, 0.14 \mathrm{mmol}, 1$ equiv) was heated at $250{ }^{\circ} \mathrm{C}$ under $0.08 \mathrm{mmHg}$ in a rotating distillation oven with four bulbs. A product sublimated in 3 hours. The sublimate was composed of a mixture of the compound $\mathbf{8 f}(90 \%)$ and the expected dihydro-oxazine $9 \mathrm{f}$ $(10 \%)$. To a solution of the residue from the cap and the sublimate in $2 \mathrm{~mL}$ of dichloromethane was added $25 \%$ tetraethylammonium hydroxide $(472 \mathrm{mg}, 0.8 \mathrm{mmol}, 5$ equiv) solution in methanol. The solvent was evaporated under reduced pressure. The dark red residue was heated at $260{ }^{\circ} \mathrm{C}$ under $0.1 \mathrm{mmHg}$ in a rotating distillation oven with four bulbs. A product sublimated in 3 hours in three times to afford a sublimated pale yellow powder of 9f $(40 \mathrm{mg}, 49 \%)$ : IR $\left(\mathrm{CHCl}_{3}\right) \mathrm{U}_{\max }\left(\mathrm{cm}^{-1}\right): 3287,2938,1694,1658,1462,1346,1167 ;{ }^{1} \mathrm{H} \mathrm{NMR}$ $\left(400 \mathrm{MHz}, \mathrm{CDCl}_{3}\right) \delta 0.68(3 \mathrm{H}, \mathrm{s}, \mathrm{H}-18), 0.78(3 \mathrm{H}, \mathrm{s}, \mathrm{H}-30), 0.85(3 \mathrm{H}, \mathrm{d}, J=6.6 \mathrm{~Hz}, \mathrm{H}-21)$,

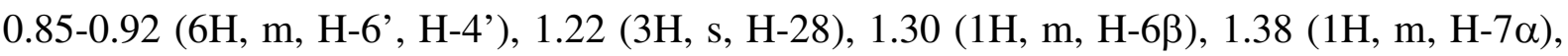

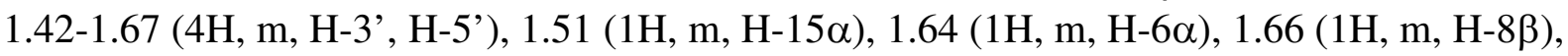

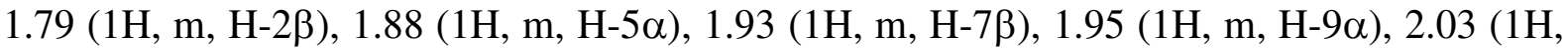

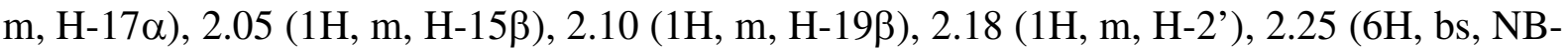
$\left.\mathrm{CH}_{3}\right), 2.33(1 \mathrm{H}, \mathrm{d}, J=16.3 \mathrm{~Hz}, \mathrm{H}-12 \beta), 2.35(1 \mathrm{H}, \mathrm{m}, \mathrm{H}-2 \alpha), 2.55(1 \mathrm{H}, \mathrm{bd}, J=16.3 \mathrm{~Hz}, \mathrm{H}-$ $12 \alpha), 2.59$ (1H, m, H-20), $3.26(1 \mathrm{H}, \mathrm{dd}, J=6.0,11.5 \mathrm{~Hz}, \mathrm{H}-3 \alpha), 3.33(1 \mathrm{H}, \mathrm{d}, J=15.5 \mathrm{~Hz}, \mathrm{H}-$

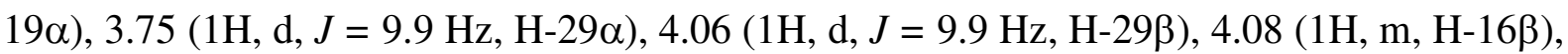
$5.59(1 \mathrm{H}, \mathrm{m}, \mathrm{H}-1) ;{ }^{13} \mathrm{C}$ NMR $\left(100 \mathrm{MHz}, \mathrm{CDCl}_{3}\right) \delta 9.98\left(\mathrm{CH}_{3}, \mathrm{C}-30\right), 12.09\left(\mathrm{CH}_{3}, \mathrm{C}-21\right)$, $12.15\left(\mathrm{CH}_{3}, \mathrm{C}-4\right.$ ', C-6'), $17.78\left(\mathrm{CH}_{3}, \mathrm{C}-18\right), 18.77\left(\mathrm{CH}_{3}, \mathrm{C}-28\right), 24.56\left(\mathrm{CH}_{2}, \mathrm{C}-6\right), 25.55$ $\left(\mathrm{CH}_{2}, \mathrm{C}-3\right.$ ', C-5'), $30.74\left(\mathrm{CH}_{2}, \mathrm{C}-2\right), 33.16(\mathrm{C}, \mathrm{C}-4), 33.48\left(\mathrm{CH}_{2}, \mathrm{C}-7\right), 37.28\left(\mathrm{CH}_{2}, \mathrm{C}-19\right)$, $42.47\left(\mathrm{CH}_{2}, \mathrm{C}-15\right), 46.18$ (C, C-14), 47.01 (C, C-13), 49.22 (CH, C-2'), 49.54 (CH, C-5), 49.67 (CH, C-8), $50.01\left(\mathrm{CH}_{2}, \mathrm{C}-12\right), 50.27$ (CH, C-9), 55.47 (CH, C-17), 55.82 (CH, C-3), 62.05 (CH, C-20), $74.59\left(\mathrm{CH}_{2}, \mathrm{C}-29\right), 78.24$ (CH, C-16), 121.87 (CH, C-1), 137.15 (C, C-10), 
161.90 (C, C-1'), 211.61 (C, C-11); ES-MS m/z 513.3 [M+H] ${ }^{+}$(100), 514.3 (50), 531.4 (10), $257.2[\mathrm{M}+\mathrm{H}]^{+} / 2$ (20), 257.7 (10); HRES-MS m/z 513.4045 (calcd for $\mathrm{C}_{32} \mathrm{H}_{53} \mathrm{~N}_{2} \mathrm{O}_{3}, 513.4056$ ).

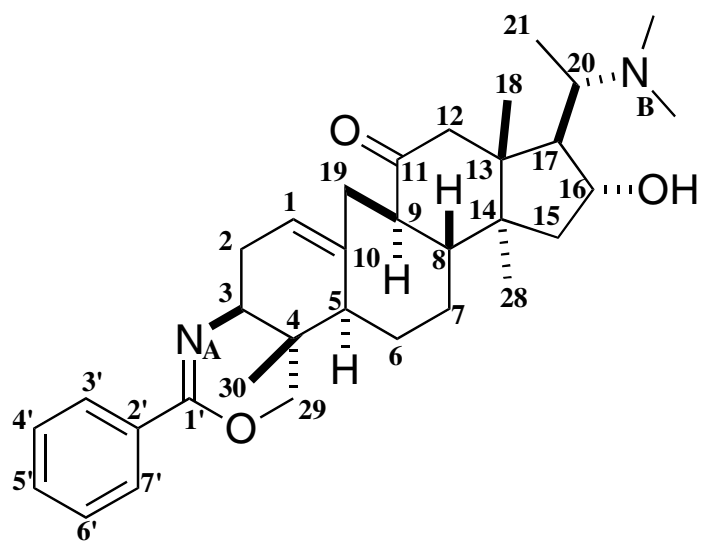

$(4 \mathrm{a} S, 7 \mathrm{a} R, 9 \mathrm{a} R, 10 S, 11 R, 12 \mathrm{a} S, 12 \mathrm{~b} R, 14 \mathrm{~b} S)-10-[1-(d i m e t h y l a m i n o) e t h y l]-3-p h e n y l-11-$ hydroxy-9a,12a,14b-trimethyl-1,4a,5,7,7a,9,9a,10,11,12,12a,12b,13,14,14a,14bhexadecahydro-8H-indeno[ $\left.5^{\prime \prime}, 4^{\prime \prime}: 4^{\prime}, 5^{\prime}\right]$ cyclohepta[1',2':3,4]benzo[1,2-d][1,3] oxazin-8-one $(9 g)$

To a solution of compound $7 \mathrm{~g}$ (118 $\mathrm{mg}, 0.22 \mathrm{mmol}, 1$ equiv) in $2 \mathrm{~mL}$ of dichloromethane was added $25 \%$ tetraethylammonium hydroxide $(648 \mathrm{mg}, 1.1 \mathrm{mmol}, 5$ equiv) solution in methanol. The solvent was evaporated under reduced pressure. The dark red residue was heated at $275{ }^{\circ} \mathrm{C}$ under $0.03 \mathrm{mmHg}$ in a rotating distillation oven with four bulbs. No product sublimated in 3 hours. This crude product from the cap was cristallized in acetone to afford a pale yellow powder of $9 \mathrm{~g}(97 \mathrm{mg}, 85 \%)$ : mp $249{ }^{\circ} \mathrm{C}$; $[\alpha]^{23}{ }_{\mathrm{D}}=+59\left(\mathrm{c} 0.3, \mathrm{CHCl}_{3}\right)$; IR $\left(\mathrm{CHCl}_{3}\right) \mathrm{u}_{\max }\left(\mathrm{cm}^{-1}\right): 3376,2977,1694,1650,1462,1375,1027 ;{ }^{1} \mathrm{H} \mathrm{NMR}\left(300 \mathrm{MHz}, \mathrm{CDCl}_{3}\right)$ $\delta 0.71(3 \mathrm{H}, \mathrm{s}, \mathrm{H}-18), 0.84(3 \mathrm{H}, \mathrm{s}, \mathrm{H}-30), 0.88(3 \mathrm{H}, \mathrm{d}, J=6.6 \mathrm{~Hz}, \mathrm{H}-21), 1.26(3 \mathrm{H}, \mathrm{s}, \mathrm{H}-28)$, $1.34(1 \mathrm{H}, \mathrm{m}, \mathrm{H}-6 \beta), 1.38(1 \mathrm{H}, \mathrm{m}, \mathrm{H}-7 \alpha), 1.53(1 \mathrm{H}, \mathrm{dd}, J=2.5,13.8 \mathrm{~Hz}, \mathrm{H}-15 \alpha), 1.67(1 \mathrm{H}$,

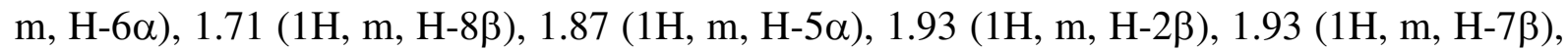

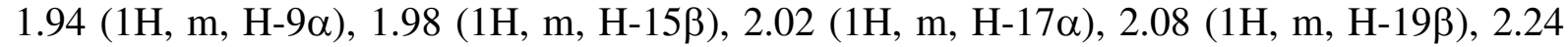
$\left(6 \mathrm{H}, \mathrm{bs}, \mathrm{NB}-\mathrm{CH}_{3}\right), 2.36(1 \mathrm{H}, \mathrm{d}, J=16.6 \mathrm{~Hz}, \mathrm{H}-12 \beta), 2.46(1 \mathrm{H}, \mathrm{m}, \mathrm{H}-2 \alpha), 2.58(1 \mathrm{H}, \mathrm{bd}, J=$ $16.6 \mathrm{~Hz}, \mathrm{H}-12 \alpha), 2.61(1 \mathrm{H}, \mathrm{m}, \mathrm{H}-20), 3.48(1 \mathrm{H}, \mathrm{dd}, J=7.1,14.0 \mathrm{~Hz}, \mathrm{H}-3 \alpha), 3.38(1 \mathrm{H}, \mathrm{d}, J=$

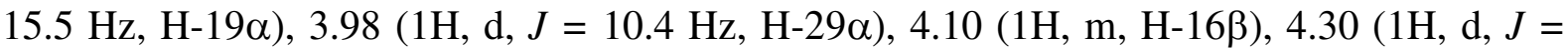
$10.4 \mathrm{~Hz}, \mathrm{H}-29 \beta), 5.67$ (1H, m, H-1), 7.37, 7.92 (5H, m, HAr = 3', 4', 5', 6', 7'); ${ }^{13} \mathrm{C}$ NMR $\left(75.5 \mathrm{MHz}, \mathrm{CDCl}_{3}\right) \delta 10.0\left(\mathrm{CH}_{3}, \mathrm{C}-30\right), 10.1\left(\mathrm{CH}_{3}, \mathrm{C}-21\right), 17.8\left(\mathrm{CH}_{3}, \mathrm{C}-18\right), 18.9\left(\mathrm{CH}_{3}, \mathrm{C}-\right.$ 28), $24.7\left(\mathrm{CH}_{2}, \mathrm{C}-6\right), 30.8\left(\mathrm{CH}_{2}, \mathrm{C}-2\right), 33.4(\mathrm{C}, \mathrm{C}-4), 33.5\left(\mathrm{CH}_{2}, \mathrm{C}-7\right), 37.3\left(\mathrm{CH}_{2}, \mathrm{C}-19\right), 42.5$ $\left(\mathrm{CH}_{2}, \mathrm{C}-15\right), 46.2(\mathrm{C}, \mathrm{C}-14), 47.0(\mathrm{C}, \mathrm{C}-13), 49.4(\mathrm{CH}, \mathrm{C}-5), 49.7(\mathrm{CH}, \mathrm{C}-8), 50.0\left(\mathrm{CH}_{2}, \mathrm{C}-\right.$ 12), 50.3 (CH, C-9), 55.5 (CH, C-17), $56.6(\mathrm{CH}, \mathrm{C}-3), 62.0(\mathrm{CH}, \mathrm{C}-20), 75.2\left(\mathrm{CH}_{2}, \mathrm{C}-29\right)$, 78.3 (CH, C-16), 121.9 (CH, C-1), 127.1, 128.0 (CH, C-Ar = 3', 4',6',7'), 130.3 (CH, C-5'), 133.5 (C, C-2'), 137.2 (C, C-10), 154.1 (C, C-1'), 211.7 (C, C-11); ES-MS m/z 519.4 [M+H] ${ }^{+}$ (100), 520.4 (10); HRES-MS $m / z, 519.3610$ (calcd for $\mathrm{C}_{33} \mathrm{H}_{47} \mathrm{~N}_{2} \mathrm{O}_{3}, 519.3587$ ); anal. C $73.25 \%, \mathrm{H} 9.56 \%$, N 5.98\%, calcd for $\mathrm{C}_{33} \mathrm{H}_{46} \mathrm{~N}_{2} \mathrm{O}_{3} \cdot \mathrm{H}_{2} \mathrm{O}$ C 73.84\%, H 9.01\%, N 5.22\%. 


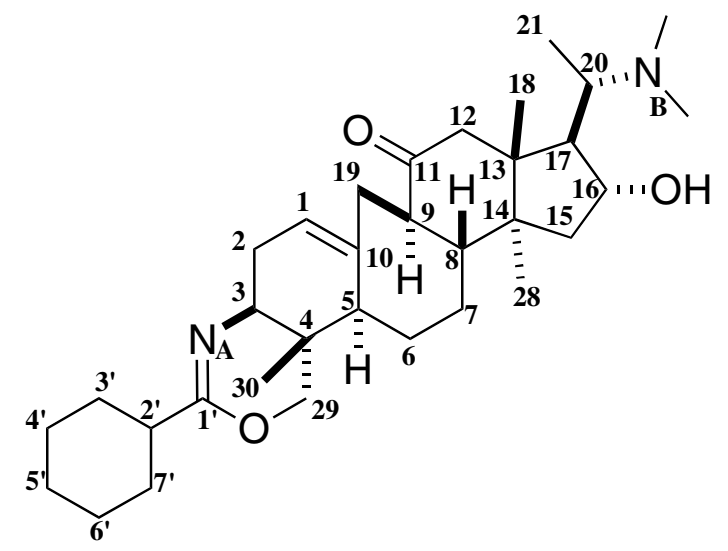

(4aS,7a $R, 9 \mathrm{a} R, 10 S, 11 R, 12 \mathrm{a} S, 12 \mathrm{~b} R, 14 \mathrm{~b} S)-10-[1-($ dimethylamino $)$ ethyl]-3-cyclohexyl-11hydroxy-9a,12a,14b-trimethyl-1,4a,5,7,7a,9,9a,10,11,12,12a,12b,13,14,14a,14b-hexadec ahydro-8H-indeno[ $\left.5^{\prime \prime}, 4^{\prime \prime}: 4^{\prime}, 5^{\prime}\right]$ cyclohepta[1',2' $\left.: 3,4\right]$ benzo[1,2-d][1,3]oxazin-8-one $(9 h)$

Compound $7 \mathrm{~h}$ ( $225 \mathrm{mg}, 0.41 \mathrm{mmol}, 1$ equiv) was heated at $260{ }^{\circ} \mathrm{C}$ under $0.03 \mathrm{mmHg}$ in a rotating distillation oven with four bulbs. No product sublimated in 3 hours. The sublimate was composed of a mixture of the compound $\mathbf{8 h}(83 \%)$ and the expected dihydro-oxazine $\mathbf{9 h}$ (17\%). To a solution of the residue from the cap and the sublimate in $2 \mathrm{~mL}$ of dichloromethane was $25 \%$ tetraethylammonium hydroxide (1085 mg, $1.8 \mathrm{mmol}, 5$ equiv) solution in methanol. The solvent was evaporated under reduced pressure. The dark red residue was heated at $260{ }^{\circ} \mathrm{C}$ under $0.03 \mathrm{mmHg}$ in a rotating distillation oven with four bulbs. A product sublimated in 3 hours to afford a pale yellow powder of $\mathbf{9 h}(150 \mathrm{mg}, 69 \%)$ : $\mathrm{mp} 270$ ${ }^{\circ} \mathrm{C} ;[\alpha]^{23}{ }_{\mathrm{D}}=+72\left(\mathrm{c} 0.1, \mathrm{CHCl}_{3}\right) ; \mathrm{IR}\left(\mathrm{CHCl}_{3}\right) \mathrm{v}_{\max }\left(\mathrm{cm}^{-1}\right): 3433,2936,1693,1659,1451,1389$, 1040; ${ }^{1} \mathrm{H}$ NMR $\left(300 \mathrm{MHz}, \mathrm{CDCl}_{3}\right) \delta 0.70(3 \mathrm{H}, \mathrm{s}, \mathrm{H}-18), 0.75(3 \mathrm{H}, \mathrm{s}, \mathrm{H}-30), 0.87(3 \mathrm{H}, \mathrm{d}, J=$ $6.6 \mathrm{~Hz}, \mathrm{H}-21), 1.24$ (3H, s, H-28), $1.27(1 \mathrm{H}, \mathrm{m}, \mathrm{H}-6 \beta), 1.35(1 \mathrm{H}, \mathrm{m}, \mathrm{H}-7 \alpha), 1.51(1 \mathrm{H}, \mathrm{dd}, J=$

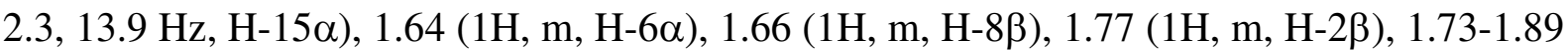

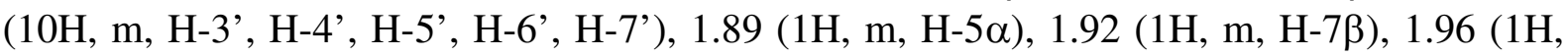

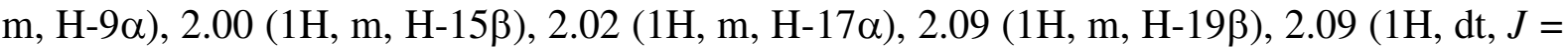
4.0, $12.3 \mathrm{~Hz}, \mathrm{H}-2$ ') 2.24 (6H, bs, NB-CH3), 2.34 (1H, d, $J=15.8 \mathrm{~Hz}, \mathrm{H}-12 \beta), 2.36$ (1H, m, H-2 $\alpha), 2.56(1 \mathrm{H}, \mathrm{bd}, J=15.8 \mathrm{~Hz}, \mathrm{H}-12 \alpha), 2.60(1 \mathrm{H}, \mathrm{dd}, J=6.6,10.7 \mathrm{~Hz}, \mathrm{H}-20), 3.24(1 \mathrm{H}$, $\mathrm{dd}, J=5.8,11.6 \mathrm{~Hz}, \mathrm{H}-3 \alpha), 3.34(1 \mathrm{H}, \mathrm{d}, J=15.4 \mathrm{~Hz}, \mathrm{H}-19 \alpha), 3.76(1 \mathrm{H}, \mathrm{d}, J=10.2 \mathrm{~Hz}, \mathrm{H}-$ $29 \alpha), 4.06$ (1H, d, $J=10.2 \mathrm{~Hz}, \mathrm{H}-29 \beta), 4.09$ (1H, m, H-16ß), 5.60 (1H, m, H-1); ${ }^{13} \mathrm{C} \mathrm{NMR}$ (75.5 MHz, $\left.\mathrm{CDCl}_{3}\right) \delta 9.6\left(\mathrm{CH}_{3}, \mathrm{C}-30\right), 10.0\left(\mathrm{CH}_{3}, \mathrm{C}-21\right), 17.8\left(\mathrm{CH}_{3}, \mathrm{C}-18\right), 18.8\left(\mathrm{CH}_{3}, \mathrm{C}-28\right)$, $24.6\left(\mathrm{CH}_{2}, \mathrm{C}-6\right), 25.8,25.9\left(\mathrm{CH}_{2}, \mathrm{C}-4\right.$ ', C-6'), 29.9, $30.0\left(\mathrm{CH}_{2}, \mathrm{C}-5^{\prime}, \mathrm{C}-3\right.$ ', C-7'), $30.8\left(\mathrm{CH}_{2}\right.$, C-2), 32.9 (C, C-4), $33.5\left(\mathrm{CH}_{2}, \mathrm{C}-7\right), 37.3\left(\mathrm{CH}_{2}, \mathrm{C}-19\right), 42.4\left(\mathrm{CH}_{2}, \mathrm{C}-15\right), 44.1$ (CH, C-2'), 46.1 (C, C-14), 47.0 (C, C-13), 49.4 (CH, C-5), 49.7 (CH, C-8), $50.0\left(\mathrm{CH}_{2}, \mathrm{C}-12\right), 50.2(\mathrm{CH}$, C-9), $55.4(\mathrm{CH}, \mathrm{C}-17), 55.6(\mathrm{CH}, \mathrm{C}-3), 62.0(\mathrm{CH}, \mathrm{C}-20), 74.7\left(\mathrm{CH}_{2}, \mathrm{C}-29\right), 78.3(\mathrm{CH}, \mathrm{C}-16)$, 121.8 (CH, C-1), 137.2 (C, C-10), 156.4 (C, C-1'), 211.6 (C, C-11); ES-MS m/z 525.3 $[\mathrm{M}+\mathrm{H}]^{+}$(100), 526.4 (40), 543.4 (50); HRES-MS m/z 525.4041 (calcd for $\mathrm{C}_{33} \mathrm{H}_{53} \mathrm{~N}_{2} \mathrm{O}_{3}$, 525.4056). 


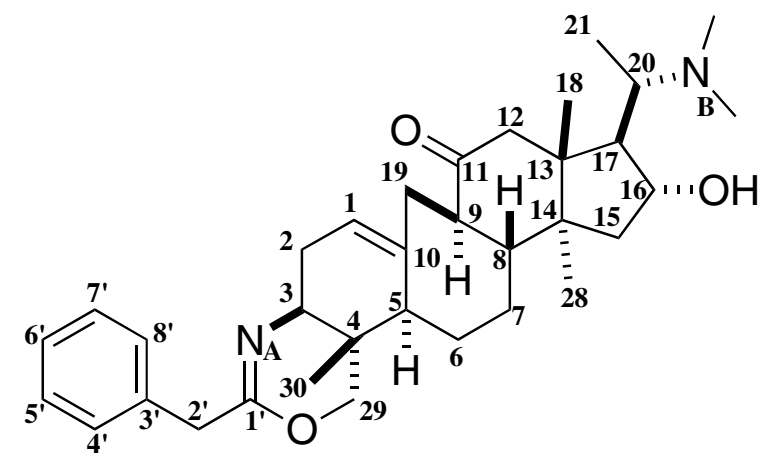

$(4 \mathrm{a} S, 7 \mathrm{a} R, 9 \mathrm{a} R, 10 S, 11 R, 12 \mathrm{a} S, 12 \mathrm{~b} R, 14 \mathrm{~b} S)-10-[1-(d i m e t h y l a m i n o) e t h y l]-3-b e n z y l-11-$ hydroxy-9a,12a,14b-trimethyl-1,4a,5,7,7a,9,9a,10,11,12,12a,12b,13,14,14a,14b-hexadec ahydro-8H-indeno[5", $\left.4^{\prime \prime}: 4^{\prime}, 5^{\prime}\right]$ cyclohepta[1',2':3,4]benzo[1,2-d][1,3]oxazin-8-one (9i)

Compound 7i (240 mg, $0.43 \mathrm{mmol}, 1$ equiv) was heated at $260{ }^{\circ} \mathrm{C}$ under $0.03 \mathrm{mmHg}$ in a rotating distillation oven with four bulbs. No product sublimated in 3 hours. The residue from the cap was composed of a mixture of the compound 8i (74\%) and the expected dihydrooxazine $9 \mathbf{i}(26 \%)$. To a solution of the residue in $2 \mathrm{~mL}$ of dichloromethane was added of $25 \%$ tetraethylammonium hydroxide $(926 \mathrm{mg}, 1.57 \mathrm{mmol}, 5$ equiv) solution in methanol. The solvent was evaporated under reduced pressure. The dark red residue was heated at $260{ }^{\circ} \mathrm{C}$ under $0.03 \mathrm{mmHg}$ in a rotating distillation oven with four bulbs. A product sublimated in 3 hours to afford a sublimated pale yellow powder of $9 \mathbf{i}(125 \mathrm{mg}, 55 \%)$ : IR $\left(\mathrm{CHCl}_{3}\right) \cup_{\max }\left(\mathrm{cm}^{-1}\right)$ : 3411, 2940, 1694, 1665, 1454, 1375, 1039; $\left.{ }^{1} \mathrm{H} \mathrm{NMR} \mathrm{(400} \mathrm{MHz,} \mathrm{CDCl}_{3}\right) \delta 0.69$ (3H, s, H-18),

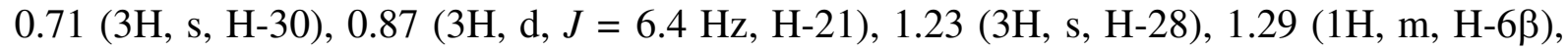
$1.33(1 \mathrm{H}, \mathrm{m}, \mathrm{H}-7 \alpha), 1.49(1 \mathrm{H}, \mathrm{dd}, J=2.6,13.9 \mathrm{~Hz}, \mathrm{H}-15 \alpha), 1.61(1 \mathrm{H}, \mathrm{m}, \mathrm{H}-6 \alpha), 1.65(1 \mathrm{H}$,

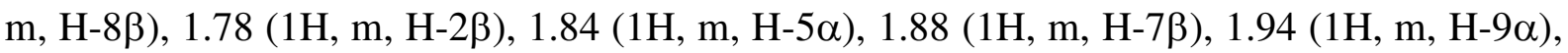
$2.00(1 \mathrm{H}, \mathrm{m}, \mathrm{H}-15 \beta), 2.03(1 \mathrm{H}, \mathrm{m}, \mathrm{H}-17 \alpha), 2.10(1 \mathrm{H}, \mathrm{m}, \mathrm{H}-19 \beta), 2.24\left(6 \mathrm{H}, \mathrm{bs}, \mathrm{NB}-\mathrm{CH}_{3}\right)$, $2.34(1 \mathrm{H}, \mathrm{d}, J=15.8 \mathrm{~Hz}, \mathrm{H}-12 \beta), 2.41(1 \mathrm{H}, \mathrm{m}, \mathrm{H}-2 \alpha), 2.55(1 \mathrm{H}, \mathrm{bd}, J=15.8 \mathrm{~Hz}, \mathrm{H}-12 \alpha)$, $2.58(1 \mathrm{H}, \mathrm{dd}, J=6.4,10.6 \mathrm{~Hz}, \mathrm{H}-20), 3.27(1 \mathrm{H}, \mathrm{dd}, J=6.2,12.0 \mathrm{~Hz}, \mathrm{H}-3 \alpha), 3.34(1 \mathrm{H}, \mathrm{d}, J=$ $15.4 \mathrm{~Hz}, \mathrm{H}-19 \alpha), 3.47,3.49$ (2H, 2d, $J=14.9 \mathrm{~Hz}, \mathrm{H}-2$ '), 3.77 (1H, d, $J=10.0 \mathrm{~Hz}, \mathrm{H}-29 \alpha)$, $4.04(1 \mathrm{H}, \mathrm{d}, J=10.0 \mathrm{~Hz}, \mathrm{H}-29 \beta), 4.08(1 \mathrm{H}, \mathrm{m}, \mathrm{H}-16 \beta), 5.60(1 \mathrm{H}, \mathrm{m}, \mathrm{H}-1), 7.25-7.32$ (5H, m, H-4', H-5', H-6', H-7', H-8'); ${ }^{13} \mathrm{C}$ NMR (100 MHz, $\left.\mathrm{CDCl}_{3}\right) \delta 9.8\left(\mathrm{CH}_{3}, \mathrm{C}-30\right), 10.0\left(\mathrm{CH}_{3}, \mathrm{C}-\right.$ 21), $17.8\left(\mathrm{CH}_{3}, \mathrm{C}-18\right), 18.8\left(\mathrm{CH}_{3}, \mathrm{C}-28\right), 24.6\left(\mathrm{CH}_{2}, \mathrm{C}-6\right), 30.6\left(\mathrm{CH}_{2}, \mathrm{C}-2\right), 33.1(\mathrm{C}, \mathrm{C}-4)$, $33.5\left(\mathrm{CH}_{2}, \mathrm{C}-7\right), 37.3\left(\mathrm{CH}_{2}, \mathrm{C}-19\right), 42.0\left(\mathrm{CH}_{2}, \mathrm{C}-2{ }^{\prime}\right), 42.4\left(\mathrm{CH}_{2}, \mathrm{C}-15\right), 46.1(\mathrm{C}, \mathrm{C}-14), 47.0$ $(\mathrm{C}, \mathrm{C}-13), 49.3(\mathrm{CH}, \mathrm{C}-5), 49.7(\mathrm{CH}, \mathrm{C}-8), 50.0\left(\mathrm{CH}_{2}, \mathrm{C}-12\right), 50.2(\mathrm{CH}, \mathrm{C}-9), 55.4(\mathrm{CH}, \mathrm{C}-$ 17), $56.0(\mathrm{CH}, \mathrm{C}-3), 62.0(\mathrm{CH}, \mathrm{C}-20), 75.1\left(\mathrm{CH}_{2}, \mathrm{C}-29\right), 78.3(\mathrm{CH}, \mathrm{C}-16), 121.7(\mathrm{CH}, \mathrm{C}-1)$, 126.6 (CH, C-6'), 128.4 (CH, C-4', C-8'), 128.8 (CH, C-5', C-7'), 129.3 (C, C-3'), 137.2 (C, C-10), 157.7 (C, C-1'), 211.7 (C, C-11); ES-MS m/z 533.3 [M+H] ${ }^{+}$(100), 534.4 (50); HRESMS $m / z 533.3747$ (calcd for $\mathrm{C}_{34} \mathrm{H}_{49} \mathrm{~N}_{2} \mathrm{O}_{3}, 533.3743$ ). 


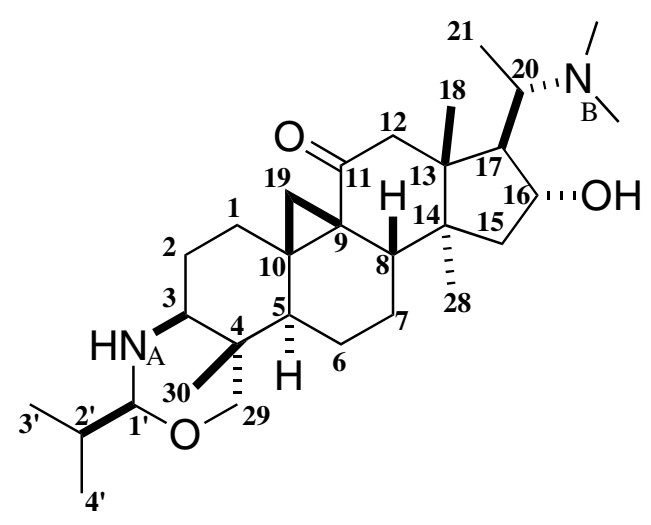

$(3 S, 4 \mathrm{a} S, 6 \mathrm{a} R, 7 \mathrm{a} R, 9 \mathrm{a} R, 10 S, 11 R, 12 \mathrm{a} S, 12 \mathrm{~b} S, 14 \mathrm{a} R, 14 \mathrm{~b} S)-10-[1-(d i m e t h y l a m i n o) e t h y l]-11-$ hydroxy-3-isopropyl-9a,12a,14b-trimethylhexadecahydro- $1 \mathrm{H}, 8 \mathrm{H}$-cyclopenta[7,8]cyclo propa $[4 a, 4 b]$ phenanthro[2,1-d][1,3]oxazin-8-one (10)

To a suspension of cycloxobuxidine-F, compound 5 ( $87 \mathrm{mg}, 0.2 \mathrm{mmol}, 1$ equiv) in $15 \mathrm{~mL}$ of freshly distilled 1,4-dioxane was added isobutyraldehyde ( $37 \mu \mathrm{L}, 0.40 \mathrm{mmol}, 2$ equiv). After stirring at $50{ }^{\circ} \mathrm{C}$ for 12 hours, then cooling to room temperature, the solvent was evaporated under reduced pressure. The residue dissolved in $20 \mathrm{~mL}$ of dichloromethane was then made basic with $40 \mathrm{~mL}$ of a $10 \%$ sodium bicarbonate solution and extracted with dichloromethane $(3 \times 40 \mathrm{~mL})$. The organic layer was dried over $\mathrm{Na}_{2} \mathrm{SO}_{4}$ and filtered, and the solvent was evaporated to give a residue $(105 \mathrm{mg})$. A trituration of the crude product in diethyl ether afforded a colorless powder of $\mathbf{1 0}(28 \mathrm{mg}, 30 \%)$ : $\mathrm{mp} 229^{\circ} \mathrm{C}$; $[\alpha]^{23}{ }_{\mathrm{D}}=+94\left(\mathrm{c} 1.4, \mathrm{CHCl}_{3}\right)$; IR $\left(\mathrm{CHCl}_{3}\right) \cup_{\max }\left(\mathrm{cm}^{-1}\right): 3319,2950,1663,1466,1368,1096 ;{ }^{1} \mathrm{H}$ NMR $\left(300 \mathrm{MHz}, \mathrm{CDCl}_{3}\right) \delta 0.83$ $(3 \mathrm{H}, \mathrm{s}, \mathrm{H}-18), 0.88$ (3H, d, $J=6.4 \mathrm{~Hz}, \mathrm{H}-21), 0.93(3 \mathrm{H}, \mathrm{s}, \mathrm{H}-30), 0.97(6 \mathrm{H}, \mathrm{d}, J=6.8 \mathrm{~Hz}, \mathrm{H}-$ 3', H-4'), 1.05 (1H, m, H-6ß), 1.07 (1H, d, $J=3.6 \mathrm{~Hz}, \mathrm{H}-19 \alpha), 1.22$ (3H, s, H-28), 1.28 (1H, m, H-1 $\alpha), 1.30(1 \mathrm{H}, \mathrm{m}, \mathrm{H}-7 \alpha), 1.40(5 \mathrm{H}, \mathrm{m}, \mathrm{H}-7 \beta), 1.45(1 \mathrm{H}, \mathrm{dd}, J=2.6,13.9 \mathrm{~Hz}, \mathrm{H}-15 \alpha)$, $1.53(1 \mathrm{H}, \mathrm{m}, \mathrm{H}-2 \beta), 1.58(1 \mathrm{H}, \mathrm{m}, \mathrm{H}-2 \alpha), 1.60(1 \mathrm{H}, \mathrm{m}, \mathrm{H}-6 \alpha), 1.61(1 \mathrm{H}, \mathrm{d}, J=3.6 \mathrm{~Hz}, \mathrm{H}-$

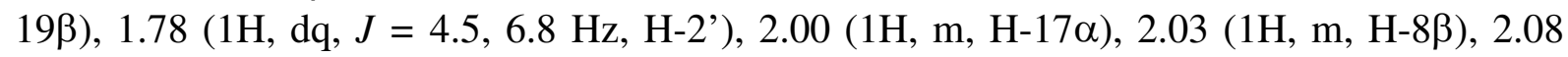
$(1 \mathrm{H}, \mathrm{m}, \mathrm{H}-15 \beta), 2.11(1 \mathrm{H}, \mathrm{m}, \mathrm{H}-5 \alpha), 2.28\left(6 \mathrm{H}, \mathrm{bs}, \mathrm{NB}-\mathrm{CH}_{3}\right), 2.33(1 \mathrm{H}, \mathrm{d}, J=17.0 \mathrm{~Hz}, \mathrm{H}-$ $12 \beta), 2.33(1 \mathrm{H}, \mathrm{m}, \mathrm{H}-1 \beta), 2.50(1 \mathrm{H}, \mathrm{bd}, J=17.0 \mathrm{~Hz}, \mathrm{H}-12 \alpha), 2.60(1 \mathrm{H}, \mathrm{m}, J=3.2,11.5 \mathrm{~Hz}$, $\mathrm{H}-3), 2.61(1 \mathrm{H}, \mathrm{m}, J=6.4 \mathrm{~Hz}, \mathrm{H}-20), 3.30(1 \mathrm{H}, \mathrm{d}, J=10.7 \mathrm{~Hz}, \mathrm{H}-29 \alpha), 3.82(1 \mathrm{H}, \mathrm{d}, J=10.7$ $\mathrm{Hz}, \mathrm{H}-29 \beta), 3.91\left(1 \mathrm{H}, \mathrm{d}, J=4.5 \mathrm{~Hz}, \mathrm{H}-1 \alpha^{\prime}\right), 4.11(1 \mathrm{H}, \mathrm{m}, J=7.0 \mathrm{~Hz}, \mathrm{H}-16 \beta) ;{ }^{13} \mathrm{C} \mathrm{NMR}$ $\left(300 \mathrm{MHz}, \mathrm{CDCl}_{3}\right) \delta 10.0\left(\mathrm{CH}_{3}, \mathrm{C}-21\right), 11.3\left(\mathrm{CH}_{3}, \mathrm{C}-30\right), 17.4\left(\mathrm{CH}_{3}, \mathrm{C}-18\right), 17.7,17.8\left(\mathrm{CH}_{3}\right.$, C-3', C-4'), $17.9\left(\mathrm{CH}_{2}, \mathrm{C}-6\right), 20.6\left(\mathrm{CH}_{3}, \mathrm{C}-28\right), 23.9\left(\mathrm{CH}_{2}, \mathrm{C}-7\right), 27.5\left(\mathrm{CH}_{2}, \mathrm{C}-2\right), 28.3\left(\mathrm{CH}_{2}\right.$, C-1), $30.2\left(\mathrm{CH}_{2}, \mathrm{C}-19\right), 32.7$ (CH, C-2'), 34.3 (C, C-9), 37.3 (C, C-4), 37.6 (C, C-10), 40.8 $(\mathrm{CH}, \mathrm{C}-8), 42.7\left(\mathrm{CH}_{2}, \mathrm{C}-15\right), 44.6(\mathrm{C}, \mathrm{C}-13), 45.3(\mathrm{CH}, \mathrm{C}-5), 47.2(\mathrm{C}, \mathrm{C}-14), 51.4\left(\mathrm{CH}_{2}, \mathrm{C}-\right.$ 12), 55.8 (CH, C-17), 62.1 (CH, C-20), $62.7(\mathrm{CH}, \mathrm{C}-3), 77.2\left(\mathrm{CH}_{2}, \mathrm{C}-29\right), 78.2(\mathrm{CH}, \mathrm{C}-16)$, 92.6 (CH, C-1'), 210.8 (C, C-11); ES-MS m/z 487.4 [M+H] ${ }^{+}$(100), 488.4 (30); HRES-MS $m / z 487.3897$ (calcd for $\mathrm{C}_{30} \mathrm{H}_{51} \mathrm{~N}_{2} \mathrm{O}_{3}, 487.3900$ ). 


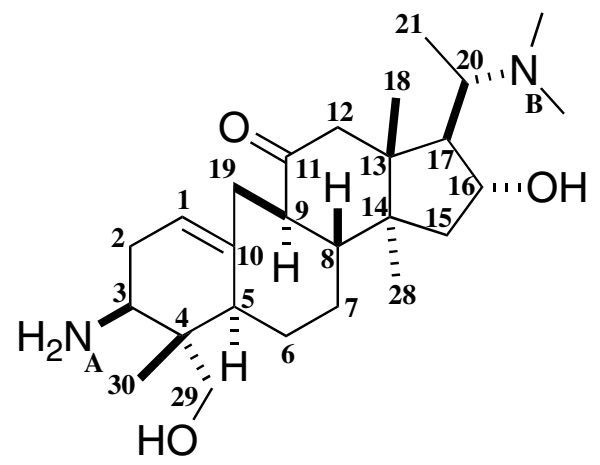

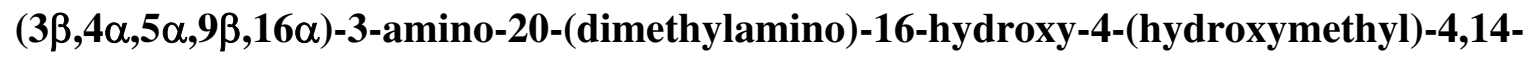 dimethyl-9,19-cyclopregnan-11-one (11)}

Compound 2 ( $250 \mathrm{mg}, 0.5 \mathrm{mmol}, 1$ equiv) was heated at $245{ }^{\circ} \mathrm{C}$ under $0.1 \mathrm{mmHg}$ in a rotating distillation oven with four bulbs. A product sublimated in 3 hours. The sublimate was composed of a mixture of the product $4(18 \%)$ and dihydro-oxazine $1(82 \%)$. To a suspension of the sublimate $(250 \mathrm{mg})$ in $15 \mathrm{~mL}$ of methanol was added $36 \mathrm{~mL}$ of $2 \mathrm{~N}$ sulfuric acid dropwise at room temperature. The stirred mixture was refluxed at $90{ }^{\circ} \mathrm{C}$ for 2 hours, then cooled at room temperature. The methanol was evaporated under reduced pressure at $25{ }^{\circ} \mathrm{C}$ at the most. The liquid solution was stirred at $80{ }^{\circ} \mathrm{C}$ for 1 hour. The mixture was then made basic with $40 \mathrm{~mL}$ of a $10 \%$ ammonia solution and extracted with dichloromethane $(3 \times 40 \mathrm{~mL})$. The organic layer was dried over $\mathrm{Na}_{2} \mathrm{SO}_{4}$ and filtered, and the solvent was evaporated to give a residue. A trituration of the crude product in ethyl acetate afforded a colorless powder of $\mathbf{1 1}$ (128 mg, 60\%) whose spectroscopic characteristics were consistent with those of HerlemGaulier, D.; Kuong-Huu-Lainé, F.; Goutarel, R.; Magdeleine, M.J. Alcaloïdes stéroïdiques LXXII. Alcaloïdes du groupe des cylcloxobuxidines retirés du Buxus balearica Willd : N-3isobutyryl cycloxobuxidine-F (baléabuxidine), $N$-3-isobutyryl cycloxobuxidine-H, N-3benzoyl cycloxobuxidine-F et cycloxobuxoxazine-C Bull. Soc. Chim. Fr. 1968, 2, 763-773. $\operatorname{mp} 266{ }^{\circ} \mathrm{C} ;[\alpha]^{23}{ }_{\mathrm{D}}=+29\left(\mathrm{c} 0.82, \mathrm{CHCl}_{3}\right) ; \mathrm{IR}\left(\mathrm{CHCl}_{3}\right) \mathrm{v}_{\max }\left(\mathrm{cm}^{-1}\right): 3383,2968,1693,1662$, $1602,1463,1374,1038 ;{ }^{1} \mathrm{H}$ NMR $\left(300 \mathrm{MHz}, \mathrm{CDCl}_{3}\right) \delta 0.69(3 \mathrm{H}, \mathrm{s}, \mathrm{H}-18), 0.78(3 \mathrm{H}, \mathrm{s}, \mathrm{H}-$ 30), $0.87(3 \mathrm{H}, \mathrm{d}, J=6.6 \mathrm{~Hz}, \mathrm{H}-21), 1.25(3 \mathrm{H}, \mathrm{s}, \mathrm{H}-28), 1.30(1 \mathrm{H}, \mathrm{m}, \mathrm{H}-6 \beta), 1.50(1 \mathrm{H}, \mathrm{m}, J=$

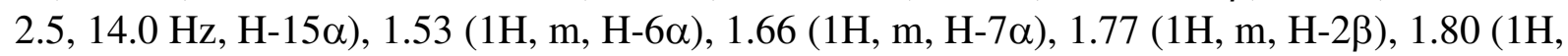

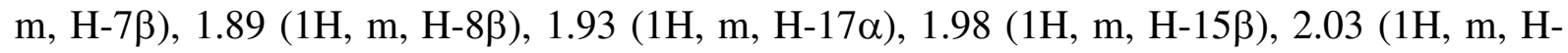
$9 \alpha), 2.07$ (1H, m, H-19ß), $2.12(1 \mathrm{H}, \mathrm{m}, \mathrm{H}-2 \alpha), 2.24\left(6 \mathrm{H}, \mathrm{bs}, \mathrm{NB}-\mathrm{CH}_{3}\right), 2.32(1 \mathrm{H}, \mathrm{m}, \mathrm{H}-5 \alpha)$, $2.34(1 \mathrm{H}, \mathrm{d}, J=15.6 \mathrm{~Hz}, \mathrm{H}-12 \beta), 2.56(1 \mathrm{H}, \mathrm{bd}, J=15.6 \mathrm{~Hz}, \mathrm{H}-12 \alpha), 2.59(1 \mathrm{H}, \mathrm{dq}, J=6.4$, $11.8 \mathrm{~Hz}, \mathrm{H}-20), 2.84$ (1H, dd, $J=5.8,10.5 \mathrm{~Hz}, \mathrm{H}-3 \alpha), 3.30$ (1H, d, $J=15.2 \mathrm{~Hz}, \mathrm{H}-19 \alpha), 3.58$ $(1 \mathrm{H}, \mathrm{d}, J=11.0 \mathrm{~Hz}, \mathrm{H}-29 \alpha), 3.76(1 \mathrm{H}, \mathrm{d}, J=11.0 \mathrm{~Hz}, \mathrm{H}-29 \beta), 4.09(1 \mathrm{H}, \mathrm{dd}, J=2.4,6.0,9.0$ $\mathrm{Hz}, \mathrm{H}-16 \beta), 5.48(1 \mathrm{H}, \mathrm{m}, \mathrm{H}-1) ;{ }^{13} \mathrm{C} \mathrm{NMR}\left(300 \mathrm{MHz}, \mathrm{CDCl}_{3}\right) \delta 8.4\left(\mathrm{CH}_{3}, \mathrm{C}-30\right), 10.0\left(\mathrm{CH}_{3}\right.$, C-21), $17.8\left(\mathrm{CH}_{3}, \mathrm{C}-18\right), 20.8\left(\mathrm{CH}_{3}, \mathrm{C}-28\right), 25.3\left(\mathrm{CH}_{2}, \mathrm{C}-6\right), 30.5\left(\mathrm{CH}_{2}, \mathrm{C}-2\right), 33.5\left(\mathrm{CH}_{2}, \mathrm{C}-\right.$ 7), $37.3\left(\mathrm{CH}_{2}, \mathrm{C}-19\right), 41.5$ (C, C-4), $42.4\left(\mathrm{CH}_{2}, \mathrm{C}-15\right), 42.8(\mathrm{CH}, \mathrm{C}-5), 46.2(\mathrm{C}, \mathrm{C}-14), 47.0$ (C, C-13), $49.7(\mathrm{CH}, \mathrm{C}-8), 50.0\left(\mathrm{CH}_{2}, \mathrm{C}-12\right), 50.1(\mathrm{CH}, \mathrm{C}-9), 55.3(\mathrm{CH}, \mathrm{C}-3), 55.5(\mathrm{CH}, \mathrm{C}-$ 17), 62.0 (CH, C-20), $71.7\left(\mathrm{CH}_{2}, \mathrm{C}-29\right), 78.4(\mathrm{CH}, \mathrm{C}-16), 119.8$ (CH, C-1), 138.3 (C, C-10), 212.1 (C, C-11); ES-MS m/z $433.4[\mathrm{M}+\mathrm{H}]^{+}$(100); HRES-MS $\mathrm{m} / \mathrm{z} 433.3415$ (calcd for $\mathrm{C}_{26} \mathrm{H}_{45} \mathrm{~N}_{2} \mathrm{O}_{3}, 433.3430$ ). 


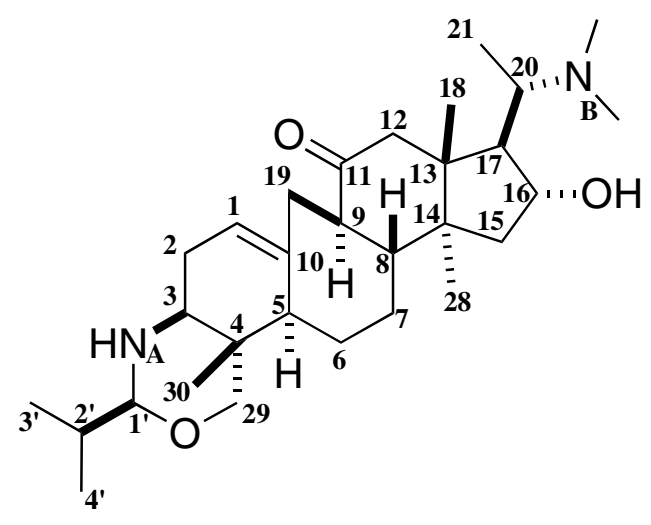

$(3 S, 4 \mathrm{a} S, 6 \mathrm{a} S, 7 \mathrm{a} R, 9 \mathrm{a} R, 10 S, 11 R, 12 \mathrm{a} S, 12 \mathrm{~b} R, 14 \mathrm{a} R, 14 \mathrm{~b} S)-10-[1-(d i m e t h y l a m i n o) e t h y l]-11-$ hydroxy-3-isopropyl-9a,12a,14b-trimethylicosahydro-8H-indeno[5",4": $4^{\prime}, 5$ '] cyclo hepta[1',2':3,4]benzo[1,2- $d][1,3]$ oxazin-8-one

(12)

To a suspension of compound 11 (85 $\mathrm{mg}, 0.2 \mathrm{mmol}, 1$ equiv) in $10 \mathrm{~mL}$ of freshly distilled 1,4-dioxane was added isobutyraldehyde $\left(37 \mu \mathrm{L}, 0.40 \mathrm{mmol}, 2\right.$ equiv). After stirring at $50{ }^{\circ} \mathrm{C}$ for 12 hours, then cooling to room temperature, the solvent was evaporated under reduced pressure. The residue dissolved in $20 \mathrm{~mL}$ of dichloromethane was then made basic with 40 $\mathrm{mL}$ of a $10 \%$ sodium bicarbonate solution and extracted with dichloromethane $(3 \times 40 \mathrm{~mL})$. The organic layer was dried over $\mathrm{Na}_{2} \mathrm{SO}_{4}$ and filtered, and the solvent was evaporated to give a residue $(94 \mathrm{mg}$ ). A trituration of the crude product in diethyl ether afforded a pale yellow powder of 12 (40 mg, 42\%) whose spectroscopic characteristics were consistent with those of Herlem-Gaulier, D.; Kuong-Huu-Lainé, F.; Goutarel, R.; Magdeleine, M.J. Alcaloïdes stéroïdiques LXXII. Alcaloïdes du groupe des cylcloxobuxidines retirés du Buxus balearica Willd : $N$-3-isobutyryl cycloxobuxidine-F (baléabuxidine), $N$-3-isobutyryl cycloxobuxidine-H, N-3-benzoyl cycloxobuxidine-F et cycloxobuxoxazine-C Bull. Soc. Chim. Fr. 1968, 2, $763-$ 773.: $\mathrm{mp} 253{ }^{\circ} \mathrm{C} ;[\alpha]^{23}{ }_{\mathrm{D}}=+65\left(\mathrm{c} 0.74, \mathrm{CHCl}_{3}\right) ; \mathrm{IR}\left(\mathrm{CHCl}_{3}\right) \mathrm{v}_{\max }\left(\mathrm{cm}^{-1}\right): 3442,2400,1693$, 1521, 1474, 1391, 1044; ${ }^{1} \mathrm{H}$ NMR $\left(300 \mathrm{MHz}, \mathrm{CDCl}_{3}\right) \delta 0.69(3 \mathrm{H}, \mathrm{s}, \mathrm{H}-18), 0.80(3 \mathrm{H}, \mathrm{s}, \mathrm{H}-$ 30), 0.87 (3H, d, $J=6.4 \mathrm{~Hz}, \mathrm{H}-21), 0.97$ (6H, d, $J=5.8 \mathrm{~Hz}, \mathrm{H}-3$ ', H-4'), 1.25 (3H, s, H-28), $1.29(1 \mathrm{H}, \mathrm{m}, \mathrm{H}-6 \beta), 1.36(1 \mathrm{H}, \mathrm{m}, \mathrm{H}-7 \alpha), 1.50(1 \mathrm{H}, \mathrm{dd}, J=2.6,13.9 \mathrm{~Hz}, \mathrm{H}-15 \alpha), 1.58(1 \mathrm{H}$,

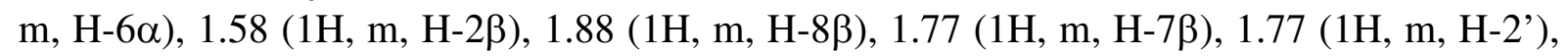

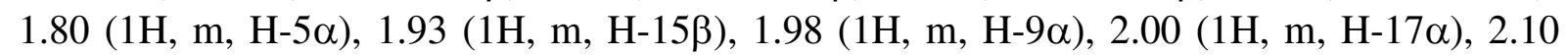
(1H, m, H-19ß), $2.15(1 \mathrm{H}, \mathrm{m}, \mathrm{H}-2 \alpha), 2.24\left(6 \mathrm{H}, \mathrm{bs}, \mathrm{NB}-\mathrm{CH}_{3}\right), 2.34(1 \mathrm{H}, \mathrm{d}, J=15.8 \mathrm{~Hz}, \mathrm{H}-$ $12 \beta), 2.56(1 \mathrm{H}, \mathrm{bd}, J=15.8 \mathrm{~Hz}, \mathrm{H}-12 \alpha), 2.60(1 \mathrm{H}, \mathrm{m}, \mathrm{H}-20), 2.79(1 \mathrm{H}, \mathrm{dd}, J=5.0,11.0 \mathrm{~Hz}$, H-3 $\alpha$ ), $3.28(1 \mathrm{H}, \mathrm{d}, J=14.9 \mathrm{~Hz}, \mathrm{H}-19 \alpha), 3.33(1 \mathrm{H}, \mathrm{d}, J=10.7 \mathrm{~Hz}, \mathrm{H}-29 \alpha), 3.87$ (1H, d, $J=$ $4.3 \mathrm{~Hz}, \mathrm{H}-1$ '), 3.99 (1H, d, $J=10.7 \mathrm{~Hz}, \mathrm{H}-29 \beta), 4.09$ (1H, dd, $J=7.2,7.3 \mathrm{~Hz}, \mathrm{H}-16 \beta), 5.51$ $(1 \mathrm{H}, \mathrm{m}, \mathrm{H}-1) ;{ }^{13} \mathrm{C} \mathrm{NMR}\left(300 \mathrm{MHz}, \mathrm{CDCl}_{3}\right) \delta 9.9\left(\mathrm{CH}_{3}, \mathrm{C}-30\right), 10.0\left(\mathrm{CH}_{3}, \mathrm{C}-21\right), 17.4\left(\mathrm{CH}_{3}\right.$, C-18), 17.8, $18.0\left(\mathrm{CH}_{3}, \mathrm{C}-3\right.$ ', C-4'), $18.8\left(\mathrm{CH}_{3}, \mathrm{C}-28\right), 24.3\left(\mathrm{CH}_{2}, \mathrm{C}-6\right), 29.7\left(\mathrm{CH}_{2}, \mathrm{C}-2\right), 32.7$ (CH, C-2'), $33.5\left(\mathrm{CH}_{2}, \mathrm{C}-7\right), 35.1$ (C, C-4), $37.4\left(\mathrm{CH}_{2}, \mathrm{C}-19\right), 42.4\left(\mathrm{CH}_{2}, \mathrm{C}-15\right), 46.2(\mathrm{C}, \mathrm{C}-$ 14), 47.0 (C, C-13), 48.6 (CH, C-5), 49.7 (CH, C-8), $50.0\left(\mathrm{CH}_{2}, \mathrm{C}-12\right), 50.4$ (CH, C-9), 55.4 (CH, C-17), $58.0(\mathrm{CH}, \mathrm{C}-3), 62.0(\mathrm{CH}, \mathrm{C}-20), 77.5\left(\mathrm{CH}_{2}, \mathrm{C}-29\right), 78.3(\mathrm{CH}, \mathrm{C}-16), 92.3(\mathrm{CH}$, C-1'), 120.1 (CH, C-1), 138.1 (C, C-10), 211.8 (C, C-11); ES-MS m/z $487.4[\mathrm{M}+\mathrm{H}]^{+}$(100); HRES-MS $m / z 487.3909$ (calcd for $\mathrm{C}_{30} \mathrm{H}_{51} \mathrm{~N}_{2} \mathrm{O}_{3}, 487.3900$ ). 


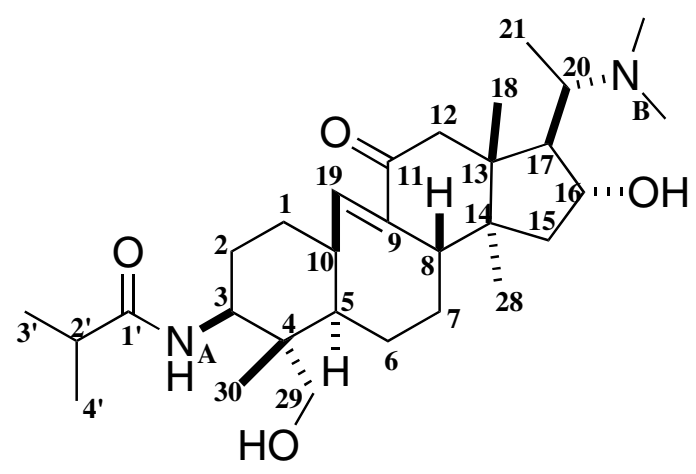

\section{$N$-[(2R,3S,3a $R, 6 \mathrm{a} S, 9 S, 10 S, 10 \mathrm{a} R, 12 \mathrm{a} S, 12 \mathrm{~b} S)-3$-[1-(dimethylamino)ethyl]-2-hydroxy-10 -(hydroxymethyl)-3a,10,12b-trimethyl-5-oxo-1,2,3,3a,4,5,6a,7,8,9,10,10a,11,12,12a,12b- hexadecahydrobenzo[4,5]cyclohepta[1,2-e]inden-9-yl]-2-methylpropanamide (13)}

To a stirred solution of compound $2(28 \mathrm{mg}, 0.05 \mathrm{mmol}, 1$ equiv) in $0.5 \mathrm{~mL}$ of freshly distilled dichloromethane was added boron trifluoride etherate $(2.6 \mathrm{~mL}, 0.06 \mathrm{mmol}, 1.2$ equiv) dropwise at $0{ }^{\circ} \mathrm{C}$. After stirring at $0{ }^{\circ} \mathrm{C}$ for 30 minutes, the mixture was stirred at room temperature for 2 days. The mixture was then made basic with $20 \mathrm{~mL}$ of a $10 \%$ sodium bicarbonate solution and extracted with dichloromethane $(3 \times 40 \mathrm{~mL})$. The organic layer was dried over $\mathrm{Na}_{2} \mathrm{SO}_{4}$ and filtered, and the solvent was evaporated to give a residue. A trituration of the crude product in diethyl ether afforded a colorless powder of $\mathbf{1 3}(24 \mathrm{mg}, 86 \%)$ whose spectroscopic characteristics were consistent with those of Herlem-Gaulier, D.; Kuong-HuuLainé, F.; Goutarel, R.; Magdeleine, M.J. Alcaloïdes stéroïdiques LXXII. Alcaloïdes du groupe des cylcloxobuxidines retirés du Buxus balearica Willd : $N$-3-isobutyryl cycloxobuxidine-F (baléabuxidine), N-3-isobutyryl cycloxobuxidine-H, N-3-benzoyl cycloxobuxidine-F et cycloxobuxoxazine-C Bull. Soc. Chim. Fr. 1968, 2, 763-773. mp 312 ${ }^{\circ} \mathrm{C} ;[\alpha]^{23}{ }_{\mathrm{D}}=-8\left(\mathrm{c} \mathrm{1.2}, \mathrm{CHCl}_{3}\right) ; \mathrm{IR}\left(\mathrm{CHCl}_{3}\right) \mathrm{U}_{\max }\left(\mathrm{cm}^{-1}\right): 3434,2935,1675,1651,1514,1462$, 1384, 1040; ${ }^{1} \mathrm{H}$ NMR $\left(300 \mathrm{MHz}, \mathrm{CDCl}_{3}\right) \delta 0.56(3 \mathrm{H}, \mathrm{s}, \mathrm{H}-30), 0.84(3 \mathrm{H}, \mathrm{s}, \mathrm{H}-18), 0.86(3 \mathrm{H}$, $\mathrm{d}, J=6.6 \mathrm{~Hz}, \mathrm{H}-21), 0.95(1 \mathrm{H}, \mathrm{m}, \mathrm{H}-6 \beta), 0.98(3 \mathrm{H}, \mathrm{s}, \mathrm{H}-28), 1.14,1.17(6 \mathrm{H}, 2 \mathrm{~d}, J=6.8 \mathrm{~Hz}$,

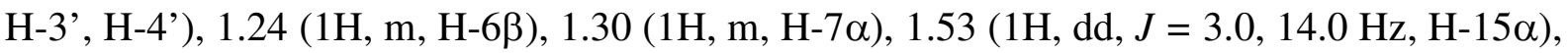
$1.58(1 \mathrm{H}, \mathrm{m}, \mathrm{H}-7 \beta), 1.61(1 \mathrm{H}, \mathrm{m}, \mathrm{H}-6 \alpha), 1.67(1 \mathrm{H}, \mathrm{m}, \mathrm{H}-2 \beta), 1.92(1 \mathrm{H}, \mathrm{dd}, J=7.5,10.7 \mathrm{~Hz}$, $\mathrm{H}-17 \alpha), 2.00(1 \mathrm{H}, \mathrm{m}, \mathrm{H}-15 \beta), 2.02(1 \mathrm{H}, \mathrm{m}, \mathrm{H}-8 \beta), 2.04(1 \mathrm{H}, \mathrm{m}, \mathrm{H}-2 \alpha), 2.14(1 \mathrm{H}, \mathrm{d}, J=18.8$ $\mathrm{Hz}, \mathrm{H}-12 \beta), 2.18(1 \mathrm{H}, \mathrm{m}, \mathrm{H}-10 \alpha), 2.24\left(6 \mathrm{H}, \mathrm{bs}, \mathrm{NB}-\mathrm{CH}_{3}\right), 2.38\left(1 \mathrm{H}, \mathrm{dq}, J=6.9 \mathrm{~Hz}, \mathrm{H}-2^{\prime}\right)$, $2.43(1 \mathrm{H}, \mathrm{m}, \mathrm{H}-1 \beta), 2.45$ (1H, m, H-5 $\alpha), 2.52(1 \mathrm{H}, \mathrm{bd}, J=18.8 \mathrm{~Hz}, \mathrm{H}-12 \alpha), 2.63(1 \mathrm{H}, \mathrm{dq}, J$ $=6.6,10.8 \mathrm{~Hz}, \mathrm{H}-20), 2.94(1 \mathrm{H}, \mathrm{dd}, J=9.8,12.5 \mathrm{~Hz}, \mathrm{H}-29 \mathrm{a}), 3.40(1 \mathrm{H}, \mathrm{dd}, J=10.7,11.7$ Hz, H-29b), 3.85 (1H, ddd, $J=2.0,8.3,9.0 \mathrm{~Hz}, \mathrm{H}-3 \alpha), 4.09$ (1H, ddd, $J=3.0,7.5,8.7 \mathrm{~Hz}$, $\mathrm{H}-16 \beta), 4.42(1 \mathrm{H}, \mathrm{d}, J=5.5 \mathrm{~Hz}, \mathrm{OH}), 5.52(1 \mathrm{H}, \mathrm{d}, J=8.3 \mathrm{~Hz}, \mathrm{NA} H), 6.54(1 \mathrm{H}, \mathrm{dd}, J=2.7$, $2.8 \mathrm{~Hz}, \mathrm{H}-19) ;{ }^{13} \mathrm{C}$ NMR $\left(300 \mathrm{MHz}, \mathrm{CDCl}_{3}\right) \delta 9.8\left(\mathrm{CH}_{3}, \mathrm{C}-21\right), 10.8\left(\mathrm{CH}_{3}, \mathrm{C}-30\right), 18.0\left(\mathrm{CH}_{3}\right.$, C-18), 19.4, 20.0 ( $\mathrm{CH}_{3}, \mathrm{C}-3$ ', C-4'), $20.0\left(\mathrm{CH}_{3}, \mathrm{C}-28\right), 27.5\left(\mathrm{CH}_{2}, \mathrm{C}-6\right), 29.7\left(\mathrm{CH}_{2}, \mathrm{C}-7\right), 31.8$ $\left(\mathrm{CH}_{2}, \mathrm{C}-2\right), 34.1\left(\mathrm{CH}_{2}, \mathrm{C}-1\right), 35.7\left(\mathrm{CH}, \mathrm{C}-2\right.$ ') $38.5(\mathrm{CH}, \mathrm{C}-10), 39.9(\mathrm{CH}, \mathrm{C}-5), 43.0\left(\mathrm{CH}_{2}\right.$, C-15), 43.2 (C, C-4), 43.6 (C, C-13), 45.8 (C, C-14), 47.3 (CH, C-8), $48.8\left(\mathrm{CH}_{2}, \mathrm{C}-12\right), 50.3$ $(\mathrm{CH}, \mathrm{C}-3), 56.0(\mathrm{CH}, \mathrm{C}-17), 62.0(\mathrm{CH}, \mathrm{C}-20), 63.7\left(\mathrm{CH}_{2}, \mathrm{C}-29\right), 78.0(\mathrm{CH}, \mathrm{C}-16), 141.8(\mathrm{C}$, C-9), 149.4 (CH, C-19), 178.5 (C, C-1'), 201.9 (C, C-11); ES-MS m/z $503.3[\mathrm{M}+\mathrm{H}]^{+}(100)$, 504.4 (90), 503.4 (10); HRES-MS $m / z 503.3843$ (calcd for $\mathrm{C}_{30} \mathrm{H}_{51} \mathrm{~N}_{2} \mathrm{O}_{4}, 503.3849$ ). 


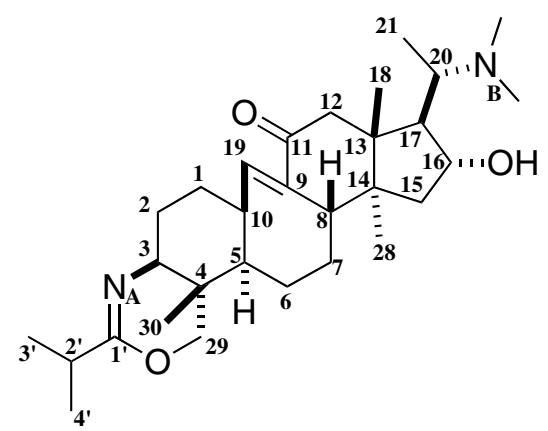

(4aS,6aS,9aR,10S,11R,12aS,12bS,14aR,14bS)-10-[1-(dimethylamino)ethyl]-11-hydroxy -3-isopropyl-9a,12a,14b-trimethyl-1,4a,5,6,6a,9,9a,10,11,12,12a,12b,13,14,14a,14bhexadecahydro-8H-indeno $\left[5^{\prime \prime}, 4^{\prime \prime}: 4^{\prime}, 5^{\prime}\right]$ cyclohepta[1',2':3,4]benzo[1,2-d][1,3] oxazin-8-one (14)

To a stirred solution of compound 13 (100 mg, $0.2 \mathrm{mmol}, 1$ equiv) in $2 \mathrm{~mL}$ of dichloromethane was added $25 \%$ tetraethylammonium hydroxide $(586 \mathrm{mg}, 1.0 \mathrm{mmol}, 5$ equiv) solution in methanol. The solvent was evaporated under reduced pressure. The dark red residue was heated at $300{ }^{\circ} \mathrm{C}$ under $0.03 \mathrm{mmHg}$ in a rotating distillation oven with four bulbs during 3 hours to afford a pale yellow powder of $14(60 \mathrm{mg}, 62 \%)$ : $\mathrm{mp} 279{ }^{\circ} \mathrm{C} ;[\alpha]^{24}{ }_{\mathrm{D}}=+14$ (c $\left.0.7, \mathrm{CHCl}_{3}\right)$; IR $\left(\mathrm{CHCl}_{3}\right) \cup_{\max }\left(\mathrm{cm}^{-1}\right): 3303,2932,1671,1600,1454,1382,1022 ;{ }^{1} \mathrm{H} \mathrm{NMR}$ $\left(300 \mathrm{MHz}, \mathrm{CDCl}_{3}\right) \delta 0.84(3 \mathrm{H}, \mathrm{s}, \mathrm{H}-18), 0.85(3 \mathrm{H}, \mathrm{d}, J=6.6 \mathrm{~Hz}, \mathrm{H}-21), 0.88(3 \mathrm{H}, \mathrm{s}, \mathrm{H}-30)$,

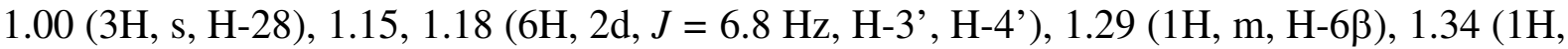

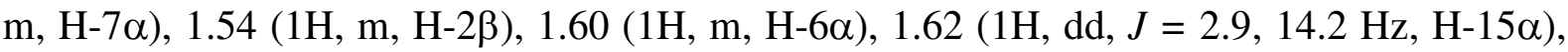
$1.82(1 \mathrm{H}, \mathrm{m}, \mathrm{H}-8 \beta), 1.84(1 \mathrm{H}, \mathrm{m}, \mathrm{H}-5 \alpha), 1.90(1 \mathrm{H}, \mathrm{m}, \mathrm{H}-7 \beta), 1.95(1 \mathrm{H}, \mathrm{m}, \mathrm{H}-15 \beta), 2.14(1 \mathrm{H}$, m, H-17 $), 2.31(1 \mathrm{H}, \mathrm{d}, J=18.5 \mathrm{~Hz}, \mathrm{H}-12 \beta), 2.32$ (6H, bs, NB-CH $), 2.33(1 \mathrm{H}, \mathrm{m}, \mathrm{H}-10 \alpha)$,

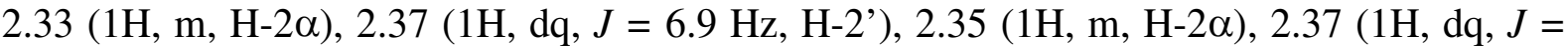
6.6, 11.2 Hz, H-20), $2.51(1 \mathrm{H}, \mathrm{d}, J=18.5 \mathrm{~Hz}, \mathrm{H}-12 \alpha), 2.80(1 \mathrm{H}, \mathrm{dd}, J=2.4,5.6 \mathrm{~Hz}, \mathrm{H}-3 \alpha)$, $3.75(1 \mathrm{H}, \mathrm{d}, J=10.2 \mathrm{~Hz}, \mathrm{H}-29 \alpha), 4.05(1 \mathrm{H}, \mathrm{d}, J=10.2 \mathrm{~Hz}, \mathrm{H}-29 \beta), 4.10$ (1H, ddd, $J=2.5$, 6.9, $9.7 \mathrm{~Hz}, \mathrm{H}-16 \beta), 6.45$ (1H, bs, H-19); ${ }^{13} \mathrm{C} \mathrm{NMR}\left(300 \mathrm{MHz}, \mathrm{CDCl}_{3}\right) \delta 9.9\left(\mathrm{CH}_{3}, \mathrm{C}-30\right)$, $\left.10.8\left(\mathrm{CH}_{3}, \mathrm{C}-21\right), 18.0\left(\mathrm{CH}_{3}, \mathrm{C}-18\right), 19.7,19.8\left(\mathrm{CH}_{3}, \mathrm{C}-3\right), \mathrm{C}-4\right), 20.1\left(\mathrm{CH}_{3}, \mathrm{C}-28\right), 27.6$ $\left(\mathrm{CH}_{2}, \mathrm{C}-6\right), 29.5\left(\mathrm{CH}_{2}, \mathrm{C}-7\right), 31.6\left(\mathrm{CH}_{2}, \mathrm{C}-2\right), 34.1\left(\mathrm{CH}_{2}, \mathrm{C}-1\right), 35.8(\mathrm{CH}, \mathrm{C}-2)$ ') $38.5(\mathrm{CH}, \mathrm{C}-$ 10), 39.9 (CH, C-5), $43.0\left(\mathrm{CH}_{2}, \mathrm{C}-15\right), 43.2$ (C, C-4), 43.6 (C, C-13), 45.8 (C, C-14), 47.3 $(\mathrm{CH}, \mathrm{C}-8), 48.8\left(\mathrm{CH}_{2}, \mathrm{C}-12\right), 50.3(\mathrm{CH}, \mathrm{C}-17), 56.1(\mathrm{CH}, \mathrm{C}-3), 62.0(\mathrm{CH}, \mathrm{C}-20), 76.6\left(\mathrm{CH}_{2}\right.$, C-29), 77.9 (CH, C-16), 142.5 (C, C-9), 149.4 (CH, C-19), 162.8 (C, C-1'), 202.4 (C, C-11); ES-MS $m / z 485.4[\mathrm{M}+\mathrm{H}]^{+}$(100), 486.4 (10), 503.4 (10); HRES-MS m/z 485.3702 (calcd for $\left.\mathrm{C}_{30} \mathrm{H}_{49} \mathrm{~N}_{2} \mathrm{O}_{3}, 485.3743\right)$. 


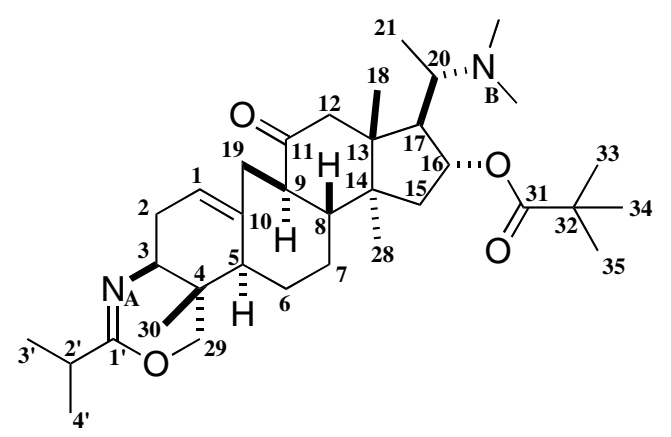

\section{$(4 \mathrm{a} S, 7 \mathrm{a} R, 9 \mathrm{a} R, 10 S, 11 R, 12 \mathrm{a} S, 12 \mathrm{~b} R, 14 \mathrm{a} R, 14 \mathrm{~b} S)$-10-[1-(dimethylamino)ethyl]-3-isopropyl- 9a,12a,14b-trimethyl-8-oxo-4a,5,7,7a,8,9,9a,10,11,12,12a,12b,13,14,14a,14b- hexadecahydro- $1 H$-indeno $\left[5^{\prime \prime}, 4^{\prime \prime}: 4^{\prime}, 5^{\prime}\right]$ cyclohepta $\left[1^{\prime}, 2^{\prime}: 3,4\right]$ benzo $[1,2-d][1,3]$ oxazin-11-yl acetate (15)}

To a stirred solution of compound 1 (50 mg, $0.1 \mathrm{mmol}, 1$ equiv) in $1 \mathrm{~mL}$ of pyridine was added pivaloyl chloride ( $13 \mu \mathrm{L}, 0.1 \mathrm{mmol}, 1$ equiv). After stirring at room temperature for 2 hours and 30 minutes, the mixture was then made basic with $30 \mathrm{~mL}$ of a $10 \%$ ammonia solution and extracted with dichloromethane $(3 \times 30 \mathrm{~mL})$. The organic layer was dried over $\mathrm{Na}_{2} \mathrm{SO}_{4}$ and filtered, and the solvent was evaporated to afford a colorless powder of 15 (45 $\mathrm{mg}, 77 \%)$ : IR $\left(\mathrm{CHCl}_{3}\right) \cup_{\max }\left(\mathrm{cm}^{-1}\right): 2966,2931,1720,1698,1671,1459,1364,1158 ;{ }^{1} \mathrm{H} \mathrm{NMR}$ $\left(500 \mathrm{MHz}, \mathrm{CDCl}_{3}\right) \delta 0.65(3 \mathrm{H}, \mathrm{s}, \mathrm{H}-18), 0.68(3 \mathrm{H}, \mathrm{s}, \mathrm{H}-30), 0.78(3 \mathrm{H}, \mathrm{d}, J=6.9 \mathrm{~Hz}, \mathrm{H}-21)$, 1.04, 1.06 (6H, 2d, J = 6.9 Hz, H-3', H-4'), 1.12 (9H, s, H-33, H-34, H-35), 1.19 (3H, s, H-

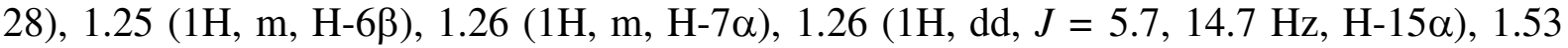
$(1 \mathrm{H}, \mathrm{dd}, J=6.9,12.0 \mathrm{~Hz}, \mathrm{H}-6 \alpha), 1.62(1 \mathrm{H}, \mathrm{ddd}, J=3.8,10.4,10.7 \mathrm{~Hz}, \mathrm{H}-2 \beta), 1.72(1 \mathrm{H}, \mathrm{m}$, $\mathrm{H}-8 \beta), 1.79(1 \mathrm{H}, \mathrm{m}, \mathrm{H}-7 \beta), 1.81(1 \mathrm{H}, \mathrm{m}, \mathrm{H}-5 \alpha), 1.87$ (1H, dd, $J=9.0,9.5 \mathrm{~Hz}, \mathrm{H}-9 \alpha), 1.98$ $(1 \mathrm{H}, \mathrm{m}, \mathrm{H}-15 \beta), 2.02(1 \mathrm{H}, \mathrm{m}, \mathrm{H}-19 \beta), 2.06\left(6 \mathrm{H}, \mathrm{s}, \mathrm{NB}-C_{3}\right), 2.20(1 \mathrm{H}, \mathrm{dd}, J=5.6,11.4 \mathrm{~Hz}$, $\mathrm{H}-17 \alpha), 2.28(1 \mathrm{H}, \mathrm{m}, \mathrm{H}-2 \alpha), 2.34(1 \mathrm{H}, \mathrm{dq}, J=6.9 \mathrm{~Hz}, \mathrm{H}-2$ '), $2.36(1 \mathrm{H}, \mathrm{d}, J=15.8 \mathrm{~Hz}, \mathrm{H}-$ $12 \beta), 2.38(1 \mathrm{H}, \mathrm{m}, \mathrm{H}-20), 2.52(1 \mathrm{H}, \mathrm{bd}, J=15.8 \mathrm{~Hz}, \mathrm{H}-12 \alpha), 3.16(1 \mathrm{H}, \mathrm{dd}, J=6.3,11.7 \mathrm{~Hz}$,

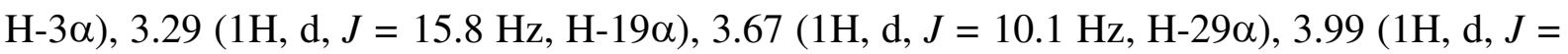
$10.1 \mathrm{~Hz}, \mathrm{H}-29 \beta), 4.98(1 \mathrm{H}, \mathrm{dd}, J=5.7,13.2 \mathrm{~Hz}, \mathrm{H}-16 \beta), 5.53(1 \mathrm{H}, \mathrm{m}, \mathrm{H}-1) ;{ }^{13} \mathrm{C}$ NMR $(125$ $\left.\mathrm{MHz}, \mathrm{CDCl}_{3}\right) \delta 9.62\left(\mathrm{CH}_{3}, \mathrm{C}-30\right), 10.03\left(\mathrm{CH}_{3}, \mathrm{C}-21\right), 17.84\left(\mathrm{CH}_{3}, \mathrm{C}-18\right), 19.77\left(\mathrm{CH}_{3}, \mathrm{C}-3{ }^{\prime}\right.$, C-4'), $19.84\left(\mathrm{CH}_{3}, \mathrm{C}-28\right), 24.61\left(\mathrm{CH}_{2}, \mathrm{C}-6\right), 27.17\left(\mathrm{CH}_{3}, \mathrm{C}-33, \mathrm{C}-34, \mathrm{C}-35\right), 30.73\left(\mathrm{CH}_{2}, \mathrm{C}-\right.$ 2), 33.20 (C, C-4), $33.52\left(\mathrm{CH}_{2}, \mathrm{C}-7\right), 34.31\left(\mathrm{CH}, \mathrm{C}-2\right.$ ') $37.11\left(\mathrm{CH}_{2}, \mathrm{C}-19\right), 38.54(\mathrm{C}, \mathrm{C}-32)$, $40.64\left(\mathrm{CH}_{3}, \mathrm{NB}-\mathrm{CH}_{3}\right), 43.26\left(\mathrm{CH}_{2}, \mathrm{C}-15\right), 46.41(\mathrm{C}, \mathrm{C}-14), 47.11(\mathrm{C}, \mathrm{C}-13), 49.56(\mathrm{CH}, \mathrm{C}-5)$, $49.82(\mathrm{CH}, \mathrm{C}-8), 50.31\left(\mathrm{CH}_{2}, \mathrm{C}-12\right), 50.32(\mathrm{CH}, \mathrm{C}-9), 54.07(\mathrm{CH}, \mathrm{C}-17), 55.65(\mathrm{CH}, \mathrm{C}-3)$, $59.61(\mathrm{CH}, \mathrm{C}-20), 74.74\left(\mathrm{CH}_{2}, \mathrm{C}-29\right), 78.94(\mathrm{CH}, \mathrm{C}-16), 121.87$ (CH, C-1), 137.08 (C, C-10), 162.70 (C, C-1'), 177.63 (C, C-31), 211.23 (C, C-11); ES-MS m/z $569.4[\mathrm{M}+\mathrm{H}]^{+}$(100), 570.4 (30), 485.4 (5), 285.2 (10); HRES-MS $m / z 569.4301$ (calcd for $\mathrm{C}_{35} \mathrm{H}_{57} \mathrm{~N}_{2} \mathrm{O}_{4}, 569.4318$ ). 


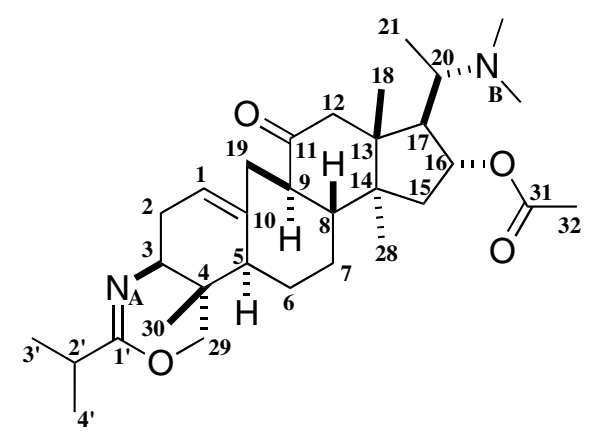

$(4 \mathrm{a} S, 7 \mathrm{a} R, 9 \mathrm{a} R, 10 S, 11 R, 12 \mathrm{a} S, 12 \mathrm{~b} R, 14 \mathrm{a} R, 14 \mathrm{~b} S)-10$-[1-(dimethylamino)ethyl]-3-isopropyl9a,12a,14b-trimethyl-8-oxo-4a,5,7,7a,8,9,9a,10,11,12,12a,12b,13,14,14a,14bhexadecahydro-1H-indeno $\left[5^{\prime \prime}, 4^{\prime \prime}: 4^{\prime}, 5^{\prime}\right]$ cyclohepta[1',2':3,4]benzo[1,2-d][1,3] oxazin11-yl 2,2-dimethylpropanoate (16)

To a stirred solution of compound 1 (50 mg, $0.1 \mathrm{mmol}, 1$ equiv) in $1 \mathrm{~mL}$ of pyridine was added acetyl chloride ( $8 \mu \mathrm{L}, 0.1 \mathrm{mmol}, 1$ equiv). After stirring at room temperature for 2 hours and 30 minutes, the mixture was then made basic with $30 \mathrm{~mL}$ of a $10 \%$ ammonia solution and extracted with dichloromethane $(3 \times 30 \mathrm{~mL})$. The organic layer was dried over $\mathrm{Na}_{2} \mathrm{SO}_{4}$ and filtered, and the solvent was co-evaporated with 1,2-dichloroethane to afford a pale yellow powder of 16 (37 mg, 70\%): $\mathrm{mp} 207{ }^{\circ} \mathrm{C}$; IR $\left(\mathrm{CHCl}_{3}\right) \cup_{\max }\left(\mathrm{cm}^{-1}\right): 2965,2932$, $1729,1696,1670,1457,1375,1245,1027 ;{ }^{1} \mathrm{H}$ NMR $\left(300 \mathrm{MHz}, \mathrm{CDCl}_{3}\right) \delta 0.68(3 \mathrm{H}, \mathrm{s}, \mathrm{H}-18)$, 0.73 (3H, s, H-30), 0.80 (3H, d, $J=6.2 \mathrm{~Hz}, \mathrm{H}-21), 1.09$ (6H, 2d, $J=6.9 \mathrm{~Hz}, \mathrm{H}-3$ ', H-4'), 1.18 (3H, s, H-28), $1.28(1 \mathrm{H}, \mathrm{m}, \mathrm{H}-6 \beta), 1.30(1 \mathrm{H}, \mathrm{m}, \mathrm{H}-7 \alpha), 1.41(1 \mathrm{H}, \mathrm{bd}, J=14.3 \mathrm{~Hz}, \mathrm{H}-15 \alpha)$, $1.59(1 \mathrm{H}, \mathrm{m}, \mathrm{H}-6 \alpha), 1.66(1 \mathrm{H}, \mathrm{m}, \mathrm{H}-8 \beta), 1.80(1 \mathrm{H}, \mathrm{m}, \mathrm{H}-2 \beta), 1.82(1 \mathrm{H}, \mathrm{m}, \mathrm{H}-5 \alpha), 1.83(1 \mathrm{H}$,

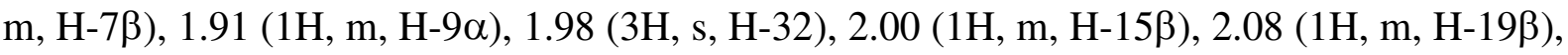
$2.12\left(6 \mathrm{H}, \mathrm{s}, \mathrm{NB}-\mathrm{CH}_{3}\right), 2.23(1 \mathrm{H}, \mathrm{dd}, J=6.0,11.0 \mathrm{~Hz}, \mathrm{H}-17 \alpha), 2.31(1 \mathrm{H}, \mathrm{m}, \mathrm{H}-2 \alpha), 2.40(1 \mathrm{H}$, $\mathrm{d}, J=15.6 \mathrm{~Hz}, \mathrm{H}-12 \beta), 2.42(1 \mathrm{H}, \mathrm{dq}, J=6.9 \mathrm{~Hz}, \mathrm{H}-2$ ' $), 2.45(1 \mathrm{H}, \mathrm{dq}, J=6.2,11.0 \mathrm{~Hz}, \mathrm{H}-$ 20), 2.56 (1H, bd, $J=15.6 \mathrm{~Hz}, \mathrm{H}-12 \alpha), 3.20(1 \mathrm{H}, \mathrm{dd}, J=6.0,11.4 \mathrm{~Hz}, \mathrm{H}-3 \alpha), 3.31(1 \mathrm{H}, \mathrm{d}, J$ $=15.3 \mathrm{~Hz}, \mathrm{H}-19 \alpha), 3.73(1 \mathrm{H}, \mathrm{d}, J=10.2 \mathrm{~Hz}, \mathrm{H}-29 \alpha), 4.04(1 \mathrm{H}, \mathrm{d}, J=10.2 \mathrm{~Hz}, \mathrm{H}-29 \beta), 5.10$ $(1 \mathrm{H}, \mathrm{dd}, J=6.9,9.7 \mathrm{~Hz}, \mathrm{H}-16 \beta), 5.58(1 \mathrm{H}, \mathrm{m}, \mathrm{H}-1) ;{ }^{13} \mathrm{C} \mathrm{NMR}\left(75,5 \mathrm{MHz}, \mathrm{CDCl}_{3}\right) \delta 9.6$ $\left.\left(\mathrm{CH}_{3}, \mathrm{C}-30\right), 9.9\left(\mathrm{CH}_{3}, \mathrm{C}-21\right), 17.8\left(\mathrm{CH}_{3}, \mathrm{C}-18\right), 19.7,19.8\left(\mathrm{CH}_{3}, \mathrm{C}-3\right), \mathrm{C}-4\right), 21.1\left(\mathrm{CH}_{3}, \mathrm{C}-\right.$ 28, C-32), $24.6\left(\mathrm{CH}_{2}, \mathrm{C}-6\right), 30.6\left(\mathrm{CH}_{2}, \mathrm{C}-2\right), 33.2$ (C, C-4), $33.4\left(\mathrm{CH}_{2}, \mathrm{C}-7\right), 34.2(\mathrm{CH}, \mathrm{C}-2$ '), $37.1\left(\mathrm{CH}_{2}, \mathrm{C}-19\right), 40.1\left(\mathrm{CH}_{3}, \mathrm{NB}-\mathrm{CH}_{3}\right), 42.6\left(\mathrm{CH}_{2}, \mathrm{C}-15\right), 46.1(\mathrm{C}, \mathrm{C}-14), 47.2(\mathrm{C}, \mathrm{C}-13)$, $49.3(\mathrm{CH}, \mathrm{C}-5), 49.5(\mathrm{CH}, \mathrm{C}-8), 49.8(\mathrm{C}, \mathrm{C}-9), 50.2\left(\mathrm{CH}_{2}, \mathrm{C}-12\right), 54.2(\mathrm{CH}, \mathrm{C}-17), 55.5(\mathrm{CH}$, C-3), $59.5(\mathrm{CH}, \mathrm{C}-20), 74.8\left(\mathrm{CH}_{2}, \mathrm{C}-29\right), 78.6(\mathrm{CH}, \mathrm{C}-16), 121.8(\mathrm{CH}, \mathrm{C}-1), 137.0(\mathrm{C}, \mathrm{C}-10)$, 163.1 (C, C-1'), 170.5 (C, C-31), 211.2 (C, C-11); ES-MS m/z $527.4[\mathrm{M}+\mathrm{H}]^{+}(100), 485.3$ (50); HRES-MS $m / z 527.3851$ (calcd for $\mathrm{C}_{32} \mathrm{H}_{51} \mathrm{~N}_{2} \mathrm{O}_{4}, 527.3840$ ). 


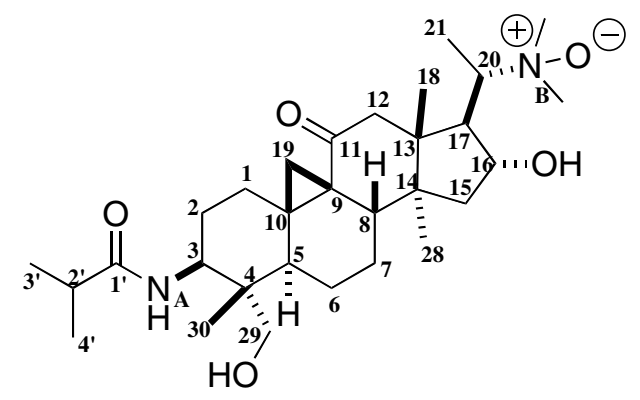

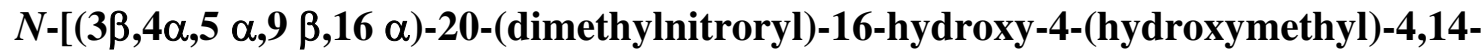 dimethyl-11-oxo-9,19-cyclopregnan-3-yl]-2-methylpropanamide (17)}

To a stirred solution of compound 2 (202 mg, $0.40 \mathrm{mmol}, 1.0$ equiv) in $6 \mathrm{~mL}$ of dichloromethane was added 3-chloroperbenzoic acid (95 mg, $0.55 \mathrm{mmol}, 1.4$ equiv). After stirring at room temperature for 30 minutes, the mixture was then, made basic with $40 \mathrm{~mL}$ of a $10 \%$ ammonia solution and extracted with dichloromethane $(3 \times 40 \mathrm{~mL})$. Emulsifications were broken with ultrasonic waves. The organic layer was dried over $\mathrm{Na}_{2} \mathrm{SO}_{4}$ and filtered, and the solvent was evaporated under reduced pressure. A trituration of the residue in diethyl ether afforded a colorless powder of $17(165 \mathrm{mg}, 79 \%)$ : $\mathrm{mp} 205^{\circ} \mathrm{C}$; IR $\left(\mathrm{CHCl}_{3}\right) \mathrm{v}_{\max }\left(\mathrm{cm}^{-1}\right)$ : 3434, 2967, 2935, 1655, 1513, 1455, 1382, 1049; ${ }^{1} \mathrm{H}$ NMR (300 MHz, $\left.\mathrm{CDCl}_{3}\right) \delta 0.57(3 \mathrm{H}, \mathrm{s}, \mathrm{H}-30$ ), 0.94 (3H, s, H-18), 1.09 (1H, d, $J=3.7$ Hz, H-19 $), 1.17,1.19$ (6H, 2d, $J=6.4$ Hz, H-3', H4'), 1.24 (3H, s, H-28), 1.34 (3H, d, $J=6.8 \mathrm{~Hz}, \mathrm{H}-21), 0.86(1 \mathrm{H}, \mathrm{m}, \mathrm{H}-6 \beta), 1.25(1 \mathrm{H}, \mathrm{m}, \mathrm{H}-$ $1 \alpha), 1.55(1 \mathrm{H}, \mathrm{d}, J=3.7 \mathrm{~Hz}, \mathrm{H}-19 \beta), 1.54(1 \mathrm{H}, \mathrm{m}, \mathrm{H}-7 \alpha), 1.58(1 \mathrm{H}, \mathrm{m}, \mathrm{H}-7 \beta), 1.60(1 \mathrm{H}, \mathrm{m}$, $\mathrm{H}-2 \beta), 1.70(1 \mathrm{H}, \mathrm{m}, \mathrm{H}-15 \alpha), 1.71(1 \mathrm{H}, \mathrm{m}, \mathrm{H}-2 \alpha), 1.72(1 \mathrm{H}, \mathrm{m}, \mathrm{H}-6 \alpha), 2.01(1 \mathrm{H}, \mathrm{m}, \mathrm{H}-5 \alpha)$,

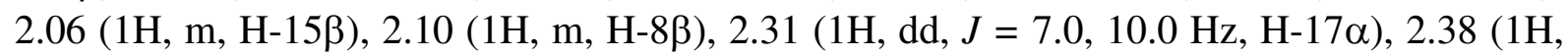
dq, $J=6.4$ Hz, H-2'), $2.42(1 \mathrm{H}, \mathrm{m}, \mathrm{H}-1 \beta), 2.43(1 \mathrm{H}, \mathrm{d}, J=15.8 \mathrm{~Hz}, \mathrm{H}-12 \alpha), 2.61(1 \mathrm{H}, \mathrm{d}, J=$ $15.8 \mathrm{~Hz}, \mathrm{H}-12 \beta), 2.97(1 \mathrm{H}, \mathrm{d}, J=12.8 \mathrm{~Hz}, \mathrm{H}-29 \mathrm{a}), 3.12,3.32\left(6 \mathrm{H}, 2 \mathrm{~s}, \mathrm{NB}-\mathrm{CH}_{3}\right), 3.35(1 \mathrm{H}$, m, H-29b), 3.44 (1H, m, H-20), 4.00 (1H, ddd, $J=4.0,8.9,12.3 \mathrm{~Hz}, \mathrm{H}-3 \alpha), 4.59$ (1H, ddd, $J$ $=2.3,7.0,9.9 \mathrm{~Hz}, \mathrm{H}-16 \beta), 5.37(1 \mathrm{H}, \mathrm{d}, J=9.2 \mathrm{~Hz}, \mathrm{NA} H) ;{ }^{13} \mathrm{C} \mathrm{NMR}\left(300 \mathrm{MHz}, \mathrm{CDCl}_{3}\right) \delta$ $11.2\left(\mathrm{CH}_{3}, \mathrm{C}-30\right), 17.5\left(\mathrm{CH}_{3}, \mathrm{C}-21\right), 17.6\left(\mathrm{CH}_{3}, \mathrm{C}-18\right), 18.4\left(\mathrm{CH}_{2}, \mathrm{C}-6\right), 19.4,20.2\left(\mathrm{CH}_{3}, \mathrm{C}-\right.$ 3', C-4'), $20.5\left(\mathrm{CH}_{3}, \mathrm{C}-28\right), 24.3\left(\mathrm{CH}_{2}, \mathrm{C}-7\right), 27.6,27.9\left(\mathrm{CH}_{2}, \mathrm{C}-2, \mathrm{C}-1\right), 31.2\left(\mathrm{CH}_{2}, \mathrm{C}-19\right)$, 34.0 (C, C-9), 35.8 (CH, C-2'), 38.1 (C, C-10), 41.2 (CH, C-5), $41.3(\mathrm{CH}, \mathrm{C}-8), 43.1\left(\mathrm{CH}_{2}, \mathrm{C}-\right.$ 15), 44.6 (C, C-4), 46.7 (C, C-13), $47.9(\mathrm{C}, \mathrm{C}-14), 50.6(\mathrm{CH}, \mathrm{C}-3), 51.3\left(\mathrm{CH}_{3}, \mathrm{NB}-\mathrm{CH} 3\right), 52.6$ $\left(\mathrm{CH}_{2}, \mathrm{C}-12\right), 56.4(\mathrm{CH}, \mathrm{C}-17), 61.3\left(\mathrm{CH}_{3}, \mathrm{NB}-\mathrm{CH} 3\right), 64.1\left(\mathrm{CH}_{2}, \mathrm{C}-29\right), 75.1(\mathrm{CH}, \mathrm{C}-16), 78.7$ (CH, C-20), 178.5 (C, C-1'), 211.0 (C, C-11); ES-MS m/z $519.3[\mathrm{M}+\mathrm{H}]^{+}(100), 520.3$ (10), 480.3 (20), 440.3 (30), 370.3 (20), 353.2 (10), 341.2 (25), 323.2 (25); HRES-MS m/z 519.3770 (calcd for $\mathrm{C}_{30} \mathrm{H}_{51} \mathrm{~N}_{2} \mathrm{O}_{5}, 519.3770$ ). 


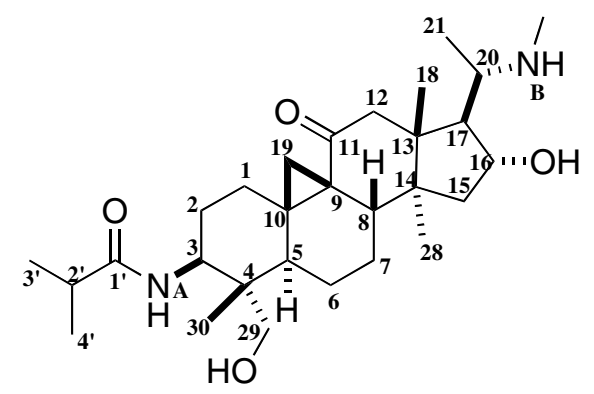

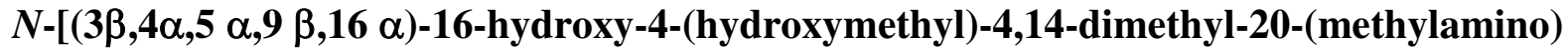 -11-oxo-9,19-cyclopregnan-3-yl]-2-methylpropanamide (18)}

To a stirred solution of compound 17 (29.5 mg, $0.06 \mathrm{mmol}, 1$ equiv) in $3 \mathrm{~mL}$ of methanol was added heptahydrated iron (II) sulfate (32 mg, $0.11 \mathrm{mmol}, 2$ equiv) and hexahydrated iron (III) chloride (15 mg, $0.06 \mathrm{mmol}, 1$ equiv). After stirring at room temperature for 1 hour and 30 minutes, the solvent was then evaporated under reduced pressure. The residue dissolved in $40 \mathrm{~mL}$ of dichloromethane was made basic with $20 \mathrm{~mL}$ of a $10 \%$ sodium bicarbonate solution to get the iron carbonates precipitated. The mixture was extracted with dichloromethane $(3 \times 30$ $\mathrm{mL})$. Emulsifications were broken with ultrasonic waves. The organic layer was acidified with a $1 \mathrm{M}$ buffer solution of glacial acetic acid-sodium acetate $(30 \mathrm{~mL})$. After stirring for 15 minutes, the mixture was then, extracted with dichloromethane $(5 \times 40 \mathrm{~mL})$, washed with 20 $\mathrm{mL}$ of brine, dried over $\mathrm{Na}_{2} \mathrm{SO}_{4}$ and filtered, and the solvent was evaporated. A trituration of the residue in diethyl ether afforded a colorless powder of $\mathbf{1 8}(21 \mathrm{mg}, 76 \%): \mathrm{mp} 268{ }^{\circ} \mathrm{C}$; $[\alpha]^{21}=+78\left(\mathrm{c} 1.1, \mathrm{CHCl}_{3}\right) ; \mathrm{IR}\left(\mathrm{CHCl}_{3}\right) \mathrm{v}_{\max }\left(\mathrm{cm}^{-1}\right): 3434,2967,2935,1655,1514,1456$, 1381, 1093; ${ }^{1} \mathrm{H}$ NMR (300 MHz, $\left.\mathrm{CDCl}_{3}\right) \delta 0.84(3 \mathrm{H}, \mathrm{s}, \mathrm{H}-18), 0.93(3 \mathrm{H}, \mathrm{s}, \mathrm{H}-30), 1.00(1 \mathrm{H}$,

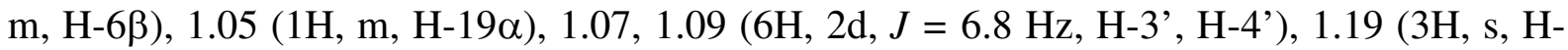
28), $1.21(3 \mathrm{H}, \mathrm{d}, J=7.3 \mathrm{~Hz}, \mathrm{H}-21), 1.25(1 \mathrm{H}, \mathrm{m}, \mathrm{H}-1 \alpha), 1.55(1 \mathrm{H}, \mathrm{m}, \mathrm{H}-7 \alpha), 1.57(1 \mathrm{H}, \mathrm{m}, \mathrm{H}-$

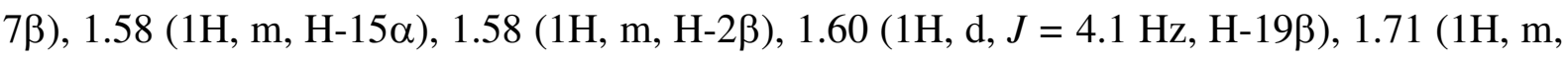

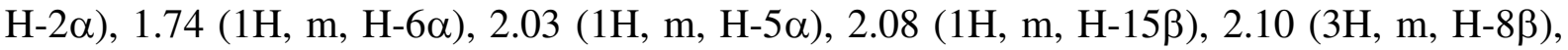
$2.45\left(3 \mathrm{H}, \mathrm{s}, \mathrm{HNB}-\mathrm{CH}_{3}\right), 2.13(1 \mathrm{H}, \mathrm{m}, \mathrm{H}-17 \alpha), 2.34(1 \mathrm{H}, \mathrm{d}, J=17.1 \mathrm{~Hz}, \mathrm{H}-12 \beta), 2.35(1 \mathrm{H}, \mathrm{m}$, H-20), $2.38(1 \mathrm{H}, \mathrm{dq}, J=6.8 \mathrm{~Hz}, \mathrm{H}-2$ '), $2.43(1 \mathrm{H}, \mathrm{m}, \mathrm{H}-1 \beta), 2.57(1 \mathrm{H}, \mathrm{d}, J=17.1 \mathrm{~Hz}, \mathrm{H}-$ $12 \alpha), 4.33(1 \mathrm{H}, \mathrm{ddd}, J=3.9,9.0,12.0 \mathrm{~Hz}, \mathrm{H}-3 \alpha), 5.13(1 \mathrm{H}, \mathrm{dd}, J=5.8,8.3 \mathrm{~Hz}, \mathrm{H}-16 \beta), 5.28$ $(1 \mathrm{H}, \mathrm{d}, J=8.8 \mathrm{~Hz}, \mathrm{NAH}), 9.41(1 \mathrm{H}, \mathrm{s}, \mathrm{H}-29) ;{ }^{13} \mathrm{C} \mathrm{NMR}\left(300 \mathrm{MHz}, \mathrm{CDCl}_{3}\right) \delta 9.9\left(\mathrm{CH}_{3}, \mathrm{C}-\right.$ 21), $11.2\left(\mathrm{CH}_{3}, \mathrm{C}-30\right), 18.0\left(\mathrm{CH}_{3}, \mathrm{C}-18\right), 18.4\left(\mathrm{CH}_{2}, \mathrm{C}-6\right), 19.5,20.1\left(\mathrm{CH}_{3}, \mathrm{C}-3\right.$ ', C-4'), 20.6 $\left(\mathrm{CH}_{3}, \mathrm{C}-28\right), 24.3\left(\mathrm{CH}_{2}, \mathrm{C}-7\right), 27.6,27.7\left(\mathrm{CH}_{2}, \mathrm{C}-2, \mathrm{C}-1\right), 29.7\left(\mathrm{CH}_{2}, \mathrm{C}-19\right), 34.4(\mathrm{C}, \mathrm{C}-9)$, $35.8\left(\mathrm{CH}, \mathrm{C}-2\right.$ '), 37.8 (C, C-10), $41.2(\mathrm{CH}, \mathrm{C}-5), 41.5(\mathrm{CH}, \mathrm{C}-8), 42.8\left(\mathrm{CH}_{2}, \mathrm{C}-15\right), 44.5(\mathrm{C}$, C-13), 44.6 (C, C-4), 47.1 (C, C-14), 50.7 (CH, C-3), $51.4\left(\mathrm{CH}_{2}, \mathrm{C}-12\right), 58.6(\mathrm{CH}, \mathrm{C}-17), 62.0$ (CH, C-20), $64.2\left(\mathrm{CH}_{2}, \mathrm{C}-29\right), 77.9$ (CH, C-16), 178.5 (C, C-1'), 211.0 (C, C-11); ES-MS m/z $489.3[\mathrm{M}+\mathrm{H}]^{+}(100), 503.4$ (40); HRES-MS $m / z 489.3688$ (calcd for $\mathrm{C}_{29} \mathrm{H}_{49} \mathrm{~N}_{2} \mathrm{O}_{4}, 489.3692$ ). 


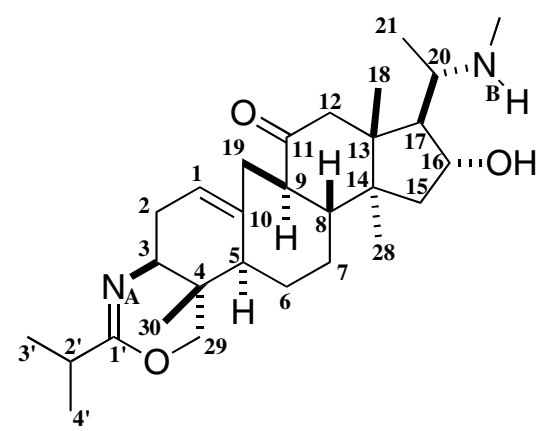

$4 \mathrm{a} S, 7 \mathrm{a} R, 9 \mathrm{a} R, 10 S, 11 R, 12 \mathrm{a} S, 12 \mathrm{~b} R, 14 \mathrm{a} R, 14 \mathrm{~b} S)$-11-hydroxy-3-isopropyl-9a,12a,14btrimethyl-10-[1-(methylamino)ethyl]-1,4a,5,7,7a,9,9a,10,11,12,12a,12b,13,14,14a,14b hexadecahydro-8H-indeno[5",4'":4',5']cyclohepta[1',2':3,4]benzo[1,2-d][1,3] oxazin-8-one (19)

Compound 18 (100 mg, $0.20 \mathrm{mmol}, 1$ equiv) was heated at $273{ }^{\circ} \mathrm{C}$ under $0.09 \mathrm{mmHg}$ in a rotating distillation oven with four bulbs. A product sublimated in 3 hours. To a solution of the sublimate in $2 \mathrm{~mL}$ of dichloromethane was added $25 \%$ tetraethylammonium hydroxide (600 mg, $1.02 \mathrm{mmol}, 5$ equiv) solution in methanol. The solvent was evaporated under reduced pressure. The dark red residue was heated at $273{ }^{\circ} \mathrm{C}$ under $0.09 \mathrm{mmHg}$ in a rotating distillation oven with four bulbs to afford a sublimated pale yellow powder of 19 (56 mg, $60 \%)$ in 3 hours: IR $\left(\mathrm{CHCl}_{3}\right) v_{\max }\left(\mathrm{cm}^{-1}\right): 3367,2964,2931,1697,1667,1455,1383,1147$, 1074; ${ }^{1} \mathrm{H}$ NMR $\left(300 \mathrm{MHz}, \mathrm{CDCl}_{3}\right) \delta 0.68(3 \mathrm{H}, \mathrm{s}, \mathrm{H}-18), 0.74(3 \mathrm{H}, \mathrm{s}, \mathrm{H}-30), 1.11(6 \mathrm{H}, 2 \mathrm{~d}, J=$ 6.9 Hz, H-3', H-4'), 1.21 (3H, s, H-28), 1.22 (3H, d, J = $7.3 \mathrm{~Hz}, \mathrm{H}-21), 1.27$ (1H, m, H-6ß), $1.33(1 \mathrm{H}, \mathrm{m}, \mathrm{H}-7 \alpha), 1.50(1 \mathrm{H}, \mathrm{bd}, J=13.8 \mathrm{~Hz}, \mathrm{H}-15 \alpha), 1.61(1 \mathrm{H}, \mathrm{m}, \mathrm{H}-6 \alpha), 1.65(1 \mathrm{H}, \mathrm{m}, \mathrm{H}-$

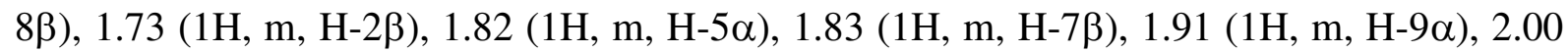

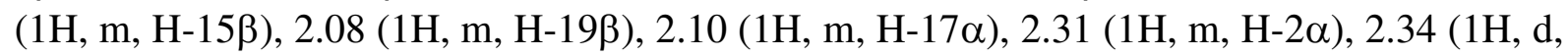
$J=15.1 \mathrm{~Hz}, \mathrm{H}-12 \beta), 2.36$ (1H, m, H-20), 2.40 (1H, dq, $J=6.9 \mathrm{~Hz}, \mathrm{H}-2$ '), 2.43 (3H, s, HNB$\left.C_{3}\right), 2.54(1 \mathrm{H}, \mathrm{bd}, J=15.1 \mathrm{~Hz}, \mathrm{H}-12 \alpha), 3.23(1 \mathrm{H}, \mathrm{dd}, J=6.0,11.5 \mathrm{~Hz}, \mathrm{H}-3 \alpha), 3.32(1 \mathrm{H}, \mathrm{d}$, $J=15.4 \mathrm{~Hz}, \mathrm{H}-19 \alpha), 3.78(1 \mathrm{H}, \mathrm{d}, J=10.2 \mathrm{~Hz}, \mathrm{H}-29 \alpha), 4.06(1 \mathrm{H}, \mathrm{d}, J=10.2 \mathrm{~Hz}, \mathrm{H}-29 \beta)$, $4.07(1 \mathrm{H}, \mathrm{m}, \mathrm{H}-16 \beta), 5.59(1 \mathrm{H}, \mathrm{m}, \mathrm{H}-1) ;{ }^{13} \mathrm{C} \mathrm{NMR}\left(75.5 \mathrm{MHz}, \mathrm{CDCl}_{3}\right) \delta 9.7\left(\mathrm{CH}_{3}, \mathrm{C}-30\right)$, $17.8\left(\mathrm{CH}_{3}, \mathrm{C}-18\right), 18.7\left(\mathrm{CH}_{3}, \mathrm{C}-21\right), 18.7\left(\mathrm{CH}_{3}, \mathrm{C}-28\right), 19.8,19.9\left(\mathrm{CH}_{3}, \mathrm{C}-3{ }^{\prime}, \mathrm{C}-4{ }^{\prime}\right), 24.7$ $\left(\mathrm{CH}_{2}, \mathrm{C}-6\right), 30.8\left(\mathrm{CH}_{2}, \mathrm{C}-2\right), 33.2(\mathrm{C}, \mathrm{C}-4), 33.5\left(\mathrm{CH}_{2}, \mathrm{C}-7\right), 34.4(\mathrm{CH}, \mathrm{C}-2$ ') $) 37.3\left(\mathrm{CH}_{2}, \mathrm{C}-\right.$ 19), $42.5\left(\mathrm{CH}_{2}, \mathrm{C}-15\right), 47.0$ (C, C-14), 48.1 (C, C-13), 49.5 (CH, C-5), 49.7 (CH, C-8), 50.0 $\left(\mathrm{CH}_{2}, \mathrm{C}-12\right), 50.3(\mathrm{CH}, \mathrm{C}-9), 50.3\left(\mathrm{CH}_{3}, \mathrm{HNB}-\mathrm{CH} 3\right), 55.6(\mathrm{CH}, \mathrm{C}-3), 58.6(\mathrm{CH}, \mathrm{C}-17), 60.4$ (CH, C-20), $74.8\left(\mathrm{CH}_{2}, \mathrm{C}-29\right), 78.2(\mathrm{CH}, \mathrm{C}-16), 121.8(\mathrm{CH}, \mathrm{C}-1), 137.2$ (C, C-10), 162.7 (C, C-1'), 211.2 (C, C-11); ES-MS m/z 471.4 [M+H] ${ }^{+}$(100), 499.4 (90), 438.3 (20); HRES-MS $m / z, 471.3590$ (calcd for $\mathrm{C}_{29} \mathrm{H}_{47} \mathrm{~N}_{2} \mathrm{O}_{3}, 471.3587$ 


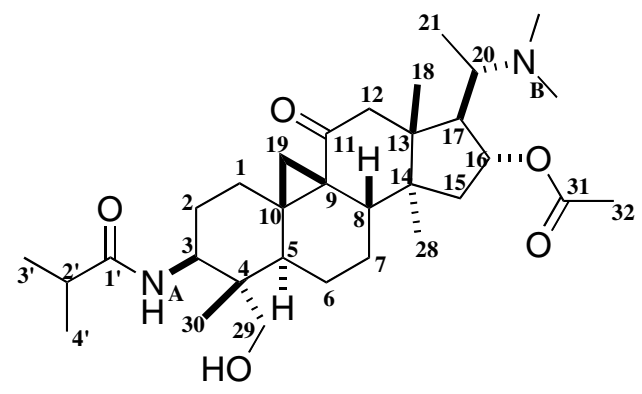

(3 $\alpha, 4 \alpha, 5 \alpha, 8 \alpha, 10 \alpha, 14 \beta, 16 \beta, 17 \alpha, 20 S)$-20-(dimethylamino)-4-(hydroxymethyl)-3(isobutyrylamino)-4,14-dimethyl-11-oxo-9,19-cyclopregnan-16-yl acetate (20a)

To a solution of compound $2(1007 \mathrm{mg}, 2.0 \mathrm{mmol}, 1$ equiv) in $100 \mathrm{~mL}$ of freshly distilled dichloromethane and $30 \mathrm{~mL}$ of dried pyridine was added acetic anhydride $(568 \mu \mathrm{L}, 6.0 \mathrm{mmol}$, 3.0 equiv). After stirring at room temperature for 2 days, the solvent was co-evaporated with 1,2-dichloroethane under reduced pressure. The residue was purified by column chromatography on silica with dichloromethane-methanol $(9: 1)$ as eluent followed by crystallisation in acetone to afford a colorless powder of 20a $(820 \mathrm{mg}, 75 \%): \mathrm{mp} 283{ }^{\circ} \mathrm{C}$; $[\alpha]^{23}{ }_{\mathrm{D}}=+36\left(\mathrm{c} 0.9 \mathrm{CHCl}_{3}\right) ; \mathrm{IR}\left(\mathrm{CHCl}_{3}\right) \mathrm{v}_{\max }\left(\mathrm{cm}^{-1}\right): 3276,2931,1729,1667,1632,1555$, 1454, 1372, 1243, 1044; ${ }^{1} \mathrm{H}$ NMR $\left(300 \mathrm{MHz}, \mathrm{CDCl}_{3}\right) \delta 0.56(3 \mathrm{H}, \mathrm{s}, \mathrm{H}-30), 0.84(3 \mathrm{H}, \mathrm{m}, \mathrm{H}-$

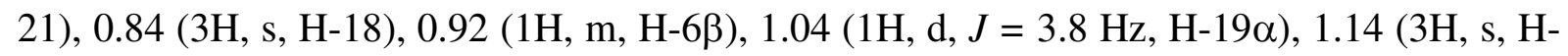

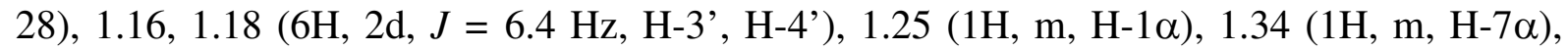
$1.41(1 \mathrm{H}, \mathrm{m}, \mathrm{H}-15 \alpha), 1.46(1 \mathrm{H}, \mathrm{m}, \mathrm{H}-7 \beta), 1.50(1 \mathrm{H}, \mathrm{m}, \mathrm{H}-2 \beta), 1.56(1 \mathrm{H}, \mathrm{d}, J=3.8 \mathrm{~Hz}, \mathrm{H}-$

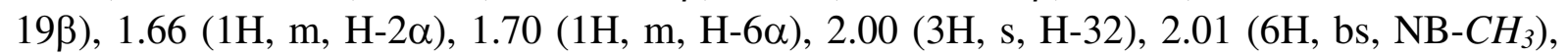

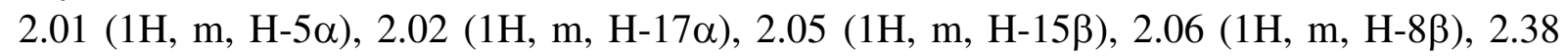
$(1 \mathrm{H}, \mathrm{m}, \mathrm{H}-20), 2.38(1 \mathrm{H}, \mathrm{d}, J=17.2 \mathrm{~Hz}, \mathrm{H}-12 \beta), 2.39(3 \mathrm{H}, \mathrm{s}, \mathrm{H}-32), 2.30(1 \mathrm{H}, \mathrm{dq}, J=7.0$ Hz, H-2'), $2.43(1 \mathrm{H}, \mathrm{m}, \mathrm{H}-1 \beta), 2.54(1 \mathrm{H}, \mathrm{d}, J=17.2 \mathrm{~Hz}, \mathrm{H}-12 \alpha), 2.96(1 \mathrm{H}, \mathrm{dd}, J=4.9,12.8$ Hz, H-29a), 3.33 (1H, d, $J=10.9,12.8 \mathrm{~Hz}, \mathrm{H}-29 \mathrm{~b}), 3.98$ (1H, ddd, $J=4.3,8.6,12.5 \mathrm{~Hz}, \mathrm{H}-$ $3 \alpha), 4.40(1 \mathrm{H}, \mathrm{dd}, J=4.9,10.9 \mathrm{~Hz}, \mathrm{OH}), 5.13(1 \mathrm{H}, \mathrm{m}, \mathrm{H}-16 \beta), 5.41(1 \mathrm{H}, \mathrm{d}, J=9.0 \mathrm{~Hz}$, $\mathrm{NAH}) ;{ }^{13} \mathrm{C}$ NMR $\left(300 \mathrm{MHz}, \mathrm{CDCl}_{3}\right) \delta 11.2\left(\mathrm{CH}_{3}, \mathrm{C}-30, \mathrm{C}-21\right), 17.8\left(\mathrm{CH}_{3}, \mathrm{C}-18\right), 18.4\left(\mathrm{CH}_{2}\right.$, C-6), 19.4, $19.6\left(\mathrm{CH}_{3}, \mathrm{C}-3\right.$ ', C-4'), $20.2\left(\mathrm{CH}_{3}, \mathrm{C}-28\right), 21.2\left(\mathrm{CH}_{3}, \mathrm{C}-32\right), 24.3\left(\mathrm{CH}_{2}, \mathrm{C}-7\right)$, 27.6, $27.7\left(\mathrm{CH}_{2}, \mathrm{C}-2, \mathrm{C}-1\right), 30.7\left(\mathrm{CH}_{2}, \mathrm{C}-19\right), 34.0$ (C, C-9), $35.8\left(\mathrm{CH}, \mathrm{C}-2\right.$ '), $40.6\left(\mathrm{CH}_{3}\right.$, NB$\left.\mathrm{CH}_{3}\right), 37.9(\mathrm{C}, \mathrm{C}-10), 41.2(\mathrm{CH}, \mathrm{C}-5), 41.4(\mathrm{CH}, \mathrm{C}-8), 43.1\left(\mathrm{CH}_{2}, \mathrm{C}-15\right), 44.6(\mathrm{C}, \mathrm{C}-13), 47.4$ (C, C-14), $50.7(\mathrm{CH}, \mathrm{C}-3), 51.9\left(\mathrm{CH}_{2}, \mathrm{C}-12\right), 54.9(\mathrm{CH}, \mathrm{C}-17), 59.6(\mathrm{CH}, \mathrm{C}-20), 64.1\left(\mathrm{CH}_{2}\right.$, C-29), 78.8 (CH, C-16), 170.7 (C, C-31), 178.5 (C, C-1'), 211.5 (C, C-11); ES-MS m/z 545.4 $[\mathrm{M}+\mathrm{H}]^{+}(100), 546.4$ (5), 503.4 (60); HRES-MS m/z 545.3960 (calcd for $\mathrm{C}_{32} \mathrm{H}_{53} \mathrm{~N}_{2} \mathrm{O}_{5}$, 545.3954). 


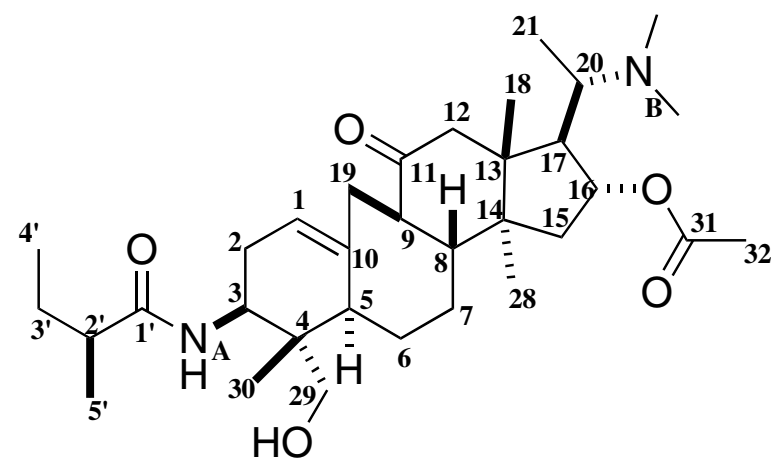

(3 $\beta, 4 \alpha, 5 \alpha, 9 \beta, 16 \alpha)-20$-(dimethylamino)-4-(hydroxymethyl)-4,14-dimethyl-3-\{[(2 S)-2methylbutanoyl]amino\}-11-oxo-9,19-cyclopregnan-16-yl acetate (20b)

To a solution of compound $\mathbf{7 d}$ (735 mg, $1.42 \mathrm{mmol}, 1.0$ equiv) in $71 \mathrm{~mL}$ of freshly distilled dichloromethane and $21 \mathrm{~mL}$ of dried pyridine was added acetic anhydride $(202 \mu \mathrm{L}, 2.13$ mmol, 1.5 equiv). After stirring at room temperature for 16 hours, the solvent was coevaporated with 1,2-dichloroethane under reduced pressure. The residue was then made basic with $30 \mathrm{~mL}$ of a $10 \%$ ammonia solution and extracted with dichloromethane $(3 \times 20 \mathrm{~mL})$. The organic layer was dried over $\mathrm{Na}_{2} \mathrm{SO}_{4}$ and filtered, and the solvent was evaporated under reduced pressure. The residue was crystallized with acetone to afford a colorless powder of $20 \mathrm{~b}(169 \mathrm{mg})$. The mother liquor was then purified by column chromatography on alumina with dichloromethane-methanol (9:1) as eluent followed by crystallisation in acetone to afford a colorless powder of $\mathbf{2 0 b}(378 \mathrm{mg}, 90 \%)$ : $\mathrm{mp} 270{ }^{\circ} \mathrm{C}$; $[\alpha]^{23}{ }_{\mathrm{D}}=+53\left(\mathrm{c}^{1.2} \mathrm{CHCl}_{3}\right)$; IR $\left(\mathrm{CHCl}_{3}\right) \cup_{\max }\left(\mathrm{cm}^{-1}\right): 3238,2962-2928,1731,1698,1632,1552,1454,1377,1243,1034 ;{ }^{1} \mathrm{H}$ NMR (300 MHz, $\left.\mathrm{CDCl}_{3}\right) \delta 0.47(3 \mathrm{H}, \mathrm{s}, \mathrm{H}-30), 0.70(3 \mathrm{H}, \mathrm{s}, \mathrm{H}-18), 0.82(3 \mathrm{H}, \mathrm{d}, J=6.2 \mathrm{~Hz}, \mathrm{H}-$ 21), 0.95 (3H, t, $\left.J=7.5 \mathrm{~Hz}, \mathrm{H}-4^{\prime}\right), 1.16$ (3H, d, $\left.J=6.8 \mathrm{~Hz}, \mathrm{H}-5^{\prime}\right), 1.19$ (1H, m, H-6ß), 1.22 (3H, s, H-28), 1.42 (1H, m, H-7 $), 1.43$ (1H, bd, $J=14.0 \mathrm{~Hz}, \mathrm{H}-15 \alpha), 1.49$ (1H, m, H-3a'), $1.67(1 \mathrm{H}, \mathrm{m}, \mathrm{H}-3 \mathrm{~b}$ '), $1.82(1 \mathrm{H}, \mathrm{m}, \mathrm{H}-7 \beta), 1.86(1 \mathrm{H}, \mathrm{m}, \mathrm{H}-6 \alpha), 1.90(1 \mathrm{H}, \mathrm{m}, \mathrm{H}-9 \alpha), 2.00(1 \mathrm{H}$,

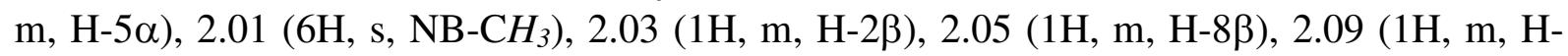

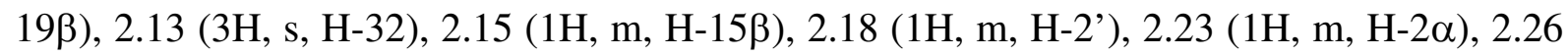
$(1 \mathrm{H}, \mathrm{dd}, J=6.1,11.0 \mathrm{~Hz}, \mathrm{H}-17 \alpha), 2.41(1 \mathrm{H}, \mathrm{d}, J=15.6 \mathrm{~Hz}, \mathrm{H}-12 \beta), 2.45(1 \mathrm{H}, \mathrm{m}, \mathrm{H}-20)$, $2.61(1 \mathrm{H}, \mathrm{d}, J=15.6 \mathrm{~Hz}, \mathrm{H}-12 \alpha), 3.16(1 \mathrm{H}, \mathrm{dd}, J=4.5,12.4 \mathrm{~Hz}, \mathrm{H}-29 \mathrm{a}), 3.35(1 \mathrm{H}, \mathrm{d}, J=$ $14.3 \mathrm{~Hz}, \mathrm{H}-19 \alpha), 3.40(1 \mathrm{H}, \mathrm{d}, J=10.9,12.4 \mathrm{~Hz}, \mathrm{H}-29 \mathrm{~b}), 4.08$ (1H, ddd, $J=6.1,9.0,10.9 \mathrm{~Hz}$, $\mathrm{H}-3 \alpha), 4.66(1 \mathrm{H}, \mathrm{dd}, J=4.5,10.9 \mathrm{~Hz}, \mathrm{OH}), 5.12(1 \mathrm{H}, \mathrm{dd}, J=6.1,8.0 \mathrm{~Hz}, \mathrm{H}-16 \beta), 5.39(1 \mathrm{H}$, $\mathrm{d}, J=8.9 \mathrm{~Hz}, \mathrm{NAH}), 5.48(1 \mathrm{H}, \mathrm{s}, \mathrm{H}-1) ;{ }^{13} \mathrm{C} \mathrm{NMR}\left(300 \mathrm{MHz}, \mathrm{CDCl}_{3}\right) \delta 9.9\left(\mathrm{CH}_{3}, \mathrm{C}-30\right), 10.0$ $\left(\mathrm{CH}_{3}, \mathrm{C}-21\right), 12.1\left(\mathrm{CH}_{3}, \mathrm{C}-4\right.$ ') $17.6\left(\mathrm{CH}_{3}, \mathrm{C}-5\right), 17.9\left(\mathrm{CH}_{3}, \mathrm{C}-18\right), 18.0\left(\mathrm{CH}_{3}, \mathrm{C}-28\right), 21.2$ $\left(\mathrm{CH}_{3}, \mathrm{C}-32\right), 25.0\left(\mathrm{CH}_{2}, \mathrm{C}-6\right), 27.5\left(\mathrm{CH}_{2}, \mathrm{C}-3\right), 30.1\left(\mathrm{CH}_{2}, \mathrm{C}-2\right), 33.4\left(\mathrm{CH}_{2}, \mathrm{C}-7\right), 37.5\left(\mathrm{CH}_{2}\right.$, C-19), $\left.40.1\left(\mathrm{CH}_{3}, \mathrm{NB}-\mathrm{CH}_{3}\right), 42.0(\mathrm{C}, \mathrm{C}-4), 42.7\left(\mathrm{CH}_{2}, \mathrm{C}-15\right), 43.3(\mathrm{CH}, \mathrm{C}-2)\right), 43.5(\mathrm{CH}, \mathrm{C}-$ 5), 46.2 (C, C-13), 47.3 (C, C-14), 48.0 (CH, C-3), 49.7 (CH, C-8), 49.9 (CH, C-9), 50.3 $\left(\mathrm{CH}_{2}, \mathrm{C}-12\right), 54.2(\mathrm{CH}, \mathrm{C}-17), 59.6(\mathrm{CH}, \mathrm{C}-20), 63.8\left(\mathrm{CH}_{2}, \mathrm{C}-29\right), 78.7(\mathrm{CH}, \mathrm{C}-16), 117.9$ (CH, C-1), 139.9 (C, C-10), 170.6 (C, C-31), 178.4 (C, C-1'), 211.6 (C, C-11); ES-MS m/z $559.4[\mathrm{M}+\mathrm{H}]^{+}(100), 560.4$ (15); HRES-MS m/z 559.4150 (calcd for $\mathrm{C}_{33} \mathrm{H}_{55} \mathrm{~N}_{2} \mathrm{O}_{5}, 559.4111$ ). 


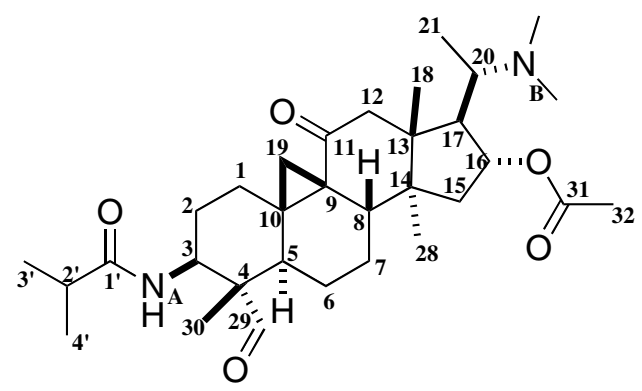

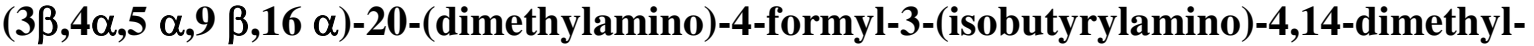 11-oxo-9,19-cyclopregnan-16-yl acetate (21a)}

To a stirred solution of compound $\mathbf{2 0 a}$ (200 $\mathrm{mg}, 0.36 \mathrm{mmol}, 1$ equiv) in $20 \mathrm{~mL}$ of dichloromethane was added Dess-Martin periodinane (209 mg, $0.47 \mathrm{mmol}, 1.3$ equiv). After stirring at room temperature for 16 hours, the mixture was then made basic with $40 \mathrm{~mL}$ of a $10 \%$ ammonia solution and extracted with dichloromethane $(3 \times 40 \mathrm{~mL})$. The organic layer was dried over $\mathrm{Na}_{2} \mathrm{SO}_{4}$ and filtered, and the solvent was evaporated under reduced pressure. The residue was purified by flash chromatography on silica with dichloromethane-methanol (95:5) as eluent to afford a colorless powder of $21 \mathrm{a}(127 \mathrm{mg}, 64 \%)$ : $\mathrm{mp} 255{ }^{\circ} \mathrm{C}$; IR $\left(\mathrm{CHCl}_{3}\right)$ $U_{\max }\left(\mathrm{cm}^{-1}\right): 3397,2962,2773,1728,1656,1517,1448,1375,1241,1096 ;{ }^{1} \mathrm{H}$ NMR (300 $\left.\mathrm{MHz}, \mathrm{CDCl}_{3}\right) \delta 0.82(3 \mathrm{H}, \mathrm{d}, J=6.6 \mathrm{~Hz}, \mathrm{H}-21), 0.84(3 \mathrm{H}, \mathrm{s}, \mathrm{H}-18), 0.93(3 \mathrm{H}, \mathrm{s}, \mathrm{H}-30), 1.00$

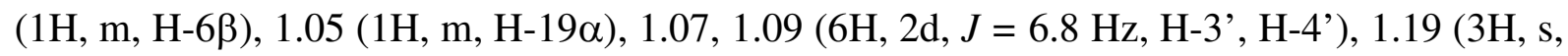
$\mathrm{H}-28), 1.27(1 \mathrm{H}, \mathrm{m}, \mathrm{H}-1 \alpha), 1.37(1 \mathrm{H}, \mathrm{m}, \mathrm{H}-7 \alpha), 1.43(1 \mathrm{H}, \mathrm{bd}, J=14.1 \mathrm{~Hz}, \mathrm{H}-15 \alpha), 1.44(1 \mathrm{H}$, m, H-7 $\beta), 1.52(1 \mathrm{H}, \mathrm{m}, \mathrm{H}-2 \beta), 1.60(1 \mathrm{H}, \mathrm{d}, J=4.1 \mathrm{~Hz}, \mathrm{H}-19 \beta), 1.78(1 \mathrm{H}, \mathrm{m}, \mathrm{H}-2 \alpha), 1.82$

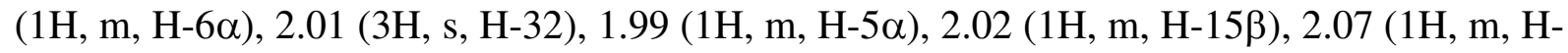
$8 \beta), 2.15\left(6 \mathrm{H}, \mathrm{bs}, \mathrm{NB}-\mathrm{CH}_{3}\right), 2.23(1 \mathrm{H}, \mathrm{m}, \mathrm{H}-17 \alpha), 2.25(1 \mathrm{H}, \mathrm{dq}, J=6.8 \mathrm{~Hz}, \mathrm{H}-2$ ') $2.34(1 \mathrm{H}$, $\mathrm{d}, J=17.1 \mathrm{~Hz}, \mathrm{H}-12 \beta), 2.40(1 \mathrm{H}, \mathrm{m}, \mathrm{H}-1 \beta), 2.46(1 \mathrm{H}, \mathrm{m}, \mathrm{H}-20), 2.57(1 \mathrm{H}, \mathrm{d}, J=17.1 \mathrm{~Hz}, \mathrm{H}-$ $12 \alpha), 4.33$ (1H, ddd, $J=3.9,9.0,12.0 \mathrm{~Hz}, \mathrm{H}-3 \alpha), 5.13(1 \mathrm{H}, \mathrm{dd}, J=5.8,8.3 \mathrm{~Hz}, \mathrm{H}-16 \beta), 5.28$ $(1 \mathrm{H}, \mathrm{d}, J=8.8 \mathrm{~Hz}, \mathrm{NAH}), 9.41(1 \mathrm{H}, \mathrm{s}, \mathrm{H}-29) ;{ }^{13} \mathrm{C} \mathrm{NMR}\left(300 \mathrm{MHz}, \mathrm{CDCl}_{3}\right) \delta 7.7\left(\mathrm{CH}_{3}, \mathrm{C}-\right.$ 21), $9.9\left(\mathrm{CH}_{3}, \mathrm{C}-30\right), 17.9\left(\mathrm{CH}_{3}, \mathrm{C}-18\right), 19.3,19.4\left(\mathrm{CH}_{3}, \mathrm{C}-3\right.$ ', C-4'), $19.7\left(\mathrm{CH}_{3}, \mathrm{C}-32\right), 21.0$ $\left(\mathrm{CH}_{2}, \mathrm{C}-6\right), 21.2\left(\mathrm{CH}_{3}, \mathrm{C}-28\right), 24.0\left(\mathrm{CH}_{2}, \mathrm{C}-7\right), 27.4\left(\mathrm{CH}_{2}, \mathrm{C}-2\right), 27.5\left(\mathrm{CH}_{2}, \mathrm{C}-1\right), 29.5\left(\mathrm{CH}_{2}\right.$, C-19), 33.6 (C, C-9), 35.6 (CH, C-2'), 35.9 (C, C-10), $40.2\left(\mathrm{CH}_{3}, \mathrm{NB}-\mathrm{CH}_{3}\right), 41.2(\mathrm{CH}, \mathrm{C}-5)$, $41.8(\mathrm{CH}, \mathrm{C}-8), 42.9\left(\mathrm{CH}_{2}, \mathrm{C}-15\right), 44.5(\mathrm{C}, \mathrm{C}-13), 47.4(\mathrm{C}, \mathrm{C}-14), 49.5(\mathrm{CH}, \mathrm{C}-3), 51.8\left(\mathrm{CH}_{2}\right.$, C-12), 55.1 (CH, C-17), 56.9 (C, C-4), 59.5 (CH, C-20), 78.7 (CH, C-16), 170.7 (C, C-31), 176.3 (C, C-1'), 203.3 (C, C-29), 210.56 (C, C-11); ES-MS m/z 543.4 [M+H] ${ }^{+}$(100), 501.4 (5); HRES-MS m/z 543.3784 (calcd for $\mathrm{C}_{32} \mathrm{H}_{50} \mathrm{~N}_{2} \mathrm{O}_{5}, 543.3798$ ). 


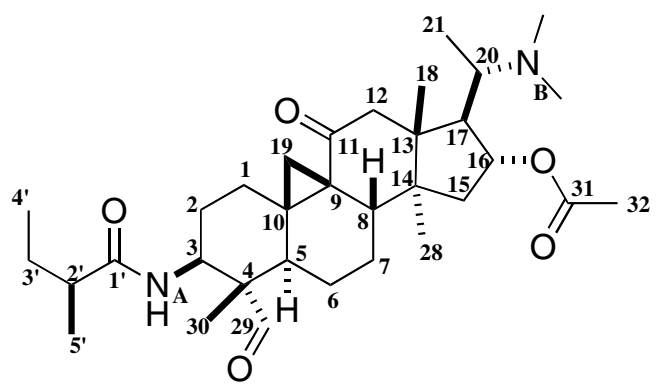

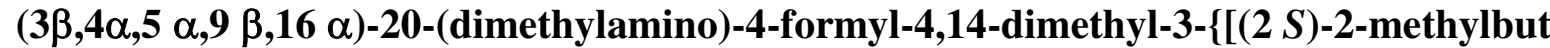 anoyl]amino\}-11-oxo-9,19-cyclopregnan-16-yl acetate (21b)}

To a solution of compound $7 \mathbf{d}$ (496 mg, $0.96 \mathrm{mmol}, 1.0$ equiv) in $48 \mathrm{~mL}$ of freshly distilled dichloromethane and $14 \mathrm{~mL}$ of dried pyridine was added acetic anhydride $(136 \mu \mathrm{L}, 1.44$ mmol, 1.5 equiv). After stirring at room temperature for 16 hours, the solvent was coevaporated with 1,2-dichloroethane under reduced pressure. The residue was then made basic with $40 \mathrm{~mL}$ of a $10 \%$ ammonia solution and extracted with dichloromethane $(3 \times 30 \mathrm{~mL})$. The organic layer was dried over $\mathrm{Na}_{2} \mathrm{SO}_{4}$ and filtered, and the solvent was evaporated under reduced pressure. To a stirred solution of crude residue (519 $\mathrm{mg}, 0.93 \mathrm{mmol}, 1.0$ equiv) in 30 $\mathrm{mL}$ of dichloromethane was added Dess-Martin periodinane $(511 \mathrm{mg}, 1.21 \mathrm{mmol}, 1.3 \mathrm{equiv})$. After stirring at room temperature for 1 hour, the mixture was then made basic with $40 \mathrm{~mL}$ of a $10 \%$ ammonia solution and extracted with dichloromethane $(3 \times 30 \mathrm{~mL})$. The organic layer was dried over $\mathrm{Na}_{2} \mathrm{SO}_{4}$ and filtered, and the solvent was evaporated under reduced pressure. The residue was purified by column chromatography on alumina with dichloromethanemethanol (95:5) as eluent followed by a trituration in acetone to afford a colorless powder of $21 b$ (500 mg, 91\% in 2 steps): $\mathrm{mp} 265{ }^{\circ} \mathrm{C}$; IR $\left(\mathrm{CHCl}_{3}\right) \mathrm{v}_{\max }\left(\mathrm{cm}^{-1}\right): 3254,2975-2926,2856$, $1718,1666,1638,1538,1447,1376,1255,1017 ;{ }^{1} \mathrm{H}$ NMR $\left(300 \mathrm{MHz}, \mathrm{CDCl}_{3}\right) \delta 0.81(3 \mathrm{H}, \mathrm{d}$, $J=6.4 \mathrm{~Hz}, \mathrm{H}-21), 0.83$ (3H, s, H-18), 0.84 (3H, t, $\left.J=7.3 \mathrm{~Hz}, \mathrm{H}-4^{\prime}\right), 0.92$ (3H, s, H-30), 1.06 $(1 \mathrm{H}, \mathrm{m}, \mathrm{H}-6 \beta), 1.06(3 \mathrm{H}, \mathrm{d}, J=6.8 \mathrm{~Hz}, \mathrm{H}-5$ ') $1.07(1 \mathrm{H}, \mathrm{d}, J=3.6 \mathrm{~Hz}, \mathrm{H}-19 \alpha), 1.18$ (3H, s,

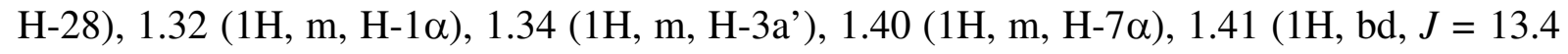

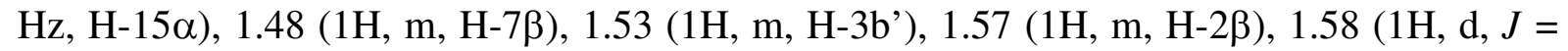

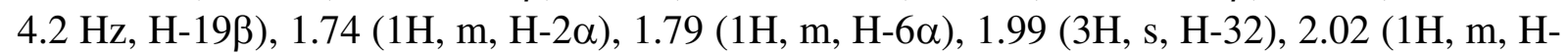
$5 \alpha), 2.04(1 \mathrm{H}, \mathrm{m}, \mathrm{H}-15 \beta), 2.10(1 \mathrm{H}, \mathrm{m}, \mathrm{H}-8 \beta), 2.13\left(6 \mathrm{H}, \mathrm{s}, \mathrm{NB}-\mathrm{CH}_{3}\right), 2.22(1 \mathrm{H}, \mathrm{dd}, J=6.2$,

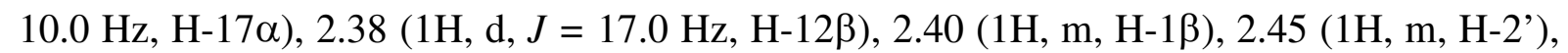
$2.47(1 \mathrm{H}, \mathrm{m}, \mathrm{H}-20), 2.56(1 \mathrm{H}, \mathrm{d}, J=17.1 \mathrm{~Hz}, \mathrm{H}-12 \alpha), 4.34(1 \mathrm{H}, \mathrm{ddd}, J=4.1,8.9,12.4 \mathrm{~Hz}$, $\mathrm{H}-3 \alpha), 5.11(1 \mathrm{H}, \mathrm{dd}, J=6.2,8.0 \mathrm{~Hz}, \mathrm{H}-16 \beta), 5.28(1 \mathrm{H}, \mathrm{d}, J=9.1 \mathrm{~Hz}, \mathrm{NA} H), 9.41(1 \mathrm{H}, \mathrm{s}, \mathrm{H}-$ $29) ;{ }^{13} \mathrm{C}$ NMR $\left(300 \mathrm{MHz}, \mathrm{CDCl}_{3}\right) \delta 7.8\left(\mathrm{CH}_{3}, \mathrm{C}-30\right), 10.0\left(\mathrm{CH}_{3}, \mathrm{C}-21\right), 11.8\left(\mathrm{CH}_{3}, \mathrm{C}-4{ }^{\prime}\right)$, $17.4\left(\mathrm{CH}_{3}, \mathrm{C}-5\right), 18.0\left(\mathrm{CH}_{3}, \mathrm{C}-18\right), 19.5\left(\mathrm{CH}_{3}, \mathrm{C}-28\right), 21.1\left(\mathrm{CH}_{2}, \mathrm{C}-6\right), 21.2\left(\mathrm{CH}_{3}, \mathrm{C}-31\right)$, $24.2\left(\mathrm{CH}_{2}, \mathrm{C}-7\right), 27.5\left(\mathrm{CH}_{2}, \mathrm{C}-2\right), 27.6\left(\mathrm{CH}_{2}, \mathrm{C}-3\right.$ ') $27.6\left(\mathrm{CH}_{2}, \mathrm{C}-1\right), 29.6\left(\mathrm{CH}_{2}, \mathrm{C}-19\right), 33.7$ (C, C-9), 36.0 (C, C-10), $40.4\left(\mathrm{CH}_{3}, \mathrm{NB}-\mathrm{CH}_{3}\right), 41.3\left(\mathrm{CH}, \mathrm{C}-2\right.$ '), $41.8(\mathrm{CH}, \mathrm{C}-5), 43.0\left(\mathrm{CH}_{2}\right.$, C-15), 43.3 (CH, C-8), 44.6 (C, C-13), 47.5 (C, C-14), $50.0(\mathrm{CH}, \mathrm{C}-3), 51.9\left(\mathrm{CH}_{2}, \mathrm{C}-12\right), 55.3$ (CH, C-17), 56.9 (C, C-4), 59.6 (CH, C-20), 78.9 (CH, C-16), 170.7 (C, C-31), 175.9 (C, C1'), 203.5 (C, C-29), 211.6 (C, C-11); ES-MS m/z $557.4[\mathrm{M}+\mathrm{H}]^{+}$(100), 558.4 (15), 515.4 (20); HRES-MS $m / z, 557.3948$ (calcd for $\mathrm{C}_{33} \mathrm{H}_{53} \mathrm{~N}_{2} \mathrm{O}_{5}, 557.3954$ ). 


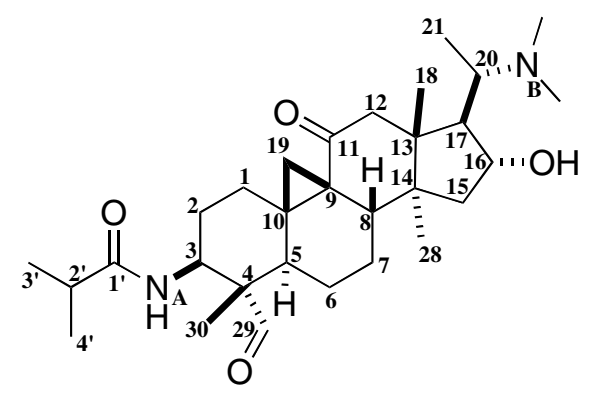

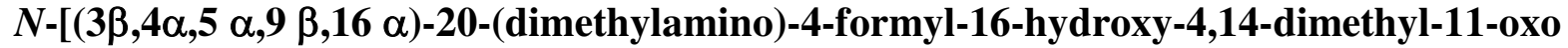 -9,19-cyclopregnan-3-yl]-2-methylpropanamide (22a)}

To a stirred solution of compound $21 \mathrm{a}$ (1000 mg, $1.84 \mathrm{mmol}, 1$ equiv) in $23 \mathrm{~mL}$ of methanol was added a suspension of sodium carbonate (1953 mg, $18.42 \mathrm{mmol}, 10$ equiv) and sodium bicarbonate (2321 mg, $27.63 \mathrm{mmol}, 15$ equiv) in $25 \mathrm{~mL}$ of water. After stirring at room temperature for 3 hours, the mixture was then dissolved in $50 \mathrm{~mL}$ of water and extracted with dichloromethane $(3 \times 45 \mathrm{~mL})$. The organic layer was dried over $\mathrm{Na}_{2} \mathrm{SO}_{4}$ and filtered, and the solvent was evaporated under reduced pressure. The residue was triturated in ethyl acetate to afford a brown powder of 22a $(567 \mathrm{mg}, 61 \%)$ : $\mathrm{mp} 208{ }^{\circ} \mathrm{C}$; IR $\left(\mathrm{CHCl}_{3}\right) \mathrm{U}_{\max }\left(\mathrm{cm}^{-1}\right): 3284$, 2936, 2791, 1716, 1661, 1636, 1549, 1460, 1370, 1095; ${ }^{1} \mathrm{H}$ NMR $\left(300 \mathrm{MHz}, \mathrm{CDCl}_{3}\right) \delta 0.83$ $(3 \mathrm{H}, \mathrm{s}, \mathrm{H}-18), 0.87$ (3H, d, $J=6.4 \mathrm{~Hz}, \mathrm{H}-21), 0.92(3 \mathrm{H}, \mathrm{s}, \mathrm{H}-30), 1.06,1.07$ (6H, 2d, $J=7.0$ Hz, H-3', H-4'), 1.15 (1H, m, H-6ß), $1.21(1 \mathrm{H}, \mathrm{d}, J=4.0 \mathrm{~Hz}, \mathrm{H}-19 \beta), 1.23$ (3H, s, H-28), $1.35(1 \mathrm{H}, \mathrm{m}, \mathrm{H}-1 \alpha), 1.40(1 \mathrm{H}, \mathrm{m}, \mathrm{H}-7 \alpha), 1.46(1 \mathrm{H}, \mathrm{m}, \mathrm{H}-7 \beta), 1.49(1 \mathrm{H}, \mathrm{bd}, J=14.3 \mathrm{~Hz}, \mathrm{H}-$

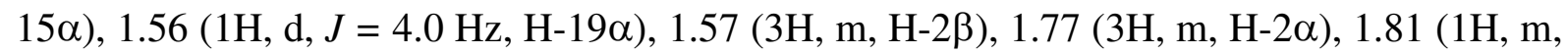

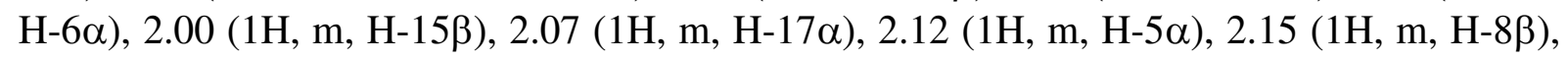
$2.24\left(6 \mathrm{H}, \mathrm{bs}, \mathrm{NB}-\mathrm{CH}_{3}\right), 2.26(1 \mathrm{H}, \mathrm{dq}, J=7.0 \mathrm{~Hz}, \mathrm{H}-2$ '), $2.31(1 \mathrm{H}, \mathrm{d}, J=17.1 \mathrm{~Hz}, \mathrm{H}-12 \beta)$, $2.42(1 \mathrm{H}, \mathrm{m}, \mathrm{H}-1 \beta), 2.52(1 \mathrm{H}, \mathrm{d}, J=17.3 \mathrm{~Hz}, \mathrm{H}-12 \alpha), 2.62(1 \mathrm{H}, \mathrm{dq}, J=6.4,10.9 \mathrm{~Hz}, \mathrm{H}-20)$, 4.09 (1H, ddd, $J=2.1,7.3,9.7 \mathrm{~Hz}, \mathrm{H}-16 \beta), 4.31(1 \mathrm{H}, \mathrm{ddd}, J=4.2,8.9,12.4 \mathrm{~Hz}, \mathrm{H}-3 \alpha), 5.29$ $(1 \mathrm{H}, \mathrm{d}, J=9.1 \mathrm{~Hz}, \mathrm{NAH}), 9.40(1 \mathrm{H}, \mathrm{s}, \mathrm{H}-29) ;{ }^{13} \mathrm{C} \mathrm{NMR}\left(300 \mathrm{MHz}, \mathrm{CDCl}_{3}\right) \delta 7.8\left(\mathrm{CH}_{3}, \mathrm{C}-\right.$ 30), $10.1\left(\mathrm{CH}_{3}, \mathrm{C}-21\right), 18.0\left(\mathrm{CH}_{3}, \mathrm{C}-18\right), 19.4,19.9\left(\mathrm{CH}_{3}, \mathrm{C}-3\right.$ ', C-4'), $20.8\left(\mathrm{CH}_{3}, \mathrm{C}-28\right), 21.2$ $\left(\mathrm{CH}_{2}, \mathrm{C}-6\right), 24.1\left(\mathrm{CH}_{2}, \mathrm{C}-7\right), 27.5\left(\mathrm{CH}_{2}, \mathrm{C}-2\right), 27.6\left(\mathrm{CH}_{2}, \mathrm{C}-1\right), 29.6\left(\mathrm{CH}_{2}, \mathrm{C}-19\right), 34.1(\mathrm{C}, \mathrm{C}-$ 9), 35.7 (CH, C-2'), 35.9 (C, C-10), 41.5 (CH, C-8), 41.9 (CH, C-5), $42.8\left(\mathrm{CH}_{2}, \mathrm{C}-15\right), 44.6$ (C, C-13), $47.2(\mathrm{C}, \mathrm{C}-14), 49.7(\mathrm{CH}, \mathrm{C}-3), 51.5\left(\mathrm{CH}_{2}, \mathrm{C}-12\right), 56.0(\mathrm{CH}, \mathrm{C}-17), 57.0(\mathrm{C}, \mathrm{C}-4)$, 62.2 (CH, C-20), 78.3 (CH, C-16), 176.4 (C, C-1'), 203.4 (C, C-29), 210.9 (C, C-11); ES-MS $m / z 501.4[\mathrm{M}+\mathrm{H}]^{+}$(100), 502.4 (15); HRES-MS $\mathrm{m} / z .501 .3678$ (calcd for $\mathrm{C}_{30} \mathrm{H}_{49} \mathrm{~N}_{2} \mathrm{O}_{4}$, 501.3692). 


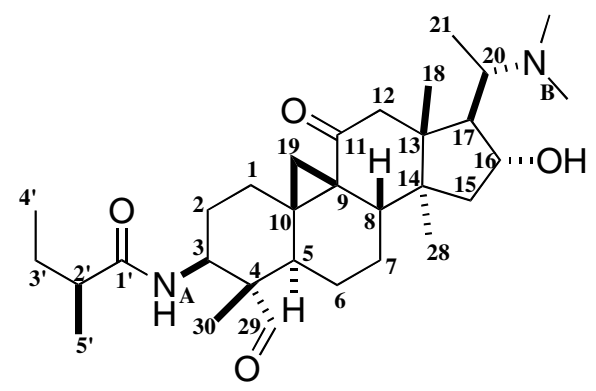

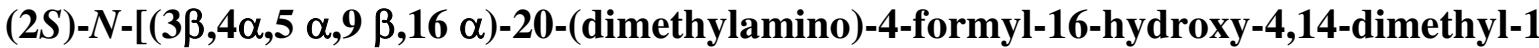 1-oxo-9,19-cyclopregnan-3-yl]-2-methylbutanamide \\ $(22 b)$}

To a stirred solution of compound $\mathbf{2 1 b}(1127 \mathrm{mg}, 2.02 \mathrm{mmol}, 1$ equiv) in $26 \mathrm{~mL}$ of methanol was added a suspension of sodium carbonate $(2138 \mathrm{mg}, 20.20 \mathrm{mmol}, 10$ equiv) and sodium bicarbonate ( $2542 \mathrm{mg}, 30.30 \mathrm{mmol}, 15$ equiv) in $28 \mathrm{~mL}$ of water. After stirring at $60{ }^{\circ} \mathrm{C}$ for 6 hours, the mixture was then dissolved in $50 \mathrm{~mL}$ of water and extracted with dichloromethane (3x50 mL). The organic layer was dried over $\mathrm{Na}_{2} \mathrm{SO}_{4}$ and filtered, and the solvent was evaporated under reduced pressure. The residue was purified by column chromatography on alumina with dichloromethane-methanol (98:2) as eluent followed by a trituration in acetone to afford a colorless powder of $\mathbf{2 1 b}\left(710 \mathrm{mg}, 68 \%\right.$ in 2 steps): $\mathrm{mp} 242{ }^{\circ} \mathrm{C}$; IR $\left(\mathrm{CHCl}_{3}\right)$ $\mathrm{U}_{\max }\left(\mathrm{cm}^{-1}\right): 3287,2935,2709,1717,1659,1640,1548,1454,1372,1229,1093 ;{ }^{1} \mathrm{H}$ NMR $\left(300 \mathrm{MHz}, \mathrm{CDCl}_{3}\right) \delta 0.74(3 \mathrm{H}, \mathrm{s}, \mathrm{H}-18), 0.77(3 \mathrm{H}, \mathrm{t}, J=7.5 \mathrm{~Hz}, \mathrm{H}-4$ '), $0.81(3 \mathrm{H}, \mathrm{d}, J=6.4$ Hz, H-21), 0.87 (3H, s, H-30), 0.98 (1H, m, H-6ß), 1.00 (3H, d, J = 6.8 Hz, H-5'), 1.01 (1H,

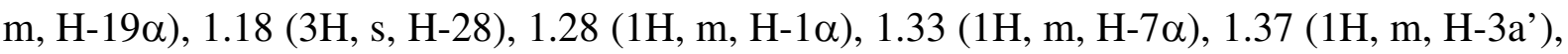
1.39 (1H, m, H-7ß), 1.45 (1H, m, H-3b'), $1.46(1 \mathrm{H}, \mathrm{dd}, J=2.5,14.0 \mathrm{~Hz}, \mathrm{H}-15 \alpha), 1.50$ (1H, d,

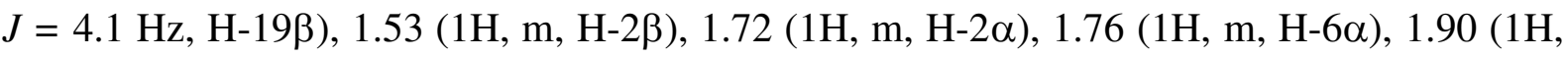

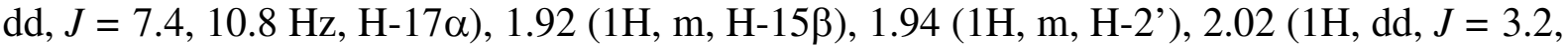

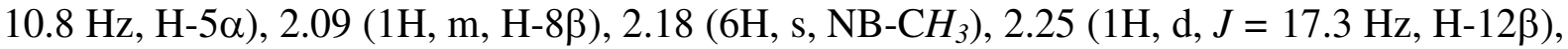
$2.36(1 \mathrm{H}, \mathrm{ddd}, J=3.0,4.7,12.6 \mathrm{~Hz}, \mathrm{H}-1 \beta), 2.47(1 \mathrm{H}, \mathrm{d}, J=17.3 \mathrm{~Hz}, \mathrm{H}-12 \alpha), 2.55$ (1H, dd, $J$ $=6.8,10.8 \mathrm{~Hz}, \mathrm{H}-20), 4.03(1 \mathrm{H}$, ddd, $J=2.8,7.4,10.0 \mathrm{~Hz}, \mathrm{H}-16 \beta), 4.28(1 \mathrm{H}, \mathrm{ddd}, J=4.2$, 8.7, $12.4 \mathrm{~Hz}, \mathrm{H}-3 \alpha), 5.28(1 \mathrm{H}, \mathrm{d}, J=9.1 \mathrm{~Hz}, \mathrm{NA} H), 9.36(1 \mathrm{H}, \mathrm{s}, \mathrm{H}-29) ;{ }^{13} \mathrm{C}$ NMR $(300 \mathrm{MHz}$, $\left.\mathrm{CDCl}_{3}\right) \delta 7.7\left(\mathrm{CH}_{3}, \mathrm{C}-30\right), 10.0\left(\mathrm{CH}_{3}, \mathrm{C}-21\right), 11.7\left(\mathrm{CH}_{3}, \mathrm{C}-4\right), 17.3\left(\mathrm{CH}_{3}, \mathrm{C}-5\right), 17.9\left(\mathrm{CH}_{3}\right.$, C-18), $20.7\left(\mathrm{CH}_{3}, \mathrm{C}-28\right), 21.0\left(\mathrm{CH}_{2}, \mathrm{C}-6\right), 24.0\left(\mathrm{CH}_{2}, \mathrm{C}-7\right), 27.3\left(\mathrm{CH}_{2}, \mathrm{C}-1\right), 27.5\left(\mathrm{CH}_{2}, \mathrm{C}-\right.$ 3'), $27.5\left(\mathrm{CH}_{2}, \mathrm{C}-2\right), 29.6\left(\mathrm{CH}_{2}, \mathrm{C}-19\right), 34.0(\mathrm{C}, \mathrm{C}-9), 35.8$ (C, C-10), $41.3(\mathrm{CH}, \mathrm{C}-5), 41.7$ $(\mathrm{CH}, \mathrm{C}-8), 41.8\left(\mathrm{CH}_{3}, \mathrm{NB}-\mathrm{CH}_{3}\right), 42.6\left(\mathrm{CH}_{2}, \mathrm{C}-15\right), 43.2(\mathrm{CH}, \mathrm{C}-2$ ') $, 44.4(\mathrm{C}, \mathrm{C}-13), 47.1(\mathrm{C}$, C-14), 49.8 (CH, C-3), $51.4\left(\mathrm{CH}_{2}, \mathrm{C}-12\right), 55.8$ (CH, C-17), 56.8 (C, C-4), 62.0 (CH, C-20), 78.2 (CH, C-16), 175.8 (C, C-1'), 203.4 (C, C-29), 210.9 (C, C-11); ES-MS m/z 515.4 $[\mathrm{M}+\mathrm{H}]^{+}$(100), 516.4 (5); HRES-MS $m / z, 515.3831$ (calcd for $\mathrm{C}_{31} \mathrm{H}_{51} \mathrm{~N}_{2} \mathrm{O}_{4}, 515.3849$ ). 


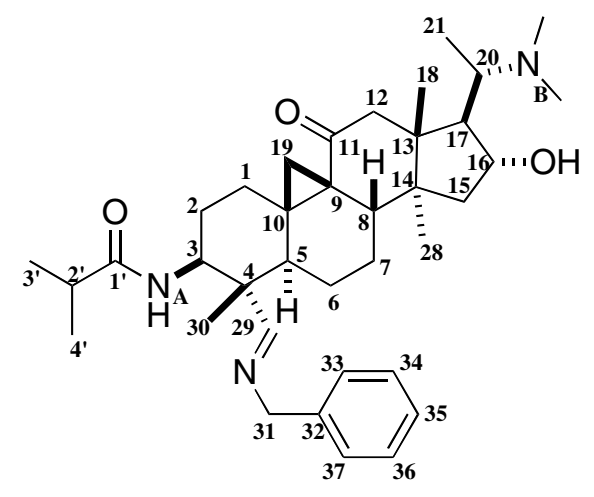

\section{Compound (23a)}

To a stirred suspension of compound 22a $(890 \mathrm{mg}, 1.78 \mathrm{mmol}, 1.0$ equiv) and $900 \mathrm{mg}$ of anhydrous magnesium sulfate in $10 \mathrm{~mL}$ of freshly distilled dichloromethane was added benzylamine $\left(253 \mu \mathrm{L}, 2.31 \mathrm{mmol}, 1.3\right.$ equiv). After stirring at $55{ }^{\circ} \mathrm{C}$ for 17 hours, the mixture was then filtered on celite, and the solvent was evaporated under reduced pressure to afford a colorless powder of 23a $(1.05 \mathrm{~g}, 99 \%)$ : IR $\left(\mathrm{CHCl}_{3}\right) \mathrm{v}_{\max }\left(\mathrm{cm}^{-1}\right): 3386,2958-2928,1678,1649$, 1529, 1451, 1378, 1227, 1091; ${ }^{1} \mathrm{H}$ NMR $\left(300 \mathrm{MHz}, \mathrm{CDCl}_{3}\right) \delta 0.77(3 \mathrm{H}, \mathrm{s}, \mathrm{H}-18), 0.81(3 \mathrm{H}, \mathrm{d}$, $J=6.6 \mathrm{~Hz}, \mathrm{H}-21), 0.93$ (1H, m, H-6ß), 0.93, 0.96 (6H, 2d, $J=6.8 \mathrm{~Hz}, \mathrm{H}-3$ ', H-4'), 0.97 (3H, s, H-30), $1.02(1 \mathrm{H}, \mathrm{d}, J=4.0 \mathrm{~Hz}, \mathrm{H}-19 \alpha), 1.16(3 \mathrm{H}, \mathrm{s}, \mathrm{H}-28), 1.26(1 \mathrm{H}, \mathrm{m}, \mathrm{H}-1 \alpha), 1.30(1 \mathrm{H}$, m, H-7 $\alpha), 1.34(1 \mathrm{H}, \mathrm{m}, \mathrm{H}-7 \beta), 1.40(1 \mathrm{H}, \mathrm{m}, \mathrm{H}-2 \beta), 1.42(1 \mathrm{H}, \mathrm{dd}, J=3.0,13.9 \mathrm{~Hz}, \mathrm{H}-15 \alpha)$, $1.50(1 \mathrm{H}, \mathrm{m}, \mathrm{H}-6 \alpha), 1.53(1 \mathrm{H}, \mathrm{d}, J=4.0 \mathrm{~Hz}, \mathrm{H}-19 \beta), 1.76(1 \mathrm{H}, \mathrm{m}, \mathrm{H}-2 \alpha), 1.87(1 \mathrm{H}, \mathrm{m}, \mathrm{H}-$ $5 \alpha), 1.93(1 \mathrm{H}, \mathrm{m}, \mathrm{H}-17 \alpha), 1.99(1 \mathrm{H}, \mathrm{m}, \mathrm{H}-15 \beta), 2.07(1 \mathrm{H}, \mathrm{m}, \mathrm{H}-8 \beta), 2.10(1 \mathrm{H}, \mathrm{dq}, J=6.8$ Hz, H-2'), 2.19 (6H, s, NB-CH $\left.{ }_{3}\right), 2.25(1 \mathrm{H}, \mathrm{d}, J=17.3 \mathrm{~Hz}, \mathrm{H}-12 \beta), 2.31$ (1H, ddd, $J=3.3$, 3.6, $13.4 \mathrm{~Hz}, \mathrm{H}-1 \beta), 2.45(1 \mathrm{H}, \mathrm{d}, J=17.3 \mathrm{~Hz}, \mathrm{H}-12 \alpha), 2.55(1 \mathrm{H}, \mathrm{dd}, J=6.8,10.8 \mathrm{~Hz}, \mathrm{H}-20)$, $4.03(2 \mathrm{H}, \mathrm{m}, \mathrm{H}-3 \alpha, \mathrm{H}-16 \beta), 4.45,4.49(2 \mathrm{H}, 2 \mathrm{~d}, J=15.2 \mathrm{~Hz}, \mathrm{H}-31), 5.26(1 \mathrm{H}, \mathrm{d}, J=9.2 \mathrm{~Hz}$, NAH $), 7.14-7.27(5 \mathrm{H}, \mathrm{m}, \mathrm{HAr}=33,34,35,36,37), 7.49(1 \mathrm{H}, \mathrm{s}, \mathrm{H}-29) ;{ }^{13} \mathrm{C}$ NMR $(300 \mathrm{MHz}$, $\left.\mathrm{CDCl}_{3}\right) \delta 10.0\left(\mathrm{CH}_{3}, \mathrm{C}-21\right), 10.2\left(\mathrm{CH}_{3}, \mathrm{C}-30\right), 17.9\left(\mathrm{CH}_{2}, \mathrm{C}-6\right), 17.9\left(\mathrm{CH}_{3}, \mathrm{C}-18\right), 19.3,19.6$ $\left(\mathrm{CH}_{3}, \mathrm{C}-3\right.$ ', C-4'), $20.7\left(\mathrm{CH}_{3}, \mathrm{C}-28\right), 24.2\left(\mathrm{CH}_{2}, \mathrm{C}-7\right), 27.6\left(\mathrm{CH}_{2}, \mathrm{C}-2\right), 27.7\left(\mathrm{CH}_{2}, \mathrm{C}-1\right), 29.8$ $\left(\mathrm{CH}_{2}, \mathrm{C}-19\right), 34.3$ (C, C-9), 35.7 (CH, C-2'), 36.7 (C, C-10), $41.3(\mathrm{CH}, \mathrm{C}-8), 42.7\left(\mathrm{CH}_{2}, \mathrm{C}-\right.$ 15), 44.5 (C, C-13), 44.8 (CH, C-5), 47.2 (C, C-14), 50.0 (C, C-4), $51.4\left(\mathrm{CH}_{2}, \mathrm{C}-12\right), 52.0$ (CH, C-3), 55.9 (CH, C-17), $62.1(\mathrm{CH}, \mathrm{C}-20), 64.7\left(\mathrm{CH}_{2}, \mathrm{C}-31\right), 78.2(\mathrm{CH}, \mathrm{C}-16), 126.7(\mathrm{CH}$, C-35), 127.7, 128.3 (CH, C-33, C-34, C36, C-37), 139.5 (C, C-32), 170.7 (CH, C-29), 176.0 (C, C-1'), 210.9 (C, C-11); ES-MS m/z 590.4 [M+H] ${ }^{+}$(100), 591.4 (10); HRES-MS m/z 590.4304 (calcd for $\mathrm{C}_{37} \mathrm{H}_{55} \mathrm{~N}_{3} \mathrm{O}_{3}, 590.4322$ ). 


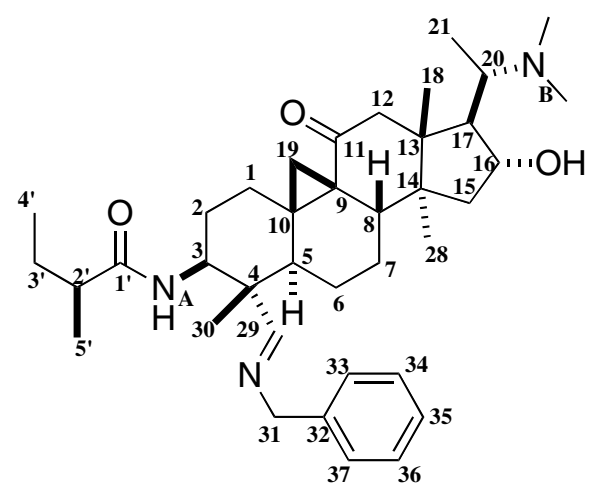

\section{Compound (23b)}

To a stirred suspension of compound $22 \mathbf{b}(650 \mathrm{mg}, 1.26 \mathrm{mmol}, 1.0$ equiv) and $540 \mathrm{mg}$ of anhydrous magnesium sulfate in $5 \mathrm{~mL}$ of freshly distilled dichloromethane was added benzylamine $\left(179 \mu \mathrm{L}, 1.63 \mathrm{mmol}, 1.3\right.$ equiv). After stirring at $55^{\circ} \mathrm{C}$ for 10 hours, the mixture was then filtered on celite, and the solvent was evaporated under reduced pressure to afford a colorless powder of 23b (793 mg, 99\%): IR $\left(\mathrm{CHCl}_{3}\right) v_{\max }\left(\mathrm{cm}^{-1}\right): 3386,2958-2927,1649$, $1529,1451,1364,1227,1091 ;{ }^{1} \mathrm{H}$ NMR $\left(300 \mathrm{MHz}, \mathrm{CDCl}_{3}\right) \delta 0.73(3 \mathrm{H}, \mathrm{t}, J=7.5 \mathrm{~Hz}, \mathrm{H}-4$ '), $0.77(3 \mathrm{H}, \mathrm{s}, \mathrm{H}-18), 0.81(3 \mathrm{H}, \mathrm{d}, J=6.6 \mathrm{~Hz}, \mathrm{H}-21), 0.93(1 \mathrm{H}, \mathrm{m}, \mathrm{H}-6 \beta), 0.95(3 \mathrm{H}, \mathrm{d}, J=6.8$

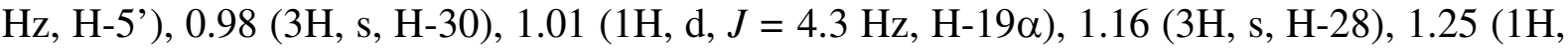

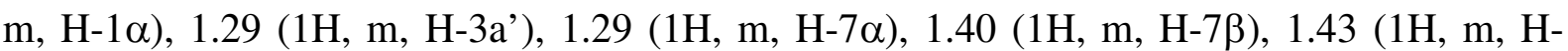

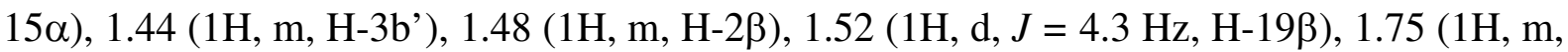

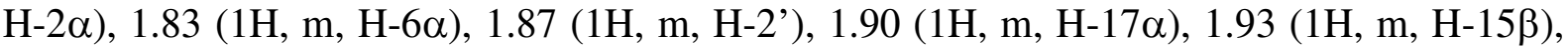
$1.98(1 \mathrm{H}, \mathrm{m}, \mathrm{H}-5 \alpha), 2.00(1 \mathrm{H}, \mathrm{m}, \mathrm{H}-8 \beta), 2.19\left(6 \mathrm{H}, \mathrm{s}, \mathrm{NB}-\mathrm{CH}_{3}\right), 2.24(1 \mathrm{H}, \mathrm{d}, J=17.3 \mathrm{~Hz}, \mathrm{H}-$ $12 \beta), 2.31(1 \mathrm{H}$, ddd, $J=3.2,3.3,13.2 \mathrm{~Hz}, \mathrm{H}-1 \beta), 2.46(1 \mathrm{H}, \mathrm{d}, J=17.3 \mathrm{~Hz}, \mathrm{H}-12 \alpha), 2.56$ $(1 \mathrm{H}, \mathrm{dd}, J=6.6,12.0 \mathrm{~Hz}, \mathrm{H}-20), 4.03$ (2H, m, H-3, $\mathrm{H}-16 \beta), 4.43,4.50(2 \mathrm{H}, 2 \mathrm{~d}, J=14.1 \mathrm{~Hz}$, H-31), $5.25(1 \mathrm{H}, \mathrm{d}, J=9.2 \mathrm{~Hz}, \mathrm{NAH}), 7.14-7.25(5 \mathrm{H}, \mathrm{m}, \mathrm{HAr}=33,24,35,36,37), 7.50(1 \mathrm{H}$, s, H-29); ${ }^{13} \mathrm{C}$ NMR $\left(300 \mathrm{MHz}, \mathrm{CDCl}_{3}\right) \delta 10.0\left(\mathrm{CH}_{3}, \mathrm{C}-30\right), 10.3\left(\mathrm{CH}_{3}, \mathrm{C}-21\right), 11.8\left(\mathrm{CH}_{3}, \mathrm{C}-\right.$ 4'), $17.1\left(\mathrm{CH}_{3}, \mathrm{C}-5^{\prime}\right), 17.9\left(\mathrm{CH}_{3}, \mathrm{C}-18\right), 20.6\left(\mathrm{CH}_{2}, \mathrm{C}-6\right), 20.7\left(\mathrm{CH}_{3}, \mathrm{C}-28\right), 24.2\left(\mathrm{CH}_{2}, \mathrm{C}-7\right)$, $27.2\left(\mathrm{CH}_{2}, \mathrm{C}-3\right.$ ') $27.5\left(\mathrm{CH}_{2}, \mathrm{C}-1\right), 27.7\left(\mathrm{CH}_{2}, \mathrm{C}-2\right), 29.8\left(\mathrm{CH}_{2}, \mathrm{C}-19\right), 34.3(\mathrm{C}, \mathrm{C}-9), 36.7(\mathrm{C}$, C-10), 41.3 (CH, C-8), $42.7\left(\mathrm{CH}_{2}, \mathrm{C}-15\right), 43.3$ (CH, C-2'), 44.5 (C, C-13), 44.7 (CH, C-5), 47.1 (C, C-14), 49.9 (C, C-4), $51.4\left(\mathrm{CH}_{2}, \mathrm{C}-12\right), 52.2(\mathrm{CH}, \mathrm{C}-3), 55.8(\mathrm{CH}, \mathrm{C}-17), 62.1(\mathrm{CH}$, C-20), $64.7\left(\mathrm{CH}_{2}, \mathrm{C}-31\right), 78.2(\mathrm{CH}, \mathrm{C}-16), 126.7(\mathrm{CH}, \mathrm{C}-35), 127.7,128.3(\mathrm{CH}, \mathrm{C}-33, \mathrm{C}-34$, C-36, C-37), 139.5 (C, C-32), 170.9 (C, C-29), 175.6 (C, C-1'), 211.0 (C, C-11); ES-MS m/z $604.4[\mathrm{M}+\mathrm{H}]^{+}(100), 605.4$ (55), 590.4 (30); HRES-MS m/z 604.4468 (calcd for $\mathrm{C}_{38} \mathrm{H}_{58} \mathrm{~N}_{3} \mathrm{O}_{3}$, 604.4478). 


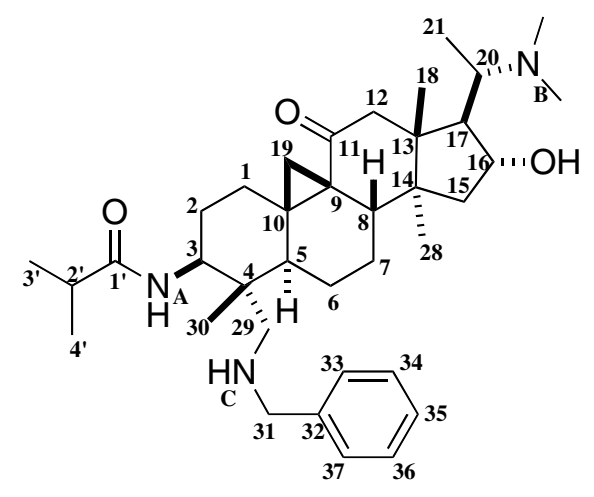

\section{Compound (24a)}

To a stirred solution of crude compound 23a (2.98 g, $5.05 \mathrm{mmol}, 1.00$ equiv) in $30 \mathrm{~mL}$ of methanol was added sodium cyanoborohydride (381 mg, $6.06 \mathrm{mmol}, 1.20$ equiv) and glacial acetic acid $(289 \mu \mathrm{L}, 5.06 \mathrm{mmol}, 1.05$ equiv). After stirring at room temperature for 3 hours, the mixture was then made basic with $50 \mathrm{~mL}$ of a $10 \%$ ammonia solution and extracted with dichloromethane $(3 \times 50 \mathrm{~mL})$. The organic layer was dried over $\mathrm{Na}_{2} \mathrm{SO}_{4}$ and filtered, and the solvent was evaporated under reduced pressure. The residue was purified by column chromatography on silica with dichloromethane-methanol-ammonia $(97.5: 0.5: 2)$ mixture as eluent to afford a yellow powder of $\mathbf{2 4 a}\left(2.64 \mathrm{~g}, 88 \%\right.$ for 2 steps): $\mathrm{mp} 211{ }^{\circ} \mathrm{C}$; $[\alpha]^{23}{ }_{\mathrm{D}}=+96$ (c $\left.0.45, \mathrm{CHCl}_{3}\right)$; IR $\left(\mathrm{CHCl}_{3}\right) \cup_{\max }\left(\mathrm{cm}^{-1}\right): 3312,2933,1660,1534,1452,1378,1226,1095 ;{ }^{1} \mathrm{H}$ NMR $\left(300 \mathrm{MHz}, \mathrm{CDCl}_{3}\right) \delta 0.51(3 \mathrm{H}, \mathrm{s}, \mathrm{H}-30), 0.65(1 \mathrm{H}, \mathrm{m}, \mathrm{H}-6 \beta), 0.76(3 \mathrm{H}, \mathrm{s}, \mathrm{H}-18), 0.80$ $(3 \mathrm{H}, \mathrm{d}, J=6.6 \mathrm{~Hz}, \mathrm{H}-21), 1.07(6 \mathrm{H}, 2 \mathrm{~d}, J=7.0 \mathrm{~Hz}, \mathrm{H}-3$ ', H-4'), $0.92(1 \mathrm{H}, \mathrm{d}, J=3.4 \mathrm{~Hz}, \mathrm{H}-$

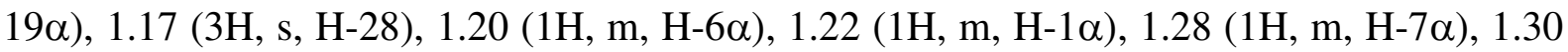
$(1 \mathrm{H}, \mathrm{m}, \mathrm{H}-15 \alpha), 1.33(1 \mathrm{H}, \mathrm{m}, \mathrm{H}-7 \beta), 1.37(1 \mathrm{H}, \mathrm{m}, \mathrm{H}-2 \beta), 1.45(1 \mathrm{H}, \mathrm{d}, J=3.4 \mathrm{~Hz}, \mathrm{H}-19 \beta)$,

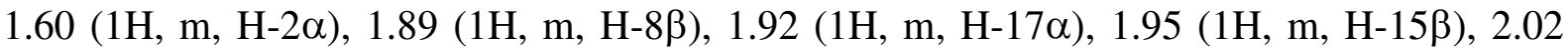
$(1 \mathrm{H}, \mathrm{d}, J=13.0 \mathrm{~Hz}, \mathrm{H}-29 \mathrm{a}), 2.12(1 \mathrm{H}, \mathrm{m}, \mathrm{H}-5 \alpha), 2.19\left(6 \mathrm{H}, \mathrm{s}, \mathrm{NB}-\mathrm{CH}_{3}\right), 2.14(1 \mathrm{H}, \mathrm{d}, J=13.0$ Hz, H-29b), 2.22 (1H, d, $J=17.1 \mathrm{~Hz}, \mathrm{H}-12 \beta), 2.24$ (1H, dq, $J=7.0 \mathrm{~Hz}, \mathrm{H}-2$ '), $2.32(1 \mathrm{H}, \mathrm{m}$,

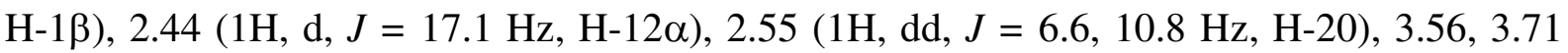
$(2 \mathrm{H}, 2 \mathrm{~d}, J=13.8 \mathrm{~Hz}, \mathrm{H}-31), 4.00(1 \mathrm{H}, \mathrm{m}, \mathrm{H}-3 \alpha), 4.03(1 \mathrm{H}, \mathrm{m}, \mathrm{H}-16 \beta), 5.10(1 \mathrm{H}, \mathrm{d}, J=10.0$ $\mathrm{Hz}, \mathrm{NAH}), 7.19-7.28(5 \mathrm{H}, \mathrm{m}, \mathrm{HAr}=33,34,35,36,37) ;{ }^{13} \mathrm{C} \mathrm{NMR}\left(300 \mathrm{MHz}, \mathrm{CDCl}_{3}\right) \delta 9.9$ $\left(\mathrm{CH}_{3}, \mathrm{C}-21\right), 13.7\left(\mathrm{CH}_{3}, \mathrm{C}-30\right), 17.8\left(\mathrm{CH}_{3}, \mathrm{C}-18\right), 18.5\left(\mathrm{CH}_{2}, \mathrm{C}-6\right), 19.5,20.2\left(\mathrm{CH}_{3}, \mathrm{C}-3\right), \mathrm{C}-$ 4'), $21.0\left(\mathrm{CH}_{3}, \mathrm{C}-28\right), 24.5\left(\mathrm{CH}_{2}, \mathrm{C}-7\right), 27.6\left(\mathrm{CH}_{2}, \mathrm{C}-2\right), 28.4\left(\mathrm{CH}_{2}, \mathrm{C}-1\right), 31.0\left(\mathrm{CH}_{2}, \mathrm{C}-19\right)$, 34.6 (C, C-9), 36.0 (CH, C-2'), 38.2 (C, C-10), 41.7 (CH, C-8), 42.1 (CH, C-5), $42.9\left(\mathrm{CH}_{2}, \mathrm{C}-\right.$ 15), 43.2 (C, C-13), 44.5 (C, C-4), 47.2 (C, C-14), $50.3(\mathrm{CH}, \mathrm{C}-3), 51.5\left(\mathrm{CH}_{2}, \mathrm{C}-12\right), 52.9$ $\left(\mathrm{CH}_{2}, \mathrm{C}-29\right), 54.5\left(\mathrm{CH}_{2}, \mathrm{C}-31\right), 55.8(\mathrm{CH}, \mathrm{C}-17), 62.1(\mathrm{CH}, \mathrm{C}-20), 78.4(\mathrm{CH}, \mathrm{C}-16), 126.5$ (CH, C-35), 128.0, 128.4 (CH, C-33, C-34, C36, C-37), 141.2 (C, C-32), 176.6 (C, C-1'), 211.6 (C, C-11); ES-MS m/z $592.5[\mathrm{M}+\mathrm{H}]^{+}$(100), 593.5 (10), 296.7 (5); HRES-MS $\mathrm{m} / \mathrm{z}$ 592.4451 (calcd for $\mathrm{C}_{37} \mathrm{H}_{58} \mathrm{~N}_{3} \mathrm{O}_{3}, 592.4478$ ). 


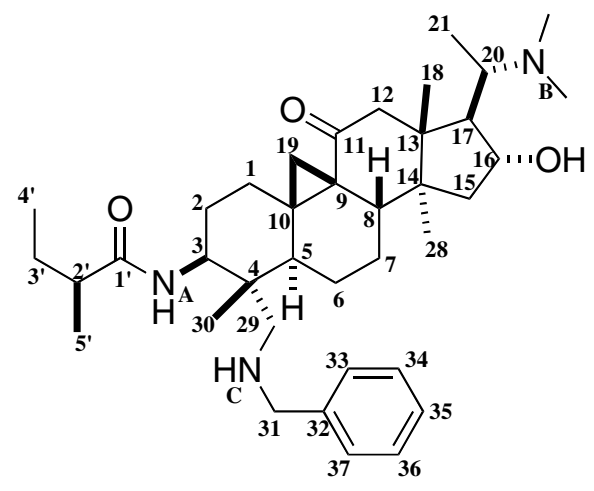

\section{Compound (24b)}

To a stirred solution of crude compound $\mathbf{2 3 b}$ (770 mg, $1.26 \mathrm{mmol}, 1.00$ equiv) in $7 \mathrm{~mL}$ of methanol was added sodium cyanoborohydride (102 mg, $1.54 \mathrm{mmol}, 1.20$ equiv) and glacial acetic acid ( $39 \mu \mathrm{L}, 1.27 \mathrm{mmol}, 1.05$ equiv). After stirring at room temperature for 3 hours, the mixture was then made basic with $50 \mathrm{~mL}$ of a $10 \%$ ammonia solution and extracted with dichloromethane $(3 \times 50 \mathrm{~mL})$. The organic layer was dried over $\mathrm{Na}_{2} \mathrm{SO}_{4}$ and filtered, and the solvent was evaporated under reduced pressure. The residue was purified by column chromatography on alumina with dichloromethane-methanol (98:2) as eluent to afford a colorless powder of $\mathbf{2 4 b}\left(618 \mathrm{mg}, 81 \%\right.$ in 2 steps): IR $\left(\mathrm{CHCl}_{3}\right) \mathrm{v}_{\max }\left(\mathrm{cm}^{-1}\right): 3314,2932,1660$, $1529,1452,1377,1226,1094 ;{ }^{1} \mathrm{H}$ NMR $\left(300 \mathrm{MHz}, \mathrm{CDCl}_{3}\right) \delta 0.59(3 \mathrm{H}, \mathrm{s}, \mathrm{H}-18), 0.83(3 \mathrm{H}, \mathrm{s}$,

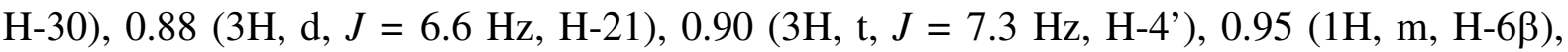
$1.09(1 \mathrm{H}, \mathrm{d}, J=3.4 \mathrm{~Hz}, \mathrm{H}-19 \alpha), 1.13(3 \mathrm{H}, \mathrm{d}, J=6.8 \mathrm{~Hz}, \mathrm{H}-5$ '), $1.25(3 \mathrm{H}, \mathrm{s}, \mathrm{H}-28), 1.33(1 \mathrm{H}$,

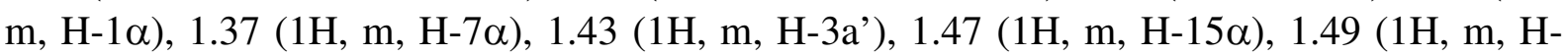
$7 \beta), 1.50(1 \mathrm{H}, \mathrm{m}, \mathrm{H}-2 \beta), 1.52(1 \mathrm{H}, \mathrm{d}, J=3.4 \mathrm{~Hz}, \mathrm{H}-19 \beta), 1.65(1 \mathrm{H}, \mathrm{m}, \mathrm{H}-2 \alpha), 1.66(1 \mathrm{H}, \mathrm{m}$,

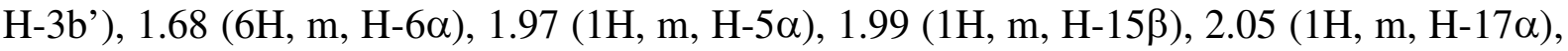
$2.09(1 \mathrm{H}, \mathrm{m}, \mathrm{H}-8 \beta), 2.12(1 \mathrm{H}, \mathrm{d}, J=12.2 \mathrm{~Hz}, \mathrm{H}-29 \mathrm{a}), 2.25(1 \mathrm{H}, \mathrm{m}, \mathrm{H}-2$ ') 2.25 (6H, s, NB$\left.\mathrm{CH}_{3}\right), 2.27(1 \mathrm{H}, \mathrm{d}, J=12.2 \mathrm{~Hz}, \mathrm{H}-29 \mathrm{~b}), 2.29(1 \mathrm{H}, \mathrm{d}, J=17.1 \mathrm{~Hz}, \mathrm{H}-12 \beta), 2.36(1 \mathrm{H}, \mathrm{m}, \mathrm{H}-$ $1 \beta), 2.52(1 \mathrm{H}, \mathrm{d}, J=17.1 \mathrm{~Hz}, \mathrm{H}-12 \alpha), 2.62(1 \mathrm{H}, \mathrm{dd}, J=6.6,10.8 \mathrm{~Hz}, \mathrm{H}-20), 3.62,3.81(2 \mathrm{H}$, $2 \mathrm{~d}, J=13,7 \mathrm{~Hz}, \mathrm{H}-31), 4.07(1 \mathrm{H}, \mathrm{m}, \mathrm{H}-3 \alpha), 4.11(1 \mathrm{H}, \mathrm{m}, \mathrm{H}-16 \beta), 5.19(1 \mathrm{H}, \mathrm{d}, J=9.8 \mathrm{~Hz}$, NAH $), 7.18-7.35(5 \mathrm{H}, \mathrm{m}, \mathrm{HAr}=33,34,35,36,37) ;{ }^{13} \mathrm{C}$ NMR $\left(300 \mathrm{MHz}, \mathrm{CDCl}_{3}\right) \delta 9.9\left(\mathrm{CH}_{3}\right.$, C-21), $12.1\left(\mathrm{CH}_{3}, \mathrm{C}-4\right.$ ') $\left.13.7\left(\mathrm{CH}_{3}, \mathrm{C}-30\right), 17.7\left(\mathrm{CH}_{3}, \mathrm{C}-5\right)\right), 17.8\left(\mathrm{CH}_{3}, \mathrm{C}-18\right), 18.4\left(\mathrm{CH}_{2}, \mathrm{C}-\right.$ 6), $\left.21.0\left(\mathrm{CH}_{3}, \mathrm{C}-28\right), 24.4\left(\mathrm{CH}_{2}, \mathrm{C}-7\right), 27.5\left(\mathrm{CH}_{2}, \mathrm{C}-3\right)\right), 27.6\left(\mathrm{CH}_{2}, \mathrm{C}-1\right), 28.5\left(\mathrm{CH}_{2}, \mathrm{C}-2\right)$, $31.0\left(\mathrm{CH}_{2}, \mathrm{C}-19\right), 34.6$ (C, C-9), 38.2 (C, C-10), $41.6(\mathrm{CH}, \mathrm{C}-8), 42.0(\mathrm{CH}, \mathrm{C}-5), 42.9\left(\mathrm{CH}_{2}\right.$, C-15), 43.1 (C, C-4), 43.7 (CH, C-2'), 44.5 (C, C-13), 47.1 (C, C-14), 50.5 (CH, C-3), 51.5 $\left(\mathrm{CH}_{2}, \mathrm{C}-12\right), 52.9\left(\mathrm{CH}_{2}, \mathrm{C}-29\right), 54.5\left(\mathrm{CH}_{2}, \mathrm{C}-31\right), 55.8(\mathrm{CH}, \mathrm{C}-17), 62.0(\mathrm{CH}, \mathrm{C}-20), 78.4$ (CH, C-16), 126.5 (CH, C-35), 128.0, 128.4 (CH, C-33, C-34, C-36, C-37), 141.3 (C, C-32), 176.1 (C, C-1'), 211.6 (C, C-11); ES-MS m/z 606.5 [M+H] ${ }^{+}$(100), 607.5 (10); HRES-MS m/z 606.4634 (calcd for $\mathrm{C}_{38} \mathrm{H}_{60} \mathrm{~N}_{3} \mathrm{O}_{3}, 606.4635$ ). 


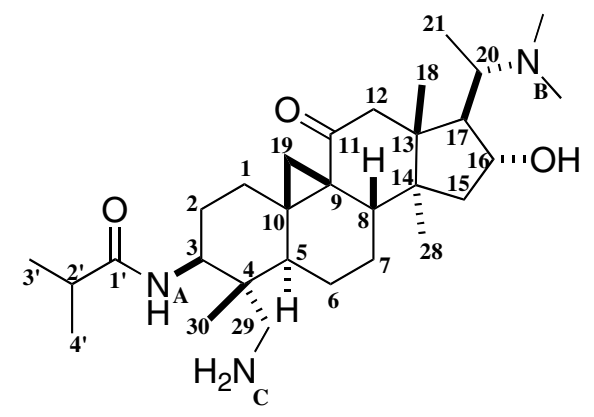

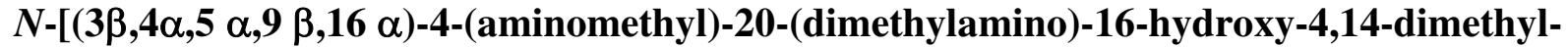 11-oxo-9,19-cyclopregnan-3-yl]-2-methylpropanamide \\ (25a)}

To a stirred suspension of compound 24a (817 mg, $1.38 \mathrm{mmol}, 1$ equiv) in $30 \mathrm{~mL}$ of methanol was added palladium at $30 \%$ on carbon ( $408 \mathrm{mg}, 50 \%$ by weight) and ammonium formate (435 mg, $6.90 \mathrm{mmol}, 5$ equiv). After stirring at $40{ }^{\circ} \mathrm{C}$ for 3 hours, the mixture was then filtered on celite, and the solvent was evaporated under reduced pressure. The residue was then made basic with $40 \mathrm{~mL}$ of a $10 \%$ sodium carbonate solution and extracted with dichloromethane $(3 \times 20 \mathrm{~mL})$. The organic layer was dried over $\mathrm{Na}_{2} \mathrm{SO}_{4}$ and filtered, and the solvent was evaporated under reduced pressure. The residue was purified by column chromatography on silica with dichloromethane-methanol-ammonia (96:2:2) as eluent to afford a colorless powder of 25a $(588 \mathrm{mg}, 85 \%)$ : $\mathrm{mp} 232{ }^{\circ} \mathrm{C}$; IR $\left(\mathrm{CHCl}_{3}\right) \mathrm{U}_{\max }\left(\mathrm{cm}^{-1}\right): 3313$, 2934, 1650, 1536, 1453, 1379, 1226, 1094; ${ }^{1} \mathrm{H}$ NMR (300 MHz, $\left.\mathrm{CDCl}_{3}\right) \delta 0.56(3 \mathrm{H}, \mathrm{s}, \mathrm{H}-30)$, $0.78(3 \mathrm{H}, \mathrm{s}, \mathrm{H}-18), 0.80(3 \mathrm{H}, \mathrm{d}, J=6.6 \mathrm{~Hz}, \mathrm{H}-21), 0.87(1 \mathrm{H}, \mathrm{m}, \mathrm{H}-6 \beta), 0.97(1 \mathrm{H}, \mathrm{d}, J=3.8$ Hz, H-19 $)$ ), 1.08, 1.10 (6H, 2d, $J=6.6$ Hz, H-3', H-4'), 1.15 (3H, s, H-28), 1.23 (1H, m, H-

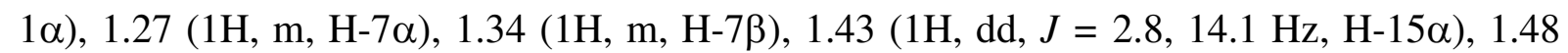

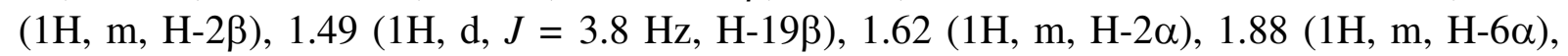

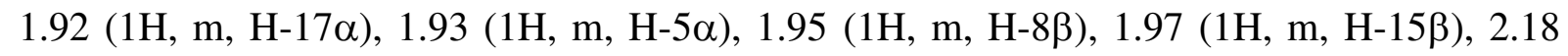
$\left(6 \mathrm{H}, \mathrm{s}, \mathrm{NB}-\mathrm{CH}_{3}\right), 2.24(1 \mathrm{H}, \mathrm{d}, J=17.1 \mathrm{~Hz}, \mathrm{H}-12 \beta), 2.26(1 \mathrm{H}, \mathrm{d}, J=13.9 \mathrm{~Hz}, \mathrm{H}-29 \mathrm{a}), 2.28$ $\left(1 \mathrm{H}, \mathrm{dq}, J=6.6 \mathrm{~Hz}, \mathrm{H}-2^{\prime}\right), 2.38(1 \mathrm{H}, \mathrm{d}, J=13.9 \mathrm{~Hz}, \mathrm{H}-29 \mathrm{~b}), 2.39$ (1H, m, H-1ß), $2.43(1 \mathrm{H}$, $\mathrm{d}, J=17.1 \mathrm{~Hz}, \mathrm{H}-12 \alpha), 2.55(1 \mathrm{H}, \mathrm{dd}, J=6.6,10.9 \mathrm{~Hz}, \mathrm{H}-20), 3.99(1 \mathrm{H}, \mathrm{m}, \mathrm{H}-3 \alpha), 4.03(1 \mathrm{H}$, $\mathrm{m}, \mathrm{H}-16 \beta), 5.13(1 \mathrm{H}, \mathrm{d}, J=9.8 \mathrm{~Hz}, \mathrm{NAH}) ;{ }^{13} \mathrm{C} \mathrm{NMR}\left(300 \mathrm{MHz}, \mathrm{CDCl}_{3}\right) \delta 9.9\left(\mathrm{CH}_{3}, \mathrm{C}-21\right)$, $13.7\left(\mathrm{CH}_{3}, \mathrm{C}-30\right), 17.8\left(\mathrm{CH}_{3}, \mathrm{C}-18\right), 18.5\left(\mathrm{CH}_{2}, \mathrm{C}-6\right), 19.5,20.1\left(\mathrm{CH}_{3}, \mathrm{C}-3\right.$ ', $\left.\mathrm{C}-4{ }^{\prime}\right), 21.0\left(\mathrm{CH}_{3}\right.$, C-28), $24.6\left(\mathrm{CH}_{2}, \mathrm{C}-7\right), 27.7\left(\mathrm{CH}_{2}, \mathrm{C}-2\right), 28.4\left(\mathrm{CH}_{2}, \mathrm{C}-1\right), 31.2\left(\mathrm{CH}_{2}, \mathrm{C}-19\right), 34.4(\mathrm{C}, \mathrm{C}-9)$, 35.9 (CH, C-2'), 38.1 (C, C-10), 41.7 (CH, C-8), 42.0 (CH, C-5), $42.9\left(\mathrm{CH}_{2}, \mathrm{C}-15\right), 43.7$ (C, C-13), 44.5 (C, C-4), $46.2\left(\mathrm{CH}_{2}, \mathrm{C}-29\right), 47.1$ (C, C-14), $49.5(\mathrm{CH}, \mathrm{C}-3), 51.5\left(\mathrm{CH}_{2}, \mathrm{C}-12\right)$, $55.8(\mathrm{CH}, \mathrm{C}-17), 62.0(\mathrm{CH}, \mathrm{C}-20), 78.3(\mathrm{CH}, \mathrm{C}-16), 176.8$ (C, C-1'), 211.5 (C, C-11); ES-MS $\mathrm{m} / z \quad 502.4[\mathrm{M}+\mathrm{H}]^{+}(100), 503.4(10), 296.7(15), 251.7[\mathrm{M}+2 \mathrm{H}]^{+}(10) ; \mathrm{HRES}-\mathrm{MS} \mathrm{m} / \mathrm{z}$ 502.4008 (calcd for $\mathrm{C}_{30} \mathrm{H}_{51} \mathrm{~N}_{3} \mathrm{O}_{3}, 502.4009$ ). 


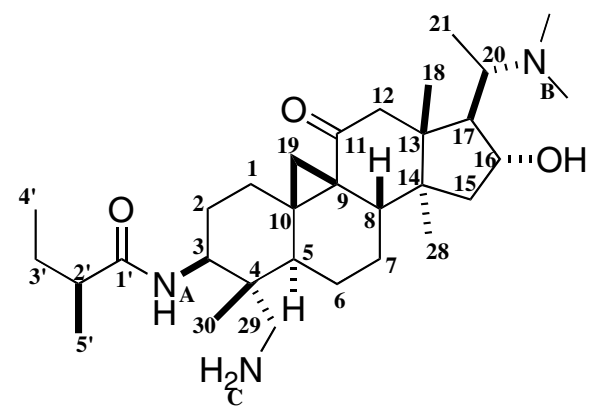

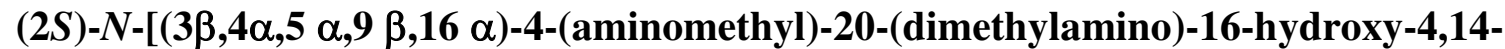
dimethyl-11-oxo-9,19-cyclopregnan-3-yl]-2-methylbutanamide (25b)

To a stirred suspension of compound $24 \mathbf{b}(580 \mathrm{mg}, 0.96 \mathrm{mmol}, 1$ equiv) in $20 \mathrm{~mL}$ of methanol was added palladium at $30 \%$ on carbon $(290 \mathrm{mg}, 50 \%$ by weight) and ammonium formate (311 mg, $4.79 \mathrm{mmol}, 5$ equiv). After stirring at $40{ }^{\circ} \mathrm{C}$ for 3 hours, the mixture was then filtered on celite, and the solvent was evaporated under reduced pressure. The residue was then made basic with $40 \mathrm{~mL}$ of a $10 \%$ ammonia solution and extracted with dichloromethane $(3 \times 30 \mathrm{~mL})$. The organic layer was dried over $\mathrm{Na}_{2} \mathrm{SO}_{4}$ and filtered, and the solvent was evaporated under reduced pressure. The residue was purified by column chromatography on silica with dichloromethane-methanol-ammonia $(98: 1.9: 0.1)$ as eluent to afford a colorless powder of $\mathbf{2 5 b}(414 \mathrm{mg}, 84 \%)$ : $\mathrm{mp} 21{ }^{\circ} \mathrm{C}$; IR $\left(\mathrm{CHCl}_{3}\right) \mathrm{U}_{\max }\left(\mathrm{cm}^{-1}\right): 3376-$ 3274, 2936, 1662, 1646, 1546, 1452, 1378, 1226, 1094; ${ }^{1} \mathrm{H}$ NMR $\left(300 \mathrm{MHz}, \mathrm{CDCl}_{3}\right) \delta 0.63$ $(3 \mathrm{H}, \mathrm{s}, \mathrm{H}-30), 0.84(3 \mathrm{H}, \mathrm{s}, \mathrm{H}-18), 0.87(3 \mathrm{H}, \mathrm{d}, J=6.6 \mathrm{~Hz}, \mathrm{H}-21), 0.92(3 \mathrm{H}, \mathrm{t}, J=7.4 \mathrm{~Hz}, \mathrm{H}-$ 4'), 0.93 (1H, m, H-6ß), 1.04 (1H, d, $J=3.8 \mathrm{~Hz}, \mathrm{H}-19 \alpha), 1.13$ (3H, d, $J=6.8 \mathrm{~Hz}, \mathrm{H}-5$ '), 1.22 (3H, s, H-28), $1.28(1 \mathrm{H}, \mathrm{m}, \mathrm{H}-1 \alpha), 1.31(1 \mathrm{H}, \mathrm{m}, \mathrm{H}-7 \alpha), 1.38(1 \mathrm{H}, \mathrm{m}, \mathrm{H}-7 \beta), 1.41(1 \mathrm{H}, \mathrm{m}, \mathrm{H}-$

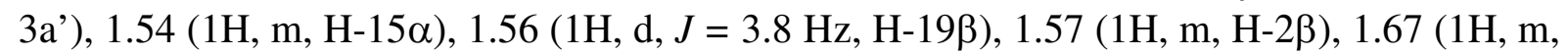

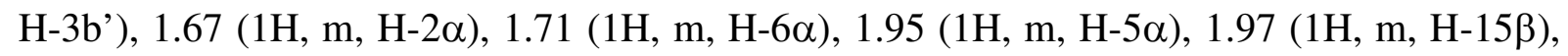
$1.97(1 \mathrm{H}, \mathrm{m}, \mathrm{H}-8 \beta), 1.97$ (1H, m, H-17 $), 2.07$ (1H, m, H-2'), $2.24\left(6 \mathrm{H}, \mathrm{s}, \mathrm{NB}-\mathrm{CH}_{3}\right), 2.31$ $(1 \mathrm{H}, \mathrm{d}, J=17.5 \mathrm{~Hz}, \mathrm{H}-12 \beta), 2.36(1 \mathrm{H}, \mathrm{d}, J=13.9 \mathrm{~Hz}, \mathrm{H}-29 \mathrm{a}), 2.41(1 \mathrm{H}, \mathrm{m}, \mathrm{H}-1 \beta), 2.46(1 \mathrm{H}$, $\mathrm{d}, J=13.9 \mathrm{~Hz}, \mathrm{H}-29 \mathrm{~b}), 2.51(1 \mathrm{H}, \mathrm{d}, J=17.5 \mathrm{~Hz}, \mathrm{H}-12 \alpha), 2.61(1 \mathrm{H}, \mathrm{dd}, J=6.6,10.9 \mathrm{~Hz}, \mathrm{H}-$

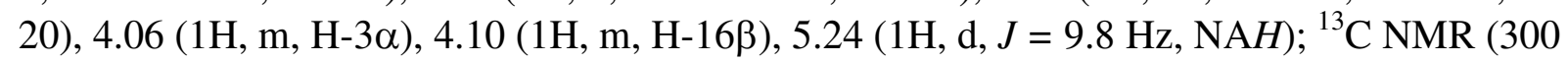
$\left.\mathrm{MHz}, \mathrm{CDCl}_{3}\right) \delta 9.9\left(\mathrm{CH}_{3}, \mathrm{C}-21\right), 12.1\left(\mathrm{CH}_{3}, \mathrm{C}-4{ }^{\prime}\right), 13.7\left(\mathrm{CH}_{3}, \mathrm{C}-30\right), 17.6\left(\mathrm{CH}_{3}, \mathrm{C}-5\right), 17.8$ $\left(\mathrm{CH}_{3}, \mathrm{C}-18\right), 18.5\left(\mathrm{CH}_{2}, \mathrm{C}-6\right), 21.0\left(\mathrm{CH}_{3}, \mathrm{C}-28\right), 24.6\left(\mathrm{CH}_{2}, \mathrm{C}-7\right), 27.5\left(\mathrm{CH}_{2}, \mathrm{C}-3\right), 27.7$ $\left(\mathrm{CH}_{2}, \mathrm{C}-1\right), 28.4\left(\mathrm{CH}_{2}, \mathrm{C}-2\right), 31.2\left(\mathrm{CH}_{2}, \mathrm{C}-19\right), 34.4$ (C, C-9), 38.1 (C, C-10), 41.7 (CH, C-8), $41.9(\mathrm{CH}, \mathrm{C}-5), 42.9\left(\mathrm{CH}_{2}, \mathrm{C}-15\right), 43.6\left(\mathrm{CH}, \mathrm{C}-2\right.$ ') $, 43.6(\mathrm{C}, \mathrm{C}-4), 44.4(\mathrm{C}, \mathrm{C}-13), 46.2\left(\mathrm{CH}_{2}\right.$, C-29), 47.1 (C, C-14), 49.7 (CH, C-3), $51.5\left(\mathrm{CH}_{2}, \mathrm{C}-12\right), 55.8(\mathrm{CH}, \mathrm{C}-17), 62.0(\mathrm{CH}, \mathrm{C}-20)$, 78.3 (CH, C-16), 176.3 (C, C-1'), 211.5 (C, C-11); ES-MS m/z $516.4[\mathrm{M}+\mathrm{H}]^{+}(100), 517.4$ (10); HRES-MS $m / z 516.4150$ (calcd for $\mathrm{C}_{31} \mathrm{H}_{54} \mathrm{~N}_{3} \mathrm{O}_{3}, 516.4165$ ). 

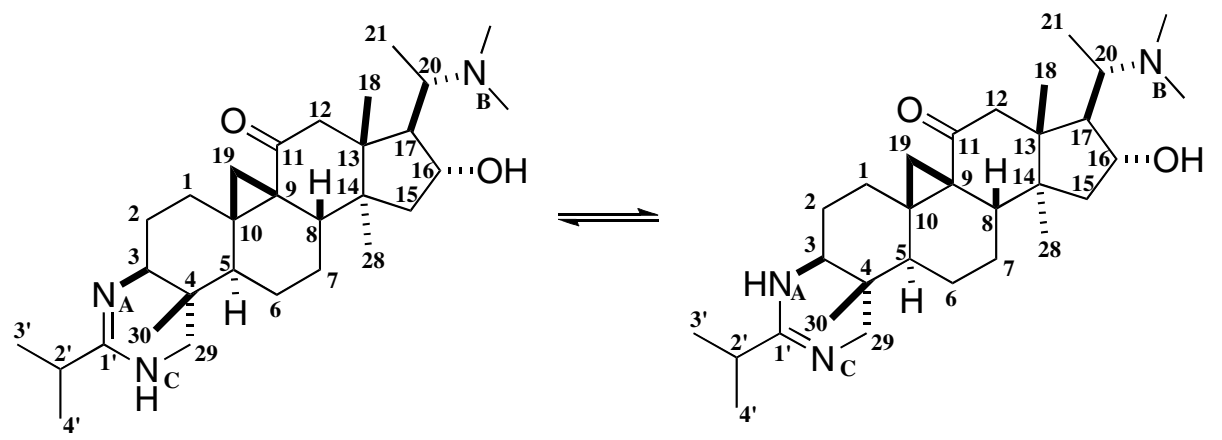

\section{(4aS,6aR,7aR,9aR,10S,11R,12aS,12bS,14aR,14bR)-10-[1-(dimethylamino)ethyl]-11-hyd roxy-3-isopropyl-9a,12a,14b-trimethyl-1,2,4a,5,6,9,9a,10,11,12,12a,12b,13,14,14a,14b- hexadecahydro-8H-cyclopenta[5,6]cyclopropa[1,8a]naphtho[2,1-f]quinazolin-8-one (26a)}

To a stirred solution of compound $\mathbf{2 5 a}$ (537 mg, $1.07 \mathrm{mmol}, 1$ equiv) in $50 \mathrm{~mL}$ of $n$-butanol was added triethylamine $\left(299 \mu \mathrm{L}, 2.14 \mathrm{mmol}, 2\right.$ equiv). After stirring at $120{ }^{\circ} \mathrm{C}$ for 16 hours, the solvent was evaporated under reduced pressure. The residue was purified by column chromatography on alumina with dichloromethane-methanol (95:5) as eluent to afford a yellow powder of 26a $(331 \mathrm{mg}, 64 \%): \operatorname{mp~} 255^{\circ} \mathrm{C}$; $[\alpha]^{23}{ }_{\mathrm{D}}=+48\left(\mathrm{c} 2.1, \mathrm{CHCl}_{3}\right)$; IR $\left(\mathrm{CHCl}_{3}\right)$ $\mathrm{U}_{\max }\left(\mathrm{cm}^{-1}\right): 3375-3141,2932,1662,1629,1530,1456,1377,1039 ;{ }^{1} \mathrm{H}$ NMR $(500 \mathrm{MHz}$, $\left.\mathrm{CDCl}_{3}\right) \delta 0.67(3 \mathrm{H}, \mathrm{s}, \mathrm{H}-30), 0.77(3 \mathrm{H}, \mathrm{s}, \mathrm{H}-18), 0.80(3 \mathrm{H}, \mathrm{d}, J=6.3 \mathrm{~Hz}, \mathrm{H}-21), 1.00(1 \mathrm{H}, \mathrm{m}$,

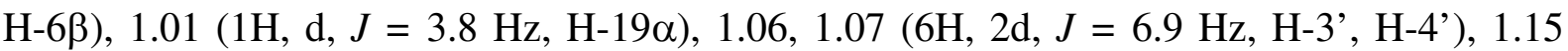
(3H, s, H-28), $1.24(1 \mathrm{H}, \mathrm{m}, \mathrm{H}-1 \alpha), 1.31(1 \mathrm{H}, \mathrm{m}, \mathrm{H}-7 \alpha), 1.37(1 \mathrm{H}, \mathrm{m}, \mathrm{H}-7 \beta), 1.42(1 \mathrm{H}, \mathrm{dd}, J=$

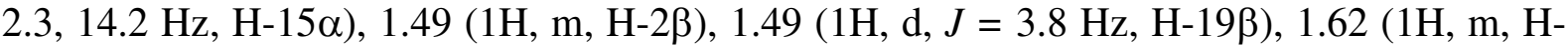

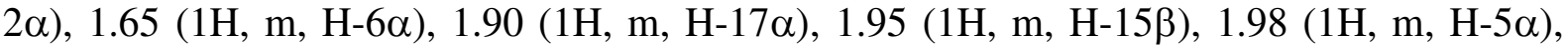
$2.03(1 \mathrm{H}, \mathrm{m}, \mathrm{H}-8 \beta), 2.18\left(6 \mathrm{H}, \mathrm{s}, \mathrm{NB}-\mathrm{CH}_{3}\right), 2.25(1 \mathrm{H}, \mathrm{d}, J=17.0 \mathrm{~Hz}, \mathrm{H}-12 \beta), 2.30(1 \mathrm{H}, \mathrm{dq}, J$ $\left.=6.6 \mathrm{~Hz}, \mathrm{H}-2^{\prime}\right), 2.31(1 \mathrm{H}, \mathrm{m}, \mathrm{H}-1 \beta), 2.42(1 \mathrm{H}, \mathrm{d}, J=17.0 \mathrm{~Hz}, \mathrm{H}-12 \alpha), 2.55(1 \mathrm{H}, \mathrm{dd}, J=6.3$, $10.8 \mathrm{~Hz}, \mathrm{H}-20), 2.92(1 \mathrm{H}, \mathrm{d}, J=13.0 \mathrm{~Hz}, \mathrm{H}-29 \mathrm{a}), 3.10(1 \mathrm{H}, \mathrm{d}, J=13.0 \mathrm{~Hz}, \mathrm{H}-29 \mathrm{~b}), 3.11(1 \mathrm{H}$, m, H-3 $\alpha), 4.02(1 \mathrm{H}, \mathrm{ddd}, J=2.3,7.6,14.2 \mathrm{~Hz}, \mathrm{H}-16 \beta), 4.23(1 \mathrm{H}, \mathrm{bs}, \mathrm{NCH} / \mathrm{NAH}$ exchanging); ${ }^{13} \mathrm{C}$ NMR $\left(125.8 \mathrm{MHz}, \mathrm{CDCl}_{3}\right) \delta 9.9\left(\mathrm{CH}_{3}, \mathrm{C}-21\right), 11.6\left(\mathrm{CH}_{3}, \mathrm{C}-30\right), 17.8\left(\mathrm{CH}_{3}\right.$, C-18), $18.2\left(\mathrm{CH}_{2}, \mathrm{C}-6\right), 20.5\left(\mathrm{CH}_{3}, \mathrm{C}-3\right.$ ', C-4'), $20.6\left(\mathrm{CH}_{3}, \mathrm{C}-28\right), 24.2\left(\mathrm{CH}_{2}, \mathrm{C}-7\right), 27.4$ $\left(\mathrm{CH}_{3}, \mathrm{C}-2\right), 27.4\left(\mathrm{CH}_{2}, \mathrm{C}-1\right), 30.3\left(\mathrm{CH}_{2}, \mathrm{C}-19\right), 34.3(\mathrm{CH}, \mathrm{C}-5), 34.8\left(\mathrm{CH}, \mathrm{C}-2{ }^{\prime}\right), 34.9(\mathrm{C}, \mathrm{C}-$ 9), 38.0 (C, C-10), 41.0 (CH, C-8), $42.7\left(\mathrm{CH}_{2}, \mathrm{C}-15\right), 44.6$ (C, C-13), 47.2 (C, C-14), 47.3 (C, $\mathrm{C}-4), 51.5\left(\mathrm{CH}_{2}, \mathrm{C}-12\right), 54.2(\mathrm{CH}, \mathrm{C}-3), 55.9(\mathrm{CH}, \mathrm{C}-17), 58.3\left(\mathrm{CH}_{2}, \mathrm{C}-29\right), 62.0(\mathrm{CH}, \mathrm{C}-20)$, 78.3 (CH, C-16), 160.6 (C, C-1'), 210.9 (C, C-11); ES-MS m/z 484.4 [M+H] ${ }^{+}(100), 485.4$ (5), 439.3 (5), $242.7[\mathrm{M}+2 \mathrm{H}]^{+}$(30), 243.2 (5); HRES-MS $\mathrm{m} / z, 484.3904$ (calcd for $\mathrm{C}_{30} \mathrm{H}_{50} \mathrm{~N}_{3} \mathrm{O}_{2}$, 484.3903). 


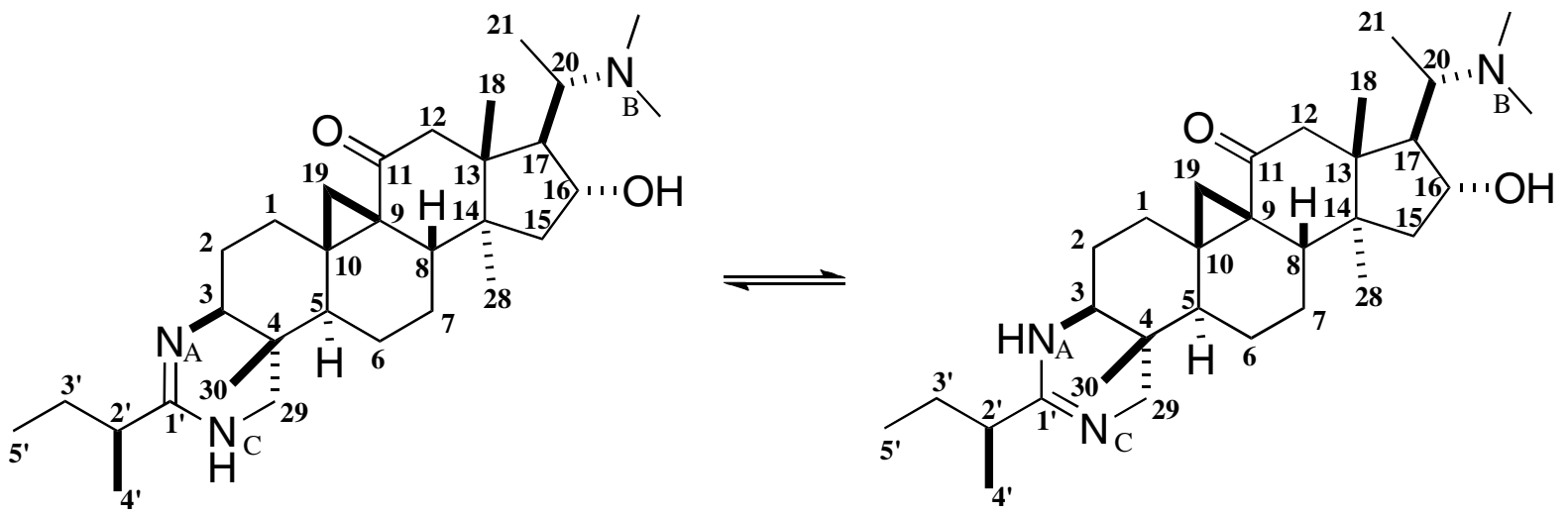

(4aS,6aR,7a $R, 9 \mathrm{a} R, 10 S, 11 R, 12 \mathrm{a} S, 12 \mathrm{~b} S, 14 \mathrm{a} R, 14 \mathrm{~b} R)-10$-[1-(dimethylamino)ethyl]-11hydroxy-9a,12a,14b-trimethyl-3-[(1S)-1-methylpropyl]-1,2,4a,5,6,9,9a,10,11,12,12a, $12 b, 13,14,14 a, 14 b$-hexadecahydro-8H-cyclopenta[5,6]cyclopropa[1,8a]naphtho $[2,1-f]$ quinazolin-8-one (26b)

To a stirred solution of compound $25 \mathbf{b}$ (300 mg, $0.58 \mathrm{mmol}, 1$ equiv) in $30 \mathrm{~mL}$ of $n$-butanol was added triethylamine $\left(162 \mu \mathrm{L}, 1.15 \mathrm{mmol}, 2\right.$ equiv). After stirring at $120{ }^{\circ} \mathrm{C}$ for 16 hours, the solvent was evaporated under reduced pressure. The residue was then made basic with 30 $\mathrm{mL}$ of a $10 \%$ ammonia solution and extracted with dichloromethane $(3 \times 40 \mathrm{~mL})$. The organic layer was dried over $\mathrm{Na}_{2} \mathrm{SO}_{4}$ and filtered, and the solvent was evaporated under reduced pressure. The residue was purified by column chromatography on alumina with dichloromethane-methanol (95:5) as eluent to afford a yellow powder of $\mathbf{2 6 \mathbf { b }}(210 \mathrm{mg}, 73 \%)$ : $\operatorname{mp} 224{ }^{\circ} \mathrm{C}$; IR $\left(\mathrm{CHCl}_{3}\right) v_{\max }\left(\mathrm{cm}^{-1}\right): 3312,2932,1665,1625,1518,1454,1376,1039 ;{ }^{1} \mathrm{H}$ NMR $\left(500 \mathrm{MHz}, \mathrm{CDCl}_{3}\right) \delta 0.69(3 \mathrm{H}, \mathrm{s}, \mathrm{H}-30), 0.77(3 \mathrm{H}, \mathrm{s}, \mathrm{H}-18), 0.80(3 \mathrm{H}, \mathrm{d}, J=6.6 \mathrm{~Hz}, \mathrm{H}-$

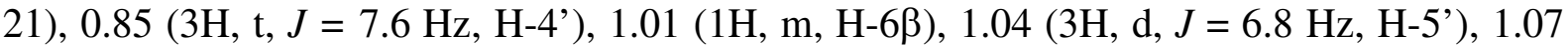
$(1 \mathrm{H}, \mathrm{d}, J=3.8 \mathrm{~Hz}, \mathrm{H}-19 \alpha), 1.14$ (3H, s, H-28), 1.19 (1H, m, H-1 $\alpha), 1.24$ (1H, m, H-7 $\alpha), 1.32$ (1H, m, H-3a'), $1.38(1 \mathrm{H}, \mathrm{m}, \mathrm{H}-7 \beta), 1.41(1 \mathrm{H}, \mathrm{bd}, J=14.0 \mathrm{~Hz}, \mathrm{H}-15 \alpha), 1.41(1 \mathrm{H}, \mathrm{m}, \mathrm{H}-2 \beta)$, $1.50(1 \mathrm{H}, \mathrm{m}, \mathrm{H}-3 \mathrm{~b}$ '), $1.55(1 \mathrm{H}, \mathrm{d}, J=3.8 \mathrm{~Hz}, \mathrm{H}-19 \beta), 1.61(1 \mathrm{H}, \mathrm{m}, \mathrm{H}-2 \alpha), 1.64(1 \mathrm{H}, \mathrm{m}, \mathrm{H}-$ $6 \alpha), 1.90(1 \mathrm{H}, \mathrm{dd}, J=7.6,10.7 \mathrm{~Hz}, \mathrm{H}-17 \alpha), 1.96(1 \mathrm{H}, \mathrm{dd}, J=10.4,14.0 \mathrm{~Hz}, \mathrm{H}-15 \beta), 2.01$

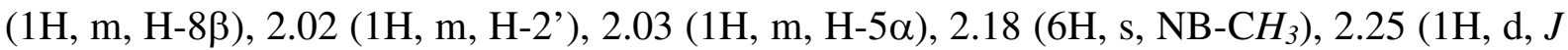
$=17.7 \mathrm{~Hz}, \mathrm{H}-12 \beta), 2.40(1 \mathrm{H}, \mathrm{m}, \mathrm{H}-1 \beta), 2.42(1 \mathrm{H}, \mathrm{d}, J=17.7 \mathrm{~Hz}, \mathrm{H}-12 \alpha), 2.55(1 \mathrm{H}, \mathrm{dd}, J=$

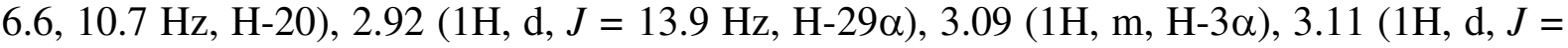
$13.9 \mathrm{~Hz}, \mathrm{H}-29 \beta), 4.02(1 \mathrm{H}, \mathrm{m}, \mathrm{H}-16 \beta), 4.23(1 \mathrm{H}, \mathrm{bs}, \mathrm{NAH}$ or $\mathrm{NCH}) ;{ }^{13} \mathrm{C}$ NMR $(125.8 \mathrm{MHz}$, $\left.\mathrm{CDCl}_{3}\right) \delta 9.9\left(\mathrm{CH}_{3}, \mathrm{C}-21\right), 11.8\left(\mathrm{CH}_{3}, \mathrm{C}-30\right), 12.2\left(\mathrm{CH}_{3}, \mathrm{C}-4\right), 17.9\left(\mathrm{CH}_{3}, \mathrm{C}-18\right), 18.2\left(\mathrm{CH}_{2}\right.$, C-6), $18.4\left(\mathrm{CH}_{3}, \mathrm{C}-5\right.$ ') $20.6\left(\mathrm{CH}_{3}, \mathrm{C}-28\right), 24.2\left(\mathrm{CH}_{2}, \mathrm{C}-7\right), 27.5\left(\mathrm{CH}_{2}, \mathrm{C}-3\right.$ ' $), 28.1\left(\mathrm{CH}_{2}, \mathrm{C}-1\right)$, $28.1\left(\mathrm{CH}_{2}, \mathrm{C}-2\right), 30.3\left(\mathrm{CH}_{2}, \mathrm{C}-19\right), 34.4(\mathrm{CH}, \mathrm{C}-5), 34.4(\mathrm{C}, \mathrm{C}-9), 38.0(\mathrm{C}, \mathrm{C}-10), 41.0(\mathrm{CH}$, C-8), 42.4 (CH, C-2'), $42.7\left(\mathrm{CH}_{2}, \mathrm{C}-15\right), 44.6$ (C, C-13), 47.2 (C, C-4), 47.3 (C, C-14), 51.5 $\left(\mathrm{CH}_{2}, \mathrm{C}-12\right), 55.5(\mathrm{CH}, \mathrm{C}-3), 55.9(\mathrm{CH}, \mathrm{C}-17), 58.1\left(\mathrm{CH}_{2}, \mathrm{C}-29\right), 62.1(\mathrm{CH}, \mathrm{C}-20), 78.3(\mathrm{CH}$, C-16), 159.8 (C, C-1'), 211.0 (C, C-11); ES-MS m/z 498.4 [M+H] ${ }^{+}$(100), 499.4 (10), 484.4 (10); HRES-MS $m / z 498.4049$ (calcd for $\mathrm{C}_{31} \mathrm{H}_{52} \mathrm{~N}_{3} \mathrm{O}_{2}, 498.4060$ ). 


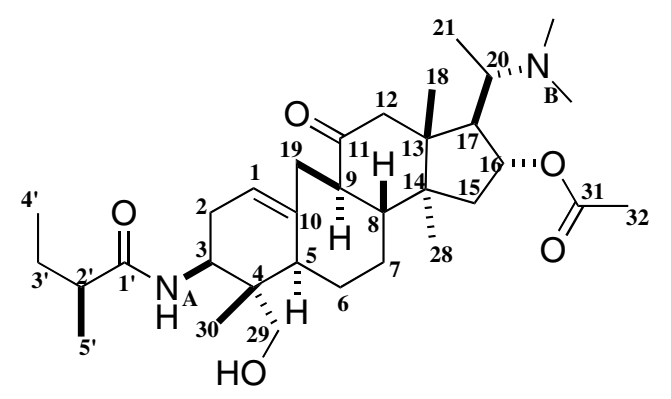

$(2 R, 3 S, 3 \mathrm{a} R, 5 \mathrm{a} R, 9 S, 10 S, 10 \mathrm{a} R, 12 \mathrm{a} R, 12 \mathrm{~b} S)-3-[1-($ dimethylamino)ethyl]-10(hydroxymethyl)-3a,10,12b-trimethyl-9- $\{[(2 S)$-2-methylbutanoyl $]$ amino $\}-5-0 x o-$

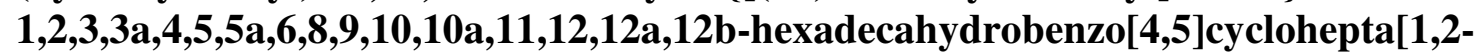
e]inden-2-yl acetate (27)

To a solution of compound $\mathbf{8 d}$ (735 mg, $1.42 \mathrm{mmol}, 1.0$ equiv) in $71 \mathrm{~mL}$ of freshly distilled dichloromethane and $21 \mathrm{~mL}$ of dried pyridine was added acetic anhydride $(202 \mu \mathrm{L}, 2.13$ mmol, 1.5 equiv). After stirring at room temperature for 16 hours, the solvent was coevaporated with 1,2-dichloroethane under reduced pressure. The residue was then made basic with $30 \mathrm{~mL}$ of a $10 \%$ ammonia solution and extracted with dichloromethane $(3 \times 20 \mathrm{~mL})$. The organic layer was dried over $\mathrm{Na}_{2} \mathrm{SO}_{4}$ and filtered, and the solvent was evaporated under reduced pressure. The residue was crystallized with acetone to afford a colorless powder of 27 $(169 \mathrm{mg})$. The mother liquor was then purified by column chromatography on alumina with dichloromethane-methanol (9:1) mixture as eluent followed by crystallisation in acetone to afford a colorless powder of $27(378 \mathrm{mg}, 90 \%)$ : $\mathrm{mp} 270{ }^{\circ} \mathrm{C}$; $[\alpha]^{23} \mathrm{D}=+53\left(\mathrm{c} 1.2 \mathrm{CHCl}_{3}\right)$; IR $\left(\mathrm{CHCl}_{3}\right) v_{\max }\left(\mathrm{cm}^{-1}\right): 3238,2962-2928,1731,1698,1632,1552,1454,1377,1243,1034 ;{ }^{1} \mathrm{H}$ NMR (300 MHz, $\left.\mathrm{CDCl}_{3}\right) \delta 0.47(3 \mathrm{H}, \mathrm{s}, \mathrm{H}-30), 0.70(3 \mathrm{H}, \mathrm{s}, \mathrm{H}-18), 0.82(3 \mathrm{H}, \mathrm{d}, J=6.2 \mathrm{~Hz}, \mathrm{H}-$

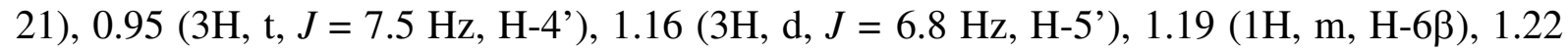
(3H, s, H-28), 1.42 (1H, m, H-7 $\alpha), 1.43$ (1H, bd, $J=14.0 \mathrm{~Hz}, \mathrm{H}-15 \alpha), 1.49$ (1H, m, H-3a'), 1.67 (1H, m, H-3b'), 1.82 (1H, m, H-7ß), $1.86(1 \mathrm{H}, \mathrm{m}, \mathrm{H}-6 \alpha), 1.90(1 \mathrm{H}, \mathrm{m}, \mathrm{H}-9 \alpha), 2.00(1 \mathrm{H}$, m, H-5 $\alpha), 2.01\left(6 \mathrm{H}, \mathrm{s}, \mathrm{NB}-\mathrm{CH}_{3}\right), 2.03(1 \mathrm{H}, \mathrm{m}, \mathrm{H}-2 \beta), 2.05(1 \mathrm{H}, \mathrm{m}, \mathrm{H}-8 \beta), 2.09(1 \mathrm{H}, \mathrm{m}, \mathrm{H}-$

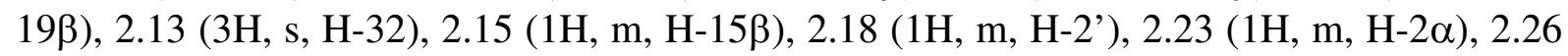
$(1 \mathrm{H}, \mathrm{dd}, J=6.1,11.0 \mathrm{~Hz}, \mathrm{H}-17 \alpha), 2.41(1 \mathrm{H}, \mathrm{d}, J=15.6 \mathrm{~Hz}, \mathrm{H}-12 \beta), 2.45(1 \mathrm{H}, \mathrm{m}, \mathrm{H}-20)$, $2.61(1 \mathrm{H}, \mathrm{d}, J=15.6 \mathrm{~Hz}, \mathrm{H}-12 \alpha), 3.16(1 \mathrm{H}, \mathrm{dd}, J=4.5,12.4 \mathrm{~Hz}, \mathrm{H}-29 \mathrm{a}), 3.35(1 \mathrm{H}, \mathrm{d}, J=$ $14.3 \mathrm{~Hz}, \mathrm{H}-19 \alpha), 3.40(1 \mathrm{H}, \mathrm{d}, J=10.9,12.4 \mathrm{~Hz}, \mathrm{H}-29 \mathrm{~b}), 4.08(1 \mathrm{H}, \mathrm{ddd}, J=6.1,9.0,10.9 \mathrm{~Hz}$, H-3 $\alpha$ ), $4.66(1 \mathrm{H}, \mathrm{dd}, J=4.5,10.9 \mathrm{~Hz}, \mathrm{OH}), 5.12(1 \mathrm{H}, \mathrm{dd}, J=6.1,8.0 \mathrm{~Hz}, \mathrm{H}-16 \beta), 5.39(1 \mathrm{H}$, $\mathrm{d}, J=8.9 \mathrm{~Hz}, \mathrm{NAH}), 5.48(1 \mathrm{H}, \mathrm{s}, \mathrm{H}-1) ;{ }^{13} \mathrm{C} \mathrm{NMR}\left(300 \mathrm{MHz}, \mathrm{CDCl}_{3}\right) \delta 9.9\left(\mathrm{CH}_{3}, \mathrm{C}-30\right), 10.0$ $\left(\mathrm{CH}_{3}, \mathrm{C}-21\right), 12.1\left(\mathrm{CH}_{3}, \mathrm{C}-4{ }^{\prime}\right), 17.6\left(\mathrm{CH}_{3}, \mathrm{C}-5\right), 17.9\left(\mathrm{CH}_{3}, \mathrm{C}-18\right), 18.0\left(\mathrm{CH}_{3}, \mathrm{C}-28\right), 21.2$ $\left(\mathrm{CH}_{3}, \mathrm{C}-32\right), 25.0\left(\mathrm{CH}_{2}, \mathrm{C}-6\right), 27.5\left(\mathrm{CH}_{2}, \mathrm{C}-3\right), 30.1\left(\mathrm{CH}_{2}, \mathrm{C}-2\right), 33.4\left(\mathrm{CH}_{2}, \mathrm{C}-7\right), 37.5\left(\mathrm{CH}_{2}\right.$, C-19), $40.1\left(\mathrm{CH}_{3}, \mathrm{NB}-\mathrm{CH}_{3}\right), 42.0(\mathrm{C}, \mathrm{C}-4), 42.7\left(\mathrm{CH}_{2}, \mathrm{C}-15\right), 43.3(\mathrm{CH}, \mathrm{C}-2$ '), $43.5(\mathrm{CH}, \mathrm{C}-$ 5), 46.2 (C, C-13), 47.3 (C, C-14), 48.0 (CH, C-3), 49.7 (CH, C-8), 49.9 (CH, C-9), 50.3 $\left(\mathrm{CH}_{2}, \mathrm{C}-12\right), 54.2(\mathrm{CH}, \mathrm{C}-17), 59.6(\mathrm{CH}, \mathrm{C}-20), 63.8\left(\mathrm{CH}_{2}, \mathrm{C}-29\right), 78.7(\mathrm{CH}, \mathrm{C}-16), 117.9$ (CH, C-1), 139.9 (C, C-10), 170.6 (C, C-31), 178.4 (C, C-1'), 211.6 (C, C-11); ES-MS m/z $559.4[\mathrm{M}+\mathrm{H}]^{+}(100), 560.4(15)$; HRES-MS $m / z 559.4150\left(\right.$ calcd for $\left.\mathrm{C}_{33} \mathrm{H}_{55} \mathrm{~N}_{2} \mathrm{O}_{5}, 559.4111\right)$. 


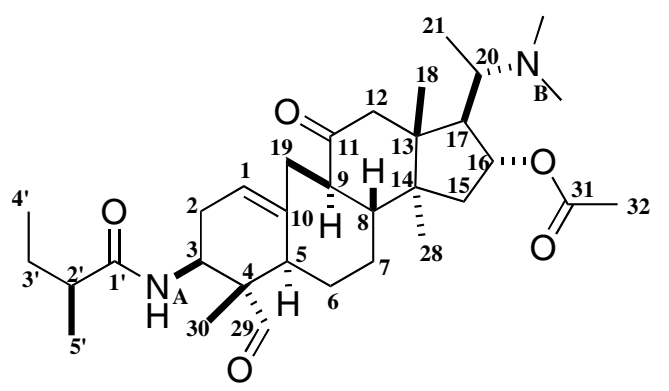

$(2 R, 3 S, 3 \mathrm{a} R, 5 \mathrm{a} R, 9 S, 10 S, 10 \mathrm{a} R, 12 \mathrm{a} R, 12 \mathrm{~b} S)-3-[1-($ dimethylamino)ethyl]-10-formyl3a,10,12b-trimethyl-9-\{[(2S)-2-methylbutanoyl]amino $\}-5-0 x 0-1,2,3,3 \mathrm{a}, 4,5,5 \mathrm{a}, 6,8,9,10$, 10a, 11,12,12a,12b-hexadecahydrobenzo[4,5]cyclohepta[1,2-e]inden-2-yl acetate (28)

To a stirred solution of compound $27(159 \mathrm{mg}, 0.28 \mathrm{mmol}, 1.0$ equiv) in $3 \mathrm{~mL}$ of dichloromethane was added Dess-Martin periodinane (162 mg, $0.37 \mathrm{mmol}, 1.3 \mathrm{equiv})$. After stirring at room temperature for 1 hour, the mixture was then made basic with $30 \mathrm{~mL}$ of a $10 \%$ ammonia solution and extracted with dichloromethane $(3 \times 25 \mathrm{~mL})$. The organic layer was dried over $\mathrm{Na}_{2} \mathrm{SO}_{4}$ and filtered, and the solvent was evaporated under reduced pressure. The residue was purified by column chromatography on silica with dichloromethane-methanol (95:5) as eluent followed by a trituration in acetone to afford a colorless powder of 28 (122 mg, 77\%): $\operatorname{mp~} 215^{\circ} \mathrm{C} ;[\alpha]^{23}{ }_{\mathrm{D}}=-15\left(\mathrm{c} 0.7 \mathrm{CHCl}_{3}\right)$; IR $\left(\mathrm{CHCl}_{3}\right) v_{\max }\left(\mathrm{cm}^{-1}\right): 3374,2965-2931$, 2790, 1726, 1697, 1666, 1649, 1529, 1452, 1373, 1245, 1030; ${ }^{1} \mathrm{H}$ NMR (300 MHz, $\left.\mathrm{CDCl}_{3}\right) \delta$ 0.68 (3H, s, H-18), 0.81 (3H, d, $J=6.4 \mathrm{~Hz}, \mathrm{H}-21), 0.82$ (3H, t, $J=7.3 \mathrm{~Hz}, \mathrm{H}-4$ '), 0.83 (3H, s, H-30), 1.06 (3H, d, $J=6.9 \mathrm{~Hz}, \mathrm{H}-5$ ') 1.21 (3H, s, H-28), $1.27(1 \mathrm{H}, \mathrm{m}, \mathrm{H}-6 \beta), 1.31(1 \mathrm{H}, \mathrm{m}$, H-7 $\alpha), 1.34$ (1H, m, H-3a'), 1.40 (1H, dd, $J=3.8,14.1 \mathrm{~Hz}, \mathrm{H}-15 \alpha), 1.55$ (1H, m, H-3b'), $1.62(1 \mathrm{H}, \mathrm{m}, \mathrm{H}-6 \alpha), 1.65(1 \mathrm{H}, \mathrm{m}, \mathrm{H}-8 \beta), 1.79(1 \mathrm{H}, \mathrm{m}, \mathrm{H}-7 \beta), 1.81(1 \mathrm{H}, \mathrm{m}, \mathrm{H}-5 \alpha), 1.85(1 \mathrm{H}$,

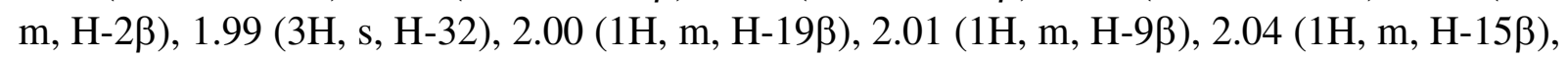
$2.12\left(6 \mathrm{H}, \mathrm{s}, \mathrm{NB}-\mathrm{CH}_{3}\right), 2.24(1 \mathrm{H}, \mathrm{dd}, J=5.8,10.8 \mathrm{~Hz}, \mathrm{H}-17 \alpha), 2.41(1 \mathrm{H}, \mathrm{d}, J=15.1 \mathrm{~Hz}, \mathrm{H}-$

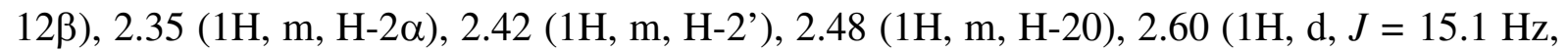
H-12 $\alpha$ ), 3.40 (1H, d, $J=14.3 \mathrm{~Hz}, \mathrm{H}-19 \alpha), 4.47$ (1H, ddd, $J=6.4,9.3,10.0 \mathrm{~Hz}, \mathrm{H}-3 \alpha), 5.10$ $(1 \mathrm{H}, \mathrm{dd}, J=6.2,7.9 \mathrm{~Hz}, \mathrm{H}-16 \beta), 5.27(1 \mathrm{H}, \mathrm{d}, J=8.5 \mathrm{~Hz}, \mathrm{NAH}), 5.54(1 \mathrm{H}, \mathrm{s}, \mathrm{H}-1), 9.47(1 \mathrm{H}$, s, H-29); ${ }^{13} \mathrm{C}$ NMR $\left(300 \mathrm{MHz}, \mathrm{CDCl}_{3}\right) \delta 6.9\left(\mathrm{CH}_{3}, \mathrm{C}-30\right), 10.1\left(\mathrm{CH}_{3}, \mathrm{C}-21\right), 11.9\left(\mathrm{CH}_{3}, \mathrm{C}-4\right.$ ' $)$, $17.4\left(\mathrm{CH}_{3}, \mathrm{C}-5\right), 18.0\left(\mathrm{CH}_{3}, \mathrm{C}-18\right), 18.0\left(\mathrm{CH}_{3}, \mathrm{C}-28\right), 21.3\left(\mathrm{CH}_{3}, \mathrm{C}-31\right), 27.1\left(\mathrm{CH}_{2}, \mathrm{C}-6\right)$, $\left.27.4\left(\mathrm{CH}_{2}, \mathrm{C}-3\right)\right), 29.3\left(\mathrm{CH}_{2}, \mathrm{C}-2\right), 33.2\left(\mathrm{CH}_{2}, \mathrm{C}-7\right), 36.9\left(\mathrm{CH}_{2}, \mathrm{C}-19\right), 40.4\left(\mathrm{CH}_{3}, \mathrm{NB}-\mathrm{CH}_{3}\right)$, $42.8\left(\mathrm{CH}_{2}, \mathrm{C}-15\right), 43.3(\mathrm{CH}, \mathrm{C}-2)$ ), $44.7(\mathrm{CH}, \mathrm{C}-5), 46.4(\mathrm{C}, \mathrm{C}-14), 47.0(\mathrm{CH}, \mathrm{C}-9), 47.4(\mathrm{C}$, C-14), $49.6(\mathrm{CH}, \mathrm{C}-3), 50.0(\mathrm{CH}, \mathrm{C}-8), 50.4\left(\mathrm{CH}_{2}, \mathrm{C}-12\right), 54.4(\mathrm{CH}, \mathrm{C}-17), 55.5(\mathrm{C}, \mathrm{C}-4)$, 59.7 (CH, C-20), 78.7 (CH, C-16), 120.1 (CH, C-1), 136.9 (C, C-10), 170.6 (C, C-31), 176.2 (C, C-1'), 204.5 (C, C-29), 211.0 (C, C-11); ES-MS m/z $557.4[\mathrm{M}+\mathrm{H}]^{+}$(100), 558.4 (15), 515.4 (15), 501.4 (5); HRES-MS $m / z 557.3957$ (calcd for $\mathrm{C}_{33} \mathrm{H}_{53} \mathrm{~N}_{2} \mathrm{O}_{5}, 557.3954$ ). 


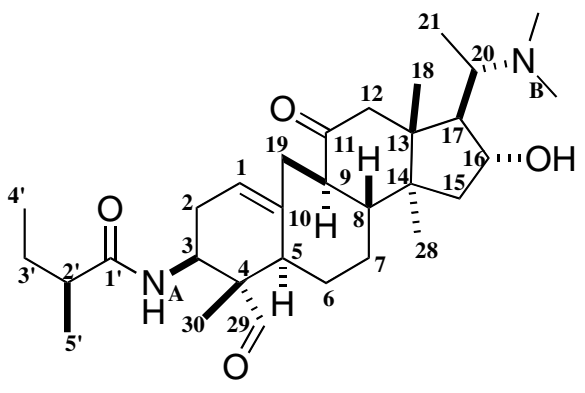

$(2 S)-N-\{(2 R, 3 S, 3 \mathrm{a} R, 5 \mathrm{a} R, 9 S, 10 S, 10 \mathrm{a} R, 12 \mathrm{a} R, 12 \mathrm{~b} S)-3-[1-($ dimethylamino)ethyl]-10-formyl2-hydroxy-3a,10,12b-trimethyl-5-oxo-1,2,3,3a,4,5,5a,6,8,9,10,10a,11,12,12a,12bhexadecahydrobenzo[4,5]cyclohepta[1,2-e]inden-9-yl\}-2-methylbutanamide

To a stirred solution of compound 28 (304 mg, $0.54 \mathrm{mmol}, 1$ equiv) in $7 \mathrm{~mL}$ of methanol heated at $40^{\circ} \mathrm{C}$ was added a suspension of sodium carbonate $(579 \mathrm{mg}, 5.46 \mathrm{mmol}, 10$ equiv) and sodium bicarbonate ( $688 \mathrm{mg}, 8.18 \mathrm{mmol}, 15$ equiv) in $11.5 \mathrm{~mL}$ of water. After stirring at $55{ }^{\circ} \mathrm{C}$ for 7 hours, the mixture was then dissolved in $40 \mathrm{~mL}$ of water and extracted with dichloromethane $(3 \times 40 \mathrm{~mL})$. The organic layer was dried over $\mathrm{Na}_{2} \mathrm{SO}_{4}$ and filtered, and the solvent was evaporated under reduced pressure. The residue was purified by column chromatography on alumina with dichloromethane-methanol $(98: 2)$ as eluent to afford a colorless powder of 29 (237 mg, 84\% in 2 steps): $\mathrm{mp} 218{ }^{\circ} \mathrm{C}$; IR $\left(\mathrm{CHCl}_{3}\right) \mathrm{u}_{\max }\left(\mathrm{cm}^{-1}\right): 3319$, 2962-2931, 2787, 1722, 1695, 1649, 1528, 1454, 1370, 1214, 1016; ${ }^{1} \mathrm{H}$ NMR $(300 \mathrm{MHz}$, $\left.\mathrm{CDCl}_{3}\right) \delta 0.62(3 \mathrm{H}, \mathrm{s}, \mathrm{H}-18), 0.76(3 \mathrm{H}, \mathrm{s}, \mathrm{H}-30), 0.78$ (3H, t, $J=7.4 \mathrm{~Hz}, \mathrm{H}-4$ '), 0.81 (3H, d, $J$ $=6.4 \mathrm{~Hz}, \mathrm{H}-21), 1.00(3 \mathrm{H}, \mathrm{d}, J=6.8 \mathrm{~Hz}, \mathrm{H}-5$ '), $1.20(3 \mathrm{H}, \mathrm{s}, \mathrm{H}-28), 1.26(1 \mathrm{H}, \mathrm{m}, \mathrm{H}-6 \beta), 1.31$

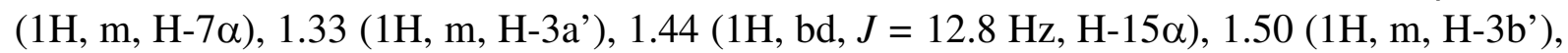
$1.58(1 \mathrm{H}, \mathrm{m}, \mathrm{H}-8 \beta), 1.73(1 \mathrm{H}, \mathrm{m}, \mathrm{H}-2 \beta), 1.77(1 \mathrm{H}, \mathrm{m}, \mathrm{H}-6 \alpha), 1.79(1 \mathrm{H}, \mathrm{m}, \mathrm{H}-7 \beta), 1.82(1 \mathrm{H}$,

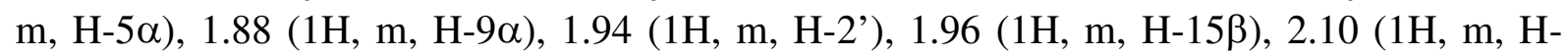
$19 \beta), 2.17\left(6 \mathrm{H}, \mathrm{s}, \mathrm{NB}-\mathrm{CH}_{3}\right), 2.29(1 \mathrm{H}, \mathrm{d}, J=15.8 \mathrm{~Hz}, \mathrm{H}-12 \beta), 2.36(1 \mathrm{H}, \mathrm{m}, \mathrm{H}-2 \alpha), 2.52(1 \mathrm{H}$, $\mathrm{d}, J=15.8 \mathrm{~Hz}, \mathrm{H}-12 \alpha), 2.55$ (1H, m, H-20), 3.34 (1H, d, $J=14.3 \mathrm{~Hz}, \mathrm{H}-19 \alpha), 4.01$ (1H, ddd, $J=2.5,8.4,8.5 \mathrm{~Hz}, \mathrm{H}-16 \beta), 4.40(1 \mathrm{H}, \mathrm{ddd}, J=6.4,7.1,12.1 \mathrm{~Hz}, \mathrm{H}-3 \alpha), 5.22(1 \mathrm{H}, \mathrm{d}, J=$ $10.0 \mathrm{~Hz}, \mathrm{NAH}), 5.48(1 \mathrm{H}, \mathrm{s}, \mathrm{H}-1), 9.42(1 \mathrm{H}, \mathrm{s}, \mathrm{H}-29) ;{ }^{13} \mathrm{C} \mathrm{NMR}\left(300 \mathrm{MHz}, \mathrm{CDCl}_{3}\right) \delta 6.8$ $\left(\mathrm{CH}_{3}, \mathrm{C}-30\right), 10.0\left(\mathrm{CH}_{3}, \mathrm{C}-21\right), 11.8\left(\mathrm{CH}_{3}, \mathrm{C}-4^{\prime}\right), 17.3\left(\mathrm{CH}_{3}, \mathrm{C}-5\right), 17.8\left(\mathrm{CH}_{3}, \mathrm{C}-18\right), 18.8$ $\left(\mathrm{CH}_{3}, \mathrm{C}-28\right), 27.1\left(\mathrm{CH}_{2}, \mathrm{C}-6\right), 27.3\left(\mathrm{CH}_{2}, \mathrm{C}-3\right), 29.2\left(\mathrm{CH}_{2}, \mathrm{C}-2\right), 33.2\left(\mathrm{CH}_{2}, \mathrm{C}-7\right), 37.0\left(\mathrm{CH}_{2}\right.$, C-19), $42.4\left(\mathrm{CH}_{2}, \mathrm{C}-15\right), 43.2(\mathrm{CH}, \mathrm{C}-2$ '), 44.6 (CH, C-8), 46.2 (C, C-13), 46.8 (CH, C-5), $47.0(\mathrm{C}, \mathrm{C}-14), 49.6(\mathrm{CH}, \mathrm{C}-3), 50.0(\mathrm{CH}, \mathrm{C}-12), 50.3(\mathrm{CH}, \mathrm{C}-9), 55.4(\mathrm{C}, \mathrm{C}-4), 55.4(\mathrm{CH}, \mathrm{C}-$ 17), 62.0 (CH, C-20), 78.3 (CH, C-16), 119.9 (CH, C-1), 136.8 (C, C-10), 176.1 (C, C-1'), 204.5 (C, C-29), 211.4 (C, C-11); ES-MS m/z $515.3[\mathrm{M}+\mathrm{H}]^{+}$(100), 516.4 (45), 501.4 (5); HRES-MS $m / z 515.3844$ (calcd for $\mathrm{C}_{31} \mathrm{H}_{51} \mathrm{~N}_{2} \mathrm{O}_{4}, 515.3849$ ). 


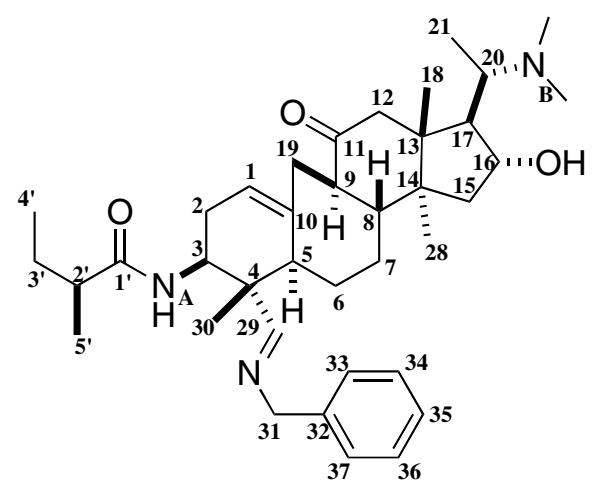

\section{Compound (30)}

To a stirred suspension of compound 29 (200 $\mathrm{mg}, 0.39 \mathrm{mmol}, 1.0$ equiv) and $160 \mathrm{mg}$ of anhydrous magnesium sulfate in $2 \mathrm{~mL}$ of freshly distilled dichloromethane was added benzylamine $\left(55 \mu \mathrm{L}, 0.50 \mathrm{mmol}, 1.3\right.$ equiv). After stirring at $55{ }^{\circ} \mathrm{C}$ for 7 hours, the mixture was then filtered on celite, and the solvent was evaporated under reduced pressure to afford a colorless powder of $\mathbf{3 0}\left(272 \mathrm{mg}\right.$, 99\%): IR $\left(\mathrm{CHCl}_{3}\right) \mathrm{U}_{\max }\left(\mathrm{cm}^{-1}\right)$ : 3309, 2929, 1728, 1695, 1649, $1532,1451,1375,1227,1091 ;{ }^{1} \mathrm{H}$ NMR $\left(300 \mathrm{MHz}, \mathrm{CDCl}_{3}\right) \delta 0.61(3 \mathrm{H}, \mathrm{s}, \mathrm{H}-18), 0.75(3 \mathrm{H}, \mathrm{t}$, $\left.J=7.4 \mathrm{~Hz}, \mathrm{H}-4^{\prime}\right), 0.80(3 \mathrm{H}, \mathrm{d}, J=6.4 \mathrm{~Hz}, \mathrm{H}-21), 0.86(3 \mathrm{H}, \mathrm{s}, \mathrm{H}-30), 0.96(3 \mathrm{H}, \mathrm{d}, J=7.0 \mathrm{~Hz}$,

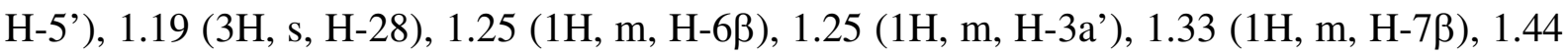
$(1 \mathrm{H}, \mathrm{m}, \mathrm{H}-15 \alpha), 1.47\left(1 \mathrm{H}, \mathrm{m}, \mathrm{H}-3 \mathrm{~b}^{\prime}\right), 1.58(1 \mathrm{H}, \mathrm{m}, \mathrm{H}-8 \beta), 1.69(1 \mathrm{H}, \mathrm{m}, \mathrm{H}-6 \alpha), 1.77(1 \mathrm{H}, \mathrm{m}$, $\mathrm{H}-2 \beta), 1.86(1 \mathrm{H}, \mathrm{m}, \mathrm{H}-7 \beta), 1.88$ (1H, m, H-5 $\alpha), 1.88(1 \mathrm{H}, \mathrm{m}, \mathrm{H}-9 \alpha), 1.92(1 \mathrm{H}, \mathrm{m}, \mathrm{H}-15 \beta)$, $1.94\left(1 \mathrm{H}, \mathrm{m}, \mathrm{H}-2^{\prime}\right), 1.95(1 \mathrm{H}, \mathrm{m}, \mathrm{H}-17 \alpha), 2.01(1 \mathrm{H}, \mathrm{m}, \mathrm{H}-19 \beta), 2.06$ (6H, s, NB-CH $\left.{ }_{3}\right), 2.28$ $(1 \mathrm{H}, \mathrm{d}, J=16.0 \mathrm{~Hz}, \mathrm{H}-12 \beta), 2.33(1 \mathrm{H}, \mathrm{m}, \mathrm{H}-2 \alpha), 2.51(1 \mathrm{H}, \mathrm{d}, J=16.0 \mathrm{~Hz}, \mathrm{H}-12 \alpha), 2.54(1 \mathrm{H}$, m, H-20), $3.30(1 \mathrm{H}, \mathrm{d}, J=14.5 \mathrm{~Hz}, \mathrm{H}-19 \alpha), 4.02(1 \mathrm{H}, \mathrm{ddd}, J=2.0,7.5,7.9 \mathrm{~Hz}, \mathrm{H}-16 \beta), 4.19$ (1H, ddd, $J=6.2,7.0,13.0 \mathrm{~Hz}, \mathrm{H}-3 \alpha), 4.44,4.56(2 \mathrm{H}, 2 \mathrm{~d}, J=13.4 \mathrm{~Hz}, \mathrm{H}-31), 5.22(1 \mathrm{H}, \mathrm{d}, J$ $=9.0 \mathrm{~Hz}, \mathrm{NAH}), 5.47(1 \mathrm{H}, \mathrm{s}, \mathrm{H}-1), 7.15-7.28(5 \mathrm{H}, \mathrm{m}, \mathrm{HAr}=33,34,35,36,37), 7.56(1 \mathrm{H}, \mathrm{s}$, $\mathrm{H}-29) ;{ }^{13} \mathrm{C}$ NMR $\left(300 \mathrm{MHz}, \mathrm{CDCl}_{3}\right) \delta 9.3\left(\mathrm{CH}_{3}, \mathrm{C}-30\right), 10.0\left(\mathrm{CH}_{3}, \mathrm{C}-21\right), 11.8\left(\mathrm{CH}_{3}, \mathrm{C}-4\right)$, 17.2 ( $\mathrm{CH}_{3}, \mathrm{C}-5$ ') $\left., 17.8\left(\mathrm{CH}_{3}, \mathrm{C}-18\right), 18.9\left(\mathrm{CH}_{3}, \mathrm{C}-28\right), 26.2\left(\mathrm{CH}_{2}, \mathrm{C}-6\right), 27.2\left(\mathrm{CH}_{2}, \mathrm{C}-3\right)\right)$, $29.7\left(\mathrm{CH}_{2}, \mathrm{C}-2\right), 33.2\left(\mathrm{CH}_{2}, \mathrm{C}-7\right), 37.1\left(\mathrm{CH}_{2}, \mathrm{C}-19\right), 42.5\left(\mathrm{CH}_{2}, \mathrm{C}-15\right), 43.3\left(\mathrm{CH}, \mathrm{C}-2^{\prime}\right), 46.2$ (C, C-13), 47.7 (CH, C-8), 47.9 (CH, C-5), 47.0 (C, C-14), 49.6 (CH, C-3), $50.0\left(\mathrm{CH}_{2}, \mathrm{C}-12\right)$, $50.3(\mathrm{CH}, \mathrm{C}-9), 55.4(\mathrm{CH}, \mathrm{C}-17), 59.1(\mathrm{C}, \mathrm{C}-4), 62.0(\mathrm{CH}, \mathrm{C}-20), 64.7\left(\mathrm{CH}_{2}, \mathrm{C}-31\right), 78.3$ (CH, C-16), 119.9 (CH, C-1), 126.7 (CH, C-35), 127.1, 128.4 (CH, C-33, C-34, C-36, C-37), 137.4 (C, C-10), 139.5 (C, C-32), 171.1 (CH, C-29), 175.1 (C, C-1'), 211.4 (C, C-11); ES-MS $\mathrm{m} / \mathrm{z} 604.4[\mathrm{M}+\mathrm{H}]^{+}$(100), 605.4 (10), 646.5 (5); HRES-MS m/z 604.4460 (calcd for $\mathrm{C}_{38} \mathrm{H}_{58} \mathrm{~N}_{3} \mathrm{O}_{3}, 604.4478$ ). 


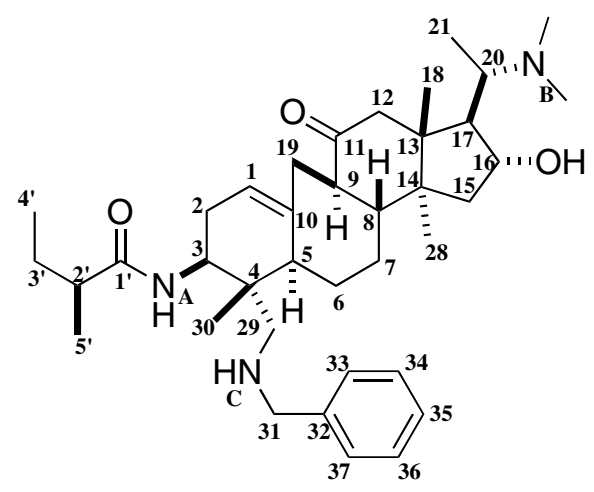

\section{Compund (31)}

To a stirred solution of crude compound $30(272 \mathrm{mg}, 0.39 \mathrm{mmol}, 1.00$ equiv) in $2 \mathrm{~mL}$ of methanol was added sodium cyanoborohydride (36 mg, $0.54 \mathrm{mmol}, 1.40$ equiv) and glacial acetic acid ( $26 \mu \mathrm{L}, 0.45 \mathrm{mmol}, 1.15$ equiv). After stirring at room temperature for 3 hours, the mixture was then made basic with $40 \mathrm{~mL}$ of a $10 \%$ ammonia solution and extracted with dichloromethane $(3 \times 40 \mathrm{~mL})$. The organic layer was dried over $\mathrm{Na}_{2} \mathrm{SO}_{4}$ and filtered, and the solvent was evaporated under reduced pressure. The residue was purified by column chromatography on alumina with dichloromethane-methanol $(98: 2)$ as eluent to afford a colorless powder of $\mathbf{3 1}$ (173 mg, 73\% in 2 steps): IR $\left(\mathrm{CHCl}_{3}\right) \mathrm{U}_{\max }\left(\mathrm{cm}^{-1}\right): 3313,2961,1695$, 1644, 1530, 1452, 1377, 1237, 1095; ${ }^{1} \mathrm{H}$ NMR (300 MHz, $\left.\mathrm{CDCl}_{3}\right) \delta 0.45$ (3H, s, H-30), 0.61 $(3 \mathrm{H}, \mathrm{s}, \mathrm{H}-18), 0.80(3 \mathrm{H}, \mathrm{d}, J=6.4 \mathrm{~Hz}, \mathrm{H}-21), 0.86(3 \mathrm{H}, \mathrm{t}, J=7.4 \mathrm{~Hz}, \mathrm{H}-4$ ') 1.06 (3H, d, $J=$

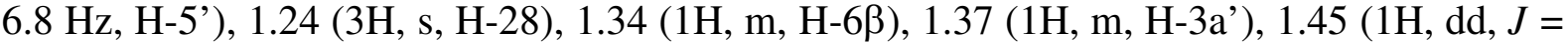

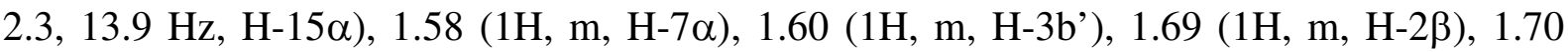
(1H, m, H-6 $\alpha), 1.72(1 \mathrm{H}, \mathrm{m}, \mathrm{H}-8 \beta), 1.82(1 \mathrm{H}, \mathrm{m}, \mathrm{H}-19 \beta), 1.88(1 \mathrm{H}, \mathrm{m}, \mathrm{H}-5 \alpha), 1.92(1 \mathrm{H}, \mathrm{m}$,

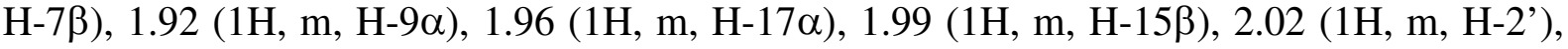
$2.17\left(6 \mathrm{H}, \mathrm{s}, \mathrm{NB}-\mathrm{CH}_{3}\right), 2.20(1 \mathrm{H}, \mathrm{d}, J=12.5 \mathrm{~Hz}, \mathrm{H}-29 \mathrm{a}), 2.27(1 \mathrm{H}, \mathrm{d}, J=15.6 \mathrm{~Hz}, \mathrm{H}-12 \beta)$, $2.32(1 \mathrm{H}, \mathrm{d}, J=12.5 \mathrm{~Hz}, \mathrm{H}-29 \mathrm{~b}), 2.33(1 \mathrm{H}, \mathrm{m}, \mathrm{H}-2 \alpha), 2.52(1 \mathrm{H}, \mathrm{d}, J=15.6 \mathrm{~Hz}, \mathrm{H}-12 \alpha), 2.62$ $(1 \mathrm{H}, \mathrm{m}, \mathrm{H}-20), 3.23(1 \mathrm{H}, \mathrm{d}, J=14.1 \mathrm{~Hz}, \mathrm{H}-19 \alpha), 3.48,3.78(2 \mathrm{H}, 2 \mathrm{~d}, J=13.6 \mathrm{~Hz}, \mathrm{H}-31)$, $4.02(2 \mathrm{H}, \mathrm{m}, \mathrm{H}-3 \alpha), 4.02(1 \mathrm{H}, \mathrm{m}, \mathrm{H}-16 \beta), 5.23(1 \mathrm{H}, \mathrm{d}, J=9.6 \mathrm{~Hz}, \mathrm{NAH}), 5.37(1 \mathrm{H}, \mathrm{s}, \mathrm{H}-1)$, 7.12-7.27 (5H, m, HAr=33, 34, 35, 36, 37); $\left.{ }^{13} \mathrm{C} \mathrm{NMR} \mathrm{(300} \mathrm{MHz,} \mathrm{CDCl}_{3}\right) \delta 10.0\left(\mathrm{CH}_{3}, \mathrm{C}-21\right)$, $12.0\left(\mathrm{CH}_{3}, \mathrm{C}-4\right.$ '), $12.4\left(\mathrm{CH}_{3}, \mathrm{C}-30\right), 17.6\left(\mathrm{CH}_{3}, \mathrm{C}-18\right), 17.7\left(\mathrm{CH}_{3}, \mathrm{C}-5\right)$ ') $19.0\left(\mathrm{CH}_{3}, \mathrm{C}-28\right)$, $25.2\left(\mathrm{CH}_{2}, \mathrm{C}-6\right), 27.4\left(\mathrm{CH}_{2}, \mathrm{C}-3{ }^{\prime}\right), 30.7\left(\mathrm{CH}_{2}, \mathrm{C}-2\right), 33.3\left(\mathrm{CH}_{2}, \mathrm{C}-7\right), 37.5\left(\mathrm{CH}_{2}, \mathrm{C}-19\right), 40.5$ (C, C-4), $42.5\left(\mathrm{CH}_{2}, \mathrm{C}-15\right), 43.7$ (CH, C-2'), 44.7 (CH, C-5), 46.3 (C, C-13), 47.1 (C, C-14), 48.0 (CH, C-3), 49.7 (CH, C-8), $50.1\left(\mathrm{CH}_{2}, \mathrm{C}-12\right), 50.3(\mathrm{CH}, \mathrm{C}-9), 52.6\left(\mathrm{CH}_{2}, \mathrm{C}-29\right), 54.3$ $\left(\mathrm{CH}_{2}, \mathrm{C}-31\right), 55.4(\mathrm{CH}, \mathrm{C}-17), 62.1(\mathrm{CH}, \mathrm{C}-20), 78.4(\mathrm{CH}, \mathrm{C}-16), 118.3(\mathrm{CH}, \mathrm{C}-1), 126.6$ (CH, C-35), 128.0, 128.4 (CH, C-33, C-34, C-36, C-37), 139.8 (C, C-10), 141.2 (C, C-32), 176.4 (C, C-1'), 212.0 (C, C-11); ES-MS m/z 606.4 [M+H] ${ }^{+}(100), 607.5$ (20); HRES-MS $m / z$ 606.4636 (calcd for $\mathrm{C}_{38} \mathrm{H}_{60} \mathrm{~N}_{3} \mathrm{O}_{3}, 606.4635$ ). 


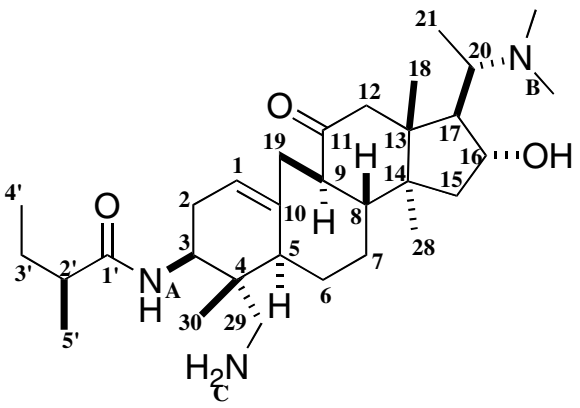

(2S)-N-\{(2R,3S,3aR,5aR,9S,10R,10aR,12aR,12bS)-10-(aminomethyl)-3-[1-(dimethylami no)ethyl]-2-hydroxy-3a,10,12b-trimethyl-5-oxo-

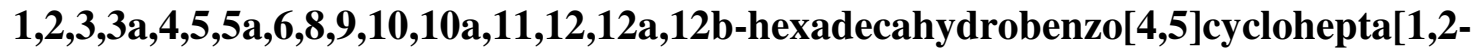
e]inden-9-yl\}-2-methylbutanamide

(32)

To a stirred suspension of compound 31 (296 mg, $0.49 \mathrm{mmol}, 1$ equiv) in $10 \mathrm{~mL}$ of dreshly distilled methanol was added palladium at $30 \%$ on carbon $(148 \mathrm{mg}, 50 \%$ by weight) and ammonium formate (159 mg, $2.43 \mathrm{mmol}, 5$ equiv). After stirring at $40{ }^{\circ} \mathrm{C}$ for 3 hours, the mixture was then filtered on celite, and the solvent was evaporated under reduced pressure. The residue was then made basic with $40 \mathrm{~mL}$ of a $10 \%$ ammonia solution and extracted with dichloromethane $(3 \times 30 \mathrm{~mL})$. The organic layer was dried over $\mathrm{Na}_{2} \mathrm{SO}_{4}$ and filtered, and the solvent was evaporated under reduced pressure. The residue was purified by column chromatography on silica with dichloromethane-methanol-ammonia (90:9.9:0.1) as eluent to afford a colorless powder of 32 (90 mg, 36\%): $\mathrm{mp} 161{ }^{\circ} \mathrm{C}$; IR $\left(\mathrm{CHCl}_{3}\right) \mathrm{U}_{\max }\left(\mathrm{cm}^{-1}\right): 3296$, 2961-2931, 1694, 1644, 1537, 1460, 1368, 1226, 1094; ${ }^{1} \mathrm{H}$ NMR $\left(300 \mathrm{MHz}, \mathrm{CDCl}_{3}\right) \delta 0.50$ $(3 \mathrm{H}, \mathrm{s}, \mathrm{H}-30), 0.62(3 \mathrm{H}, \mathrm{s}, \mathrm{H}-18), 0.81(3 \mathrm{H}, \mathrm{d}, J=6.6 \mathrm{~Hz}, \mathrm{H}-21), 0.86(3 \mathrm{H}, \mathrm{t}, J=7.4 \mathrm{~Hz}, \mathrm{H}-$ 4'), 1.05 (3H, d, J = 6.8 Hz, H-5'), 1.21 (3H, s, H-28), 1.30 (1H, m, H-6ß), 1.35 (1H, m, H-

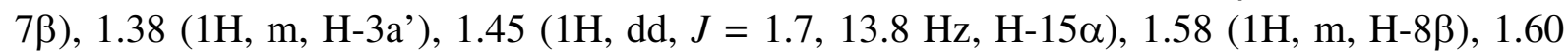

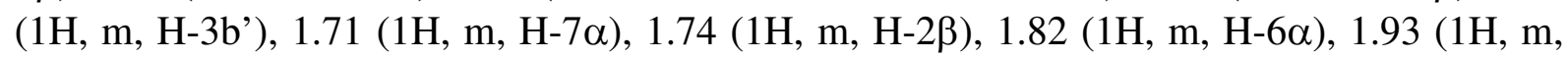

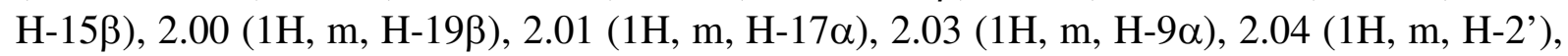
$2.19\left(6 \mathrm{H}, \mathrm{s}, \mathrm{NB}-\mathrm{CH}_{3}\right), 2.28(1 \mathrm{H}, \mathrm{d}, J=15.3 \mathrm{~Hz}, \mathrm{H}-12 \beta), 2.27(1 \mathrm{H}, \mathrm{m}, \mathrm{H}-2 \alpha), 2.46(1 \mathrm{H}, \mathrm{d}, J=$ $12.5 \mathrm{~Hz}, \mathrm{H}-29 \mathrm{a}), 2.50(1 \mathrm{H}, \mathrm{d}, J=15.3 \mathrm{~Hz}, \mathrm{H}-12 \alpha), 2.53(1 \mathrm{H}, \mathrm{m}, \mathrm{H}-5 \alpha), 2.56(1 \mathrm{H}, \mathrm{d}, J=13.0$

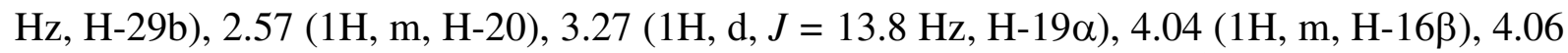
$(1 \mathrm{H}, \mathrm{m}, \mathrm{H}-3 \alpha), 5.30(1 \mathrm{H}, \mathrm{d}, J=9.6 \mathrm{~Hz}, \mathrm{NAH}), 5.41(1 \mathrm{H}, \mathrm{s}, \mathrm{H}-1) ;{ }^{13} \mathrm{C}$ NMR $(300 \mathrm{MHz}$, $\left.\mathrm{CDCl}_{3}\right) \delta 10.0\left(\mathrm{CH}_{3}, \mathrm{C}-21\right), 12.0\left(\mathrm{CH}_{3}, \mathrm{C}-4^{\prime}\right), 12.4\left(\mathrm{CH}_{3}, \mathrm{C}-30\right), 17.6\left(\mathrm{CH}_{3}, \mathrm{C}-5\right), 17.8\left(\mathrm{CH}_{3}\right.$, C-18), $\left.19.0\left(\mathrm{CH}_{3}, \mathrm{C}-28\right), 25.0\left(\mathrm{CH}_{2}, \mathrm{C}-6\right), 27.4\left(\mathrm{CH}_{2}, \mathrm{C}-3\right)\right), 30.7\left(\mathrm{CH}_{2}, \mathrm{C}-2\right), 33.4\left(\mathrm{CH}_{2}, \mathrm{C}-\right.$ 7), $37.5\left(\mathrm{CH}_{2}, \mathrm{C}-19\right), 41.1(\mathrm{C}, \mathrm{C}-4), 42.5\left(\mathrm{CH}_{2}, \mathrm{C}-15\right), 43.6(\mathrm{CH}, \mathrm{C}-2$ ') $, 43.9(\mathrm{CH}, \mathrm{C}-5), 45.6$ $\left(\mathrm{CH}_{2}, \mathrm{C}-29\right), 46.3(\mathrm{C}, \mathrm{C}-13), 47.1(\mathrm{C}, \mathrm{C}-14), 47.2(\mathrm{CH}, \mathrm{C}-3), 49.8\left(\mathrm{CH}_{2}, \mathrm{C}-12\right), 50.0(\mathrm{CH}, \mathrm{C}-$ 8), $50.2(\mathrm{CH}, \mathrm{C}-9), 55.3(\mathrm{CH}, \mathrm{C}-17), 62.1(\mathrm{CH}, \mathrm{C}-20), 78.2(\mathrm{CH}, \mathrm{C}-16), 118.8(\mathrm{CH}, \mathrm{C}-1)$, 139.2 (C, C-10), 176.8 (C, C-1'), 211.8 (C, C-11); ES-MS m/z $516.4[\mathrm{M}+\mathrm{H}]^{+}(100), 517.5$ (10), 498.4 (10); HRES-MS $m / z 516.4144$ (calcd for $\mathrm{C}_{31} \mathrm{H}_{54} \mathrm{~N}_{3} \mathrm{O}_{3}, 516.4165$ ). 


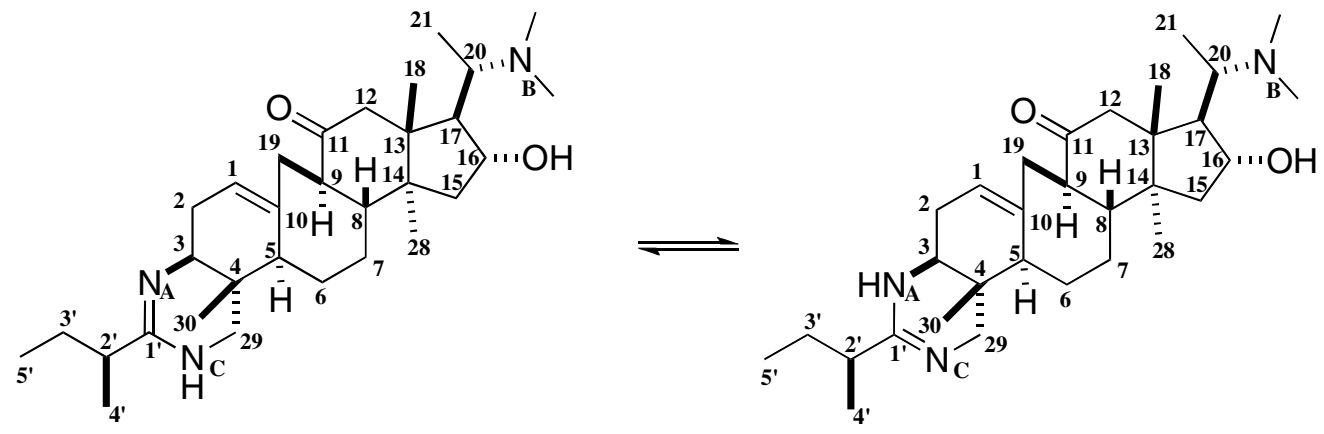

$(4 \mathrm{a} S, 7 \mathrm{a} R, 9 \mathrm{a} R, 10 S, 11 R, 12 \mathrm{a} S, 12 \mathrm{~b} R, 14 \mathrm{a} R, 14 \mathrm{~b} R)-10$-[1-(dimethylamino)ethyl]-11-hydroxy -9a,12a,14b-trimethyl-3-[(1S)-1-methylpropyl]-2,4a,5,7,7a,9,9a,10,11,12,12a,12b, 13,14, 14a,14b-hexadecahydroindeno[ $\left.5^{\prime}, 4^{\prime}: 4,5\right]$ cyclohepta[1,2-f]quinazolin-8(1H)-one (33)

To a stirred solution of compound 32 (61 mg, $0.12 \mathrm{mmol}, 1$ equiv) in $30 \mathrm{~mL}$ of $n$-butanol was added triethylamine ( $37 \mu \mathrm{L}, 0.26 \mathrm{mmol}, 2$ equiv). After stirring at $120{ }^{\circ} \mathrm{C}$ for 16 hours, the solvent was evaporated under reduced pressure. The residue was then made basic with $30 \mathrm{~mL}$ of a 10\% ammonia solution and extracted with dichloromethane $(3 \times 40 \mathrm{~mL})$. The organic layer was dried over $\mathrm{Na}_{2} \mathrm{SO}_{4}$ and filtered, and the solvent was evaporated under reduced pressure. The residue was purified by column chromatography on alumina with dichloromethanemethanol (95:5) as eluent to afford a yellow powder of $\mathbf{3 3}(29 \mathrm{mg}, 49 \%)$ : $\mathrm{mp} 225{ }^{\circ} \mathrm{C}$; IR $\left(\mathrm{CHCl}_{3}\right) \cup_{\max }\left(\mathrm{cm}^{-1}\right): 3315,2961-2932,1694,1627,1518,1459,1381,1039 ;{ }^{1} \mathrm{H}$ NMR (500 $\left.\mathrm{MHz}, \mathrm{CDCl}_{3}\right) \delta 0.63(3 \mathrm{H}, \mathrm{s}, \mathrm{H}-18), 0.67(3 \mathrm{H}, \mathrm{s}, \mathrm{H}-30), 0.80(3 \mathrm{H}, \mathrm{d}, J=6.3 \mathrm{~Hz}, \mathrm{H}-21), 0.85$ $\left(3 \mathrm{H}, \mathrm{t}, J=7.5 \mathrm{~Hz}, \mathrm{H}-4^{\prime}\right), 1.04\left(3 \mathrm{H}, \mathrm{d}, J=6.9 \mathrm{~Hz}, \mathrm{H}-5^{\prime}\right), 1.17(3 \mathrm{H}, \mathrm{s}, \mathrm{H}-28), 1.26(1 \mathrm{H}, \mathrm{m}, \mathrm{H}-$

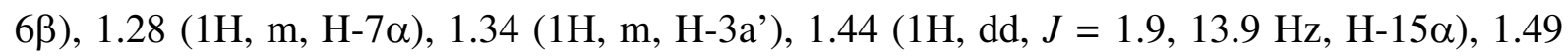

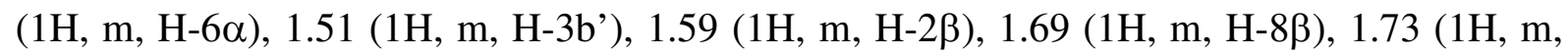
$\mathrm{H}-2 \alpha), 1.85(1 \mathrm{H}, \mathrm{m}, \mathrm{H}-5 \alpha), 1.91(1 \mathrm{H}, \mathrm{m}, \mathrm{H}-7 \beta), 1.91(1 \mathrm{H}, \mathrm{m}, \mathrm{H}-15 \beta), 1.93$ (1H, dd, $J=6.9$,

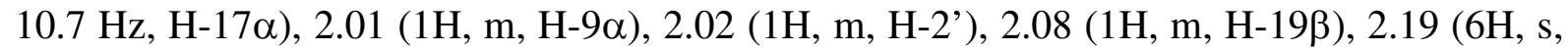
NB-CH $H_{3}, 2.21(1 \mathrm{H}, \mathrm{m}, \mathrm{H}-2 \alpha), 2.28(1 \mathrm{H}, \mathrm{d}, J=15.8 \mathrm{~Hz}, \mathrm{H}-12 \beta), 2.48(1 \mathrm{H}, \mathrm{d}, J=15.8 \mathrm{~Hz}$, $\mathrm{H}-12 \alpha), 2,57$ (1H, dd, $J=6.3,10.7 \mathrm{~Hz}, \mathrm{H}-20), 2.95(1 \mathrm{H}, \mathrm{d}, J=12.9 \mathrm{~Hz}, \mathrm{H}-29 \alpha), 3.24(1 \mathrm{H}, \mathrm{d}$, $J=15.8 \mathrm{~Hz}, \mathrm{H}-19 \alpha), 3.25(1 \mathrm{H}, \mathrm{d}, J=12.9 \mathrm{~Hz}, \mathrm{H}-29 \beta), 4.04(1 \mathrm{H}, \mathrm{dd}, J=6.9,7.3 \mathrm{~Hz}, \mathrm{H}-$ $16 \beta), 4.17(1 \mathrm{H}, \mathrm{bs}, \mathrm{NAH}$ or $\mathrm{NCH}), 5.47(1 \mathrm{H}, \mathrm{s}, \mathrm{H}-1) ;{ }^{13} \mathrm{C} \mathrm{NMR}\left(125.8 \mathrm{MHz}, \mathrm{CDCl}_{3}\right) \delta 10.0$ $\left(\mathrm{CH}_{3}, \mathrm{C}-21\right), 10.9\left(\mathrm{CH}_{3}, \mathrm{C}-30\right), 12.2\left(\mathrm{CH}_{3}, \mathrm{C}-4^{\prime}\right), 17.8\left(\mathrm{CH}_{3}, \mathrm{C}-18\right), 18.6\left(\mathrm{CH}_{3}, \mathrm{C}-5\right), 18.8$ $\left(\mathrm{CH}_{3}, \mathrm{C}-28\right), 25.0\left(\mathrm{CH}_{2}, \mathrm{C}-6\right), 28.2\left(\mathrm{CH}_{2}, \mathrm{C}-3\right.$ ') $, 29.7\left(\mathrm{CH}_{2}, \mathrm{C}-2\right), 33.1(\mathrm{C}, \mathrm{C}-4), 33.8\left(\mathrm{CH}_{2}, \mathrm{C}-\right.$ 7), $37.5\left(\mathrm{CH}_{2}, \mathrm{C}-19\right), 42.4\left(\mathrm{CH}, \mathrm{C}-2\right.$ ') $, 42.5\left(\mathrm{CH}_{2}, \mathrm{C}-15\right), 46.2$ (C, C-13), 47.1 (C, C-14), 49.8 $(\mathrm{CH}, \mathrm{C}-5), 50.1\left(\mathrm{CH}_{2}, \mathrm{C}-12\right), 50.4(\mathrm{CH}, \mathrm{C}-9), 50.8(\mathrm{CH}, \mathrm{C}-3), 51.0(\mathrm{CH}, \mathrm{C}-8), 54.2\left(\mathrm{CH}_{2}, \mathrm{C}-\right.$ 29), $55.5(\mathrm{CH}, \mathrm{C}-17), 62.0(\mathrm{CH}, \mathrm{C}-20), 78.4(\mathrm{CH}, \mathrm{C}-16), 120.1(\mathrm{CH}, \mathrm{C}-1), 138.5(\mathrm{C}, \mathrm{C}-10)$, 159.9 (C, C-1'), 211.9 (C, C-11); ES-MS m/z 498.4 [M+H] ${ }^{+}$(100), 499.4 (10); HRES-MS m/z 498.4043 (calcd for $\mathrm{C}_{31} \mathrm{H}_{52} \mathrm{~N}_{3} \mathrm{O}_{2}, 498.4060$ ). 


\begin{tabular}{|c|c|c|c|c|c|c|c|c|c|}
\hline \multicolumn{10}{|c|}{ Table of Elemental analysis } \\
\hline & & \multicolumn{2}{|c|}{$\mathbf{C}$} & \multicolumn{2}{|c|}{$\mathbf{H}$} & \multicolumn{2}{|c|}{$\mathbf{N}$} & \multicolumn{2}{|c|}{$\mathbf{O}$} \\
\hline & & Calc & Found & Calc & Found & Calc & Found & Calc & Found \\
\hline 5 & $\mathrm{C}_{26} \mathrm{H}_{45} \mathrm{~N}_{2} \mathrm{O}_{3}$ & 72.18 & 71.99 & 10.25 & 10.22 & 6.48 & 6.31 & 11.09 & 11.28 \\
\hline $7 \mathbf{a}$ & $\mathrm{C}_{28} \mathrm{H}_{46} \mathrm{~N}_{2} \mathrm{O}_{4} \cdot 0.5 \mathrm{H}_{2} \mathrm{O}$ & 69.53 & 69.51 & 9.79 & 9.61 & 5.79 & 5.39 & & \\
\hline $7 \mathbf{b}$ & $\mathrm{C}_{29} \mathrm{H}_{48} \mathrm{~N}_{2} \mathrm{O}_{4} \cdot 0.5 \mathrm{H}_{2} \mathrm{O}$ & 70.02 & 70.63 & 9.85 & 9.76 & 5.63 & 5.21 & & \\
\hline 7c & $\mathrm{C}_{31} \mathrm{H}_{52} \mathrm{~N}_{2} \mathrm{O}_{4} \cdot 0.5 \mathrm{H}_{2} \mathrm{O}$ & 70.85 & 71.29 & 10.09 & 9.93 & 5.33 & 5.04 & & \\
\hline 7d & $\mathrm{C}_{31} \mathrm{H}_{52} \mathrm{~N}_{2} \mathrm{O}_{4} \cdot 0.5 \mathrm{H}_{2} \mathrm{O}$ & 70.85 & 71.45 & 10.09 & 9.88 & 5.33 & 5.23 & & \\
\hline $7 e$ & $\mathrm{C}_{31} \mathrm{H}_{52} \mathrm{~N}_{2} \mathrm{O}_{4}$ & 72.05 & 71.88 & 10.14 & 10.34 & 5.42 & 5.42 & 12.38 & 11.87 \\
\hline 7f & $\mathrm{C}_{32} \mathrm{H}_{54} \mathrm{~N}_{2} \mathrm{O}_{4}$ & 72.41 & 72.14 & 10.25 & 10.18 & 5.28 & 5.23 & 12.06 & 11.99 \\
\hline $7 \mathbf{h}$ & $\mathrm{C}_{33} \mathrm{H}_{54} \mathrm{~N}_{2} \mathrm{O}_{4}$ & 73.02 & 72.76 & 10.03 & 9.94 & 5.16 & 5.07 & 11.79 & 11.68 \\
\hline $7 \mathbf{i}$ & $\mathrm{C}_{34} \mathrm{H}_{50} \mathrm{~N}_{2} \mathrm{O}_{4}$ & 74.14 & 73.98 & 9.15 & 9.12 & 5.09 & 4.97 & 11.62 & 11.61 \\
\hline $8 c$ & $\mathrm{C}_{31} \mathrm{H}_{52} \mathrm{~N}_{2} \mathrm{O}_{4}$ & 72.05 & 71.91 & 10.14 & 10.16 & 5.42 & 5.37 & 12.38 & 12.11 \\
\hline 8d & $\mathrm{C}_{31} \mathrm{H}_{52} \mathrm{~N}_{2} \mathrm{O}_{4}$ & 72.05 & 72.09 & 10.14 & 10.06 & 5.42 & 5.35 & 12.38 & 12.32 \\
\hline $8 \mathbf{8 h}$ & $\mathrm{C}_{33} \mathrm{H}_{54} \mathrm{~N}_{2} \mathrm{O}_{4} \cdot 0.5 \mathrm{H}_{2} \mathrm{O}$ & 71.83 & 72.13 & 10.05 & 9.91 & 5.08 & 4.92 & & \\
\hline $9 \mathbf{b}$ & $\mathrm{C}_{29} \mathrm{H}_{46} \mathrm{~N}_{2} \mathrm{O}_{3} \cdot 0.25 \mathrm{H}_{2} \mathrm{O}$ & 72.61 & 73.08 & 9.88 & 9.74 & 5.84 & 5.91 & & \\
\hline $9 \mathrm{~g}$ & $\mathrm{C}_{33} \mathrm{H}_{46} \mathrm{~N}_{2} \mathrm{O}_{3} \cdot \mathrm{H}_{2} \mathrm{O}$ & 73.84 & 73.25 & 9.01 & 9.56 & 5.22 & 5.98 & & \\
\hline $9 \mathrm{~h}$ & $\mathrm{C}_{33} \mathrm{H}_{52} \mathrm{~N}_{2} \mathrm{O}_{3}$ & 75.53 & 75.74 & 9.99 & 9.91 & 5.34 & 5.14 & & \\
\hline 10 & $\mathrm{C}_{30} \mathrm{H}_{50} \mathrm{~N}_{2} \mathrm{O}_{3} .0,5 \mathrm{H}_{2} \mathrm{O}$ & 72.68 & 73.38 & 10.37 & 10.54 & 5.65 & 5.63 & & \\
\hline 11 & $\mathrm{C}_{26} \mathrm{H}_{44} \mathrm{~N}_{2} \mathrm{O}_{3} \cdot 0.5 \mathrm{H}_{2} \mathrm{O}$ & 70.71 & 70.67 & 10.27 & 10.31 & 6.34 & 6.19 & 12.68 & 12.08 \\
\hline 13 & $\mathrm{C}_{30} \mathrm{H}_{50} \mathrm{~N}_{2} \mathrm{O}_{4} \cdot 0.5 \mathrm{H}_{2} \mathrm{O}$ & 70.41 & 70.84 & 10.05 & 9.71 & 5.47 & 5.21 & & \\
\hline 14 & $\mathrm{C}_{30} \mathrm{H}_{48} \mathrm{~N}_{2} \mathrm{O}_{3} \cdot 0.5 \mathrm{H}_{2} \mathrm{O}$ & 72.98 & 72.61 & 10.00 & 9.65 & 5.67 & 5.64 & & \\
\hline 18 & $\mathrm{C}_{29} \mathrm{H}_{48} \mathrm{~N}_{2} \mathrm{O}_{4}$ & 71.27 & 70.26 & 9.90 & 9.58 & 5.73 & 5.44 & 13.10 & 12.75 \\
\hline 20a & $\mathrm{C}_{32} \mathrm{H}_{52} \mathrm{~N}_{2} \mathrm{O}_{5}$ & 70.55 & 70.64 & 9.62 & 9.84 & 5.14 & 4.89 & 14.68 & 14.48 \\
\hline $20 b$ & $\mathrm{C}_{33} \mathrm{H}_{55} \mathrm{~N}_{2} \mathrm{O}_{5}$ & 70.93 & 70.82 & 9.74 & 10.05 & 5.01 & 4.95 & 14.32 & 14.16 \\
\hline $24 a$ & $\mathrm{C}_{37} \mathrm{H}_{57} \mathrm{~N}_{3} \mathrm{O}_{3} \cdot 0.5 \mathrm{H}_{2} \mathrm{O}$ & 73.96 & 74.14 & 9.73 & 9.63 & 6.99 & 7.13 & 9.32 & 9.11 \\
\hline $26 \mathbf{a}$ & $\mathrm{C}_{30} \mathrm{H}_{49} \mathrm{~N}_{3} \mathrm{O}_{2} \cdot 0.5 \mathrm{H}_{2} \mathrm{O}$ & 73.13 & 72.02 & 10.23 & 10.01 & 8.53 & 8.11 & & \\
\hline 27 & $\mathrm{C}_{33} \mathrm{H}_{54} \mathrm{~N}_{2} \mathrm{O}_{5}$ & 70.93 & 70.82 & 9.74 & 10.05 & 5.01 & 4.95 & 14.32 & 14.16 \\
\hline 28 & $\mathrm{C}_{33} \mathrm{H}_{52} \mathrm{~N}_{2} \mathrm{O}_{5} \cdot 0.5 \mathrm{H}_{2} \mathrm{O}$ & 70.05 & 70.06 & 9.61 & 9.61 & 4.95 & 4.55 & & \\
\hline & & & & & & & & & \\
\hline
\end{tabular}




\section{Crystal data and structure refinement for compound 1.}

Table 5. Crystal data and structure refinement for compound $\mathbf{1 .}$

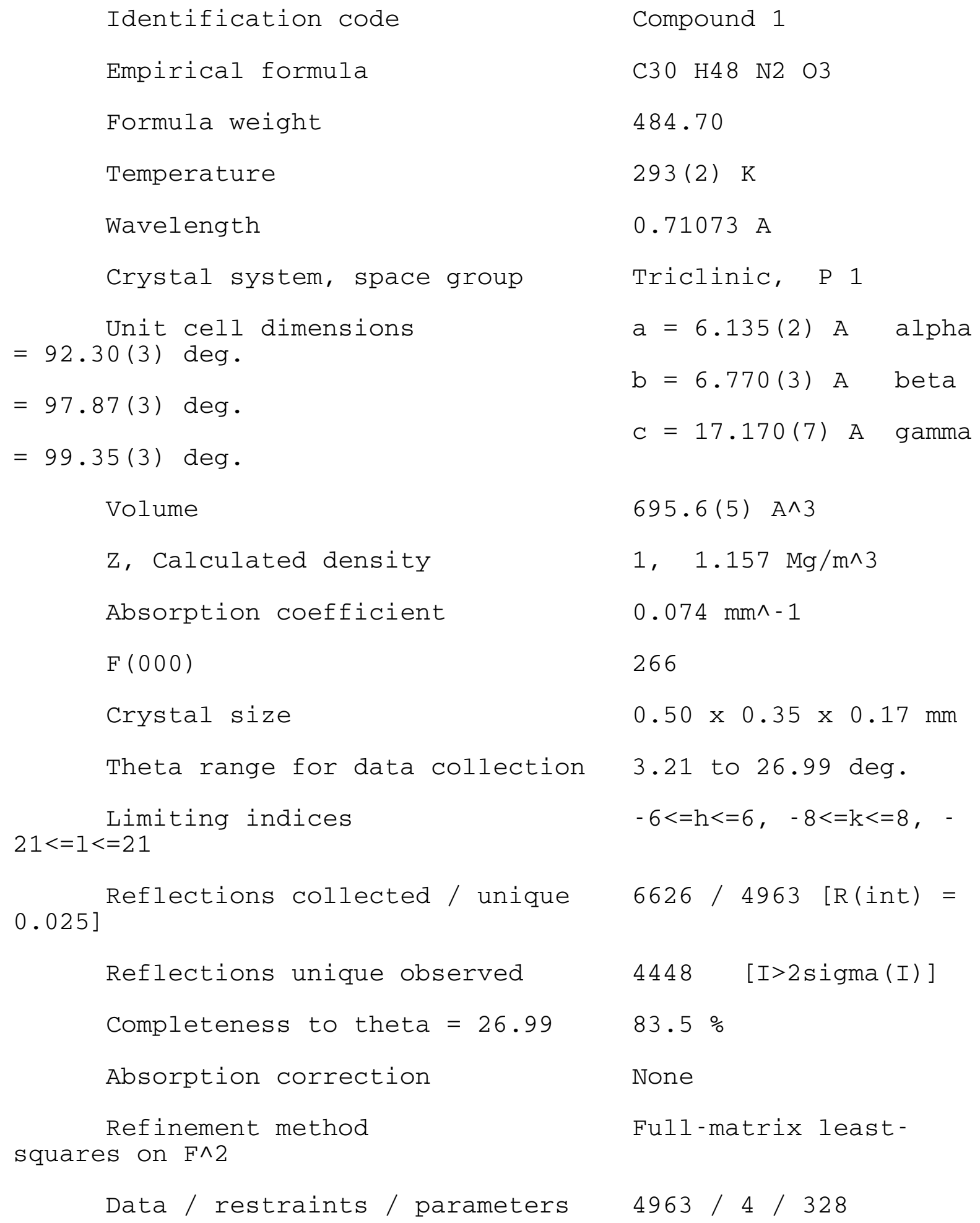




\begin{tabular}{|c|c|}
\hline Goodness-of-fit on $F^{\wedge} 2$ & 1.024 \\
\hline $\begin{array}{l}\text { Final } R \text { indices }[I>2 \text { sigma (I) }] \\
\left.0.1343^{(4448} \text { Fo }\right)\end{array}$ & $\mathrm{R} 1=0.0501, \mathrm{wR} 2=$ \\
\hline $\begin{array}{l}0.1419^{\mathrm{R}} \text { (4ndices (all data) } \\
03 \text { data) }\end{array}$ & $\mathrm{R} 1=0.0570, \mathrm{wR} 2=$ \\
\hline Extinction coefficient & $0.07(2)$ \\
\hline Largest diff. peak and hole & 0.203 and -0.179 e.A^-3 \\
\hline
\end{tabular}


Table 6. Atomic coordinates ( $\times 10^{\wedge} 4$ ) and equivalent isotropic displacement parameters $\left(\mathrm{A}^{\wedge} 2 \times 10^{\wedge} 3\right)$ for 1.

orthogonalized

$\mathrm{U}(\mathrm{eq})$ is defined as one third of the trace of the

Uij tensor.

\begin{tabular}{|c|c|c|c|c|}
\hline \multicolumn{2}{|l|}{$\begin{array}{l}- \\
\mathrm{U}(\mathrm{eq})\end{array}$} & $\mathrm{x}$ & Y & z \\
\hline \multicolumn{5}{|l|}{-} \\
\hline & C (1) & $-131(5)$ & $3117(4)$ & $-1911(2)$ \\
\hline & $C(2)$ & $290(5)$ & $4055(5)$ & $-2667(2)$ \\
\hline & $C(3)$ & $1196(4)$ & $6272(4)$ & $-2527(1)$ \\
\hline $44(1)$ & $C(4)$ & $3023(4)$ & $6697(4)$ & $-1803(1)$ \\
\hline $40(1)$ & $C(5)$ & $1902(4)$ & $6104(3)$ & - 1074 (1) \\
\hline $46(1)$ & C (6) & $3529(4)$ & $6514(4)$ & $-295(1)$ \\
\hline $44(1)$ & $C(7)$ & $2440(4)$ & $6984(3)$ & $422(1)$ \\
\hline $37(1)$ & C ( 8) & $1251(4)$ & $5163(3)$ & 787 (1) \\
\hline $39(1)$ & C (9) & $-646(4)$ & $3894(3)$ & 201 (1) \\
\hline $44(1)$ & $C(10)$ & $610(4)$ & $3971(3)$ & $-1188(1)$ \\
\hline $52(1)$ & C (11) & -2301 ( 5 ) & $2403(4)$ & $584(2)$ \\
\hline $47(1)$ & C (12) & $-2593(4)$ & $2698(4)$ & $1440(1)$ \\
\hline $38(1)$ & $C(13)$ & $-475(4)$ & $3771(3)$ & $1958(1)$ \\
\hline $36(1)$ & C (14) & 429 (3) & $5713(3)$ & $1561(1)$ \\
\hline $44(1)$ & C (15) & $2256(4)$ & $6796(3)$ & $2213(1)$ \\
\hline $50(1)$ & $C(16)$ & $1389(4)$ & $6263(4)$ & 2989 (1) \\
\hline $43(1)$ & C (17) & $-708(4)$ & $4619(3)$ & $2788(1)$ \\
\hline $49(1)$ & C (18) & $1234(4)$ & $2319(3)$ & $2040(2)$ \\
\hline $48(1)$ & C (19) & $210(4)$ & $2808(3)$ & $-476(1)$ \\
\hline $53(1)$ & $C(20)$ & $-925(5)$ & $3213(4)$ & $3464(1)$ \\
\hline
\end{tabular}




\begin{tabular}{|c|c|c|c|c|}
\hline & $\mathrm{C}(21)$ & $-2929(6)$ & $1492(5)$ & $3307(2)$ \\
\hline 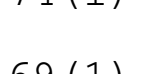 & NB & $-870(5)$ & $4478(4)$ & $4196(1)$ \\
\hline$(1)$ & $C(22)$ & $-2954(9)$ & $5130(8)$ & $4280(3)$ \\
\hline & $C(23)$ & $-24(9)$ & $3515(7)$ & $4893(2)$ \\
\hline & $O(11)$ & $-3495(5)$ & $1066(4)$ & $185(1)$ \\
\hline $78(1)$ & $O(16)$ & $943(5)$ & $8033(3)$ & $3377(1)$ \\
\hline $49(1)$ & $\mathrm{C}(28)$ & $-1360(4)$ & $7073(3)$ & $1398(1)$ \\
\hline 1 (1) & C (29) & $3788(5)$ & $8962(4)$ & $-1774(2)$ \\
\hline $62(1)$ & $C(30)$ & $4951(5)$ & $5589(5)$ & $-1877(2)$ \\
\hline $65(1)$ & NA & $1979(4)$ & $7073(4)$ & $-3247(1)$ \\
\hline $64(1)$ & $C\left(1^{\prime}\right)$ & $3522(5)$ & $8574(5)$ & $-3166(2)$ \\
\hline $79(1)$ & $O\left(1^{\prime}\right)$ & $4556(4)$ & $9597(3)$ & $-2492(1)$ \\
\hline $77(1)$ & $C\left(2^{\prime}\right)$ & $4579(7)$ & $9473(6)$ & $-3855(2)$ \\
\hline $104(1)$ & $C\left(3^{\prime}\right)$ & $6875(8)$ & $8971(8)$ & $-3820(3)$ \\
\hline $141(2)$ & $C\left(4^{\prime}\right)$ & $3173(11)$ & 8811 ( 12 ) & $-4629(3)$ \\
\hline
\end{tabular}


Table 7. Hydrogen coordinates ( $\left.\mathrm{x} 10^{\wedge} 4\right)$ and isotropic displacement parameters $\left(\mathrm{A}^{\wedge} 2 \mathrm{x}\right.$ $10^{\wedge} 3$ ) for 1.

\begin{tabular}{|c|c|c|c|c|}
\hline $\mathrm{U}(\mathrm{eq})$ & & $\mathrm{x}$ & $\mathrm{Y}$ & $\mathrm{z}$ \\
\hline & $\mathrm{H}(1)$ & -978 & 1836 & -1950 \\
\hline 0 & $\mathrm{H}(2 \mathrm{~A})$ & -1093 & 3861 & -3032 \\
\hline & $\mathrm{H}(2 \mathrm{~B})$ & 1350 & 3396 & -2905 \\
\hline 10 & $\mathrm{H}(3)$ & -38 & 6946 & -2417 \\
\hline 0 & $\mathrm{H}(5)$ & 790 & 6980 & -1040 \\
\hline & $H(6 A)$ & 4239 & 5349 & -201 \\
\hline & $\mathrm{H}(6 \mathrm{~B})$ & 4689 & 7635 & -352 \\
\hline 50 & $H(7 A)$ & 1366 & 7855 & 270 \\
\hline 50 & $\mathrm{H}(7 \mathrm{~B})$ & 3582 & 7725 & 822 \\
\hline 11 & $\mathrm{H}(8)$ & 2368 & 4301 & 922 \\
\hline 44 & $\mathrm{H}(9)$ & -1521 & 4848 & -48 \\
\hline 53 & $\mathrm{H}(12 \mathrm{~A})$ & -3046 & 1398 & 1640 \\
\hline 53 & $\mathrm{H}(12 \mathrm{~B})$ & -3781 & 3471 & 1474 \\
\hline 50 & $\mathrm{H}(15 \mathrm{~A})$ & 3660 & 6331 & 2186 \\
\hline 50 & $\mathrm{H}(15 \mathrm{~B})$ & 2476 & 8234 & 2163 \\
\hline 55 & $\mathrm{H}(16)$ & 2537 & 5723 & 3330 \\
\hline 48 & $\mathrm{H}(17)$ & -2014 & 5292 & 2733 \\
\hline 56 & $\mathrm{H}(18 \mathrm{~A})$ & 754 & 1267 & 2368 \\
\hline 56 & $\mathrm{H}(18 \mathrm{~B})$ & 2669 & 3040 & 2273 \\
\hline 56 & $\mathrm{H}(18 \mathrm{C})$ & 1342 & 1747 & 1528 \\
\hline 53 & $\mathrm{H}(19 \mathrm{~A})$ & 1598 & 2390 & -264 \\
\hline 53 & $\mathrm{H}(19 \mathrm{~B})$ & -862 & 1602 & -651 \\
\hline 59 & $\mathrm{H}(20)$ & 422 & 2596 & 3532 \\
\hline
\end{tabular}




\begin{tabular}{|c|c|c|c|c|}
\hline & $\mathrm{H}(21 \mathrm{~A})$ & -2689 & 561 & 2903 \\
\hline & $\mathrm{H}(21 \mathrm{~B})$ & -4261 & 2025 & 3137 \\
\hline & $\mathrm{H}(21 \mathrm{C})$ & -3090 & 815 & 3782 \\
\hline & $\mathrm{H}(22 \mathrm{~A})$ & -3383 & 5884 & 3839 \\
\hline & $\mathrm{H}(22 \mathrm{~B})$ & -2779 & 5960 & 4758 \\
\hline & $\mathrm{H}(22 \mathrm{C})$ & -4088 & 3982 & 4297 \\
\hline & $\mathrm{H}(23 \mathrm{~A})$ & 1383 & 3135 & 4827 \\
\hline 22 & $\mathrm{H}(23 \mathrm{~B})$ & -1072 & 2342 & 4964 \\
\hline 122 & $\mathrm{H}(23 \mathrm{C})$ & 172 & 4434 & 5348 \\
\hline 90 & HO16 & $460(70)$ & $7530(60)$ & $3753(18)$ \\
\hline 6 & $\mathrm{H}(28 \mathrm{~A})$ & -1816 & 7468 & 1886 \\
\hline 0 & $\mathrm{H}(28 \mathrm{~B})$ & -2628 & 6350 & 1054 \\
\hline 56 & $\mathrm{H}(28 \mathrm{C})$ & -745 & 8246 & 1154 \\
\hline 69 & $\mathrm{H}(29 \mathrm{~A})$ & 2557 & 9632 & -1681 \\
\hline 69 & $\mathrm{H}(29 \mathrm{~B})$ & 4985 & 9355 & -1338 \\
\hline 71 & $\mathrm{H}(30 \mathrm{~A})$ & 4471 & 4181 & -1833 \\
\hline 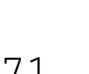 & $\mathrm{H}(30 \mathrm{~B})$ & 6162 & 6085 & -1464 \\
\hline 71 & $\mathrm{H}(30 \mathrm{C})$ & 5440 & 5799 & -2379 \\
\hline 86 & $\mathrm{H}\left(2^{\prime}\right.$ ) & 4726 & 10935 & -3786 \\
\hline 120 & $\mathrm{H}\left(\mathrm{B}^{\prime} \mathrm{A}\right)$ & 6785 & 7540 & -3849 \\
\hline 120 & $\mathrm{H}\left(\mathrm{3}^{\prime} \mathrm{B}\right)$ & 7765 & 9531 & -3334 \\
\hline 120 & $\mathrm{H}\left(\mathrm{J}^{\prime} \mathrm{C}\right)$ & 7548 & 9519 & -4255 \\
\hline- & $\mathrm{H}\left(4{ }^{\prime} \mathrm{A}\right)$ & 1798 & 9327 & -4655 \\
\hline 6 & $\mathrm{H}\left(4{ }^{\prime} \mathrm{B}\right)$ & 2857 & 7373 & -4684 \\
\hline & $\mathrm{H}\left(4^{\prime} \mathrm{C}\right)$ & 3959 & 9309 & -5047 \\
\hline
\end{tabular}


Table 8. Bond lengths [A] and angles [deg] for $\mathbf{1}$.

\begin{tabular}{|c|c|}
\hline$C(1)-C(10)$ & $1.336(3)$ \\
\hline$C(1)-C(2)$ & $1.504(4)$ \\
\hline$C(2)-C(3)$ & $1.511(4)$ \\
\hline$C(3)-N A$ & $1.479(3)$ \\
\hline$C(3)-C(4)$ & $1.538(3)$ \\
\hline$C(4)-C(30)$ & $1.516(4)$ \\
\hline$C(4)-C(29)$ & $1.525(4)$ \\
\hline$C(4)-C(5)$ & $1.545(3)$ \\
\hline$C(5)-C(10)$ & $1.522(3)$ \\
\hline$C(5)-C(6)$ & $1.541(3)$ \\
\hline$C(6)-C(7)$ & $1.525(3)$ \\
\hline$C(7)-C(8)$ & $1.530(3)$ \\
\hline$C(8)-C(14)$ & $1.538(3)$ \\
\hline$C(8)-C(9)$ & $1.546(3)$ \\
\hline$C(9)-C(11)$ & $1.544(3)$ \\
\hline$C(9)-C(19)$ & $1.547(3)$ \\
\hline$C(10)-C(19)$ & $1.506(3)$ \\
\hline$C(11)-O(11)$ & $1.193(3)$ \\
\hline$C(11)-C(12)$ & $1.514(3)$ \\
\hline$C(12)-C(13)$ & $1.527(3)$ \\
\hline$C(13)-C(18)$ & $1.547(3)$ \\
\hline$C(13)-C(17)$ & $1.550(3)$ \\
\hline$C(13)-C(14)$ & $1.561(3)$ \\
\hline$C(14)-C(15)$ & $1.539(3)$ \\
\hline$C(14)-C(28)$ & $1.548(3)$ \\
\hline$C(15)-C(16)$ & $1.536(3)$ \\
\hline$C(16)-O(16)$ & $1.430(3)$ \\
\hline$C(16)-C(17)$ & $1.547(4)$ \\
\hline$C(17)-C(20)$ & $1.536(3)$ \\
\hline$C(20)-N B$ & $1.486(4)$ \\
\hline$C(20)-C(21)$ & $1.537(4)$ \\
\hline NB - C (22) & $1.442(5)$ \\
\hline NB - C (23) & $1.459(5)$ \\
\hline$C(29)-O\left(1^{\prime}\right)$ & $1.436(3)$ \\
\hline $\mathrm{NA}-\mathrm{C}\left(1^{\prime}\right)$ & $1.260(4)$ \\
\hline$C\left(1^{\prime}\right)-O\left(1^{\prime}\right)$ & $1.346(4)$ \\
\hline$C\left(1^{\prime}\right)-C\left(2^{\prime}\right)$ & $1.526(4)$ \\
\hline$C\left(2^{\prime}\right)-C\left(4^{\prime}\right)$ & $1.492(6)$ \\
\hline$C\left(2^{\prime}\right)-C\left(3^{\prime}\right)$ & $1.495(6)$ \\
\hline$C(10)-C(1)-C(2)$ & $125.4(2)$ \\
\hline$C(1)-C(2)-C(3)$ & $111.3(2)$ \\
\hline$N A-C(3)-C(2)$ & $110.0(2)$ \\
\hline$N A-C(3)-C(4)$ & $112.4(2)$ \\
\hline$C(2)-C(3)-C(4)$ & $111.4(2)$ \\
\hline$C(30)-C(4)-C(29)$ & $111.0(2)$ \\
\hline$C(30)-C(4)-C(3)$ & $112.5(2)$ \\
\hline$C(29)-C(4)-C(3)$ & $103.9(2)$ \\
\hline$C(30)-C(4)-C(5)$ & $111.4(2)$ \\
\hline$C(29)-C(4)-C(5)$ & $110.5(2)$ \\
\hline$C(3)-C(4)-C(5)$ & $107.2(2)$ \\
\hline$C(10)-C(5)-C(6)$ & $113.4(2)$ \\
\hline$C(10)-C(5)-C(4)$ & $111.4(2)$ \\
\hline$C(6)-C(5)-C(4)$ & $113.1(2)$ \\
\hline
\end{tabular}




\begin{tabular}{|c|c|}
\hline$C(7)-C(6)-C(5)$ & $114.4(2)$ \\
\hline$C(6)-C(7)-C(8)$ & $115.5(2)$ \\
\hline$C(7)-C(8)-C(14)$ & $113.0(2)$ \\
\hline$C(7)-C(8)-C(9)$ & $112.7(2)$ \\
\hline$C(14)-C(8)-C(9)$ & $111.4(2)$ \\
\hline$C(11)-C(9)-C(8)$ & $114.8(2)$ \\
\hline$C(11)-C(9)-C(19)$ & $110.3(2)$ \\
\hline$C(8)-C(9)-C(19)$ & $113.2(2)$ \\
\hline$C(1)-C(10)-C(19)$ & $120.1(2)$ \\
\hline$C(1)-C(10)-C(5)$ & $120.5(2)$ \\
\hline$C(19)-C(10)-C(5)$ & $119.4(2)$ \\
\hline $\mathrm{O}(11)-\mathrm{C}(11)-\mathrm{C}(12)$ & $118.8(2)$ \\
\hline $\mathrm{O}(11)-\mathrm{C}(11)-\mathrm{C}(9)$ & $119.4(2)$ \\
\hline$C(12)-C(11)-C(9)$ & $121.6(2)$ \\
\hline$C(11)-C(12)-C(13)$ & $113.1(2)$ \\
\hline$C(12)-C(13)-C(18)$ & $108.2(2)$ \\
\hline$C(12)-C(13)-C(17)$ & $117.6(2)$ \\
\hline$C(18)-C(13)-C(17)$ & $109.1(2)$ \\
\hline$C(12)-C(13)-C(14)$ & $108.4(2)$ \\
\hline$C(18)-C(13)-C(14)$ & $111.6(2)$ \\
\hline$C(17)-C(13)-C(14)$ & $101.9(2)$ \\
\hline$C(8)-C(14)-C(15)$ & $115.1(2)$ \\
\hline$C(8)-C(14)-C(28)$ & $109.7(2)$ \\
\hline$C(15)-C(14)-C(28)$ & $107.8(2)$ \\
\hline$C(8)-C(14)-C(13)$ & $110.2(2)$ \\
\hline$C(15)-C(14)-C(13)$ & $102.0(2)$ \\
\hline$C(28)-C(14)-C(13)$ & $111.9(2)$ \\
\hline$C(16)-C(15)-C(14)$ & $105.0(2)$ \\
\hline$O(16)-C(16)-C(15)$ & $109.5(2)$ \\
\hline$O(16)-C(16)-C(17)$ & $112.8(2)$ \\
\hline$C(15)-C(16)-C(17)$ & $107.6(2)$ \\
\hline$C(20)-C(17)-C(16)$ & $110.8(2)$ \\
\hline$C(20)-C(17)-C(13)$ & $120.1(2)$ \\
\hline$C(16)-C(17)-C(13)$ & $102.9(2)$ \\
\hline$C(10)-C(19)-C(9)$ & $117.1(2)$ \\
\hline$N B-C(20)-C(17)$ & $107.5(2)$ \\
\hline$N B-C(20)-C(21)$ & $113.8(2)$ \\
\hline$C(17)-C(20)-C(21)$ & $114.5(2)$ \\
\hline$C(22)-N B-C(23)$ & $111.5(3)$ \\
\hline$C(22)-N B-C(20)$ & $114.3(3)$ \\
\hline$C(23)-N B-C(20)$ & $111.4(3)$ \\
\hline$O\left(1^{\prime}\right)-C(29)-C(4)$ & $112.0(2)$ \\
\hline$C\left(1^{\prime}\right)-N A-C(3)$ & $117.8(2)$ \\
\hline$N A-C\left(1^{\prime}\right)-O\left(1^{\prime}\right)$ & $127.9(3)$ \\
\hline $\mathrm{NA}-\mathrm{C}\left(1^{\prime}\right)-\mathrm{C}\left(2^{\prime}\right)$ & $123.2(3)$ \\
\hline $\mathrm{O}\left(1^{\prime}\right)-\mathrm{C}\left(1^{\prime}\right)-\mathrm{C}\left(2^{\prime}\right)$ & $108.9(3)$ \\
\hline$C\left(1^{\prime}\right)-O\left(1^{\prime}\right)-C(29)$ & $117.1(2)$ \\
\hline$C\left(4^{\prime}\right)-C\left(2^{\prime}\right)-C\left(3^{\prime}\right)$ & $112.1(4)$ \\
\hline$C\left(4^{\prime}\right)-C\left(2^{\prime}\right)-C\left(1^{\prime}\right)$ & $112.3(3)$ \\
\hline$C\left(3^{\prime}\right)-C\left(2^{\prime}\right)-C\left(1^{\prime}\right)$ & 108.913 \\
\hline
\end{tabular}


Table 9. Selected torsion angles [deg] for $\mathbf{1}$.

\begin{tabular}{|c|c|}
\hline & $C(10)-C(1)-C(2)-C(3)$ \\
\hline $4(3)$ & $C(1)-C(2)-C(3)-C(4)$ \\
\hline $5.1(3)$ & $C(2)-C(3)-C(4)-C(5)$ \\
\hline $2.8(3)$ & $\begin{array}{l}C(3)-C(4)-C(5)-C(10) \\
C(10)-C(5)-C(6)-C(7)\end{array}$ \\
\hline $1.6(2)$ & $C(5)-C(6)-C(7)-C(8)$ \\
\hline $9.4(3)$ & $C(6)-C(7)-C(8)-C(9)$ \\
\hline $5.9(3)$ & $C(14)-C(8)-C(9)-C(11)$ \\
\hline $58.1(2)$ & $C(2)-C(1)-C(10)-C(5)$ \\
\hline $.2(4)$ & $C(4)-C(5)-C(10)-C(1)$ \\
\hline $6.0(3)$ & $C(6)-C(5)-C(10)-C(19)$ \\
\hline $33.9(4)$ & $C(19)-C(9)-C(11)-O(11)$ \\
\hline $22.3(3)$ & $C(8)-C(9)-C(11)-C(12)$ \\
\hline $30.2(3)$ & $C(9)-C(11)-C(12)-C(13)$ \\
\hline $50.5(3)$ & $C(11)-C(12)-C(13)-C(14)$ \\
\hline $57.8(2)$ & $C(7)-C(8)-C(14)-C(15)$ \\
\hline $64.2(2)$ & $C(9)-C(8)-C(14)-C(28)$ \\
\hline $59.5(2)$ & $C(9)-C(8)-C(14)-C(13)$ \\
\hline $67.3(2)$ & $C(12)-C(13)-C(14)-C(8)$ \\
\hline $45.3(2)$ & $C(17)-C(13)-C(14)-C(15)$ \\
\hline $55.1(2)$ & $C(12)-C(13)-C(14)-C(28)$ \\
\hline $33.4(2)$ & $C(13)-C(14)-C(15)-C(16)$ \\
\hline $113.8(2)$ & $C(14)-C(15)-C(16)-O(16)$ \\
\hline $9.2(2)$ & $C(14)-C(15)-C(16)-C(17)$ \\
\hline $.6(2)$ & $O(16)-C(16)-C(17)-C(20)$ \\
\hline
\end{tabular}




$$
\begin{aligned}
& 18.9(2) \\
& C(15)-C(16)-C(17)-C(13) \\
& 78.7(3) \\
& C(12)-C(13)-C(17)-C(20) \\
& 47.2(3) \\
& C(5)-C(10)-C(19)-C(9) \\
& 85.8(2) \\
& C(8)-C(9)-C(19)-C(10) \\
& 54.2(3) \\
& \mathrm{C}(16)-\mathrm{C}(17)-\mathrm{C}(20)-\mathrm{NB} \\
& 58.5(3) \\
& C(13)-C(17)-C(20)-C(21) \\
& 79.3(3) \\
& \mathrm{C}(17)-\mathrm{C}(20)-\mathrm{NB}-\mathrm{C}(22) \\
& 48.6(4) \\
& \mathrm{C}(21)-\mathrm{C}(20)-\mathrm{NB}-\mathrm{C}(22) \\
& 153.1(3) \\
& C(17)-C(20)-N B-C(23) \\
& \text { C (21) - C (20)-NB - C (23) } \\
& 78.9(4) \\
& \mathrm{NA}-\mathrm{C}(3)-\mathrm{C}(4)-\mathrm{C}(29) \\
& 53.9(3) \\
& C(3)-C(4)-C(29)-O\left(1^{\prime}\right) \\
& 57.3(3) \\
& 34.0(4) \\
& C(4)-C(29)-O\left(1^{\prime}\right)-C\left(1^{\prime}\right) \\
& 17.3(6) \\
& 164.7(4) \\
& N A-C\left(1^{\prime}\right)-C\left(2^{\prime}\right)-C\left(4^{\prime}\right) \\
& O\left(1^{\prime}\right)-C\left(1^{\prime}\right)-C\left(2^{\prime}\right)-C\left(4^{\prime}\right) \\
& 107.5(4) \\
& \mathrm{NA}-\mathrm{C}\left(1^{\prime}\right)-\mathrm{C}\left(2^{\prime}\right)-\mathrm{C}\left(3^{\prime}\right) \\
& 70.5(4) \\
& O\left(1^{\prime}\right)-C\left(1^{\prime}\right)-C\left(2^{\prime}\right)-C\left(3^{\prime}\right)
\end{aligned}
$$


Table 10. Anisotropic displacement parameters $\left(A^{\wedge} 2 \times 10^{\wedge} 3\right)$ for 1. takes the form:

The anisotropic displacement factor exponent U12] $-2 \mathrm{pi} \wedge 2[\mathrm{~h} \wedge 2 \mathrm{a} * \wedge 2 \mathrm{U} 11+\ldots+2 \mathrm{~h} k \mathrm{k} * \mathrm{~b} *$

$\mathrm{U} 12$

$\mathrm{U} 11$

$\mathrm{U} 22$

U33

U23

U13

\begin{tabular}{|c|c|c|c|c|c|}
\hline$-9(1)^{C(1)}$ & $57(2)$ & $62(2)$ & $50(1)$ & $-16(1)$ & $9(1)$ \\
\hline $\begin{array}{c}C(2) \\
-16(1)\end{array}$ & $65(2)$ & $83(2)$ & $45(1)$ & $-15(1)$ & $6(1)$ \\
\hline $3(1)^{\mathrm{C}(3)}$ & $42(2)$ & $78(2)$ & $38(1)$ & $-2(1)$ & $3(1)$ \\
\hline $0(1)^{\mathrm{C}(4)}$ & $38(1)$ & $54(1)$ & $38(1)$ & $-1(1)$ & $5(1)$ \\
\hline $0(1)^{C(5)}$ & $34(1)$ & $44(1)$ & $38(1)$ & $-3(1)$ & $3(1)$ \\
\hline$-8(1)^{C(6)}$ & $40(1)$ & $50(1)$ & $41(1)$ & $0(1)$ & $0(1)$ \\
\hline $\begin{array}{c}C(7) \\
-10(1)\end{array}$ & $48(1)$ & $36(1)$ & $41(1)$ & $-4(1)$ & $1(1)$ \\
\hline $1(1)^{C(8)}$ & $34(1)$ & $32(1)$ & $41(1)$ & $0(1)$ & $0(1)$ \\
\hline$-1(1)^{C(9)}$ & $36(1)$ & $35(1)$ & $43(1)$ & $-2(1)$ & $0(1)$ \\
\hline $0(1)^{C(10)}$ & $36(1)$ & $45(1)$ & $47(1)$ & $-8(1)$ & $5(1)$ \\
\hline $\begin{array}{c}C(11) \\
-13(1)\end{array}$ & $53(2)$ & 49 (1) & $47(1)$ & $0(1)$ & $5(1)$ \\
\hline$-7(1)^{C(12)}$ & $40(1)$ & $45(1)$ & $50(1)$ & $2(1)$ & $5(1)$ \\
\hline $3(1)^{C(13)}$ & $36(1)$ & $34(1)$ & $44(1)$ & $3(1)$ & $5(1)$ \\
\hline $3(1)^{C(14)}$ & $36(1)$ & $30(1)$ & $41(1)$ & $0(1)$ & $3(1)$ \\
\hline$-2(1)^{C(15)}$ & $47(1)$ & $36(1)$ & $44(1)$ & $-1(1)$ & $-1(1)$ \\
\hline $2(1)^{C(16)}$ & $55(2)$ & $50(1)$ & $39(1)$ & $1(1)$ & $0(1)$ \\
\hline $12(1)^{C(17)}$ & $44(1)$ & $45(1)$ & $42(1)$ & $6(1)$ & $5(1)$ \\
\hline $13(1)^{\mathrm{C}(18)}$ & $54(2)$ & $39(1)$ & $56(1)$ & $10(1)$ & $12(1)$ \\
\hline $0(1)^{\mathrm{C}(19)}$ & $52(1)$ & $35(1)$ & $51(1)$ & $-8(1)$ & $6(1)$ \\
\hline $10(1)^{C(20)}$ & $51(2)$ & $64(2)$ & $45(1)$ & $13(1)$ & $10(1)$ \\
\hline$-5(2)^{C(21)}$ & $74(2)$ & $78(2)$ & $65(2)$ & $23(2)$ & $15(2)$ \\
\hline
\end{tabular}




\begin{tabular}{|c|c|c|c|c|c|}
\hline $11(1)^{\mathrm{NB}}$ & $81(2)$ & $85(2)$ & $44(1)$ & $11(1)$ & $18(1)$ \\
\hline $41(3)^{C(22)}$ & $127(4)$ & $122(3)$ & $88(3)$ & $2(2)$ & $54(3)$ \\
\hline$-4(3)^{C(23)}$ & $132(4)$ & $126(3)$ & $49(2)$ & $26(2)$ & $2(2)$ \\
\hline $\begin{array}{c}O(11) \\
-84(2)\end{array}$ & $135(2)$ & $108(2)$ & $59(1)$ & $-22(1)$ & $21(1)$ \\
\hline $0(1)^{O(16)}$ & $123(2)$ & $57(1)$ & $51(1)$ & -11 (1) & $24(1)$ \\
\hline $18(1)^{C(28)}$ & $58(2)$ & $42(1)$ & $49(1)$ & $0(1)$ & $3(1)$ \\
\hline$-4(1)^{C(29)}$ & $75(2)$ & $61(2)$ & $45(1)$ & $5(1)$ & $14(1)$ \\
\hline $9(1)^{C(30)}$ & $43(2)$ & $84(2)$ & $59(2)$ & $4(1)$ & $12(1)$ \\
\hline $5(1)^{\mathrm{NA}}$ & $59(2)$ & $96(2)$ & $38(1)$ & $6(1)$ & $6(1)$ \\
\hline $7(1)^{C\left(1^{\prime}\right)}$ & $63(2)$ & $83(2)$ & $45(1)$ & $8(1)$ & $10(1)$ \\
\hline $\begin{array}{c}O\left(1^{\prime}\right) \\
-17(1)\end{array}$ & $100(2)$ & $78(1)$ & $49(1)$ & $7(1)$ & $15(1)$ \\
\hline $5(2)^{C\left(2^{\prime}\right)}$ & $88(3)$ & $91(2)$ & $53(2)$ & $13(2)$ & $20(2)$ \\
\hline $6(3)^{C(3 ')}$ & $101(3)$ & $136(4)$ & 79 (2) & $17(2)$ & $37(2)$ \\
\hline $\begin{array}{l}C(4 ') \\
-14(4)\end{array}$ & $124(4)$ & $221(7)$ & $62(2)$ & $32(3)$ & $7(2)$ \\
\hline
\end{tabular}

